

Digitized by the Internet Archive in 2007 with funding from Microsoft Corporation 






\section{H a n d b o o k - of- Rectal Diseases}






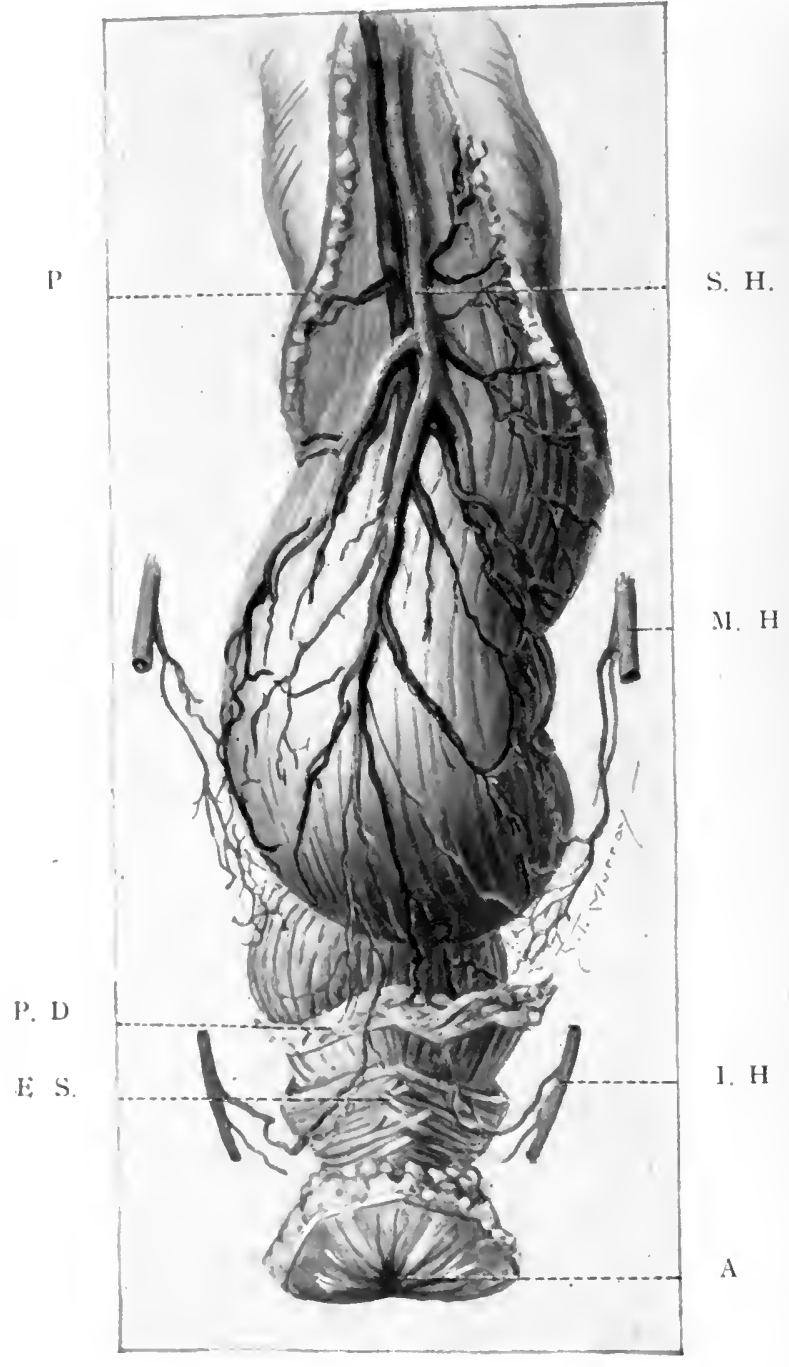

PI.ATE I.

THE BLOOD VESSELS OF TIIE RECTU.M.

S. H. Superior haemorrhoidal artery. M. H. Niddle haemorrhoidal artery. L. H. Inferior haemorrhoidal artery. A. Anus. E.S. External sphincter muscle, P. D. Pelvic diaphragm. P. Cut edge of peritoneum.

The Rectum: Its Diseases and Developmental Defects, by Sir Cliaries Ball. 


\section{Hand Book}

OF

\section{Diseases of the Rectum}

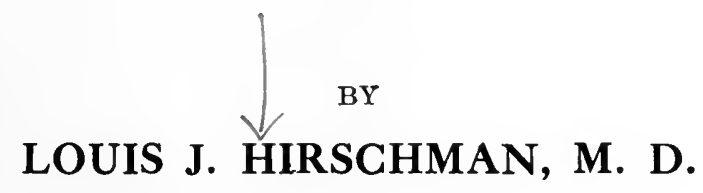

DETROIT, MICHIGAN, U. S. A.

FELLOW AMERICAN PROCTOLOGIC SOCIETY; LECTURER ON RECTAL SURGERY AND CLINICAL PROFESSOR OF PROCTOLOGY, DETROIT COLLEGE OF MEDICINE; ATTENDING PROCTOLOGIST, HARPER HOSPITAL: CONSULTING GYNECOLOGIST, DETROIT GERMAN POLYCLINIC; COLLABORATOR ON PROCTOLOGY, "P HYSICIAN AND SURGEON"; EDITOR "HARPLR HOSPITAL BULLETIN"; CHAIRMAN SECTION ON SURGERY, MICHIGAN S'TA'T'E MEDICAL SOCI. ETY; EX-PRESIDENT AIUMNI ASSOCIA'TION, DETROIT COJ,LEGE OF MEDICINE, ETC., HTC.

WITH ONE HUNDRED AND FORTY-SEVEN ILLUSTRATIONS, MOSTLY ORIGINAL, INCLUDING TWO COLORED PLATES.

St. Louis, C. V. MOSBY MEdICAL BOOK \& PUBLISHING CO. 
7917.7911909

$7: 9197 \%, 7 ! 5071.1(19$

$17, ?+\cdots,-11: 4: 2\}: 4$

Copyrighted by C. V. Mosby Co., 1909. 


\section{TO THE \\ MEMORY OF MY FATHER, \\ FREDERICK LOUIS HIRSCHMAN, M. D.,}

A country doctor whose untimely death ivas a sacrifice to the duties of that ozerworked and wholly unapprciatcd class of our profession-the Country Doctor: this work is affectionatcly dedicated by THE AUTHOR 



\section{PREFACE}

In presenting this book to the medical profession, the author does so with the feeling that it will be of some assistance to that great mass who were as unfortunate as himself in their early college training in the special field of Proctology. Diseases of the Rectum and Anus have been, and still are, in a great many colleges, dismissed with a single lecture or two, delivered as a part of the course on general surgery. The young graduate in medicine leaves his Alma Mater with a hazy idea that occasionally patients may suffer from "Piles or Fistula," and an operation under general anesthesia is their only hope of relief.

The fact that the profession as a whole has been so remiss in the treatment of patients suffering from rectal diseases has left the field in the past to the quack and the irregular.

A few earnest practitioners, however, in different parts of the country, gradually discovered that there was something more to rectal diseases than the treatment of "Piles and Fistula," and commenced the scientific study of the lower bowel with the result, that today the special field of Proctology is firmly established with conscientions workers in all parts of the world. The results of the work of some of these men have been given to the world in the shape of most complete text-books on the subject. In 
many of these works, however, the subject has been treated from the standpoint of the specialist in rectal diseases, and written for those who wish to follow that line of work.

With the introduction of local anesthesia into the treatment of diseases of the Rectum and Anus, a new field of work lias been opened. Those patients suffering from many diseases of this region, who liave sought the advice and care of the irregular and the advertising quack, have done so on account of their dread of hospitals, general anesthesia and "the knife."

In order that the general practitioner may be qualified to diagnose and treat his patient suffering from ano-rectal diseases, as scientifically and as successfully as he does affections of other organs and localities, the author presents the results of his experiences in the treatment of ano-rectal disease.

The diagnosis of disease originating in this region, has been dwelt upon to emphasize the importance of early examination. Illustrations, for the most part original, have been used wherever it has been thought necessary to supplement the text for the sake of clearness.

Non-surgical methods are described in those conditions where thoy have been found of value, and the technique of operative measures under local anesthesia been made as simple as possible. Only those conditions which are amenable to treatment in office practice liave been discussed, and the limitations of office treatment clearly set forth. For information regarding those operative measures which are ouly applicable under general anesthesia, and the consideration of those diseases whose treatment requires confinement to bed, the reader is referred to the 
several complete works on proctology which are now available.

Those physicians living and practicing in the Southern states, particularly, will appreciate the inclusion in this work of a chapter on Dysentery. The anthor has been exceedingly fortunate in securing the services of a man to prepare this chapter, than whom there is no one better posted on the subject: Dr. John L. Jelks, of Memphis, Tenn., Vice-President of the American Proctologic Society.

Inasmuch as a very important index to the condition of the entire digestive tract and its functions is found in the excretions; and the fact that the examination of the stools which is fully as important as the urinary analysis, has been too long' neglected; a chapter on the examination of the feces has also been included. 'Dr. George W. Wagner, of Detroit, Attending Physician to Harper Hospital, Gastro-Enterologist to the German Polyclinic and Clinical Professor of Medicine in the Detroit College of Medicine, has kindly contributed this chapter, and the anthor considers himself extremely fortunate in securing the assistance of so well qualified a man.

To the above named gentlemen ; to Dr. Robert C. Jamieson, of Detroit, Dermatologist to Harper Hospital Polyclinic, who made the excellent photographs under the author's direction; to Mr. James T. Nolan, the artist of Western Reserve University Medical Department, of Cleveland, O., who made all of the drawings; to the J. F. Hartz Co., of Detroit, who furnished the illustratıous of many of the surgical instruments; to the publishers for their hearty and willing co-operation; and lastly, to the many members of the medical profession through 
whose courtesy the author has been able to treat the large number of cases, the results of his experience with which lats furnished the basis for the preparation of this work -the author extends his sincere and heartfelt thanks.

Besides the results of his own experience, the author has availed limself of the privilege of consulting many of the recent works and text-books on the subject of proctology, among which may be mentioned those of Tuttle, Gant, Matthews, Martin, Ball, Cripps, Wallis and Gray, as well as many articles by other authors appearing in the current literature of the day.

If the author has succeeded in so simplifying the diagnosis and treatment of many of the more common diseases of the Rectum and Anus so that this work will be of some assistance to the busy general practitioner, in his every day work; and has assisted in even a small degree in broadening the scope of the use of local anesthesia in this field, he will feel that he has accomplished all that he set out to do. This modest work does not pretend or aspire to take the place of a text-book on the whole subject of proctology, but if it will find a place on the physician's desk as a working hand book, the author feels that it will fill a long felt want.

604 Washington Arcade.

Louis J. Hirichiman.

Detroit, Jan. 11, 1909. 


\section{TABLE OF CONTENTS}

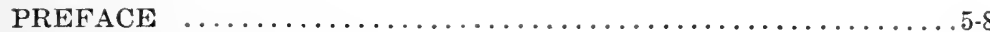

\section{CHAPTER I.}

\section{ANATOMY.}

The Anus-Anal Canal-External Sphincter Muscle-Anal Papillae Crypts of Morgagni-Rectum-Internal Sphincter Muscle-Columns of Morgagni-Rectal Valves-Levator Ani Muscle-IlioCoccygeus - Pubo-Coccygeus - Pubo-Rectalis - Ligaments Ano-Coccygeal and Lateral-Relations of the Rectum-IsehioRectal Fossa-Sigmoid Colon-Blood Supply-Arterial, Venous

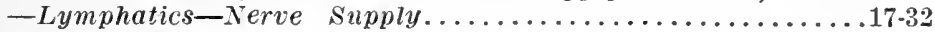

\section{CHAPTER II}

\section{SYMPTOMS WHICH SHOULD CALL ATTENTION TO THE RECTUM.}

Fain - Tenderness - Spasm - Bleeding - Itching - Protrusions - Ulcerations - Discharge - Constipation - Diarrhoea - Altered Stools-Sacral Backache-Shooting Pains down the Limbs -Crampy, Painful and Scanty Menstruation-Frequent and Painful Urination-Loss of Appetite-Impaired DigestionNausea-Headache-Sallow Complexion - Fever-AnemiaRestlessness in Children-Foreign Bodies...............33-40

CHAPTER III.

\section{EXAMINATION OF THE PATIENT.}

The physician's offices-Operating room equipment-Preparation for examination-Interrogation of patient-Record keeping-Sims' position-Ocular inspection-Digital examination-Position of patient, correct and incorrect-Vagino-rectal examinationEversion of anus-Lithotomy position-Bimanual abdominovaginal and abdomino-rectal examination-Squatting position Internal inspection-Knee-shoulder position-Correct and incorrect methods-Anoscopy-Necessary instruments and appliances-Technique of proctoscopy without instruments-Instrumental proctoscopy-Necessary instruments and appliancesExaggerated Lithotomy position-Sigmoidoscopy-Necessary instruments-Congenital defects and mal-formations......41-76 


\section{CHAPTER IV.}

\section{CONSTIPATION AND OBSTIPATION.}

Differentiation of two conditions-Physiology of defecation-Etiology of Constipation-Methods of examination-Diagnosis and treatment-Dietary -Exercise -Mechanical therapeutics-Author's method-Internal Medication-Obstipation-The rectal valves-Rectal valvotomy-A uthor's technique ...........77-96

CHAPTER V.

FECAL IMPACTION.

Etiology-Symptoms-Diagnosis-Treatment-Palliative and radical

\section{CHAPTER VI. \\ PRURITUS ANI.}

Etiology-Local causes - Skin diseases - Reflex- ConstitutionalDietary-Parasitic-Irritation-Examination of patient-Diagnosis-Treatment-Palliative and medicinal-Mechanical-Surgical-Hamilton's operation-Balls operation-Author's technique under local anesthesia......................102-121

CHAPTER VII.

ANAL FISSURE AND ULCER.

Etiology-Location-Sentinel Pile-Diagnosis-Methods of examination-Treatment-Palliative - Non-Surgical-Surgical Treatment-Incision-Divulsion of sphincter-Excision-A uthor's operation-Anal ulcer-Treatment-After care............122-136

CHAPTER VII.

ABSCESS OF THE ANO-RECTAL REGION.

Predisposing causes-Etiology-Tegumentary Abscess-DiagnosisTreatment-Subtegumentary or marginal abscess-SymptomsMethods of Examination-Sub-mucous abscess-ExaminationDiagnosis - Treatment - Operative Technique- After Care Ischio-rectal abscess-Etiology - Symptoms - ExaminationDiagnosis-Treatment-After care-Caution ............137-151

CHAPTER IX.

FISTULA IN ANO.

Etiology-Varieties-Simple, horseshoe and multiple fistulaeBlind, internal and external fistulae-Submucous fistulaeSimple complete fistulae-Symptoms-Examination and diagnostic methods-Treatment - Incision - Excision - Author's technique-Ligature operation-After care-Blind external fistulae-Examination, diagnosis and treatment-Blind internal fistulae-Symptoms - Examination-Diagnosis - TreatmentAfter care-Submucous tract-Mucocutaneous fistula-Bismuth injection-Technique-Fistula in ano in the TuberculousSymptoms-Diagnosis-Treatment ..............152-172 


\section{CHAPTER $\mathrm{X}$.}

\section{HEMORRHOIDS.}

Neglect on the part of medical profession-Incomplete training of medical students in proctology-Varieties of hemorrhoidsEtiology-Anatomical-Habit-Occupation-Abuse of cathartics-Symptoms-Hemorrhage-Pain - Protrusion-Diagnosis and technique of examination-Position of patient-Instruments required-Differential diagnosis-Treatment-Palliative Injection treatment - Cauterization - Operative treatmentTechnique-Author's bloodless operation-Instruments and technique-After care-Incision-Acute thrombotic hemorrhoids-Technique of operation-External integumentary hemorrhoids-Operative technique-After care-Caution.......173-208

CHAPTER XI.

\section{RECTAL POLYPI, HYPERTROPHIED ANAL PAPILLAE, CRYPTITIS.}

Polypi-Varieties-Symptoms-Diagnosis-Treatment-Anal papillae-Description -Etiology- Examination-Diagnosis-Symptoms-Cryptitis - Etiology - Diagnosis - Symptoms - Treat-

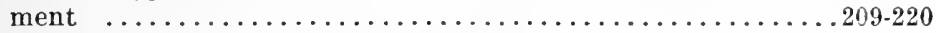

\section{CHAPTER XII.}

\section{PROCTITIS AND SIGMOIDITIS.}

Acute Proctitis-Etiology-Symptoms-Diagnosis-Method of examination-Treatment-Dietary-Systemic-Local-Posture of patient-Apparatus required-Internal medication-Chronic proctitis and sigmoiditis-Hypertrophic and atrophic-Hypertrophic-Etiology-Symptoms-Diagnosis-Treatment- Chronic atrophic proctitis and sigmoiditis-Pathology-Symptoms-

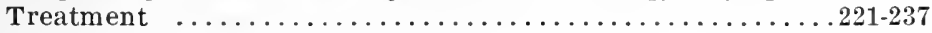

\section{CHAPTER XIII.}

DYSENTERY.

Historical-Geographical distribution-General etiology-SeasonRace-Sex-Hygiene-Topography and condition of soil-Foods -Drinking water-Classification-Acute catarthal dysentery - Special etiology-Pathology-Symptoms - Diagnosis-Prognosis-Diphtheritic dysentery-Etiology-Pathology-Symptoms-Diagnosis-Complications-Secondary Diphtheritic Dys. entery-Symptoms-Prognosis-Amoebic dysentery-Etiology -The Amoeba dysenteriae-Pathology-Symptoms-Complications and Sequelae-Diagnosis-Prognosis-Treatment-Prophylaxis-Diet-Remedies-Irrigations-Technique-Local Applications-Technique-A ppendicostomy-Valvotomy ....238-283

CHAPTER XIV.

\section{PROLAPSE OF THE RECTUM IN CHILDREN.}

Degrees of prolapse-Etiology-Symptoms-Diagnosis-Treatment - Palliative-Prophylactic-Dietary-Concealed prolapse-Description-Education of mothers-Palliative treatment-Cauterization-Technique of operation-After care..........284-294 


\section{CHAPTER XV.}

THE TECHNIQUE OF THE USE OF LOCAL ANESTHESIA IN THE TREATMENT OF ANO-RECTAL DISEASES.

Field of local anesthesia-Anesthetic agents-Technique of preparation-Instruments and apparatus required-Preparation of patient-Posture-Technique of anesthetization-Caution-External hemorrhoids-Acute thrombotic hemorrhoids-Peri-anal abscess-Fissure in ano-Fistula in ano-Hypertrophied anal papilla-hypertrophied rectal valves-Removal of foreign bodies -Removal of peri-anal benign growths-Posterior internal

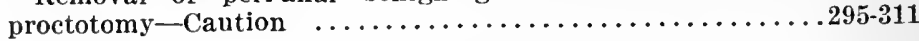

\section{CHAPTER XVI.}

THE LIMITATIONS OF OFFICE TREATMENT AND INDICATIONS FOR OTHER MEASURES.

Limitations of local anesthesia in rectal surgery-Necessity for careful and complete examination-History of concurrent disease, Anemia and hemophilia-Malignancy-Syphilis-Tuberculosis and Hysteria-Early diagnosis of malignant disease-Symptoms of beginning cancer-Ulceration of the bowel-Colostomy - Stricture of the rectum-Nitrous oxide-Circum-anal and peri-rectal abscess-Fistula in ano-Hemorrhoids-Prolapse of the rectum-Removal of concretions or foreign bodies-Fistula between rectum and other organs......................

\section{CHAPTER XVII.}

THE FECES AND THEIR EXAMINATION.

Normal stool-Duration of passage-Amount, consistency and form-Odor-Color-Macroscopic elements-Clinical examination of the stool-Normal constituents-Pathological constituents-Chemical examination-Reaction-Tests-Estimation of albumin residue-Clinical significance of tests-Mucus-Bilirubin-Semi-digested cells-Hyaline cells-Bile pigment-Fat - Remnants of meat-Excess of connective tissue, undigested muscle fibre-Pathologic carbohydrate fermentation and albumin fermentation-Pus-Blood-Various tests-Gall-stonesIntestinal concretions-Intestinal sand-Animal parasitesProtozoa-Worms-Varieties-Character of feces in certain intestinal infections-Acute intestinal catarrh-Chronic inflammation of the intestines-Diphtheritic enteritis-Muco-Membranous colitis-Cholera Nostras-Dysentery-Amoebic dysen.

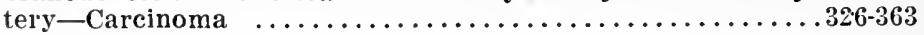




\section{LIST OF ILLUSTRATIONS}

PLATE I. Blood Vessels of the Rectum...................... Figure.

1. Rectum and Anal Canal in the Male................. 19

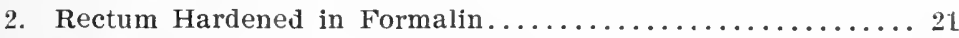

3. Proctoscopic View of Rectal Valves................. 23

4. Muscles and Nerves of Male Pelvic Outlet.............. 25

5. Columbus Operatng Table................... 43

6. Simple Form of Sterilizer for Office Use............... 44

7. Small Instrument and Dressing Sterilizer.............. 44

8. Characteristic Sitting Posture in Ano-Rectal Disease.......45

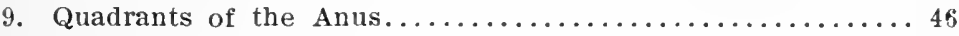

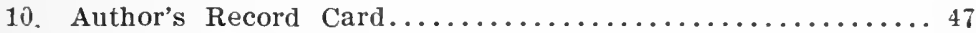

11. Reverse Side of Record Card.................. 48

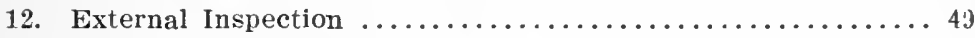

13. Electric Headlight ........................... 49

14 Application of Lubricant from Collapsible Tube......... 50

15. Incorrect Method of Digital Examination............ 51

16. Correct Method of Digital Examination............. 52

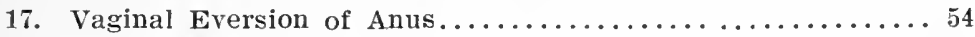

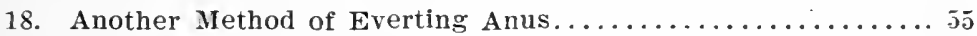

19. Amount of Possible Eversion of Anal Tissues........... 55

20. Recto-Abdominal Bimanual Palpation............... 57

21. Method of Recto-Abdominal Palpation............. 57

22. Palpation of Rectum Through Posterior Vaginal Wall..... 58

23. Method of Examining the Coccyx................ 59

24. Ischio-Rectal Abscess ..................... 60

25. Squatting Position ......................... 61

26. Rubber Bulb Syringe...................... 62

27. Method of Using Author's Fenestrated Anoscope..........62

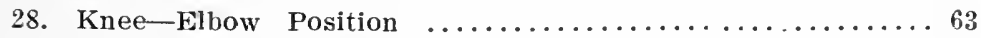

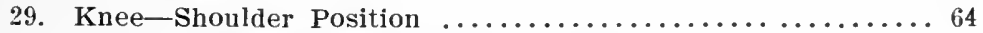

30. Author's Anoscope with Oblique Opening.............. 65

31. Author's Adjustable Fenestrated Anoscope............ 66

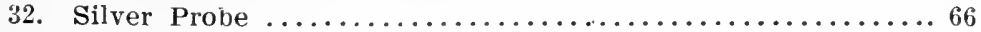


33. Long Alligator Forceps.................... 67

34. Kelly Anoscope $\ldots \ldots \ldots \ldots \ldots \ldots \ldots \ldots \ldots \ldots \ldots \ldots \ldots \ldots \ldots$

35. Bivalve Rectal Speculum.................... 68

36. Wales Rectal Bougie...................... 69

37. Author's Modification of Martin Proctoscope............ 70

38. Exaggerated Lithotomy Position.................. 72

39. Kelly Sigmoidoscope $\ldots \ldots \ldots \ldots \ldots \ldots \ldots \ldots \ldots \ldots \ldots \ldots \ldots$. 7 8

40. Sigmoidoscope with Author's Tilting Obturator..........

41. Atresia Ani Vaginalis (comp'ete) ................ 74

42. Atresia Ani Vaginalis (incomplete) ............... 75

43. Author's Dilating Rectal Massage Bag.............. 86

44. Author's Rectal Massage Bag (inflated and deflated)....... 87

45. Position foi Author's Method of Rectal Massage.......... 88

46. Author's Four-Inch Operating Proctoscope............. 92 .

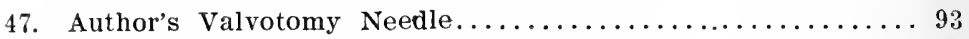

48. Author's Angular Rectal Scissors................ 34

49. Technique of Author's Operation for Rectal Valvotomy ..... 94

50. Proctoscopic View of Author's Valvotomy Operation....... 95

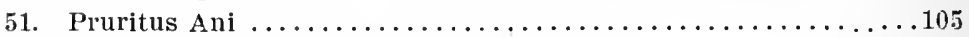

52. External Hemorrhoids with Pruritus Ani............... 106

53. A Simple Rectal Dressing...............................

54. Sharp Pointed Scissors, Curved on the Flat............1 t $_{\mathfrak{t}}$

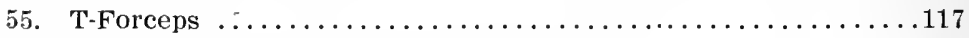

56. Lines for Incision in Ball's Operation.................

57. Dissection of Flap-Ball's Operation............... 119

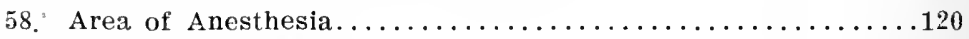

59. Fissure of the Anus. . . . . . . . . . . . . . . . . . . 122

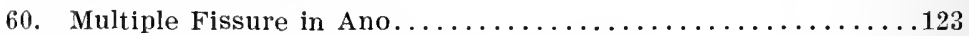

61. Fissure in Ano from Crypt of Morgagni.............125

62. Applying Ointment to Anus from Lead Tube..............

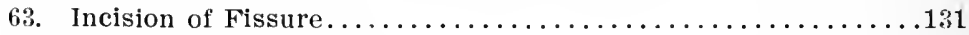

64. Sharp Toothed Forceps..............................

65. Author's Technique for Excision of Anal Fissure.......... 134

66. Operation for Excision of Anal Ulcer.................

67. Ano-Rectal Abscesses ................................ 189

68. Proctoscopic View of Submucous Abscess............... 143

69. De Vilbiss Rectal Speculum................... 145

70. Incision for Opening Ischio-Rectal Abscess.............. 143

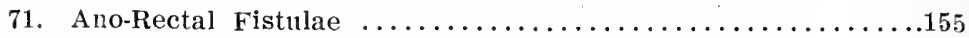

72. Direct Complete Fistula in Ano..................... 156

73. Angular Fistulous Tract...................... 157

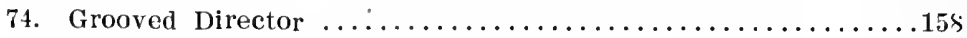

75. Right-Angled Incision for Fistula in Ano............. 160 
76. Author's 'Technique for Excision of Fistulous Tract.......161

77. (a) Technique of Ligature Operation for Fistula............ 163

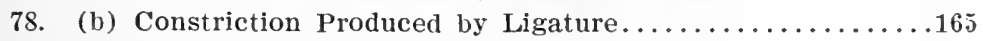

79. Acute External Thrombotic Hemorrhoids...............176

80. External Thrombotic Hemorrhoids.................. 177

81. External Cutaneous Hemorrhoids......................

\$2. Interno-External Hemorrhoids ....................... 179)

83. Section of Interno-External Hemorrhoids . . . . . . . . . . . . 180

84. Interno-External Hemorrhoids, Injected.............. 18.

85. Prolapsing Internal Hemorrhoids.................. 188

86. Method of Injecting Prolapsing Internal Hemorrhoids........195

87. Author's Hemorhoidal Forceps................... 196

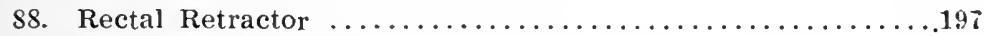

89. Author's Blunt Ligature Carrier...................... 198

90. Technique of Author's Bloodless Hemorrhoid Operation......199

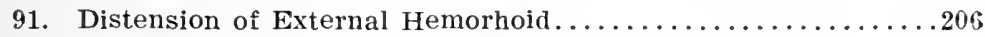

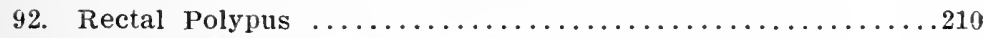

93. Section of Anal Canal, Showing Hypertrophied Papillae.....213

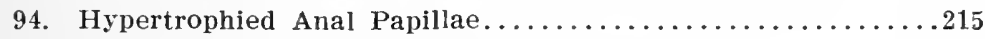

95. Proctoscopic View Hypertrophied Anal Papillae............216

96. Spraying Rectum in Knee-Shoulder Position............225

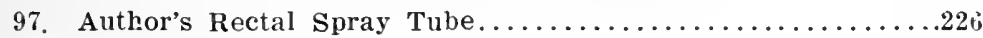

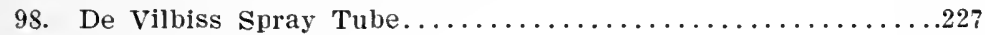

99. Ulcer of the Rectum. . . . . . . . . . . . . . . . . . . 233

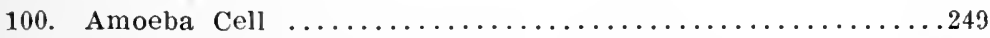

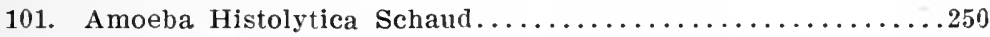

102. Slough of Mucous Membrane from Fatal Case............254

103. Edge of Intestinal Ulcer.....................

104. Dysenteric Ulceration on Valves of Houston............257

PLATE II. Section of Intestine Below Ulceration, Opp......... 258

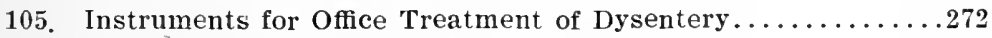

106. Jelk's Soft Rubber Recurrent Irrigating Tube............ 274

107. Exaggerated Sim's Position for Colon Irrigation........... 276

108. Position for Introduction of Colon Tube Through Proctoscope 278

109. Method of Making Local Applications to Rectum...........280

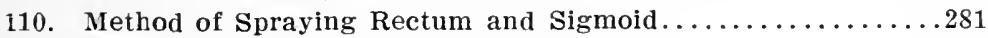

111. Prolapse of Rectum-Third Degree................ 285

112. Cauterization of Prolapse of Rectum-First Degree........292

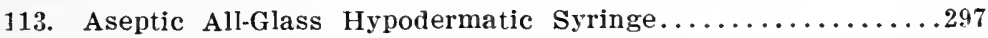

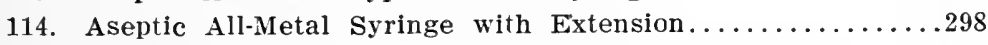

115. Point of Puncture for Injecting Local Anesthetic.......... 300

116. Amount of Distension of Tissues for Local Anesthesia.....301

i17. Dilating Sphincter with Mechanical Vibrator............ 302 
118. Amount of Dilatation Under Local Anesthesia...........303

119. Author's Modification of Martin Operating Proctoscope.....308

120. Cancer of Rectum with Multiple Fistulae...............

121. Proctoscopic View of Rectal Carcinoma.............. 317

122. Carcinoma, Specimen Shown in Preceding................. 318

123. Cancer of the Rectum.............................

124. Cancer of the Rectum-Interior View .............. 320

125. Bismuth Sulphide Crystals from Stools............... 323

126. Collective View of the Feces..........................

127. Muscle Remnants in Feces.......................

1.8. Hematoidin Crystals from Acholic Stools................. 334

129. Acholic Stools . . . . . . . . . . . . . . . . . . . 335

130. Steele's Modification of Strasburger Fermentation Apparatus.337

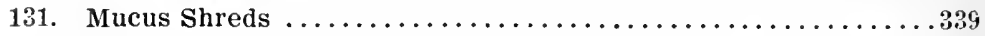

132. Mucus Shreds after Addition of Acetic Acid.............. 340

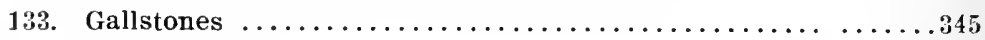

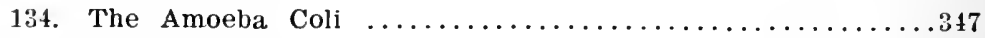

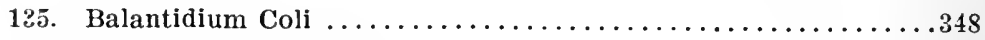

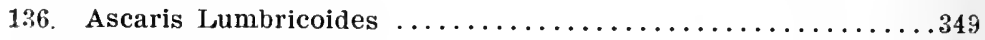

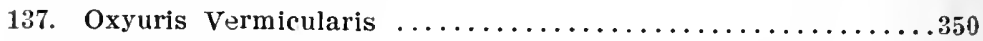

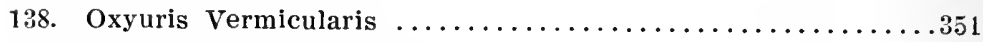

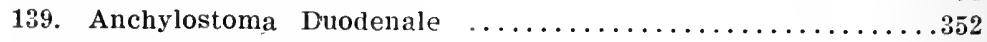

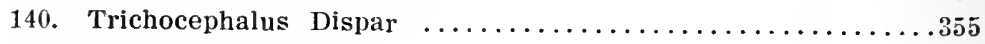

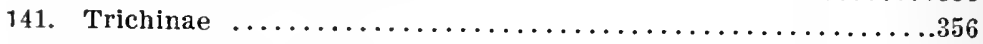

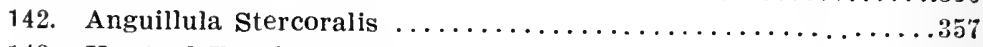

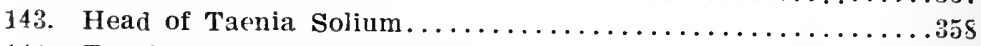

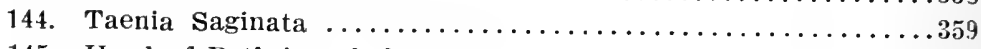

145. Head of Bothriocephalus Latus.................. 360 


\section{CHAPTER I}

\section{ANATOMY.}

It is not the intention in a work of this scope to go into minor anatomical details in the description of the Anus and Rectum. It is essential, however, that one who intends to treat even the more common and uncomplicated diseases of the Anus and Rectum should have a practical working knowledge of the gross anatomy of the ano-reetal region.

In reversing the usual order of describing these organs, the author starts with the Anus first because it is to the anal orifice that one's attention is first directed in pro. ceeding to examine or operate for diseased conditions affecting these organs. It appears to the author, therefore, that the anatomy of these organs should be described in the order in which they are met with: from without, inward.

The Anus. The Anus is an oval aperture, longitudinal when in repose, situated at a point equidistant from the tuberosities of the ischii ; and about one inch anterior to the tip of the coccrx. In the female it is situated a little more anteriorly than in the male. The anus is surrounded by integument which is colored slightly darker than the surrounding skin. The skin around the anus is arranged in radiating folds caused by the contraction 
of the corrugator cutis ani muscle. The circum-anal integument contains sweat glands, sebaceous glands and hair follicles. The circumference of the anal orifice varies from an inch to an inch and three quarters, but it may be dilated to a circumference five or six times greater.

The Anal Canal. The Anal Canal extends from the point at which the sides of the anal aperture first appose to the linea dentata or lower edges of the semilunar valves, which guard the openings to the crypts of Morgagni. Its depth varies from two-thirds to an inch and a quarter. It is lined by a membrane composed of thin transitional epithelium gradually clanging in histological formation from the stratified cells of true skin at the anus, to the goblet cells of mucous membrane at its juncture with the rectum at the linea dentata or anorectal line. Surrounding the lining membrane is one of cellular tissue, and beneath this the muscular layer composed of the external sphincter, a few fibres of the levator ani, and the lower portion of the internal sphincter. The dimensions of the anal canal, when in repose or dilated is slightly smaller than that of the anus itself in like condition. The lining membrane presents to the eye a pinkish red shining appearance; in some cases a more or less purplish hue.

The external sphincter muscle is the most important muscle with which we have to deal from a surgical point of view, and is the principal muscular structure which goes to form the anal canal. It is composed of circular and longitudinal fibres. The longitudinal arise from the lower end and posterior aspect of the coccyx and surrounding the anus in an elliptical manner meet and are 


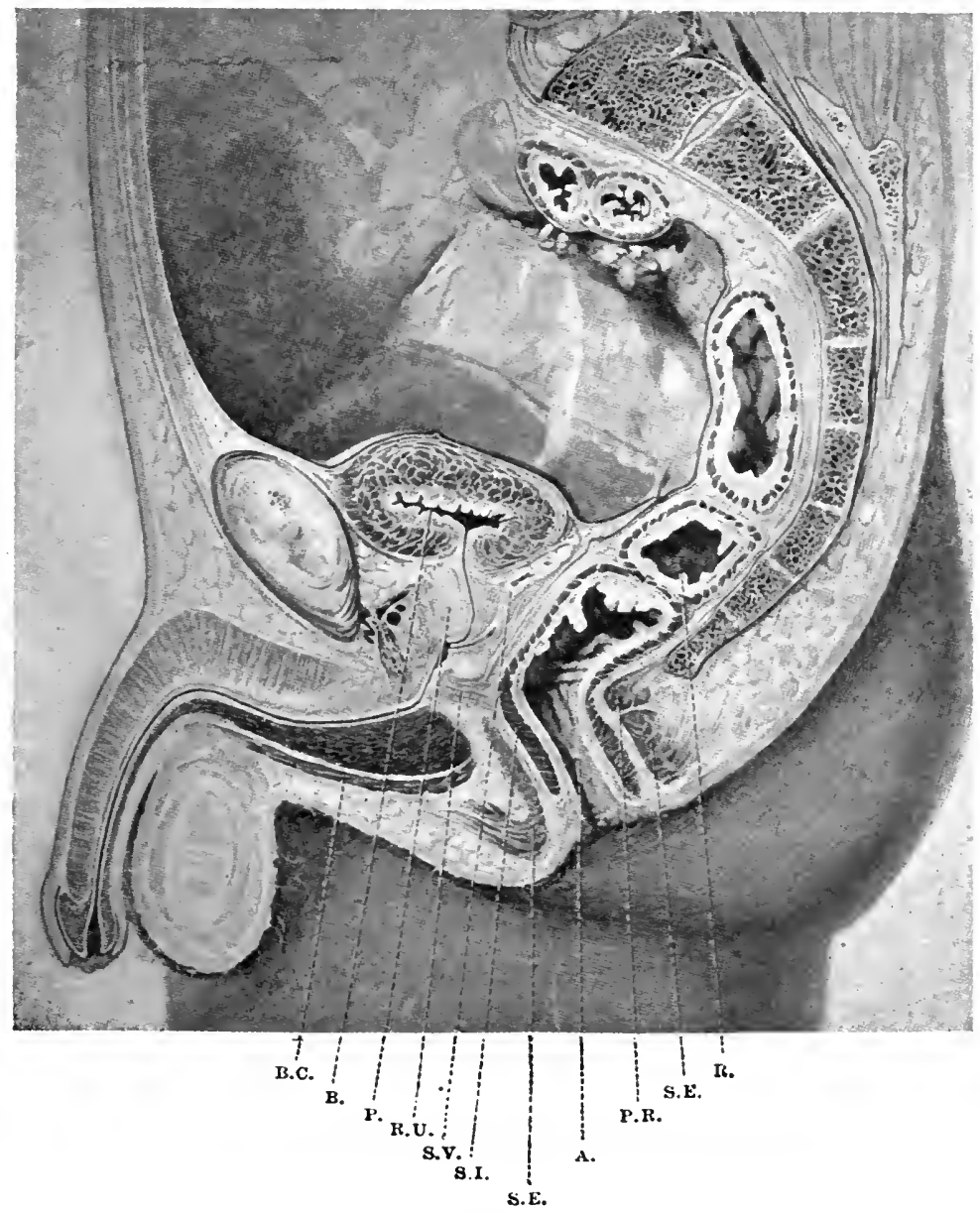

Fig. 1.

The Rectum and Anal Canal in the Male-Longitudinal Section.

Section made by Professor A. F. Dixon of a formalin hardened

B. C. Bulbo-cavernosus muscle. male pelvis.

B. Bladder.

P. Prostate gland.

R. $U$. Recto-urethralis muscle.

$S . V$. Seminal vesicle with ejaculatory duct below.

$S . I$. Internal sphincter muscle.

$S . E$ External sphincter muscle.

$A$. Anus.

$P$. R. Pubo-rectalis muscle round which the rectum bends sharply $R$ Rectum.

-The Rectum; Its Diseases and Developmental Defects. By Sir Charles Ball. 
inserted into the central tendon of the perineum. The circular fibres are more superficial, entirely surrounding the anal canal. The muscle is normally in a state of contraction, keeping the anus closed and it is of great importance in the voluntary control of the act of defecation. Its nerve supply is derived from the third and fourth sacral and superficial branch of the internal pudic and a filament of the fifth and sixth sacral known as the lesser sphincterian nerve. This nerve is of extreme importance in the production of local anesthesia for the dilatation of the anus. It enters the external sphincter on either side at a point at the juncture of the lower and middle third of the anus.

At the upper limit of the anal canal at its juncture with the lower portion of the rectum are situated the anal papillae and crypts of Morgagni. The papillae appear as a more or less distinct line of small saw-tooth like triangular projections which encircle the anal canaı. This line is called the linea dentata, or ano-rectal line Just behind these papillae are found the openings of the crypts of Morgagni. The anal papillae and crypts of Morgagni are of especial interest because they are often the seat of inflammatory conditions which present symptoms often out of all proportion to the size of the lesion arusing them.

The blood and lymphatic supply will be taken up later.

The Rectum. The Rectum is a hollow, tubular organ varying in lengtl from five to seven inches, and extending upward from the ano-rectal line to the recto-sigmoidal juncture. When empty, its anterior and posterior walls appose and a cross-section would show a trans- 


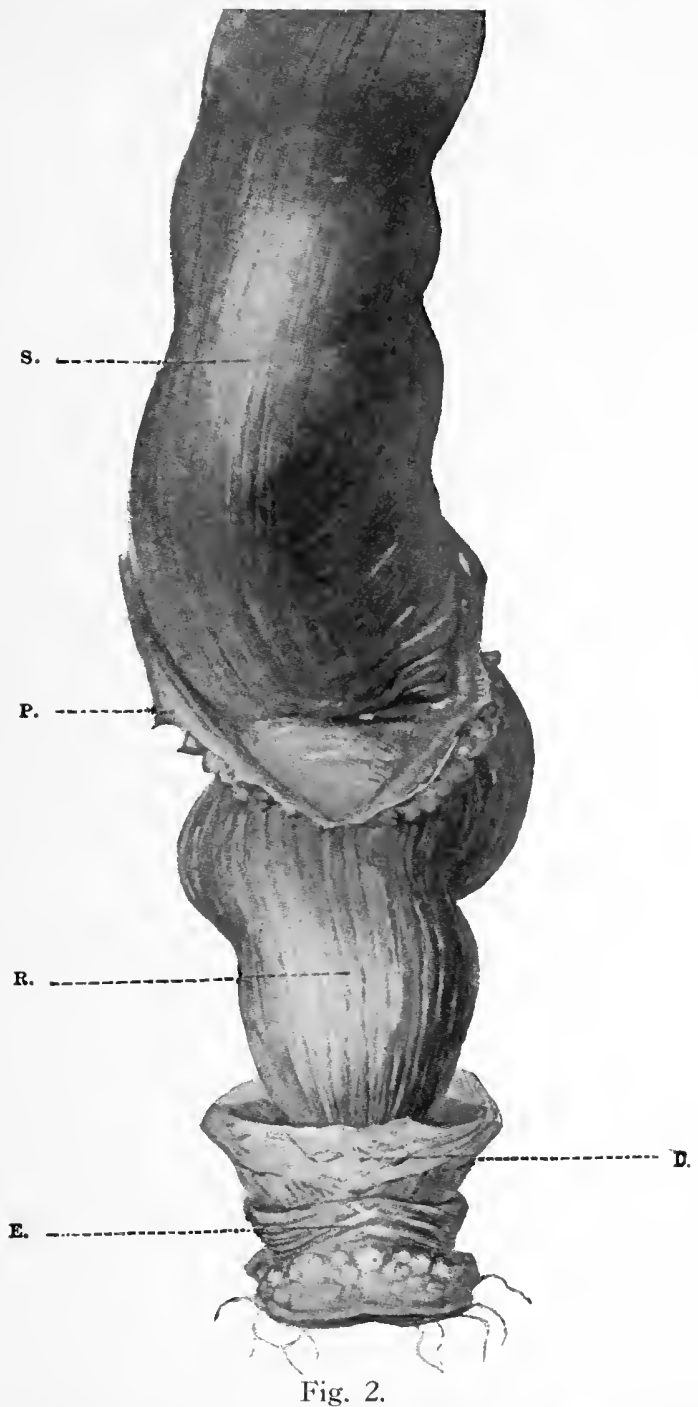

Rectum Hardened in Situ With Formalin and Then Dissected Out. $S$. Sacral curve of rectum.

$P$. Peritoneum cut at reflexion from bowel.

$R$. Portion of rectum uncovered by peritoneum.

$D$. Pelvic diaphragm.

E. External sphincter.

-The Rectum; Its Diseases and Developmental Defects, By Sir Charles Ball. 
verse slit. The rectum is usually understood to be that portion of the lower end of the large intestine which extends from the left sacro-iliac symphysis to the ano-rectal line. Instead of it being a straight canal as its name indicates, it is curved backward from the ano-rectal line, following the hollow of the sacrum, curving forward at the promontory, where it joins the lower portion of the sigmoid flexure. Some authors describe the rectum as that portion which extends from the ano-rectal line to the third sacral vertebra, which includes that portion which is not covered by peritoneum; the part above this being called the lower end of the pelvic colon or sigmoid colon. Inasmuch as this latter division has not been accepted as yet, the author will consider the rectum as described in all of the standard text-books on anatomy. We will consider the rectum as divided in two portions, the upper or peritoneal portion; and the lower or that portion below the third sacral vertebra, the extra-peritoneal. 'Thomas Charles Martin divides the rectal cavity into first, second and third rectal chambers, each cham. ber corresponding to that portion below one of the rectal valves or folds of Houston.

The rectum is composed of four coats, being from within ontward: the mucous, submucous, muscular and serous. The muscular coat is composed of both circular and longitudinal fibres. At the lower portion of the rectum and extending down to the white line of Hilton in the anal canal, the circular muscular fibres are more numerous and thrown together into what is known as the internal sphincter muscle. The mucous membrane is gathered together in folds which converge at the anorectal line, ending at the crypts of Morgagni. These 
folds are known as the columns of Morgagni. With the patient in the knee-shoulder position and the rectum inflated, the circumference of the organ when dilated will vary from five to eight inches. With the rectum inflated certain definite crescentic folds will be seen standing out from the rectal wall, encircling it for from onethird to two-thirds of its circumference. They appear at definite points and are usually three in number. One extremity appears attached lower to the rectal wall than

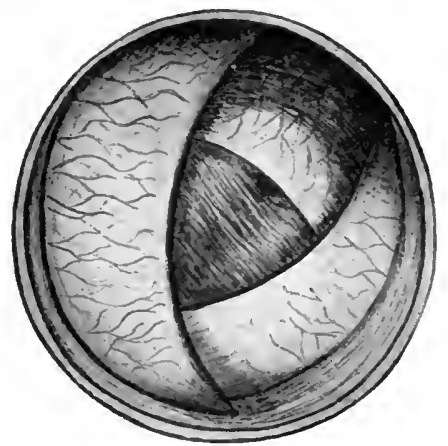

Fig. 3 .

Proctoscopic View of the Rectal Valves.

Semi-diagramatic.

the other, and they are arranged in snch a manner that on proctoscopic view they give the effect of three projecting ledges arranged in the form of a spiral; the second being attached an inch to an inch and a half above the middle of the first; and the third at a point about the same distance above the middle of the second. The first rectal valve, or fold of Houston, as they are called, is situated more often on the left lateral wall of the rectum opposite the location of the prostate gland, while the third is at or below the recto-sigmoidal juncture. These 
valves are not simple folds of mucous membrane, but contain muscular fibres and blood vessels and present all the characteristics of a typical anatomical valve. They are of considerable interest and importance because of the fact that when they are infiltrated, thickened or enlarged, they offer more or less obstruction to the passage of the fecal current, prevent the introduction of the rectal tube, and interfere with the administration of enemata.

Levator Ani Muscle. Outside of the External sphincter muscle, this is the most important muscle with which we lave to deal. With the external sphincter, this muscle practically controls the act of defecation. During defecation the levator ani and external sphincter muscles are relaxed, and the feces are extruded by the involuntary action of the muscular coats of the bowel, assisted by the compression and contraction of the abdominal muscles under the control of the will. The internal sphincter, in all probability does not act as a sphincter at all, but co-operates in the peristaltic movement of the intermal muscular coat of the intestine. When the fecal mass is extruded, the anterior portion of the upper portion of the anal canal is fixed by the recto-urethralis muscle, which is a definite muscular band by which the anterior surface of the bowel at the juncture of the rectum and anus is connected with the urethra. The pubo-rectalis portion of the levator ani then compresses the sides and draws the posterior portion of the opening toward the pubis. The external sphincter then completes the evacuation and closes the anal canal. 


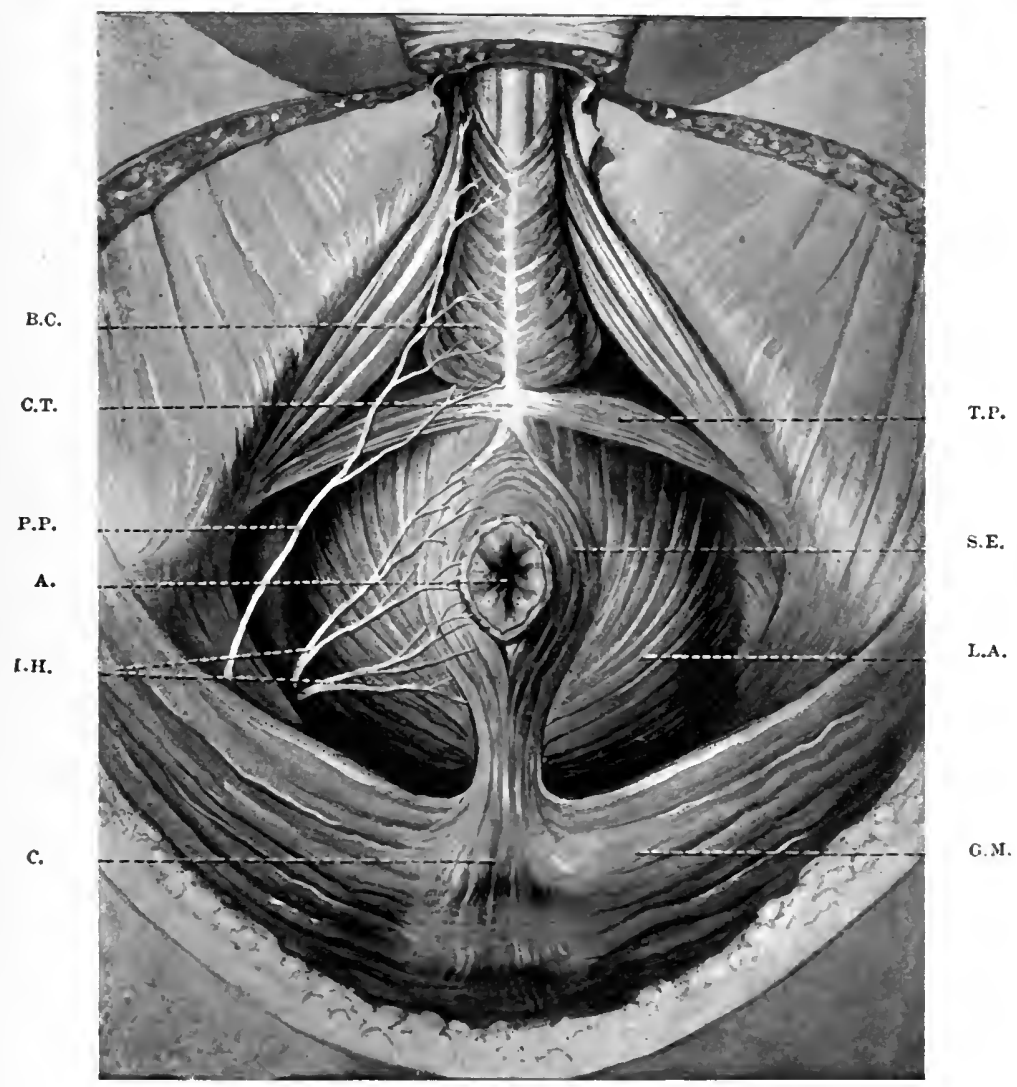

Fig. 4.

\section{Muscles and Nerves of the Male Pelvic Outlet.}

T. P. Transversus perinei muscle.

S. E. External sphincter muscle.

L. A. Levator ani muscle.

G. M. Gluteus maximus muscle.

C. Coccyx.

1. $H$. Inferior hemorrhoidal nerve.

A. Anus.

P. P. Posterior superficial perineal nerve.

C. $T$. Central tendinous point of perineum.

$B$. C. Bulbo-cavernousus muscle.

-The Rectum; Its Diseases and Developmental Defects, By Sir Charles Ball. 
The levator ani, as described by Thompson and Ball, is composed of three main portions, the ilio-coccygeus, pubo-coccygeus and pubo-rectalis.

"Ilio-Coccygeus although definitely attached to the ilium in many lower animals, in man takes origin from the spine of the ischium and from a portion of the obturator fascia, roughly indicated by a white line which extends in a curve from the spine of the ischium to the back of the pubis. Although in older text-books this white line is deseribed as a tendinous origin of the levator ani, recent observations tend to show that but few, if any, of the muscular fibres are actually attached to it, and that it is merely a thickening of the pelvic fascia. From this origin the ilio-coccygeus extends in a fan shape to be inserted into the side of the sacrum and coccyx; it is thin and in part membranous, and must be regarded as a degenerated muscle whose primary function in connection with the tail is lost, but in virtue of its position it contributes to the formation of the pelvic floor. It has no direct relation to the rectum.

"Pubo-Coccygeus arises from the back of the pubis, and also from the obturator fascia, where usually its fibres blend with those of the ilio-coceygens; from this origin the fibres pass almost horizontally back, overlapping the ilio-coccygeus, closely related to the rectum (and vagina), to be attached to the coceyx and ano-coccygeal ligament. A few of the anterior fibres descend in front of the rectum to the perineal body, while lateral fibres are continued down into the aponeurotic sheath which surrounds the anal canal, in which the longitudinal fibres of the external coat of the rectum terminate.

"Pubo-Rectalis, or sphincter recti, is the name given 
by Holl to an important band of fibres of the pubo-coceygeus, which instead of being inserted into the coccyx and its ligamentous connections, is continnous with the fibres of the same muscle on the other side, forming a strong muscular cord round the lateral and posterior aspects of the upper opening of the anal canal. The fibres of the pubo-rectalis muscle arise from the back of the pubis on either side, under cover of the pubo-coccygeus, and pass between the layers of this muscle, with more or less interchange of fibres, to the back of the rectum, where they are continuous with the fibres of the same muscle on the other side. It is the most muscular portion of the levator ani, and when removed from a formalin-hardened body leaves a deep groove posteriorly where the rectum turns abruptly into the anal canal. According to Thompson, although traces of this muscle are found in some lower animals, it is only in the anthropoids that we find a muscular sling strongly developed for the first time, which in man has become evolved into such an importint structure."

Ligaments. The chief ligaments that assist in supporting the rectum are the ano-coccygeal and lateral ligaments. The ano-coceygeal is a cord-like ligament which extends from the tip of the coccyx to a point near the juncture of the amus and rectum on its posterior surface. Attached to it are some of the more superficial fibres of the external sphincter. Upon either side of the rectum, just beneath the lateral reflections of the peritoneum are connective tissue attachments known as the lateral ligaments of the rectum. It is important to remember that they contain the middle hemorrhoidal vessels. 
Relations of the Rectum. The upper half of the rectum is almost entirely surrounded by peritoneum. In front the peritoneum dips down between the rectum and -bladder, forming what is known in the male as the rectovesical pouch, in the female the uterus and vagina take the place of the bladder and the pouch is known here as Douglas' pouch. The distance between the anus and the deepest point of dipping of this pouch is of great importance in the surgery of this region, and the distance varies according to the measurements of different authors. The average distance is given as four inches. Cripps, after careful measurements of a large number of cadavers, gives the distance as $21 / 2$ inches when the bladder and rectum are emptied, and $31 / 2$ inches when both are distended. From this lowest point on the anterior surface of the rectum, the peritoneum gradually invests more and more of the rectum until its upper portion at the posterior wall of the rectum is about $11 / 2$ inches higher than the anterior. Where the two folds of peritoneum come together behind the rectum, it forms a complete mesentery which is continuous with that of the sigmoid.

Other relations of the rectum are in front with the bladder, seminal vesicles, vas deferens, urethra, prostate in the male and the vagina, uterus and adnexa in the female. Posteriorly it lies against the hollow of the sacrum and the coccyx. Laterally its upper portion is oftentimes in close contact with coils of the small intestime when they descend into the pelvis. On either side of the lower half of the rectum are located the ischio rectal fossue.

Ischio-Rectal Fossa. The ischio-rectal space or fossa is a triangular space filled with loosely organized con- 
nective tissue and fat, situated on either side of the rectum between it and the tuberosity of the ischium. The apex of the cavity is directed upward and the base towards the perineum.

Gant describes these fossae as follows: "Their depth varies from one and a half inches in front to two inches behind, and at their lowermost and broadest part they are a little more than an inch in width. Internally these spaces are in relation to the external and internal sphincters, coccygeus, and levator ani muscles; extermally with the tuber ischii and obturator fascia; anteriorly with superficial and perineal fascias; and posteriorly with the border of the gluteus maximus muscles, the investing fascia of which is continuous with the great sacro-sciatic ligament. Within a sheath formed by the obturator fascia are to be found the internal pudic artery, veins, and nerves. The inferior hemorrhoidal vessels and nerves pass through the central portion of the ischio-rectal fossae on their way to the anal canal to which they are distributed, while in the anterior portion of these spaces are the superficial perineal vessels and nerres. The fat and comnective tissue filling these spaces act as elastic supports for the rectum and are largely responsible for the lateral walls of the rectum remaining in contact. These fossae are of surgical importance because of the frequency with which abscesses and fistulas are found in this locality.",

The Sigmoid Colon. The sigmoid colon is that portion of the large intestine extending from its juncture with the rectum at the left sacro-iliac s!mphysis to a point opposite the crest of the ileum where it becomes continuous with the descending colon. It derives its name of 
sigmoid colon or flexure from its double curve. It is entirely a peritoneal organ and is attached by a mesentery which is known as the meso-sigmoid. Its average length is from 18 to 20 inches. When empty the sigmoid colon lies in the left iliac fossa and a portion of it may dip down into the pelvis. When filled, it may extend over and occupy the right iliae fossa as well as the left. It is composed of four coats corresponding to those of the rectum and in addition has on its onter surface directly opposite to its mesenteric attachment a longitudinal muscular band. Its marrowest portion is at its juncture with the rectum. On account of the length of its meso-colon, the sigmoid is of importance because of its tendency in some cases to prolapse or become invaginated into the rectum.

Blood Supply. (See frontispiece.) The arteries of the rectum are the superior, middle and inferior hemorrhoidal, and occasionally a branch from the middle sacral and the vesical. The largest and most important vessel is the superior hemorhoidal which is a direct contimuation of the inferior mesenteric. This vessel, which is situated at the posterior portion of the rectum, shightly to the left of the median line, passes down from the mesentery of the sigmoid colon to the mper portion of the rec. tum at a point about 4 to $4 \frac{1}{2}$ inches from the anus. It here divides into two main branches, the right and left, which almost immediately subdivide into three or four smaller branches, which rom down the rectum almost to the anus, connected by a number of anastomotic branches, some of which pass in through the muscular coat of the bowel to the submucous coat where they end in a number of terminal branches, 
one being usually found in each of the columns of Morgagni. The middle hemorrhoidal artery arises from the internal iliac and enters the rectum on either side through the lateral ligament, where it breaks up into a number of branches which supply the outer coats of the bowel but not the mucous membrane. The inferior or external hemorrhoidal arises from the internal pudic and passing through the ischio-rectal fossa, is distributed to the muscles of the anal canal. 'This artery supplies the cutaneous portion of the anus, the skin surrounding the margin of the anus, but not the mncous membrane.

Venous Supply. The veins of the rectum follow the arteries. The superior hemorrhoidal vein returns the blood from the rectum into the inferior mesenteric vein and directly to the portal circulation. Like the rest of the portal system, the superior hemorrhoidal vein is not supplied with valves. The middle and inferior hemorrhoidal veins return the blood from the anus and circumanal region by way of the internal iliac into the general venous circulation. The hemorrhoidal plexus is composed of a large number of anastomosing veins situated in the submucous and subcutaneous tissues of the anal canal, and is emptied largely by the superior hemorrhoidal veins.

Lymphatics. The lymphatic vessels from the mucous membrane of the rectum proper communicate with a number of small glands known as the post-rectal glands, lying between the rectum and the sacrum, from which lymphatic vessels pass up into the mesentery of the sigmoid. The lymphatics from the skin of the anus and circum-anal region communicate by the immer surface of the thighs with the inguinal glands. An important point to remem- 
ber in this connection is that early involvement of the inguinal glands would indicate disease either malignant or infectious situated in the anal region, while infectious or malignant diseases of the rectum proper would extend to and infiltrate the pre-sacral, lumbar and mesenteric glands.

The Nerve Supply. The rectum is not supplied with sensory nerves, particularly in its upper half. The anus and anal canal and lower portion of the rectum, on the contrary, is liberally supplied. This accounts for the comparative absence of pain when the rectum proper is diseased, and the intense suffering caused by any lesion in the anal canal. The sensory nerves of the anus are derived from the sacral plexus. The external sphincter muscle receives its nerve supply by branches from the sacral plexus, especially the third and fourth nerves. The lesser sphincterian nerve of inorestin which is of great importance in the production of local anesthesia for the dilatation of the external sphincter, is described by Tuttle as: "A filament coming off from the 5th and 6th sacral nerves which passes down the hollow of the sacrum through the levator ani muscle and the rectococygeus ligament, finally reaching the posterior superficial surface of the external sphincter muscle." The levator ani is also supplied by branches from the sacral plexus. While the anus and rectum both receive their nerve supply from the sympathetic and cerebro-spinal systems, the principal nerve supply of the rectum proper is sympathetic, it receiving branches from the mesenteric, sacral and hypogastric plexuses. From the cerebrospinal system it is supplied by some filaments from the third, fourth and fifth sacral nerves. 


\section{CHAPTER II}

\section{SYMPTOMS WHICH SHOULD CALL ATTENTION TO THE RECTUM.}

It has been estimated that one patient out of every seven is suffering from some disease, the relief of which would be assisted, or entirely accomplished, by the treatment of pathological conditions discovered only upon rectal examination. Many patients consult a physician whose localized pain, tenderness, irritation, or other symptoms, call attention at once to the ano-rectal region. Many other symptoms, however, of a more general character, such as disturbances of digestion, menstruation, and the functions of the urinary organs, as well as headache, backache, sciatica, anemia, and sometimes even asthma and acne vulgaris, are more remote evidences of diseases.originating within the confines of the lower bowel.

Pain is the most frequent symptom which causes a patient to seek a physician's aid. It may be located at the anal orifice, in the anal canal or the lower two inches of the rectum. It may be sharp, coming on suddenly, paroxysmal, burning, throbbing, or of a dull aching character. The character of the pain and the time of its onset with relation to the bowel movement, is important, as it, 
of itself, is often a clue to the diagnosis. Sharp, acute pain, of a cutting, burning or stinging quality, coming on with the stool or following it, almost invariably points to some lesion in the anal canal. Sudden, darting pains, oceuring in the intervals between stools, also point to the same region for their origin. Pain of a throbbing character indicates acute, or sub-acute inflammatory conditions. These may be either integumentary, peri-anal, or peri-rectal abscesses. In these latter conditions, a rise in temperature will be noted, and the blood count will show a leucocytosis. Pain of a dull aching character, whether intermittent or constant, may be caused by liemorrhoids, prolapse, polypus, fistula, nlceration of the rectum, benign growths such as rectal adenoids, or malignant disease.

Many diseased conditions of the rectal cavity may progress to an astonishing degree without causing any local pain on account of the lack of sensory innervation of this region. Pain, however, referred to other portions of the body, such as the sacrum, uterus, vagina, bladder, urethra, penis, scrotum, or down the sciatic nerves, or up into the inguinal region, is frequently caused by pathological conditions in the rectum, which cause no local pain whatever.

Tenderness in the circum-anal region, usually points to alsscess formation, or fistula. Tenderness of the anus indicates inflammatory conditions or ulceration.

Sprsm is caused by anything which irritates the sphincter muscles, Anal fissure, ulcer or abscess, as well as hypertrophied papillae, or foreign bodies, are the usual causes of anal spasm.

Bleeding is one of the most frequent symptoms ac- 
companying diseases of the anus and rectum, and it is one of the symptoms above all others which should call for complete examination of the anus, rectum and sig. moid. Bleeding is more common in adults than in children. It may be very profuse, or slight, as simply a drop or two. It usually occurs during defecation, but may occur during the intervals as well. The blood may be discharged either liquid or clotted. It may be pure, or mixed with mucus, pus, feces, or other debris. Fresh blood discharged from the anus, is usually from a local hemorrhage, but may have descended from the sigmoid or colon. The darker in color the blood, the higher in the bowel its source. Rectal hemorrhage may be caused: (1) By local disease; (2) by traumatism ; or (3) following operation. The cause of the last is so evident that it will not be considered and trauma will simply he mentioned. The local diseases of the rectum which may cause hemorrhage are: (1) Internal liemorrhoids. (2) Prolapse. (3) Fissure. (4) Ulceration. (5) Stricture. (6) Malignant disease. (7) Proctitis. (8) Fecal impaction. (9) Polypus. (10) Villous growths. (11) Chancroids and chancres. (12) Condylomata. Other diseases causing local rectal hemorrhage are: (1) Amoebic dysentery. (2) Intussusseption. (3) Embolism of mesenteric artery. (4) Congestion of the portal vein.

The general systemic diseases such as Malaria, Scurvy, Tuberculosis, Typhoid Fever, and otlers, which may during their course give rise to bloody stools, are not considered in this work because the diseased condition is very evident long before the hemorrhage presents itself It may be mentioned, however, that the passage of some mucus streaked with blood in typhoid fever is often a 
warning signal of impending hemorrhage, and perforation.

The type of hemorrhage characteristic of the various conditions will be taken up as each variety is discussed in its respective chapter. 'The author has seen so many cases of Cancer of the rectum, which had gone on to ahmost complete occlusion of the rectum and involve. ment of other organs; whose lives might have been saved if proper and complete examination of the rectum had been made when hemorrhage first manifested itself, that he is constrained to lay great stress on the importance of this symptom. Rectal hemorrhage, no matter how slight, should never be taken for granted as diagnostic of hemorhoids or any other disease, but should call for a complete examination, the technique of which will be explained in the following chapter.

Itching of the anus or of the perineum, scrotum, or vulva, is a frequent accompanying symptom of many anal and rectal diseases. In fact it may occur with any of them. The degree and severity of the itching varies from a slight feeling of uneasiness and irritation, a mild pricking sensation following stools, to the most intense, persistent, aggravating condition characteristic of the more severe types of pruritus ani. Many constitutional diseases, such as Diabetes, and Uric acidosis predispose the patient to itching of any part of the body. When such a patient has a diseased condition of any part of the ano-rectal region, howerer slight, he usually develops pruritus ani in addition to his other symptoms. In the author's experience, ahmost every case showing itching as the predominating symptom has been demonstrated to 
have had its origin in some local diseased condition of the ano-rectal region.

Protrusions. While the most common protrusion of which the patient complains is some variety of hemorrhoids; it should be born in mind that there are several other conditions made manifest by protrusion at the anal orifice, among which may be mentioned: Prolapsus, Polypi, Hypertrophied Papillae, and Cancer. In questioning a patient regarding a protrusion, one should find out whether they appear with stools or not; and whether or necessary to produce it, or whether it appears spontaneously; whether it can be replaced, and if so, whether easily or not. One should inquire as to their number, whether they appear with stools or not; and whether or not their appearance or replacement is accompanied by pain.

Elevations found in the peri-anal region may be smooth and rounded; rough, hard, or soft and fluctuating; and are caused by external hemorrhoids, abscesses, lipomata, condylomata, or the external opening's of fistulae. A rounded elevation occurring at one side of the anus, accompanied by pain of a throbbing character with some rise of temperature, will be found due to a marginal or ischio-rectal abscess. A hard, rounded protruberance, occuring suddenly at the anal margin, accompanied by intense throbbing pain, will be found to be an acute thrombotic external hemorrhoid. A cluster of small, rough elevations at the anal opening, usually posterior, is the most common manifestation of condylomata.

A small papular elevation anywhere in the peri-anal region from which a purulent discharge exudes, is almost invariably the external opening of a fistula. 
Discharge. A listory of discharge from the anus should always suggest a proctoscopic examination. Hemorhage has already been described above. While mucus may be caused by any irritation, acute or chronic; and accompanies practically all forms of rectal disease; it may originate in some inflammatory condition of the colon. The sigmoid should therefore always be explored when a mucous discharge is met with. Puruleut discharge may come from colitis, but more often points to abscess, blind internal fistula, rectal ulceration, or malignant disease. The odor which accompanies the discharge caused by the last mentioned condition, is almost diagnostic in itself. Many patients who complain of pruritus, or local irritation of the anal region, will also complain of the moisture of the parts. It is well to bear in mind the possilility of disease of the Morgagnian erypts as the origin of this symptom.

Constipation. No case of constipation, particularly of the chronic variety, should ever be treated until a complete eximination las been made. So many cases of so-calied constipation, which is purely a functional condition, are in reality due to mechanical causes. Enteroptosis, floating kidney, prolapse, stricture, hypertrophied rectal valves, enlarged prostate, uterine displacements, adhesions, rectocele, perineal lacerations, fecal impaction, and many other diseased conditions, often act in a purely mechanical way, causing obstipation, which can only be discovered after proper examination.

Diarrhoea. Chronic diarrhoea per se, or alternating with constipation, so frequently occurs as a symptom of carcinoma and ulceration, that these diseases should be excluded by examination before treatment is commenced. 
ALTERED STOOLS-SACRAL BACKACHE-SHOOTING PAINS. 39

Persistent diarrhoea, unaccompanied by pain, ocemring in an apparently healthy individual, is very suggestive of beginning malignant disease.

Altered Stools. Deviations in the normal appearance of the stools are often very suggestive. The large, hard stool of prolonged fecal retention, giving a vastly different meaning than the narrow tape-like or pipe-stem stool of stricture. The color, consistency and amount of the stool, as well as the appearance of blood, pus, or mucus with the movement, as has been noted above, are all of importance.

Sacral backache is often the only subjective symptom of begimning malignant disease. It often accompanies internal hemorrhoids, prolapse, impaction, and various benign growths. It is a ssmptom which should always call for rectal examination. Many obstructive conditions of the sigmoid, as well as sigmoiditis, and fecal impaction, will often cause a sense of weight or constriction in the pelvis. When this occurs in females, and disease of the uterus or adnexa are excluded by grnecological examination, the sigmoidoscope should be used.

Shooting pains doun the limbs, particularly the left, may accompany all forms of rectal disease. Sciatica has been so perfectly simulated by rectal ulcer that diagnosticians have repeatedly been led astray. This is often the predominating symptom in lateral ulcer of the rectum. Ischio-rectal abscess, particularly of the left fossa, frequently causes pains shooting down the limbs.

Crampy, painful, and scanty menstruation occurring in women who have perfectly normal genital organs will be found upon rectal examination to be due in many 
cases to ulceration of the anterior rectal wall, fissure, or hemorrhoids.

Frequent and painful urination, pressure symptoms in the bladder, pain and burning at the vesical neck, enuresis: all may be due to a number of anal and rectal diseased conditions. Fissure and ulcer are the most frequent causes of. bladder irritability.

Loss of appetite, impaired digestion, nausea, headache, sallow complexion, and fever are frequently some of the symptoms of an auto-intoxication caused by some interference with the functions of the lower bowel, whose cause will be found upon rectal examination.

Anemia. Persons suffering from anemia should always be questioned as to the existence of rectal hemorrhage as not infrequently the loss of blood from internal hemorrhoids, or ulceration is so extensive as to account for the anemic condition.

Restlessness in Children. When children are restless at night and are continually picking at the nose or scratching the anus or genitals, an examination of the rectum will often disclose the presence of pin worms.

Foreign Body. The history of the swallowing of a foreign body such as a pin or a fish bone, followed in a few days by anal pain or tenesmus, should call for a rectal examination, and the offending cause of the trouble will be found not infrequently protruding from the month of one of the Morgagnian crypts. 


\section{CHAPTER III}

\section{EXAMINATION OF THE PATIENT.}

The first and most-important consideration is the location and arrangement of the examining room. The ideal suite of offices should include besides a reception room, a consultation room, an examining or operating room, and a resting or recovery room. These two latter rooms should be situated at some distance from the reception room and should be separated from the other rooms by walls which are sound proof. It is not a pleasant prospect for a patient in the reception room nervously awaiting his or her turn, to overhear through flimsy plaster or glass partitions, the recital of another's ailments, or the weeping of a hysterical patient on the operating table. Where a glass partition is all that separates the operating room from the reception room, those in waiting are often treated to a shadowgraphic representation of the performance going on within.

One who expects to do minor surgery or treatment work should equip himself properly for the same. A properly fitted-out and furnished operating room should be provided, which could also serve as an examining room as well. The room should be large enough so as not to be uncomfortably crowded with the furniture and 
paraphernalia necessary, and yet small enongh to be compact. The floor should be of tile or granolithic material so as to be water tight and easily cleansed. The walls should be tiled, enameled, or treated with some material that will stand scrubbing. All corners should be rounded off and as little woodwork as possible should enter into its construction.

The location of the suite will depend largely mpon the location of the building itself, but where there is a choice, it will depend upon whether the strongest light is desired in the forenoon or afternoon. Heavy shades should be provided so that the room may be darkened when artificial light is to be used. The walls and everything in the room, as far as possible, should be white. White always gives the patient an impression of cleanliness at once; and the slightest soiling is so conspicuous that they must be-kept clean.

The furniture necessary consists of a surgical table, or chair, which can be adjusted to various positions; an aseptic glass and metal instrument case, glass top instrument table, revolving stool, sterilizer stand, foot tub; with enameled bowls and dressing basins, pail, compressed air tank, and plumbing, electric light wiring, and other fixtures according to the ideas of the individual.

If it is not possible to have a toilet room adjoining the operating room, a commode should be added to the equipment. A retiring or recovery room is almost a necessity as well.

The author prefers an examining table to a surgical chair. He believes that it is not more distasteful to the patient to get up on a table to be examined, than it is to be seated in a chair and by the turn of a crank, to be 


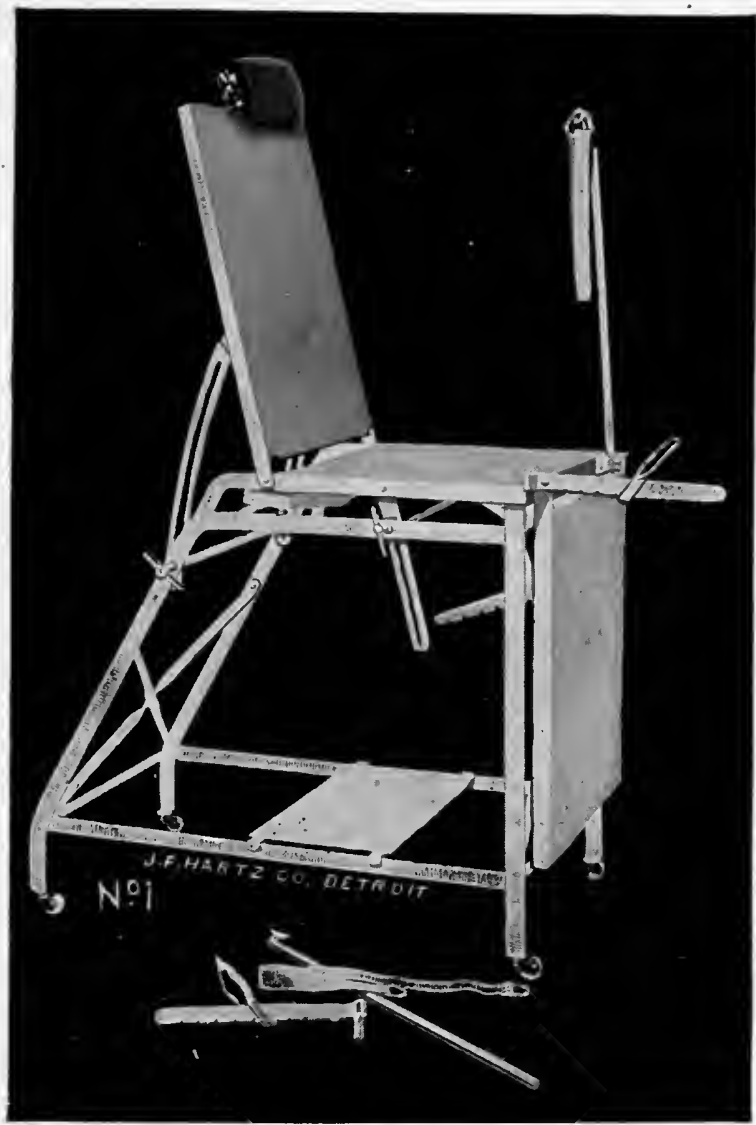

Fig. 5.

Columbus Operating Table.

This is a light but strong all metal operating table, particularly adapted for office work. It may be thrown into any position that either surgical chair or table can be.

jerked or jarred, or flopped into position. Surgical chairs are cumbersome and always getting out of order, and are not to be compared with a nice, clean operating table of white enameled iron which can be adjusted to any 
position required. Hair-stuffed cushions covered with white rubber and not exceeding one inch in thickness are placed on top of the table. The cushions should be thick enough so as to counteract the hardness of the table, and yet not so thick that the patient's buttocks sink down into them.

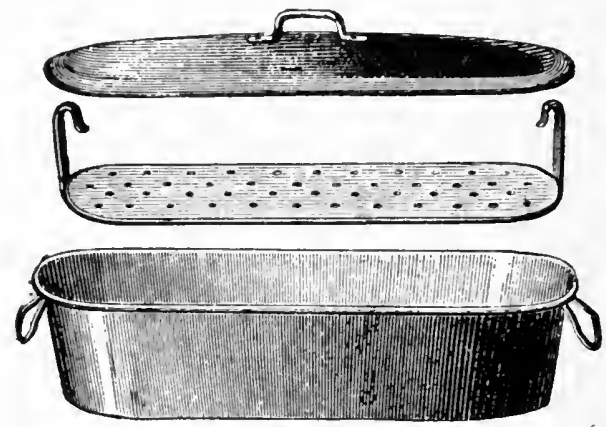

Fig. 6.

A Simple Form of Instrument Sterilizer for Office Use.

Plenty of clean white sheets should be always on hand and the examiner will find it more comfortable and cleanly to wear a white cotton coat such as are commonly worn

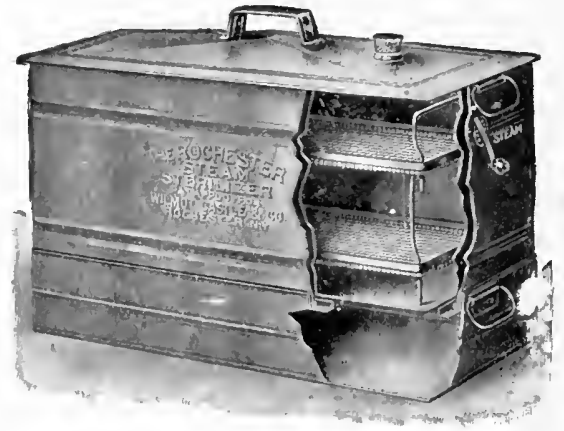

Fig. 7.

A Small Instrument and Dressing Sterilizer.

This is a very simple and popular form of steam sterilizer. The dressings for an office operation may be sterilized in the trays above the boiling instruments. 
by dentists. The author has found the electric head light very useful where the interior of the rectum is to be examined, and believes it so far superior to the head mirror and lamp that he no longer uses the latter.

While it is extremely desirable to have such an equipment, as has been described above, a very satisfactory examination can be made on any sort of a table or bed

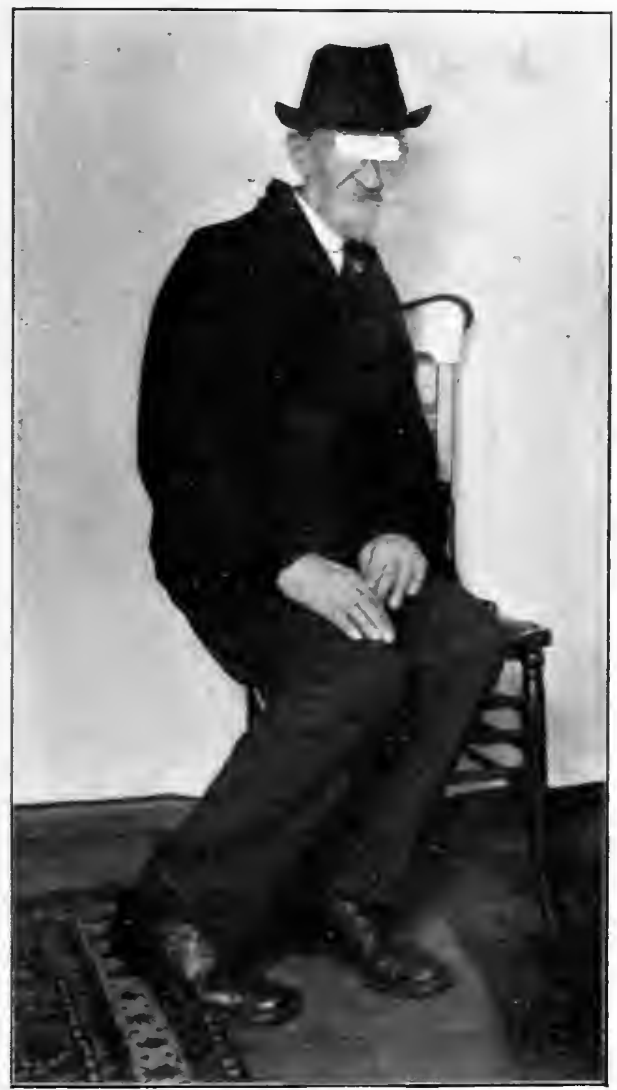

Fig. 8.

Characteristic Sitting Posture Assumed by Patients Suffering from Ano-Rectal Disease. 
with the aid of a good light. The technique which the author uses will be described, not because it will be found the best by all practitioners, but because he has found it the best and most satisfactory in his experience.

The patient should first be asked into the consulting room, and in order to put him at his ease, he should be allowed to tell the story of his ailments in his own way. As he mentions symptoms or salient points which are pertinent, they should be noted down for use in

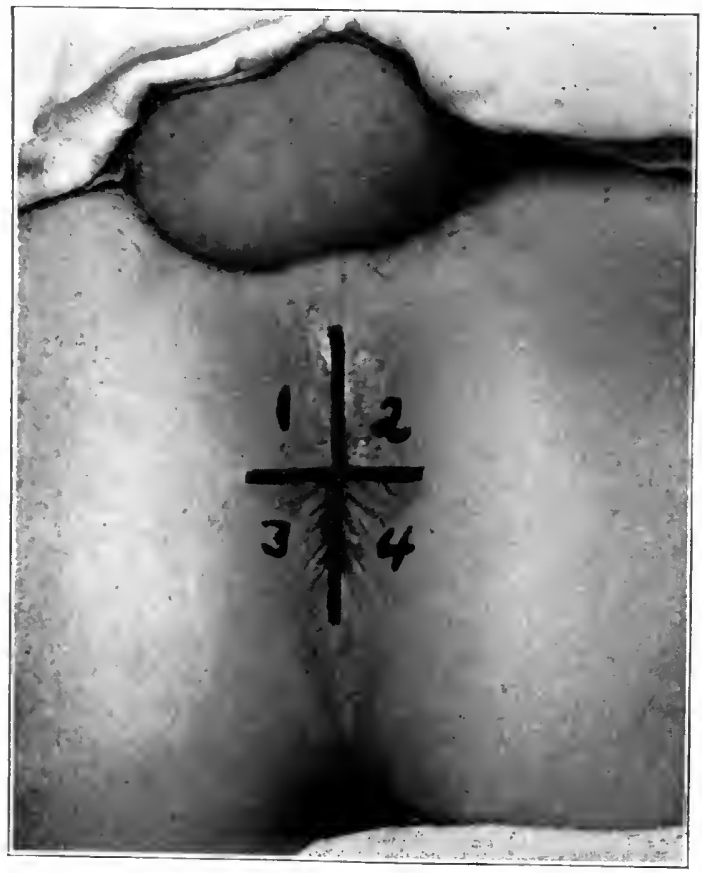

Fig. 9.

The Quadrants of the Anus.

1. Right anterio-lateral quadrant.

2. Left anterio-lateral quadrant.

3. Right posterio-lateral quadrant.

4. Left posterio-lateral quadrant. 
questioning him later. When he has finished, he should be questioned in a more systematic manner, and lis history noted on a special blank or eard kept for the pur-

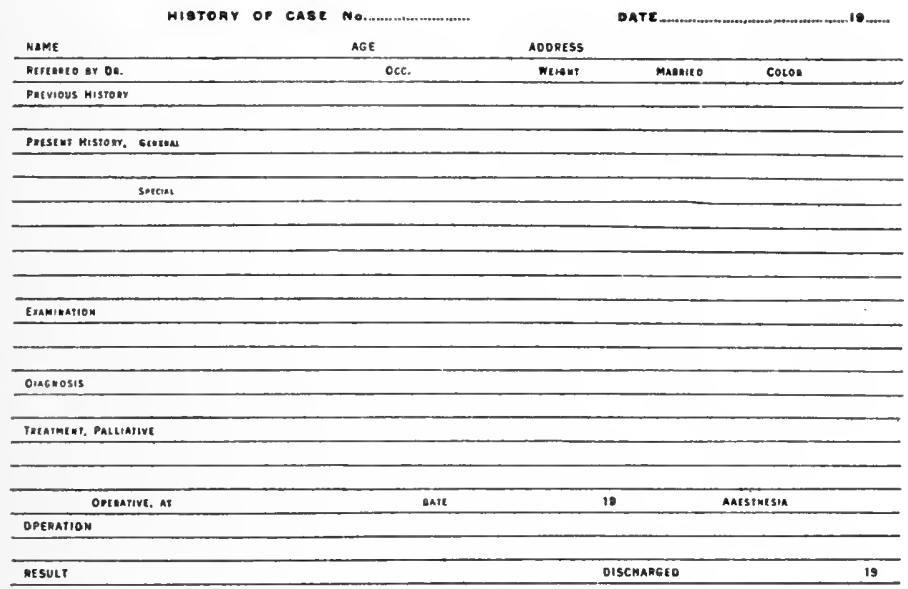

Fig. 10.

A Simple Form of Record Card Used by the Author.

Actual size, four by six inches.

pose. The varions symptoms brought out in this way, will often suggest a tentative diagnosis, but as has been stated in the preceding chapter, nothing should be taken for granted and a complete rectal examination insisted upon. The patient is then taken into the examining room and prepared for the examination. All clothing, corsets, tight waist bands, or anything which constricts, or has a tendency to interfere with respiration, or to crowd the abdominal organs, or intestines out of place, should be loosened or removed. The patient is then placed on the 
table in the left lateral or Sims' position and covered with sheets in such a manner that there is never any unnecessary exposure.

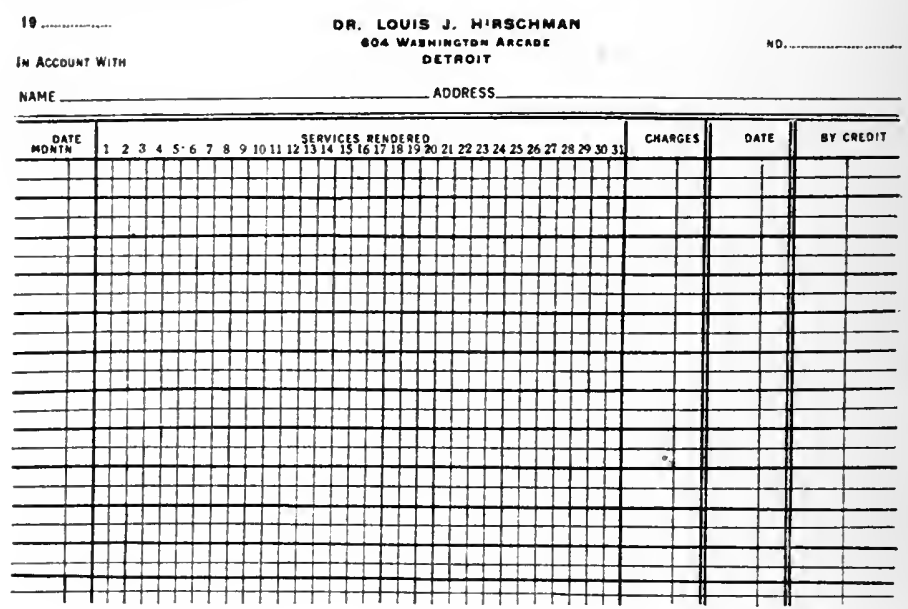

Fig. 11.

Reverse Side of the Preceding on Which the Account with the Patient Can Be Kept.

Size, four by six inches.

With the patient so placed as to get good day light, or by the aid of the head light, the anus, perineum, buttocks, and the genital organs are carefully examined. Discolorations, protrusions, elevations, swellings, abrasions, cracks, skin eruptions, crusts, scars, discharge, or any other abnormal appearances of the parts should be carefully noted.

With the patient in the same position, digital examination is next in order. It is well to have in readiness a bowl of some antiseptic solution, preferably one which will not attack steel instruments. The author has found a $1-10,000$ solution of mercuric iodide the most satisfac- 


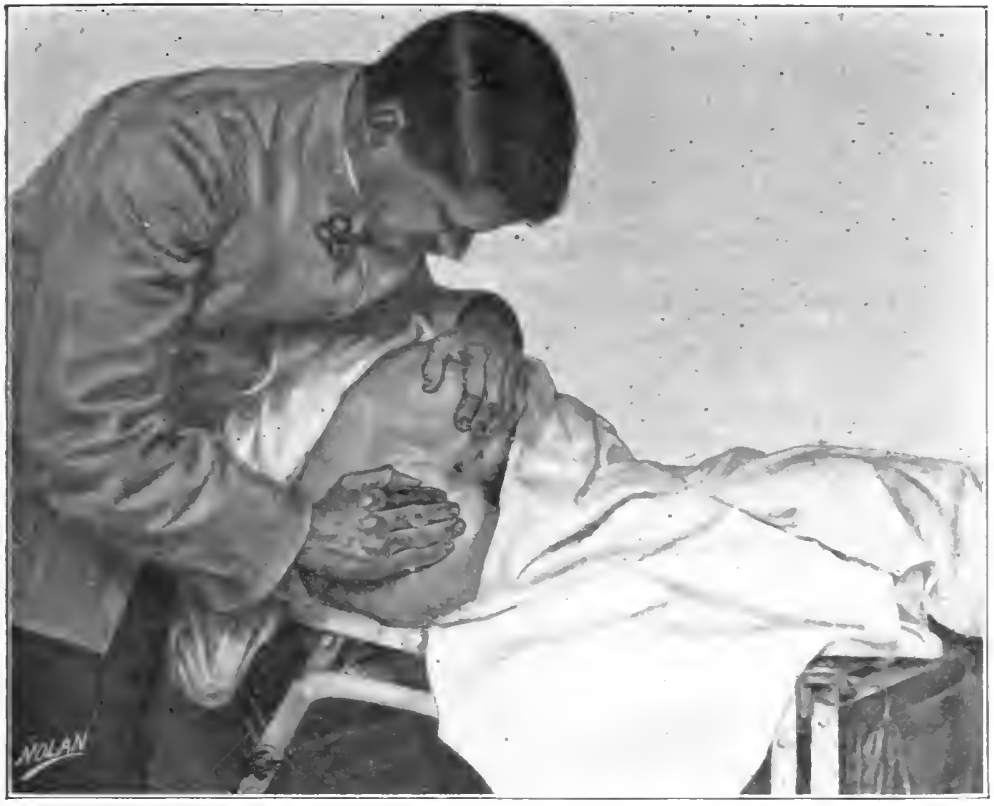

Fig. 12.

\section{External Inspection.}

This drawing well illustrates the posture of both examiner and patient, and shows the extent to which the anus may be dilated by traction of the skin of the buttocks.

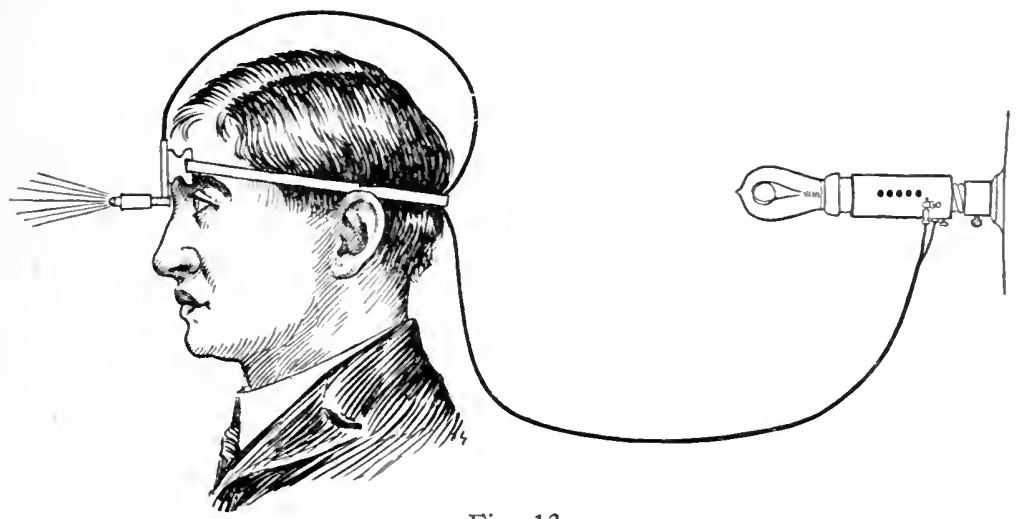

Fig. 13.

Electric Magnifying Headlight.

This is a very simple, inexpensive, and very satisfactory electric headlight. It may be used either on the street current or dry cell battery. It is very light, compact, and can be so adjusted that the light is brought between the operator's eyes. There is a condensing lens blown onto the front of the lamp greatly increasing its efficiency. 
tory. Its germicidal power is equal to that of the bichloride in the strength of $1-2,000$.

Finger cots should always be at hand. The examining finger protected by the finger cot should always be well lubricated before an examination is attempted. There are a number of excellent commercial lubricants on the market, such as Hartz's “Lubra-Septol" and Van Horn's

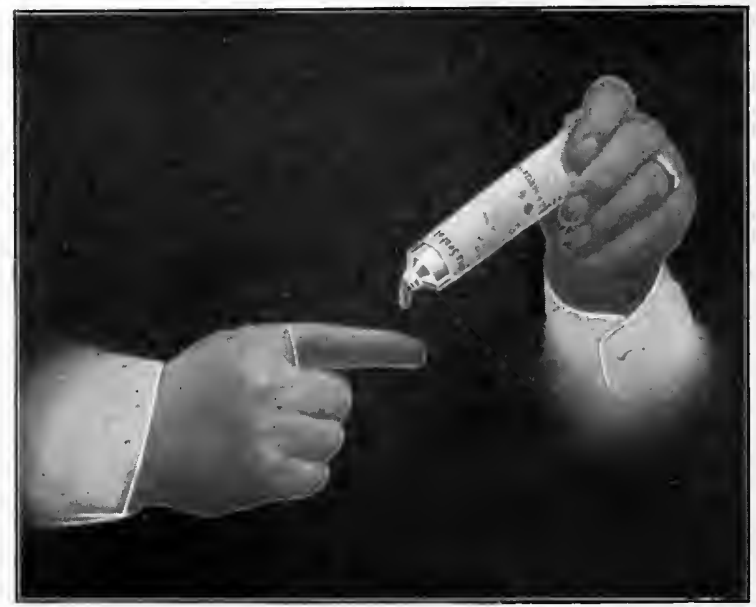

Fig. 14.

Method of Applying Lubricant From Collapsible Tube to Examining Finger Protected With a Rubber Finger Cot.

“ K-Y," but sterile vaseline or oil will be found to answer the purpose equally as well. The author uses a lubricant which has given him perfect satisfaction. It is prepared as follows:

R Oxy-cyanide of Meremry_________- 0.246

Glycerine -

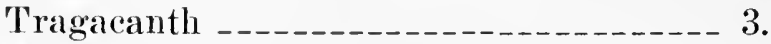

Water .......

Dispense in two ounce collapsible lead tubes. 
The posture of the patient for digital examination is very important. The old method of having a patient simply bend or lean over a chair or table, then inserting the index finger, is not nearly so satisfactory, comfortable, or thorough to either examiner or patient, as the lateral or Sims' position. 'The patient in the Sims' position is relaxed and at ease, and the parts presented in such a manner as to give the clearest view and produce most satisfactory results.

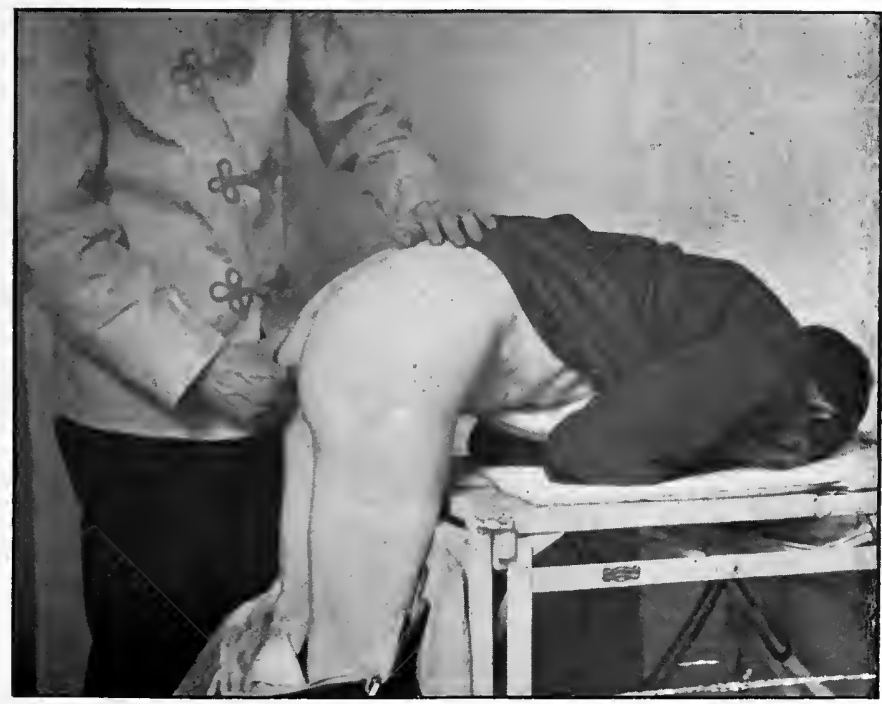

Fig. 15.

Incorrect Method of Digital Examination.

This method was formerly deemed the only proper method of making a digital examination of the anus and rectum. Contrast this with the following illustration.

The wearing of a thin rubber finger cot is done for several reasons. In the first place, it protects the wearer from infection. It also prevents the soiling of the finger with fecal material, pus, or discharge with their dis- 
agreeable odors. It does not interfere with the sense of touch, which can be educated to extreme delicacy even with the cot. From the patient's standpoint it is much more desirable-the smooth rubber covering over the finger enabling it to enter much more easily than the unprotected finger, and there is no danger of irritating sensitive areas with the finger nail. If one wishes to make a digital examination, and a finger cot is not available, the nail of the examining finger should be trimmed close and the crevices under and around it filled by scratching the surface of a bar of soap. The rest of the

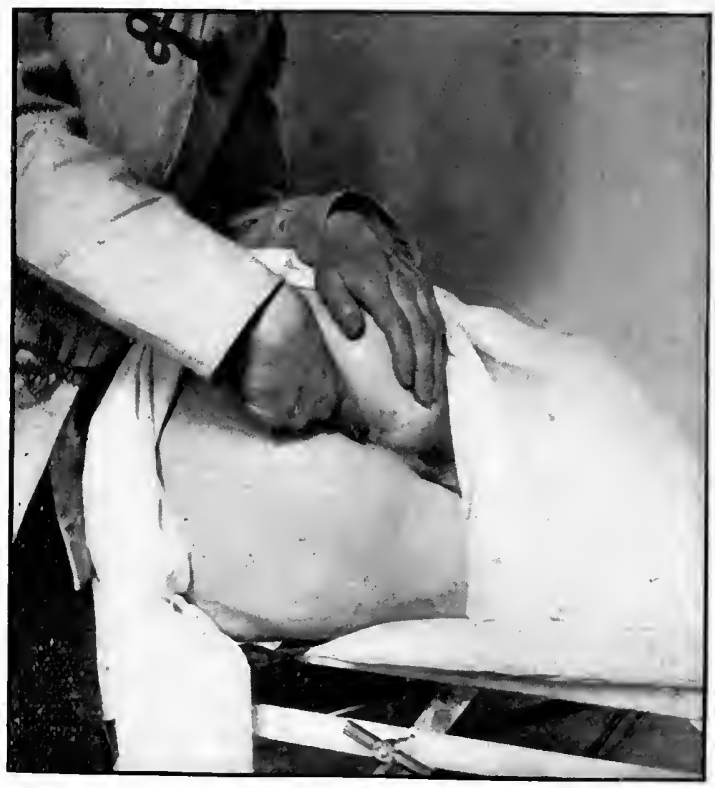

Fig. 16.

Correct Method of Digital Examination.

With the patient in the lateral or Sims position, the examiner standing opposite the sacral region of the patient, digital exploration of the anus and rectum can be accomplished with much more thoroughness, satisfaction and comfort to both. 
finger nail should be covered with soap suds, vaseline, or whatever lubricant is handy. After the examination, the lubricating material should be wiped from the finger with a dry cloth or absorbent cotton before washing the hands.

The position of the patient and the examiner as well is shown in the accompanying illustration. The protected and lubricated finger which is usually the index finger of the right hand, is pressed against the anus with its flexor surface toward the posterior commissure, and the patient is asked to bear down. The finger is first entered pointing anteriorly until the sphincters have been passed, and then passed backward and upward in the posterior direction. As the finger enters, it should be gently turned from side to side sweeping over all the surfaces of the anal canal and lower rectum. Any change from the normal, soft, velvety feeling of this region such as elevations, depressions, or indurations should be carefully noted. The location of the feces is also important, particularly where symptoms of interference with normal defecation are presented. It is therefore important not to give an enema before the first digital examination. Unless one wishes to determine conditions high up in the rectum, or to make a recto-abdominal examination, one should not feel too high in searching for the source of painful rectal symptoms. Most of these diseased conditions will be found within the first two inches from the anal outlet. Often in inserting the finger, the various lesions are pushed up into the rectum giving the impression that they are higher than they actually are. It is with the withdrawal of the finger therefore that more valuable information is often obtained than on its introdnction.

Where the sphincters are so sensitive or tightly con- 
tracted as to prevent digital examination being accomplished without great pain to the patient, dilatation of the sphincters by means of local anesthesia should be resorted to The technique of local anesthesia is fully described in Chapter $\mathrm{XV}$, to which the reader is referred.

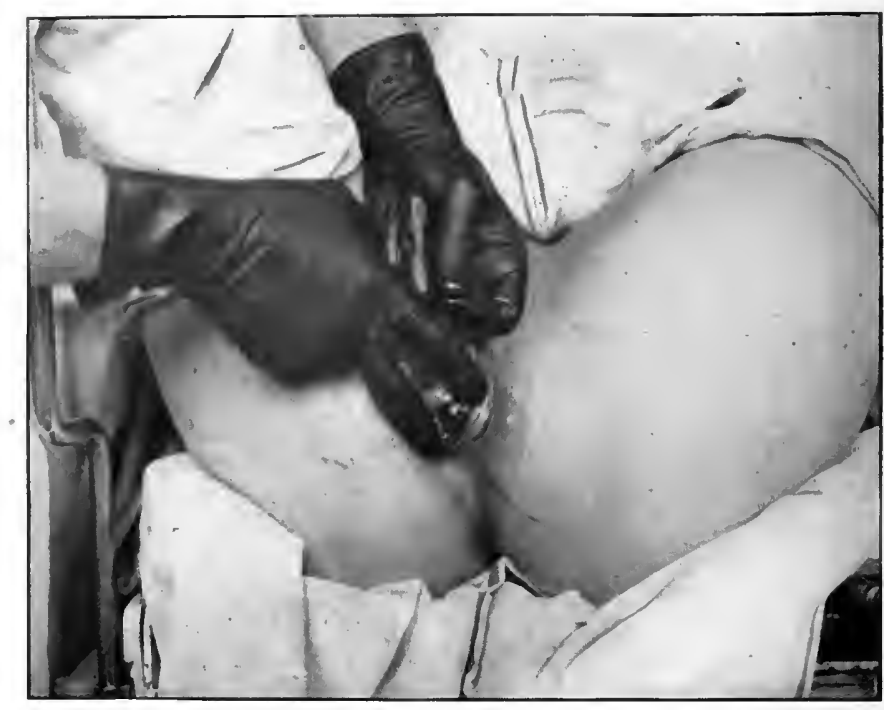

Fig. 17.

Vaginal Eversion of the Anus.

This method is useful in examining the anterior wall of the anus, and lower rectum in female patients; particularly those who have borne children and who have lax perineums.

In women much valuable information can be gained oftentimes by vagino-rectal examination which is accomplished either by the index finger in the rectum and the thumb in the vagina; or by using the index finger of the left hand in the vagina while the right is in the rectum. Often in women where the perinenm is lax, the anus may be evertel by downward and outward pressure of 
the index finger of the right hand in the vagina, while the anus is spread between the index finger and thumb of the left hand.

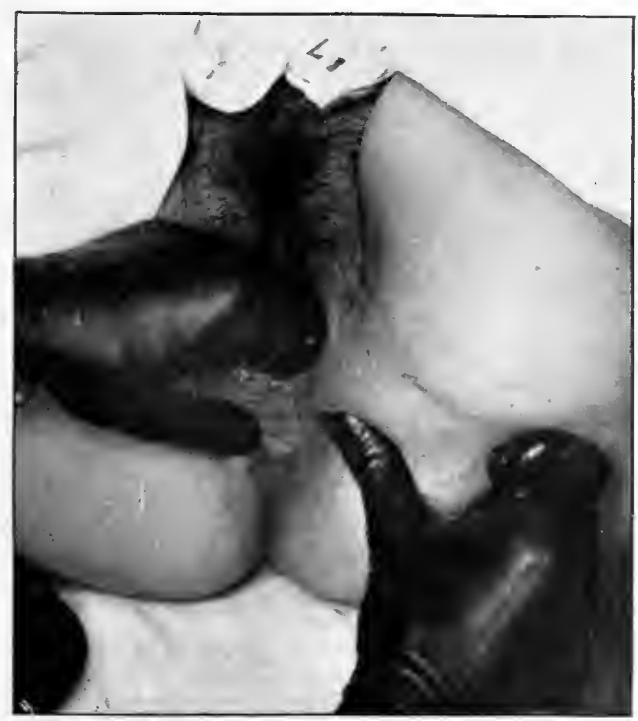

Fig. 18 .

Another Method of Everting the Anal Tissues for Inspection.

-From Crossen's Diagnosis and Treatment of Diseases of Women.

The lithotomy position, while in most cases not nearly so satisfactory for complete ocular inspection of the external parts or the use of the anoscope-nor as comfortable for the patient-has its place in the examination of the patient suffering from ano-rectal diseases. If for some reason or other the patient is not comfortable in the lateral position, which will occasionally be the case in those who suffer from rheumatism or some other joint 
affection; or on account of an unusual amount of adipose tissue the patient's buttocks cannot be well separated in

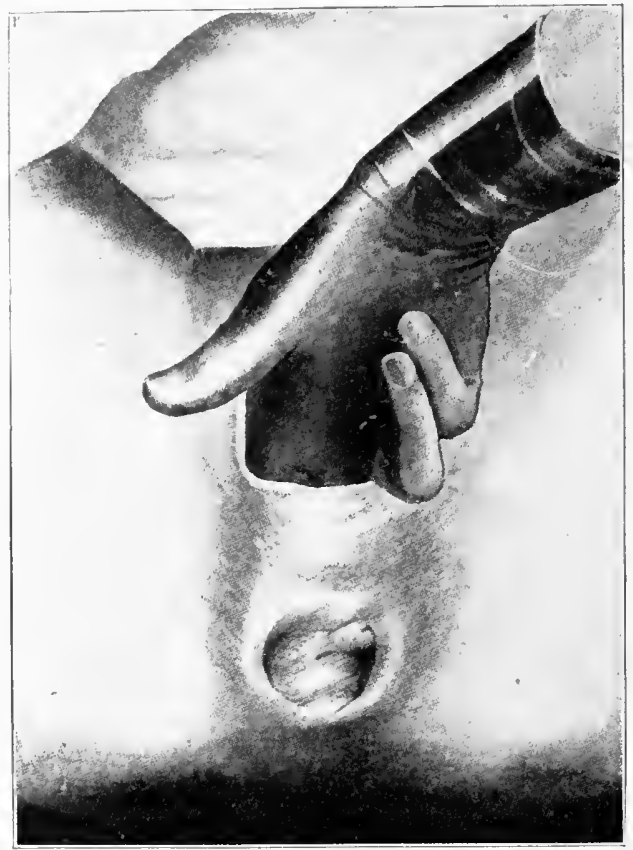

Fig. 19.

Indicating the Amount of Possible Eversion of Anal Tissues When the Pelvic Floor is Lax as in Multiparae.

-Dudley: Practice of Gynecology.

the lateral position, the lithotomy position will be found much more satisfactory. The patient is asked to lie flat upon the table after the elothing has been removed, and a sheet thrown over him. The knees are flexed upon the thighs and the thighs upon the abdomen and the patient's buttocks pulled well down to the edge of the table. The legs are kept in this position either by an assistant or by the use of a Kelly leg holder or Clover's crutch, or by the stirrups or leg holders which accompany the surgical 
table used by the author, known as the Columbus table. In this position the perineal space and the peri-anal ie-

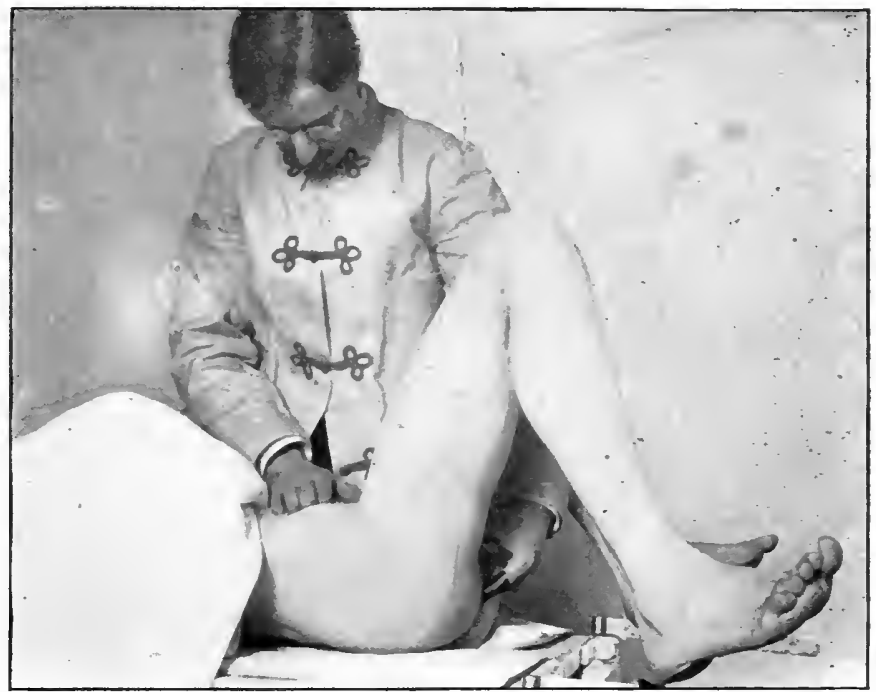

Fig. 20.

Posture and Method of Making Recto-Abdominal Bimanual Examination.

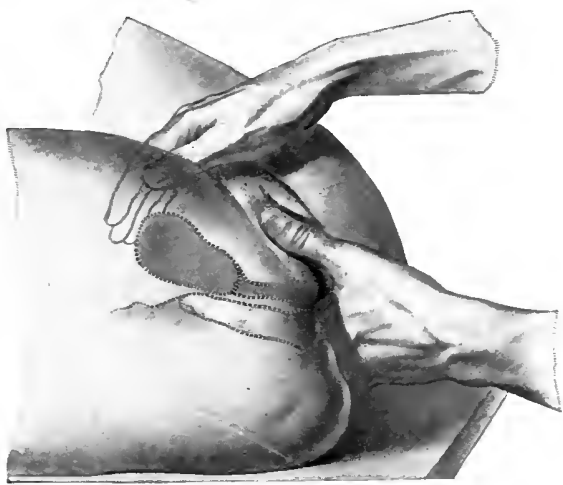

Fig. 21.

Method cf Recto-Abdominal Palpation. The Position of Both Hands in Relation to the Uterus and Vagina is well shown.

Montgomery: Practical Gynecology. 
gion can be inspected and palpated, and in the case of a female patient, examination of the genital organs carried ont at the same time. In this position also the condition of the prostate and seminal vesicles of the male can be made out, and oftentimes the extent and direction of a fistulous tract determined more satisfactorily than in the lateral position. The condition of the coccyx can be determined witl the patient in the lithotomy position by inserting one finger into the rectum and the other hand orer the region of the coccrx, or by the insertion of the forefinger into the rectum and the thumb of the same hand over the location of the coccyx on the outside.

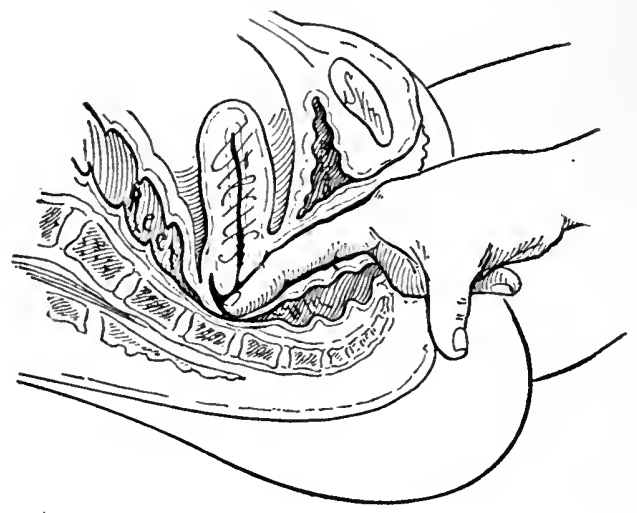

Fig. 22.

Palpation of Rectum Through Posterior Vaginal Wall.

Ashton: Practice of Gynecology.

With the patient in the lithotomy position, bi-manual abdomino-vaginal, and abdomino-rectal examination are accomplished. It is a good, safe plan to include both of these methods in the routine examination of every patient because very frequently unsuspected or beginning dis- 
eased conditions in the pelvis and abdomen are discovered before they have given rise to subjective symptoms. In any case presenting the symptom of sacral backache,

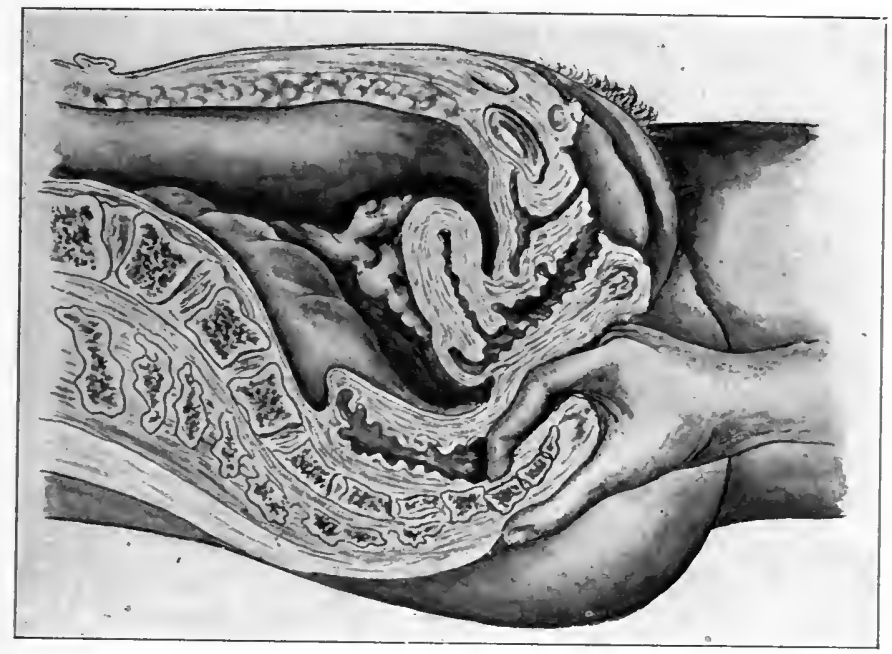

Fig. 23.

Method of Examining the Coccyx With One Hand.

This may also be done with one hand over the region of the coccyx posterior to and above the anus, and the index. finger of the other inside of the rectum.

Hirst: Diseases of Women.

weight in the pelvis, the passage of blood or pus with the stool, or diarrhoea-abdomino-rectal palpation, with the right index finger inserted as high as possible in the rectum, and the left hand over the right and left iliac fossae and above the pubes is imperative.

The squatting position, or the position assumed by the aboriginal races in defecation, is oftentimes of great value in the diagnosis of those conditions made manifest by protrusions from the anal orifice. The patient is asked to 
remove his clothing and to squat as if he wished to defecate. It is best to place a shallow basin or receptacle

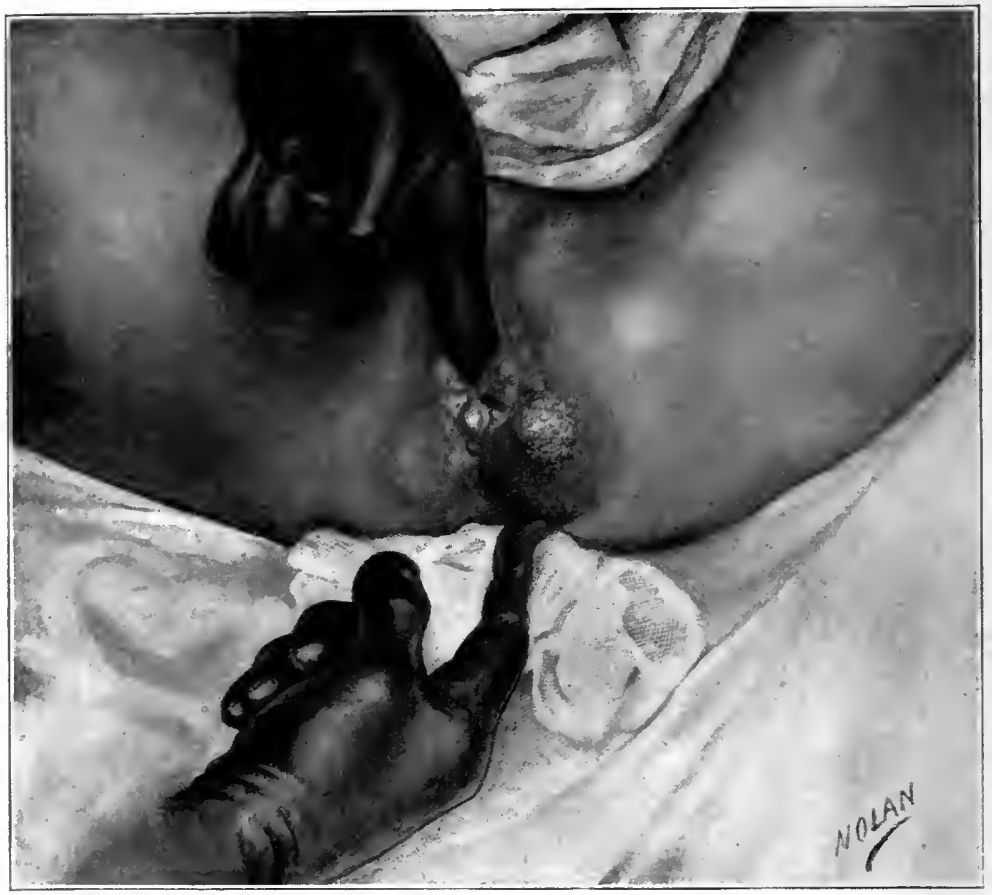

Fig. 24.

\section{Ischio-Rectal Abscess.}

This illustration drawn from a photograph of one of the author's cases, besides showing the point of swelling and fluctuation of the abscess, illustrates the method of binanual palpation in the examination and diagnosis of the condition. At the posterior commissure of the anus will be seen a small external hemorrhoid as well.

underneatl him lest, during his straining efforts. feces, pus, blood or discharge may escape. The patient is then asked to bear down or strain; when in this position, prolapsing internal hemorrhoids, polypi, or prolapse of the 
rectum or anus, will be brought into view in a very satisfactory manner.

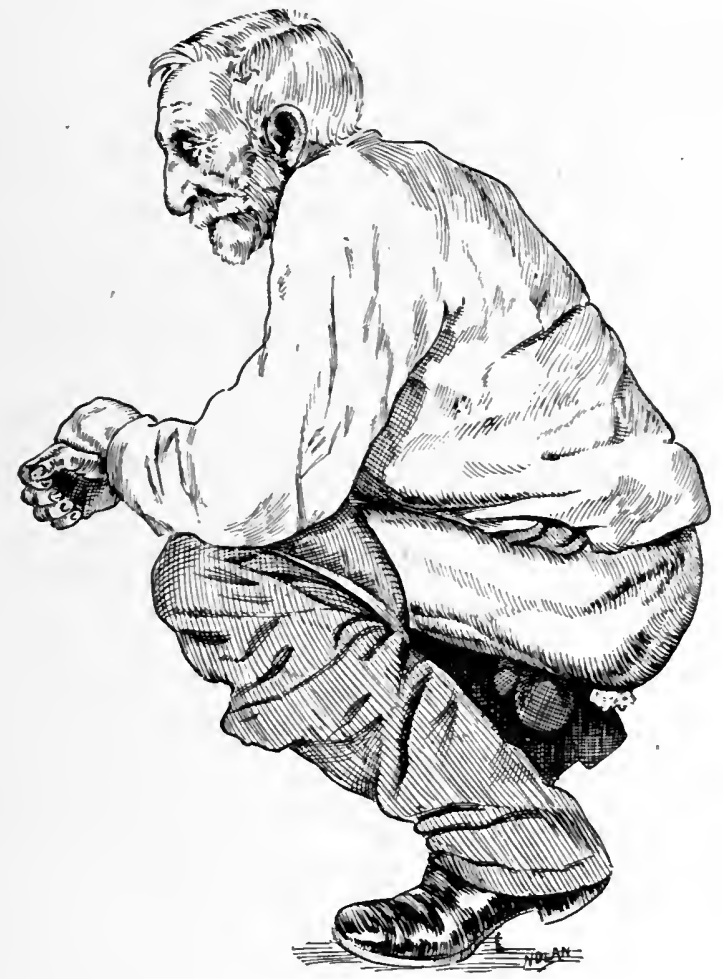

Fig. 25.

Squatting Position.

This position shows the natural posture for defecation, and is useful in extruding prolapsing conditions.

Drawn from a photograph.

Before proceeding to internal inspection, the rectum should be emptied by means of an enema of soap suds and water. If one's office equipment does not include an irrigator, a two quart fountain syringe will answer very nicely. Another very simple method is to use a three or 
four ounce soft tipped, all-rubber bulb sylinge, known as the ear and ulcer syringe. With the patient in the lateral or Sims' position, a pint or more of solution can be gently injected and the rectum cleansed in a very satisfactory manner-the patient being allowed to rise and go to the toilet to expell it.

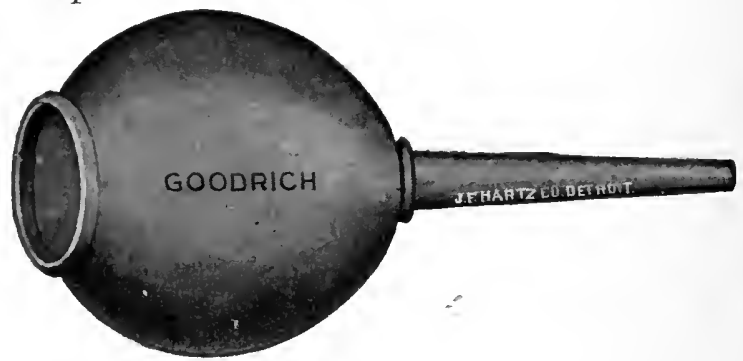

Fig. 26.

Three Ounce All Rubber Bulb Syringe.

Useful in irrigating and in giving enemas when ordinary irrigator is not available.

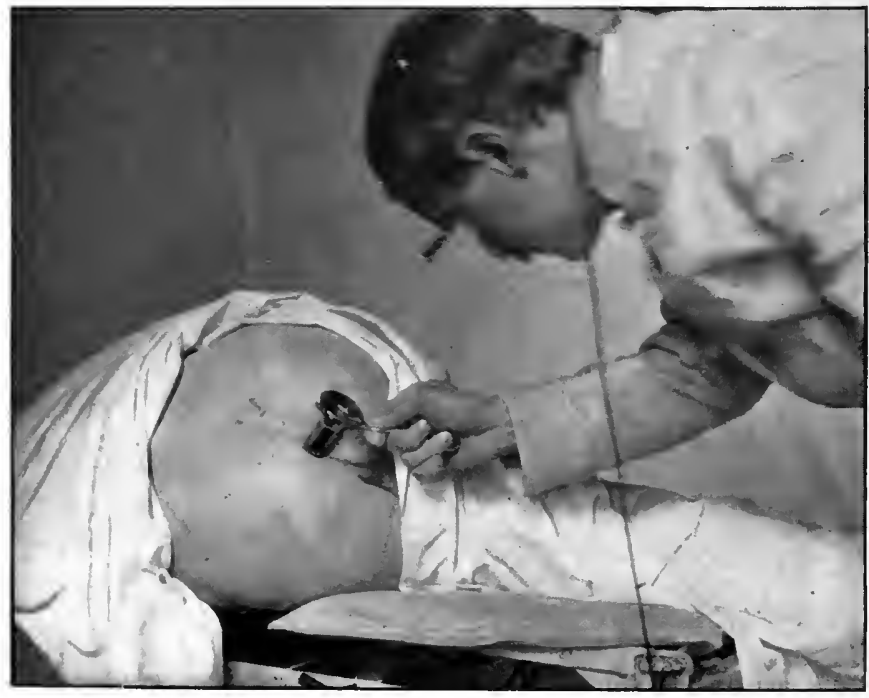

Fig. 27.

Posture and Method of Using the Author's Fenestrated Anoscope, for Examining the Anal Canal. 
Internal inspection of the anus, rectum, and sigmoid is best accomplished with the patient in the knee-shoulder position. The patient who has been lying in the Sims' position; is asked to kneel on the table and to maintain the kneeling position while the examiner brings the left shoulder down to the table flush with the knees. The patient should not be allowed to rest on the elbows as the trunk must present enough of an inclined plane to allow atmospheric dilatation of the rectum, when the examining instruments are inserted, and allow the other abdominal organs to fall away from the rectum. The accompanying illustrations clearly show the difference between the correct and incorrect postures.

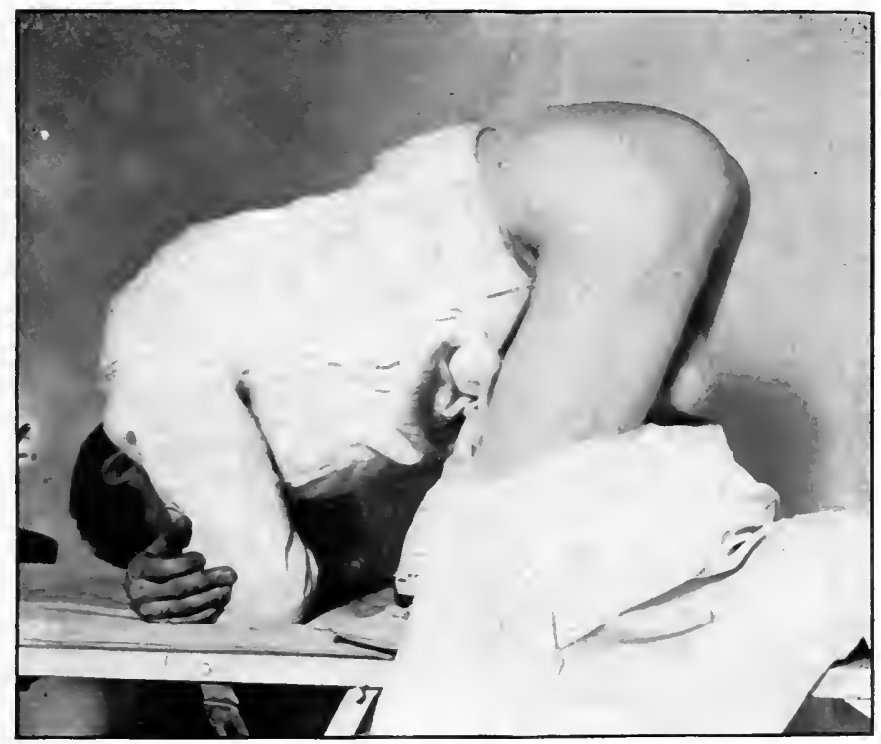

Fig. 28.

Knee-Elbow Position.

This position is often mistakenly employed in proctoscopy, and should not be confused with the knee-shoulder position, as depicted in the following illustration. 
Oftentimes the internal opening of a fistula can be determined by the injection through its external opening of a solution of 25 per cent peroxide of hydrogen. Upon examining through the proctoscope, while injecting, the intermal opening can be easily located by the appearance of the bubbling peroxide solution. Solutions of methylene blue or milk of magnesia or bismuth can also be used in like manner for a similar purpose.

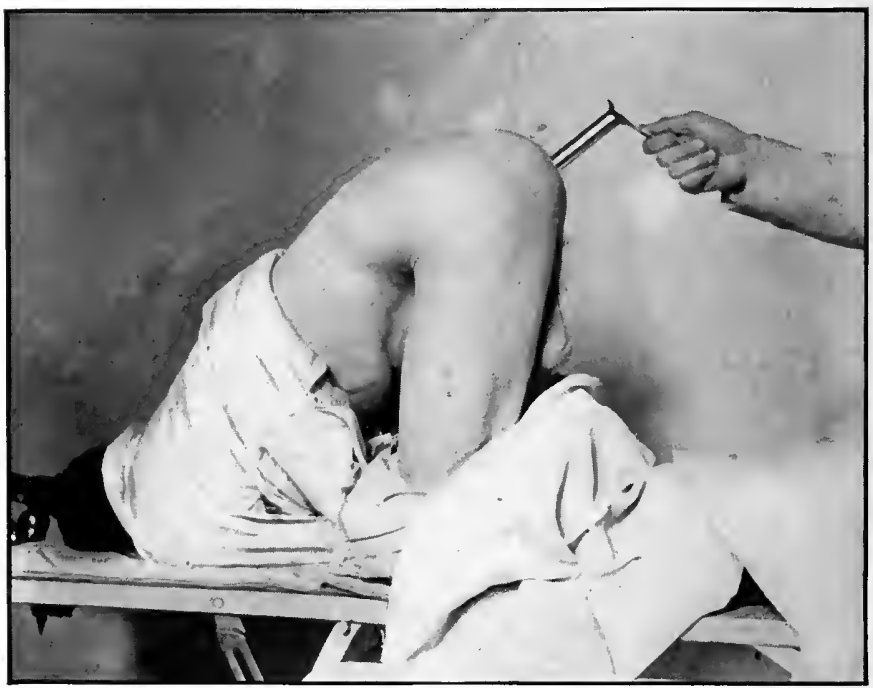

Fig. 29.

\section{Knee-Shoulder Position.}

This is the correct posture for proctoscopic examination. By comparing this with the preceding one, it will be seen that in the kneeshoulder position much more of an inclined plane is produced. Note the direction in which the proctoscope is entered.

For internal inspection of the anal canal, the lateral Sims' position is sufficient.

Instruments.--The instruments required for inspection of the anal canal or anoscopy are: a cylindrical ano- 
scope whose internal opening is cut on the slant and containing an obturator tapering to a blunt round extremity (see figure 30); the tapering, adjustable fenestrated anoscope with closed extremity (see figure 31 ); a fine probe (see figure 32 ), made of pure silver, and a pair of dressing forceps (see figure 33). An ordinary Kelly anoscope (see figure 34 ) is also oftentimes very useful.

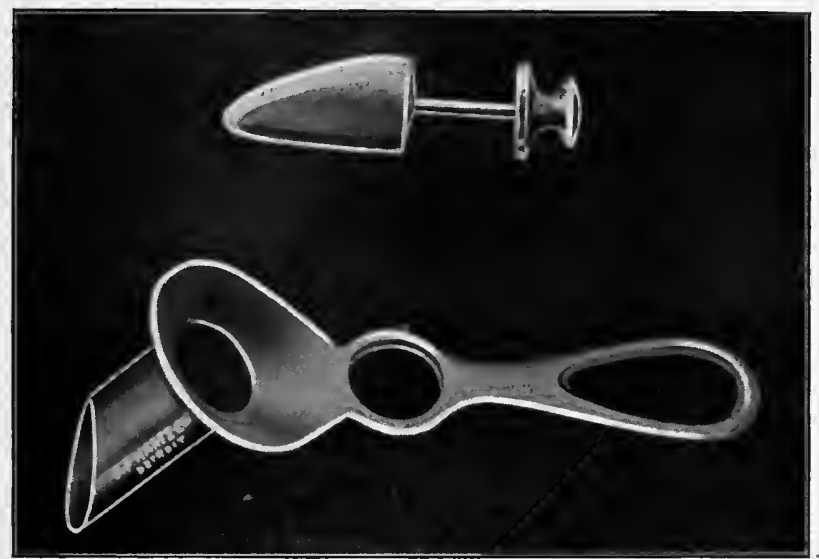

Fig. 30.

Author's Anoscope With Oblique Opening and Slanting Obturator.

Bearing in mind from the digital examination, the location of the lesions in the anal canal, the fenestrated anoscope well lubricated with the opening turned so as to be opposite the lesion when entered, is pressed against the anus and gently inserted while the patient is bearing down against it. If an opening is detected, this may be explored with the soft silver probe, which may be bent easily at any angle, care being taken to use no force and to handle it with extreme gentleness and delicacy. In some cases, the instrument with the opening on the slant 
is used in preference, its opening giving nearly twice the field of the ordinary round opening of the Kelly instru-

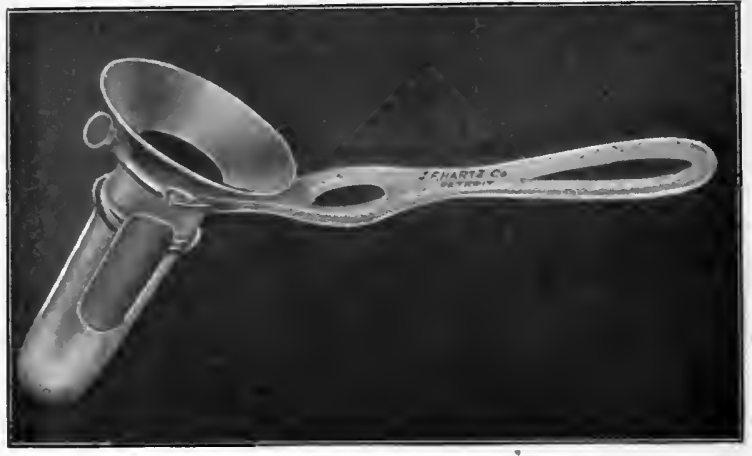

Fig. 31 .

Author's Fenestrated Adjustable Anoscope.

This instrument is provided with a closed extremity; has a fenestrum $13 / 8$ in. long by $1 / 2$ in. wide; can be revolved so that the fenestrum can be placed at any angle in relation to the handle, and on account of the peculiar shape of the ferrule at the proximal end of the fenestrum, is self-retaining.

ment. The Kelly anoscope, however, is useful in exposing conditions which prolapse-the patient being asked

Fig. 32 .

Silver Probe.

This type of probe, equipped with a proper handle and made of pure annealed silver, is the best for use in rectal examination. It is made in many sizes.

to strain and bear down while the instrument is being withdrawn.

By so doing, prolapsing hemorrhoids, prolapsus-ani, or recti, polypi, or papillae are brought out into view. If the view is obscured, at any time, a bit of cotton should be taken up with the dressing forceps to cleanse the parts. 
The knee-shoulder position is by far the most satisfactory in the author's experience for examination of the

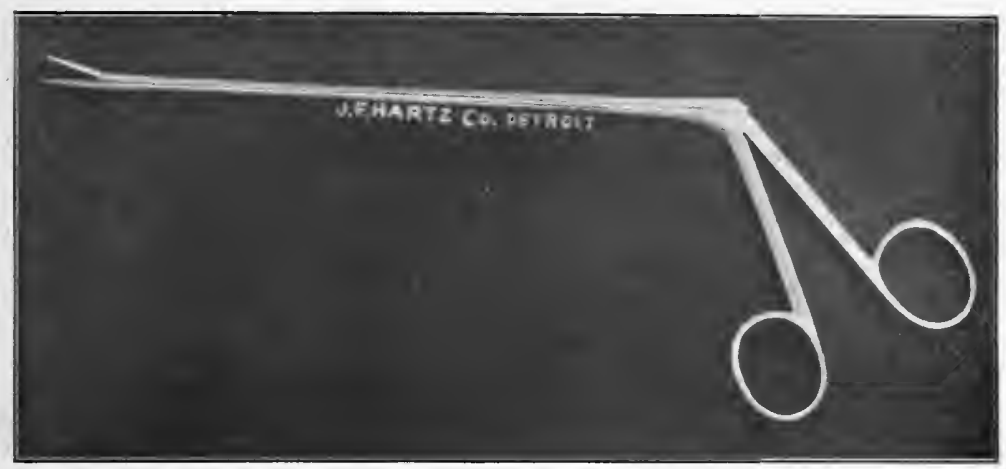

Fig. 33.

Long Alligator Forceps.

These are made in different sizes raiging from 9 to 14 inches in length and are very useful in proctoscopic and sigmoidoscopic examination.

rectal cavity and most of the sigmoid. Not only does the atmospheric pressure balloon out the rectum, to its

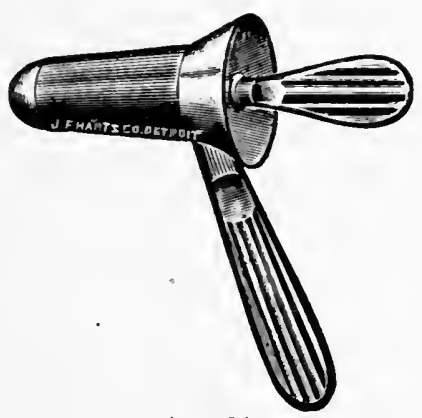

Fig. 34.

Kelly Anoscope.

Useful in prolapsing conditions.

fullest capacity, but this position also removes the pres. sure of other abdominal organs from the rectum by allowing them to fall away. 
'The only instruments required for proctoscopy or ocular inspection of the rectal cavity are, a cylindrical proctoscope, from four to six inches in length and from threequarters to seven-eighths of an inch in diameter, and a

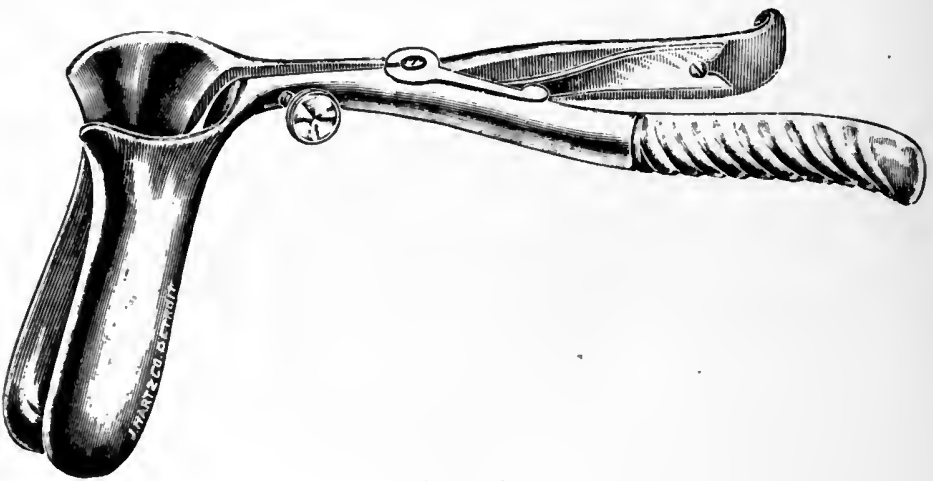

Fig. 35.

Bivalve Rectal Speculum.

This is an instrument formerly used for rectal examinations but which, in the author's opinion, has absolutely no place in modern methods of cxamination. It should be used in operative work, and only when the patient is under general anesthesia.

pair of long alligator forceps. In an emergency, a very fair inspection of the rectal cavity may be had without any instruments whatever. The technique of proctoscopy without instruments, is as follows: With the patient in the knee-shoulder position, the index finger of the right hand, protected by a finger cot, and well lubricated, is gently inserted and the sphincter massaged; then the index finger of the left hand, similarly protected and lubricated, is introduced back to back with the finger in the rectum. The introduction of the second finger should be done slowly and gently and with a massage motion. When it has been introduced to an equal depth with its fellow, that is, up to the second joint of the finger, the 
fingers should be gently separated. The atmospheric air then rushes in with an audible hiss, and the rectum balloons out so that it ean be examined with the aid of the electric headlight or reflected light from the head mirror.

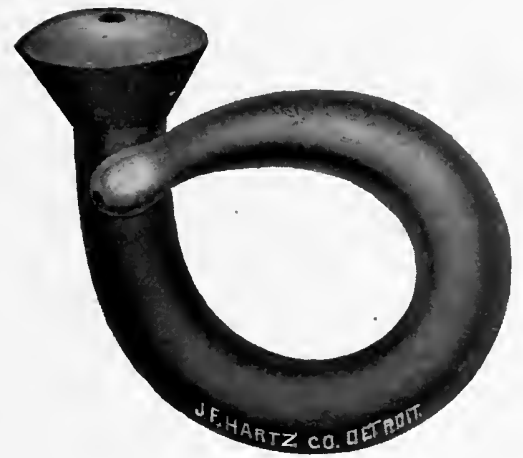

Fig. 36.

\section{Wales Rectal Bougie.}

This is made of flexible rubber and provided with a canal through which irrigation may be given and which allows the entrance of atmospheric air and escape of gas during its introduction. There are twelve different sizes.

With this method, however, one cannot see behind the rectal valves or folds of Houston, and it is only of value where a suitable examining instrument is not at hand and the lowermost portion of the rectal cavity only is to be explored.

The technique of proctoscopy is as follows: With a proctoscope whose outside diameter does not exceed the diameter of the operator's index finger, all parts of the rectal cavity can be successfully explored, and its introduction causes no more pain or discomfort than digital examination. The instrument used by the author is a modification of that devised by T. C. Martin. It is five and one-half inches long from the edge of the flange to 
the tip of the obturator. Its outside diameter is threequarters of an inch. It is provided with an obturator made of metal, with a conical extremity which fits it very snugly. The obturator is channeled so as to allow the ingress of air during its introduction. With the patient in the knee-shoulder position, the well lubricated proctoscope is pressed against the anus, pointing in

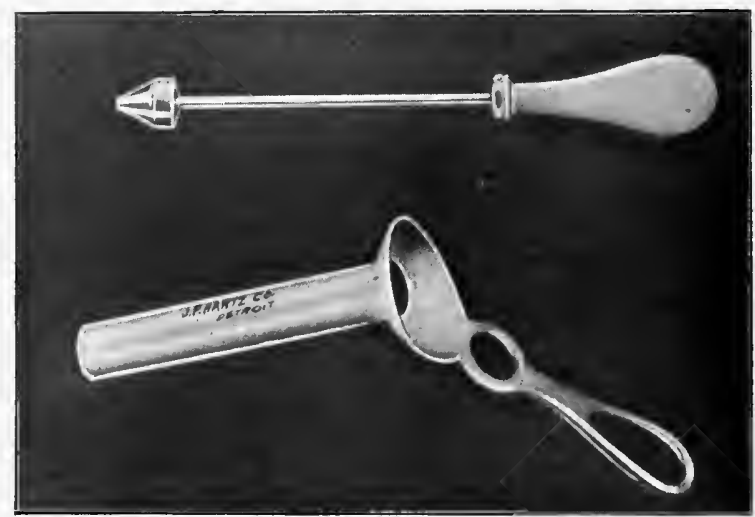

Fig. 37.

Author's Modification of the Martin Proctoscope.

Provided with a metal obturator with conical extremity, which contains an air vent running through its entire length. It is $5 / 8$ of an inch in diameter and six inches long.

the direction of the patient's umbilicus, and the patient asked to bear down, as in the act of defecation. While he is doing so, the proctoscope is inserted gently, first downward and forward, until the anal canal has been passed; when it is tilted upward and backward and the rectal cavity is entered without difficulty. By asking the patient to bear down during the introduction of the instrument, the patient forces his anus down over the proctoscope, as it were, and introduction is accomplished 
with much ease. Holding the proctoscope in the left hand, the obturator is withdrawn with the right. Inspection of the entire rectal eavity can then be accomplished with as much ease and completeness as the examination of the nose or throat. The proctoscope should always be entered to its fullest length before the obturator is withdrawn.

After having examined the uppermost part of the rectum, noting the appearance and condition of the rectosigmoidal juncture, it is slowly withdrawn, the exanniner in the meanwhile noting the condition of the lining membrane of the rectum, the rectal valves, and anal canal until the instrument is completely withdrawn. If, upon the withdrawal of the obturator the opening of the proctoscope seems closed by a wall of rectal mucous membrame, by manipulating the instrument so that its inner extremity is moved to one side or the other, the obstruction will often be found to be one of the rectal valves, or folds of Houston; and on pushing this to one side with the instrument, a new field is exposed to view. With the proctoscope in position, the size, density and thickness of the rectal valves can be noted by means of a probe or applicator bent at a right angle; nlcerations of the rectal wall, their extent and severity noted; the condition of the circulation of the rectum; the presence of polypi-in fact, any deviation from the normal smooth pinkish red appearance of the mucous membrane of the normal rectum easily made out by this method of examination. While the proctoscope is in position, local applications to diseased areas, sprays, insufflations and other therapeutic measures, when indicated, may be carried on under the direct guidance of the eye. The alligator for- 
ceps are useful for swabloing out the rectum and obtaining tissue for microscopical examination.

The exaggerated lithotomy position also sometimes known as the genito-urinary position, is very useful when it is necessary to examine the sigmoid flexure. This position is secured by putting the patient into the lithoomy position as above described, and then slowly lower-

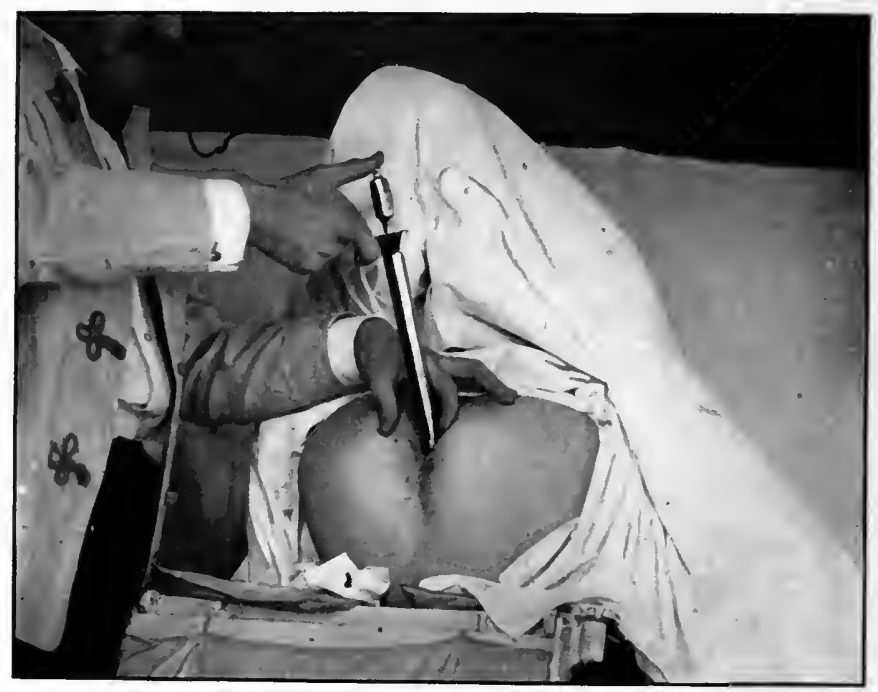

Fig. 38 .

\section{Exaggerated Lithotomy Position.}

. Illustrating posture of the patient and technique of the introduction of the sigmoidoscope.

ing the head of the table so as to leave the buttocks somewhat ligher than the patient's shoulders. 'This puts the patient into a sort of semi-Trendelenburg position with the thighs and knees flexed. In this position it will be found comparatively easy to introduce the sigmoido- 
scope and secure atmosplieric dilatation of the sigmoid flexure.

The instruments necessary for the ocular inspection of the sigmoid flexure or sigmoidoscopy, are sigmoidoscopes varying in length from nine to fourteen inches, and from three-quarter's to an inch in circumference, and

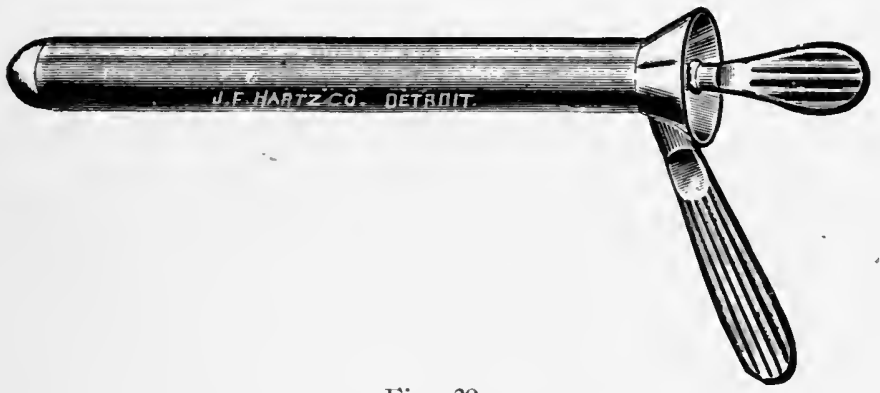

Fig. 39.

Kelly Sigmoidoscope.

This is made in sizes varying from eight to fourteen inches in length.

the long alligator forceps. The instrument devised by Kelly is very serviceable, but its introduction has been

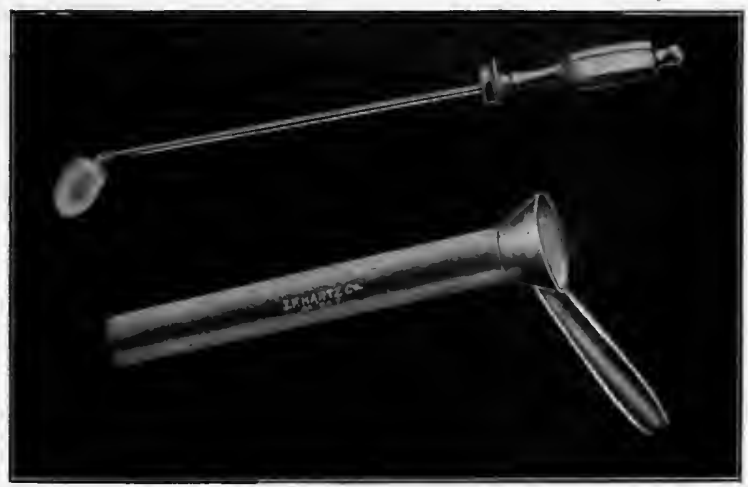

Fig. 40.

Sigmoidoscope Provided With the Author's Tilting Obturator.

The tilting obturator is of value in the insertion of the sigmoidoscope, allowing it to round the sacral curve with greater facility. 
made much easier by the use of an obturator whose projecting extremity tilts so as to allow of easier introduction in rounding the curve of the sacrum. Tuttle has devised such an instrument as has also the author. The only other instrument required is a long alligator for-

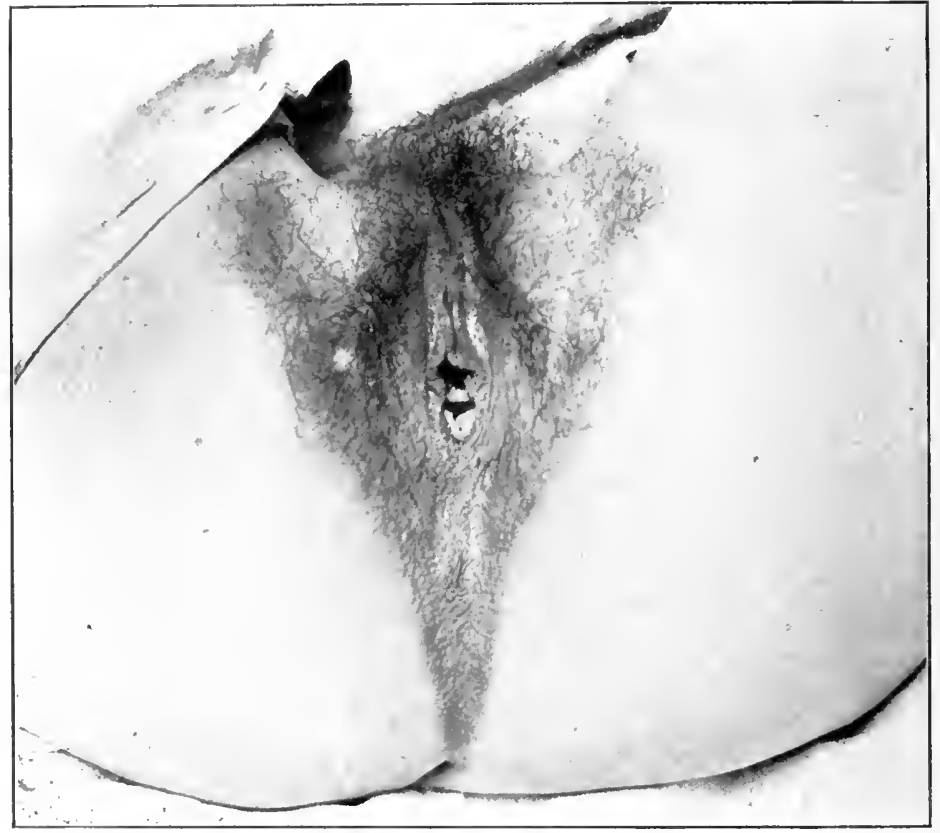

Fig. 41.

\section{Atresia Ani Vaginalis (Complete.)}

Photograph of author's case. 'This illustrates a case of complete absence of the anus with the rectum emptying itself through the vagina. This patient was 25 years old and did not know until shortly before consulting the author, that she was different from other people. She had partial control of her fecal movements by an over-development of her sphincter-vaginae. At the normal location of the anus was found a rudimentary external sphincter. The case was operated upon by the author, the vaginal opening closed and the rectum brought down to the normal anal site, with the result that the patient has an apparently normal anus with good control. The above photograph well shows the scptinm separating the rectal opening of the vagina from the upper vaginal canal. 
ceps for use in swabbing out the sigmoid cavity and for the purpose of removing tissue for microscopical examination. Sigmoidoscopy may be accomplished with the patient in the knee-shoulder position, but much more satisfactory results are obtained from the employment of the exaggerated lithotomy position.

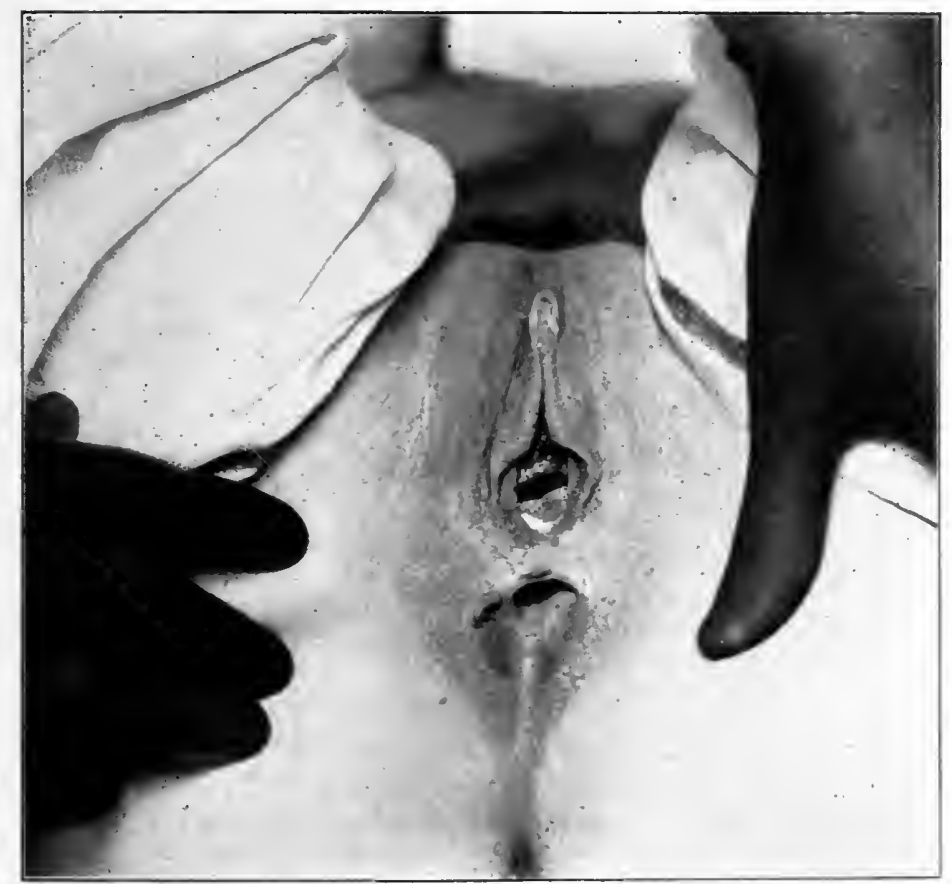

Fig. 42.

\section{Atresia Ani Vaginalis (Incomplete.)}

This photograph, taken from one of the author's cases, seen in consultation, differs from the preceding in that while the patient passed her stools through the vaginal opening, the anus was not entirely occluded, there being a small ano-vaginal fistula. This patient was 23 years of age and had a remarkably well developed sphincter vaginae and was able to well control her fecal movements through the vulvar orifice. This case was likewise operated and the rectum restored to its normal position with a good functional and cosmetic result. The external sphincter muscle was more fully developed in this case than in the preceding one, and control followed much more rapidly. 
Before leaving the subject of examination of the patient, the anthor would advise his readers to carefully examine every patient to make sure that there is not present some congenital defect or malformation of the anus or rectum. Every infant at birth should be examined by the attending obstetrician to make sure that the ano-rectal canal is patent, as imperforate anus, while said to occur but once in 10,000 eases, seems to the anthor, in his own experience and that of his professional friends with whom he has consulted, to have occurred apparently more frequently. If imperforate anus is not recognized, the child will either die in a few hours or days if the condition is not remedied, and even then the operation is attended with a very high mortality; or nature will occasionally form a new outlet for the escape of the feces. In girls this happens more frequently through the vagina and in male infants through the scrotum, bladder or mrethra. 'Two cases have come under the author's notice (See Figs. 41 and 42), in which young women were allowed to grow to the ages of 23 and 25 respectively with congenital defects so serious as to preclude the possibility of marriage until remedied. In one (Fig. 41) there was a complete absence of an anal orifice, and in the other (Fig. 42), an aperture about onefiftl of the normal size. In both cases, defecation took place through the false opening into the vagina. 


\section{CHAPTER IV.}

\section{CONSTIPATION AND OBSTIPATION.}

Constipation may be defined as the voiding of insufficient amounts or the abnormally prolonged retention of fecal material in the intestinal canal. Constipation in contradistinction to obstipation, is due to purely functional diseases or conditions of some portion of the intestinal tract. Obstipation, on the other hand, is a condition in which there is a sufficient quantity of fecal material, and a normal functional activity; but in which some deformity, growth, flexion, constricture, or foreign body in the intestinal canal offers a mechanical obstruction to the passage of the fecal current. These two conditions are so frequently confounded in the mind of the average practitioner that the distinction must be alwars born in mind; for the treatment of these conditions, while they may present similar symptoms, is entirely different.

Constipation is really but a relative condition. One individual may have two or three passages daily and still be constipated, while another individual may have but one passage a week and not be constipated.

Constipation in itself is not a disease but merely a symptom of a great many diseased conditions, but is so often the only apparent symptom of which the patient complains, that its discussion as a separate disease entity is deemed advisable. 
Obstipation is caused by such mechanical conditions as malformations of the intestinal canal, stricture, adhesions, pressure from the pregnant nterus and the various abdominal tumors, angulation, enteroptosis, stenosis of the ileo-cecal valve, fecal impaction, foreign bodies, hypertrophied rectal valves, prolapsus, large hemorrhoids, enlarged prostate and hypertrophied sphincters.

Chronic constipation is a condition which affects a large proportion of all the patients treated by every practitioner of medicine. " It is a condition which is brought about by our modern, so-called "strenuous life." We find it in the infant and in the nonagenarian. It is due to a great many factors, and in order that one may understand it more fully, I will review some points in the physiology of peristalsis and defecation.

Physiology of Defecation. Up to the last moment at which the fecal mass is expelled from the anus, the ingested materials are carried through the intestinal tract by what is known as peristaltic action. After the food has entered the stomach and the albuminoids converted into peptones, it passes through the pylorus into the small intestine. As the stomach contents pass through the pylorus, they are acid. The secretions in the small bowel_the bile and the pancreatic juice, being alkaline; when the acid stomach contents are poured into the small intestine, coming in contact with the alkaline intestinal secretions, a stimulation, or irritation is caused, which produces a wave of muscular contraction, or peristalsis.

At the same time that the chemical reaction of the acid stomach contents upon the alkaline contents of the intestine is going on, certain gases are created. These gases are not abnormal but serve a most useful purpose. It is 
when they are in too great quantities and too severe peristalsis and consequent too great distension of the intestinal canal ensue; that they are harmful. They then cause atony or paralysis of the circular muscle fibres and loss of tone. These gases are largely reabsorbed by the blood vessels or discharged with the feces. If these gases in their downward passage meet any obstruction, they are forced backward into the stomach and are discharged in this direction.

Another important somrce of stimulation to the coats of the bowel is the harsh, indigestible particles of food which are not acted upon by the digestive secretions. These also irritate, and stimulate the contraction of the circular muscular fibres in the small intestine. Of no small importance is the stimulus caused by the to and fro movement imparted to the bowel by the action of respiration. The excursions of the diaphragm upward and downward impart to the small bowel in particular, and also to the transverse colon, a movement which stirs up and churns, as it were, the intestinal contents. It changes the position of the bowel and helps to keep the intestinal contents on the move. It can be easily seen how anything which constricts and prevents the full expansion of the chest will interfere with the intestinal functions and assist in causing constipation.

The intestinal contents are fluid until they reach the ileo-cecal valve. In the cecum they become less fluid and, having to travel against the force of gravity, their movement in the large bowel is checked. Staying, as they do, in this portion of the bowel for some time, the fluid constituents are gradually absorbed, and the nearer to the sigmoid the feces, the more solid they become. The mucous 
membrane of the colon is thicker and not so sensitive as that of the small bowel and requires more stimulation, consequently the stools are more solid in this portion of the bowel. If however, too much vegetable fibre and indigestible material is present, the colon tends to become over-stimulated, over-distended, and atonic; the fecal mass moves very slowly and chronic constipation, and sometimes fecal impaction results. The fecal material. when it reaches the sigmoid, rests until ready to be passed out through the rectum and anus, as a fecal movement.

Causes. It can readily be seen that anything which interferes with the proper development and exercise of the intestinal muscle layers will interfere with the proper movement of the intestinal contents and with expulsion at the proper time. In the first place, enough fluid must be taken into the system daily to keep the intestinal contents in solution and to properly supply the various organs of the body. People who do not drink enough water suffer from constipation because of the re-absorption of fluids from the intestinal tract and consequent hard and dry stools. People who drink great quantities of water with their meals drown their stomach contents; undigested particles of food are sent through the pylorus with gushes of greatly diluted gastric juice; the feeble acid reaction of this mixture does not cause the proper reaction with the alkaline intestinal contents; the proper amount of gases is not evolved and quantities of irritating food particles are passed down the small bowel. This is another cause of loss of tone.

It is a well known fact that carnivorous animals are constipated while the herbivorous animals have full and 
frequent bowel movements. It therefore behooves us to see that a sufficient quantity of vegetable material which will leave undigested fibre in sufficient and not too great quantities, such as corn, celery, beet tops, lettuce, spinach, water cress, endive, kale, and other greens, is incorporated into our daily regimen. It should also contain a sufficient quantity of mineral salts, which are natural laxatives. It should contain sweets within reasonable limits, because of the gas development which goes with them, and the fact that carbon dioxide gas is one of our best laxatives should not be forgotten. Above all, the food of the individual must not be concentrated; it must give sufficient bulk to the stool so that it will properly fill and distend the gut, give it work to do and produce the proper mechanical stimulus to cause contraction. The value of whole wheat bread and bran lies in the quantity of cellulose in the husk, which is a very important element. People who eat too fast do not properly masticate their food, causing improper digestion with improper bowel contents, and have improper stimuli to peristalsis and consequently improper stools.

Outside of dietetic error, the most important cause of constipation is neglect. The school child hears the call of nature, the fecal mass is ready to be extruded, he is receiving powerful stimuli for the dilatation of the sphincters and the expulsion of his bowel contents; but in our modern schools, the lesson hour is more important than the functions of nature! The child is not allowed to go and relieve himself. He restrains nature's efforts; the desire passes away. The continuance of this performance day after day soon makes the child a chronically constipated child. 
While peristalsis is involuntary, in the vast majority of people the voluntary control over the sphineter is normally sufficient to withstand it. The strong expulsive efforts soon weaken when retarded by a tightly contracted sphineter, and shortly the constipated habit is induced. The young girl in society is taken with a desire to move her bowels and either because the time is not convenient and she restrains nature's efforts, the desire soon passes away and she is constipated; or she may be willing to satisfy nature's desire, but in order to reach the toilet room she must perhaps pass through a erowded room, and false modesty prevents her from allowing her friends to see her go in the direction of the toilet room. A very important provision in architecture of homes and institutions should be the plaeing of the toilet room in such an inconspienous place that a person may reach the same without being subjected to the gaze of others.

The business man, the traveller, the physician, the school teacher, the professional man-all refuse to obey nature's call because the time is not convenient, and thus because we liave not time to move our bowels when they want to be moved, we have become a constipated nation! I think this neglect and indifference is the most important cause of eonstipation.

Another contributing cause to the voluntary repression of defecation is the fact that in institutions, and in large buildings, there are not enough toilet rooms for the number of inmates. Where one has to wait long for his turn, the desire is soon lost.

The shape of the toilet seat and its height from the floor are all of importance in the production of a good stool. The seat should be so made that the person using 
it is in the squatting position with the buttocks well separated so as to allow the free excursion upward and downward of the muscles, which go to form the pelvic floor, and the full action of all the other muscles involved in defecation brought into play. People leading sedentary lives, who do not get sufficient exercise, of course, are constipated, for exercise is one of the important factors in keeping all bodily functions normal. There are many other causes which may contribute to the production of constipation in individual cases, but those mentioned are the most important, and the most common.

Diagnosis and Treatment. When the bowel has become atonic, remedies to restore its tone must be enployed. In the treatment of acute constipation, cathartic drugs, suppositories, enemata, all have their proper place; but the victim of chronic constipation should no more be made a drug fiend than the victim of chronic appendicitis. Instead of causing irritating, irregular erratic and violent peristaltic movements at certain times during the day; and instead of changing from one cathartic to another and increasing the dosage-instead of taking away the work of the bowel by flushing with enemata-we should strive to bring that bowel back to its normal tone by imitating' nature's methods. The only place for a cathartic in the treatment of chronic constipation is at the beginning of the treatment.

When a patient consults you, complaining of infrequent or insufficient bowel movements, the first thing to do is to make a diagnosis between constipation and obstipation. The patient should be examined carefully, his abdomen should be palpated thoroughly; the female pa- 
tient should have a bimanual examination; the male patient should have the genito-urinary organs examined, as many cases of constipation are reflexes from bladder and prostatic conditions. The rectum and sigmoid should be thoroughly explored and a complete proctoscopic examination with the patient in the knee-shoulder position is imperative in every patient complaining of impeded fecal movements. After you have satisfied yourself that you have a case of constipation, and not obstipation, to deal witl, and after carefully questioning your patient as to habits, diet and previous history; the question of treatment presents itself.

Dietetic errors should be corrected and the patient instructed as to the time and the quantity and the kinds of food to take. If he is not able to properly masticate his food, he should consult his dentist. The teeth should be put into perfect shape. He should be instructed to drink from six to eight glasses of water in every twenty-four hour's-the first glass on rising, the last glass on retiring. He should drink plenty of water between meals, but very sparingly with meals. He should be instructed to eat a sufficient amount of vegetable foods and not to remove outside coverings of such fruits as pears, apples and peaches, before eating them. He should be instructed to take out-door exercise: to play tennis; to play golf; to go horse-back riding or bicycle riding; to take long walks. He should take breathing exercises, and should develop his abdominal muscles.

Any local condition, such as hemorrhoids, which of themselves do not cause constipation but are caused by constipation, but by their presence prevent natural movements, should be corrected. Fissures, ulcers, or excoria- 
tions of the anus, should be remedied by surgical means or treated locally. Proctitis should be relieved by the proper sprays and medications applied locally. Run down patients should receive in addition, massage from a properly qualified masseur. The abnormally tight sphincter should be dilated or given vibratory massage, and the atonic lower bowel should be properly massager.

A great many drugless methods of treating constipation have been offered to the medical profession. All kinds of electric and external massage, cannon-balls, gymnastics, vibratory massage, and what not, have all been tried, and while satisfactory results have been obtained from each of them in certain cases, there still seems something to be desired in the successful treatment of chronic constipation.

The direct stimulation of the atonic sigmoid and rectum by means of mechanical dilatation has, up to the jresent time, given the best results. Rubber bags, which have been introduced through the proctoscope into the sigmoid and inflated, have been used by Turck and other's with excellent results. Tamponing the rectum and sigmoid with cotton, wool, or gauze, as advocated by MacMillan, has, by its mechanical irritation of the nucous coats of the bowel and its simulating the normal bowel contents produced satisfactory evacuation. The inconvenience of carrying around a tampon or inflated bag in the rectum or sigmoid for from four to six, or eren twelve hours, has, however, militated somewhat against the more general use of these methods. W. Teachnor, of Columbus, O., has successfully treated a number of cases by simple inflation of the rectum and sigmoid by the en- 
trance of air through the proctoscope, while the patient is in the knee-shoulder position.

Author's Method. The author has devised a very simple pneumatic dilator for accomplishing this distention, and has achieved very happy results from its use. No great originality is claimed for this device. It consists simply of a specially devised rubber bag with a stem. which is slipped over the distal end of a Wales bougie

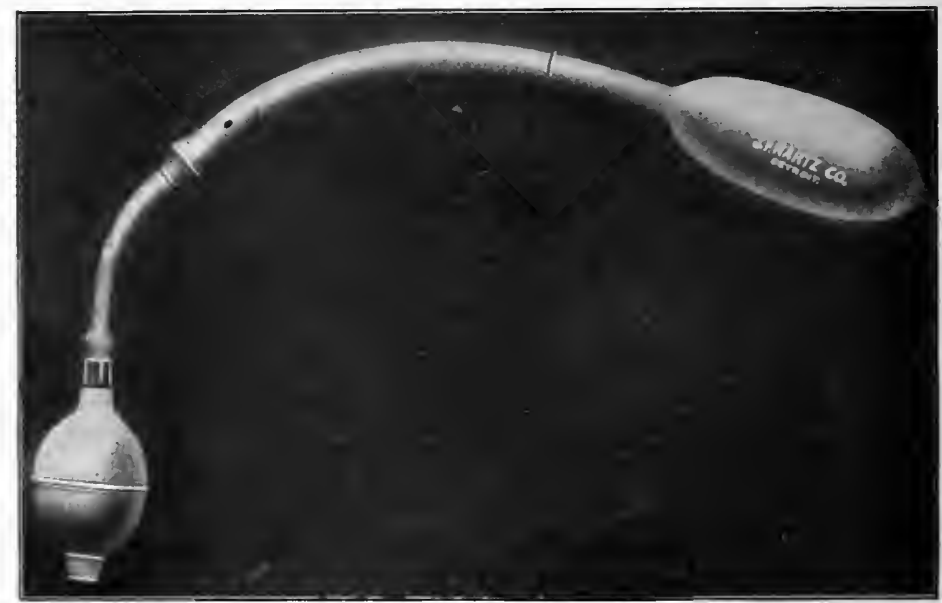

Fig. 43.

The Author's Pneumatic Rubber Dilating Rectal Massage Bag Equipped With a Hand Bulb.

(No. 3-5), the Wales bougie being canaled (Fig. 43) and containing an air vent in the handle which is covered by the finger until the air is to be expelled. Compressed air at a low pressure (one to three pounds) is allowed to slowly enter the bag, and distension to any desired extent is produced. By means of an ordinary cut-off valve this distension can be easily regulated. Where the com- 
pressed air apparatus is not convenient, an ordinary atomizer bulb or a small bicycle pump can be utilized.

The patient is placed in the Sims position. The bag is lubricated and deflated, is twisted around itself as an umbrella is rolled on its handle, and passed upward into the rectum following the backward curve of the sacrum, then into the sigmoid to any desired height. The Wales bougie being firm enough to carry the bag up into the sigmoid, and yet being flexible, does not create any dis-

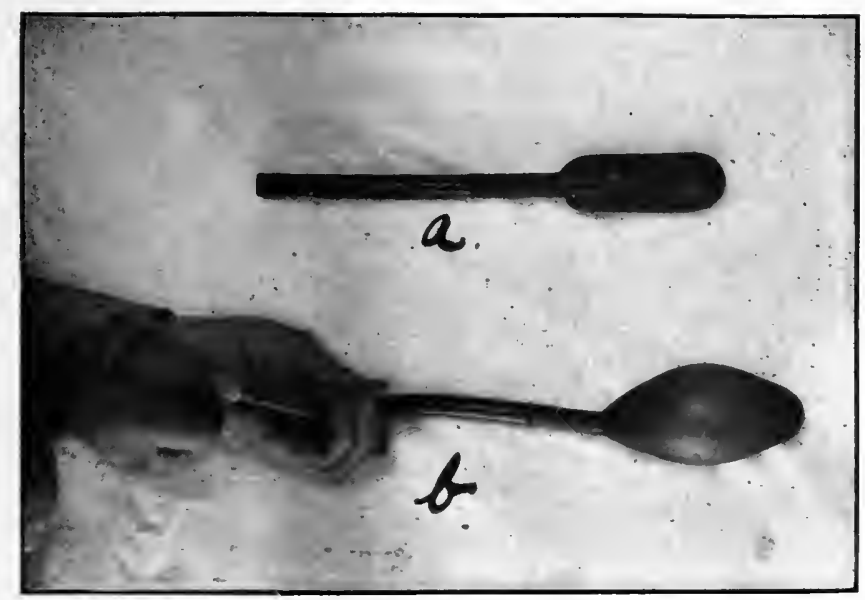

Fig. 44.

Author's Rubber Dilating Rectal Massage Bag.

a. Bag deflated.

b. Showing the amount of inflation necessary in the average case. comfort or do any injury in its passage; and it obviates the use of the proctoscope in its introduction. When the bag is in position, it is slowly inflated until the patient complains of either fullness or slight crampy pain, or a desire to move the bowels. The air is allowed to escape by removing the finger from the air vent in the handle 
of the bougie, and after an interval of five or ten seconds, it is again inflated to the point of tolerance; the cut-off

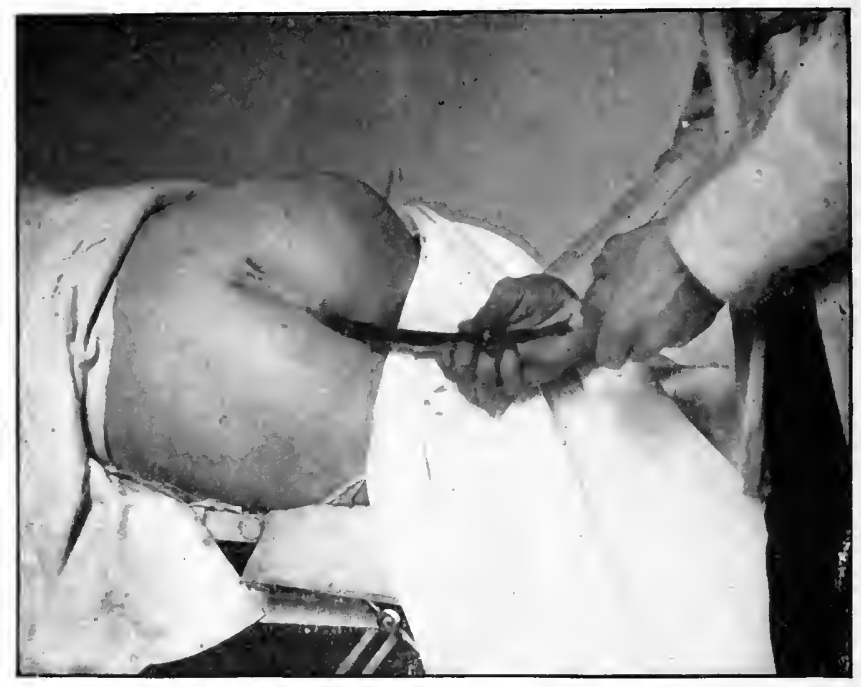

Fig. 45.

Position of Patient and Operator for the Author's Method of Rectal Massage.

This is the best position for both the patient and operator in treating chronic constipation with the author's dilating rectal massage bag.

valve is disengaged and the opening in the bougie is closed with the thmmb, and where the hand-bulb is used, the air vent in handle of the bongie is closed with the finger-tip; and then by a to and fro motion, the apparatms is gently and slowly withdrawn. This method of removing the apparatus is important as it also gently dilates the sphincter muscles.

This treatment is repeated daily for from five days to a week, and usually after the first or second treatment the patient will have a small unaided movement. Cathartics and enemata are, of comse, strictly enjoined. 
Each day the patient will report a slightly larger and more satisfactory defecation. When the defecation approaches the normal, treatments are given only on alternate days. After three or four days, the interval is lengthened to two days, and then to three; and then to four; when the patient is asked to report in five or six days. If he reports satisfactory evacuations daily, he is allowed to go a week, and then, if a similar report is made, he is discharged as cured, but asked to return for another treatment on the first day on which he does not have a normal stool.

If the case is properly diagnosed and instructions as to a regular time for daily evacuations and strict obedience to nature's calls are carried out by the patient, as well as the proper dietary being lived up to; the results from this method of treatment will be very satisfactory, as the experience of several hundred practitioners in all parts of the United States and Canada will testify.

The only internal medication which has been found nec. essary in the author's experience, has been the administration of extract of nux vomica in one-fourth to onehalf grain doses before meals as a tonic to the bowel muscles. Pancreatin in ten grain doses before meals has been found of value in patients who show symp. toms of intestinal indigestion. In those cases where starchy food is found difficult of digestion the administration of Takadiastase in doses of four to ten grains has been found of service. The author has experienced great satisfaction from the administration of white refined petroleum oil, also known as liquid albolene. A prepara- 
tion such as the following is a very satisfactory way of administering it:

Oil of wintergreen or peppermint_---1 part. Refined white petroleum oil__-__-_-_8 parts.

Directions:-A teaspoonful before each meal and at bedtime.

This oil has no value whatever as a cathartic and is not acted upon by any of the digestive secretions. It passes through the stomach and bowel and is expelled unchanged. It acts as a mechanical lubricant to the stool and softens hard masses which have been formed and prevents the formation of others.

Other diseases occurring coincidently with constipation have to be treated according to their special indications and needs.

Obstipation. Obstipation as defined at the beginning of the chapter is a purely mechanical condition, there being some pathological condition which narrows, constricts, kinks, or obstructs the bowel in such a manner as to offer more resistance than normal peristalsis can overcome. Pressure from various abdominal organs, obstruction from intra-abdominal adhesions, torsion, or angulation of the bowel are conditions which can be remedied only by operative interference under general anesthesia and do not come within the scope of this work. Obstipa. tion, however, which is due to hypertrophy of the rectal valves or valves of Houston, fecal impaction, or hypertro. phied sphincters are all amenable to office treatment under local anesthesia. 
The Rectal Valves.-While for several years a great controversy has been raised as to whether the rectal valves of Houston are really valves, or simply constant folds of mucous membrane, nevertheless, the fact that hrpertrophy of these structures does obstruct and impede the flow of the fecal current is now generally admitted. The number of cases reported of obstipation which have been relieved only after section of hypertrophied rectal valves, is now so large that the operation of rectal valvotomy has come to be a recognized form of treatment.

Anatomical studies of the valves in situ and sections of the valve studied microscopically have shown conclusively that they possess all the elements of a typical valve. They are not simple folds of mucous membrane, but are composed of first, mucous membrane; second, a fibrous tissue layer; third, a circular muscular layer; fourth, a longitudinal muscular layer; and, fifth, a sub. serous layer consisting of areolar tissue and fat and containing arteries, veins, nerves, and lymphatics. Under certain conditions these rectal valves become thickened and stiffened by the increased deposition of fibrous tis. sue; in fact, become almost leathery in consistency They may or may not encroach upon the lumen of the borrel. They may not become increased in thickness whatever, but may be simply increased in area so that they occupy from one-half to three-quarters or more of the rectal lumen. Sometimes one valve may be enlarged and sometimes two or three. This form of enlargement presents a firm and unyielding barrier to the normal descent of the feces.

Patients 'with so-called constipation who have run the whole gamut of cathartics, enemas, massage, dietetics, 
electricity, osteopathy and Christian "Science" have not been relieved until they have had a proper proctologic examination and the enlarged rectal valves which were discovered, operated upon. The author has had repeatedly such cases referred to him and the operation of valvotomy has relieved a lange percentage of these cases.

The operation as performed on most of these patients was a modification of that first introduced by 'T. C. Martin, of Cleveland. It was a delicate operation, requiring considerable skill and special apparatus; the results were all that could be wished for. The objections were, first, that without a general anesthetic patients became wearied and restless before the operation was completed; second, the fact that a general anesthetic was required for a number of cases; third, that in cases of unusually large blood vessels in the valve, considerable difficulty was ex-

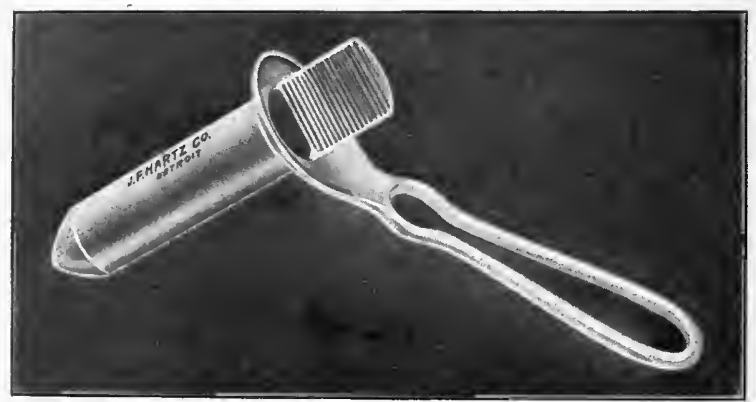

Fig. 46.

Author's Four-Inch Operating Proctoscope.

perienced with hemorrhage; fourth, that the patient was confined to his house or bed or the hospital for from four or five days to a week.

The clamp of Gant and the Pennington clip greatly simplified the operation of valvotomy ; so much so, that it 
could be done in the physican's office without any anesthesia and in a very few minutes. The objection to the use of these mechanical contrivances was the fact of the possibility of their being carried up higher into the bowel after cutting through and also trauma of the rectal mucous membrane caused by the retention and passage of the irregularly shaped, hard, metallic bodies.

Author's Operation for Rectal Valvotomy. The author has devised an extremely simple technique which has proved most satisfactory in his hands, and which by reference to the accompanying illustrations can be readily understood. The patient is put in the knee-shoulder position, and a large operating size proctoscope inserted

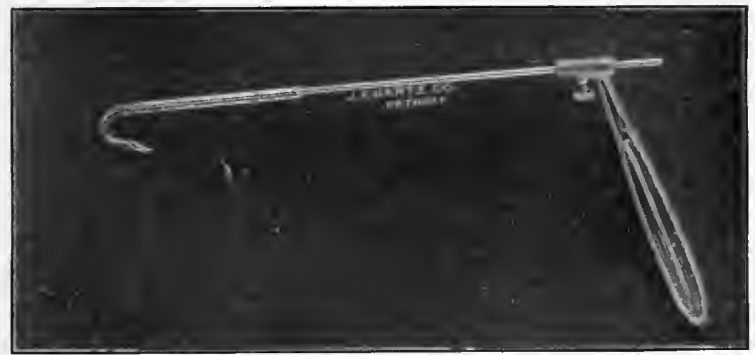

Fig. 47.

Author's Rubber Ligature Carrier or Valvotomy Needle.

after the sphincter has been prepared for it, either by gradual dilatation or by immediate partial divulsion fol. lowing local anesthesia of the parts. 'The anthor's ligature carrier or valvotomy needle depicted above, is threaded through the eye at the curve with a rubber ligature (sizes 5 to 8 , French scale). The ligature passes inside of the curve of the needle and should project about half an inch from the point. The needle, which is nine 
inches long and has a handle bent at an angle so as not to obstruct the view, is then passed up around and hooked

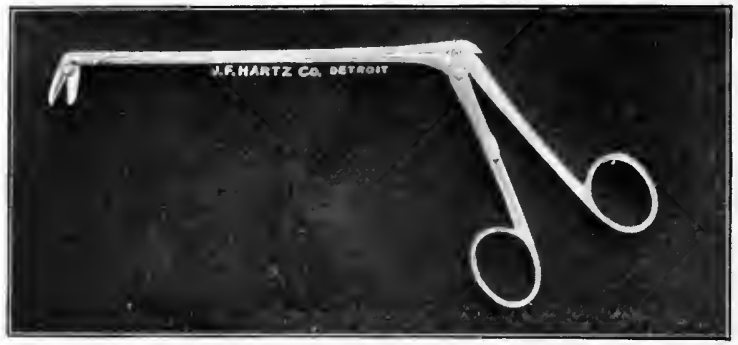

Fig. 48.

Author's Angular Rectal Scissors.

A very useful instrument for any cutting uperation performed through the operating proctoscope.

through the highest offending valve until the point is projected and the ligature can be clearly seen. This end

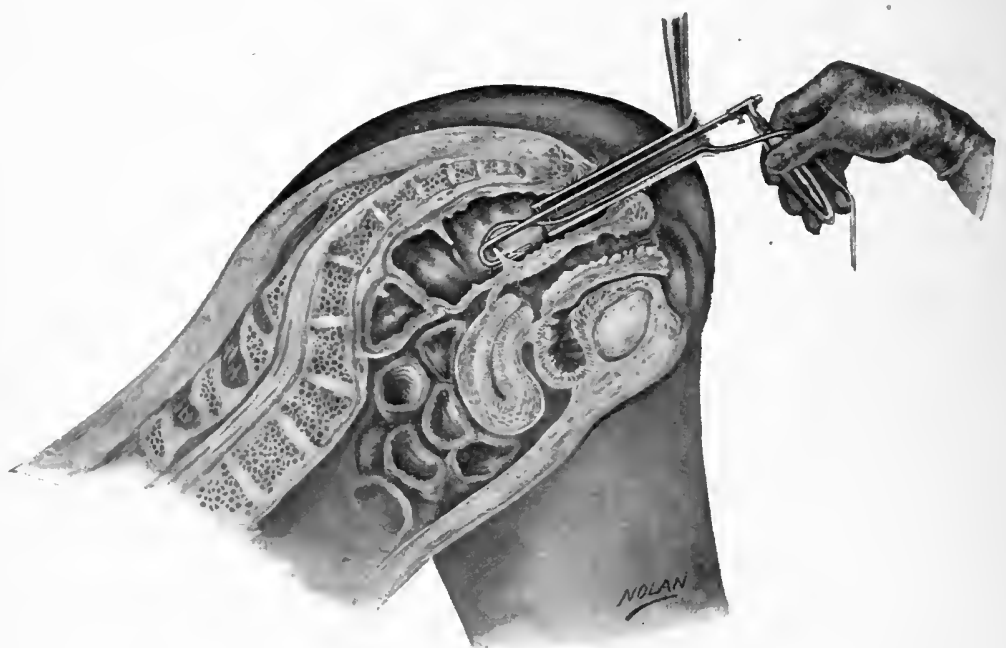

Fig. 49.

Technique of Author's Operation for Rectal Valvotomy.

This drawing shows the position of the patient in the knee-shoulder posture with the author's valvotomy needle threaded with a rubber ligature transfixing the first rectal valve. 
Fig. 50.

Author's Rubber Ligature Operation for Rectal Valvotomy.
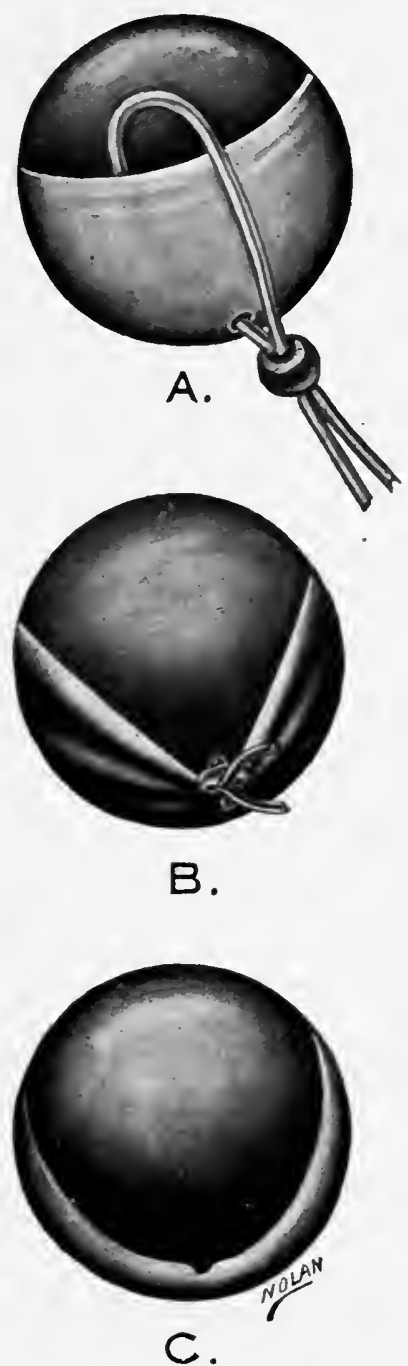

Drawn from proctoscopic view.

A. A rubber ligature in place with lead fastener ready for compression. $B$. Ligature drawn taut, and lead fastener compressed, showing amount of constriction.

C. Result three weeks after operation. 
is then grasped by means of a long alligator forcep and the ligature is pulled through until it is outside the proctoscope. The needle is then passed back and around the edge of the valve and is brought down also outside the proctoscope and is then taken off the ligature. The ligature is now in place (see figure 50, A). Over the ends is slipped a lead fastener or large perforated shot, the ligature being put on the extreme stretch and the shot grasped and pushed up to the valve tightly by means of long compression forceps and firmly compressed. This puckers the valve (see figme $50, \mathrm{~B}$ ), and constricts it in such a way that circulation is shut off and the ligature sloughs through in from two to eight days. After the ligature has cut through, the edges retract so that a large U-shaped opening is left, which gradually still further retracts. Fig. 50, C shows the retraction in cases in which the rectal valve contains a considerable amount of fibro-elastic tissue.

The advantages of this simple technique are as follows:

First. It can be done without any anesthetic whatever.

Second. It can be done quickly; that is, the whole operation should not require more than ten minutes for three valves.

Third. It requires few instruments or appliances.

Fourth. The patient is not confined to bed.

Fifth. There is absolntely no hemorrhage; no stitches are required.

Sixth. The rubber ligature, being soft and non-irritating, does not scratch or bruise the bowel in situ or during its expulsion, and there is no danger of its doing damage if it should by any possibility be carried up ligher into the bowel.

Seventh. It is simple. 


\section{CHAPTER V.}

\section{FECAL IMPACTION.}

This consists in the formation and retention in some part of the intestinal canal of a mass of hardened feces. In 70 per cent of the cases the fecal impaction is found in the rectum and in 20 per cent in the sigmoid flexure. The other 10 per cent are found in the upper portions of the intestinal canal which do not come within the scope of this work and will not be discussed.

Causes. Over-distension of the bowel caused by constipation may lead to the formation of a pouch or diverticulum. This pouch becomes filled with fecal matter and on account of the atonic condition of its muscular fibres, is unable to completely empty itself during defecation. This leads to absorption of the fluid constituents of the stool and leaves behind a hardened fecal mass whose consistency ranges from that of stiff clay to calcareons, as in enteroliths, or fecal concretions, which are composed largely of lime salts. Bits of bone, fruit and regetable seeds, fruit stones, indigestible vegetable fibre, concretions of bismuth, salol, magnesia, or other insoluble drugs, taken internally, may, become the nidus of a fecal concretion which in turn is frequently the underlying cause of fecal impaction. Gall stones may also be responsible for their formation. 
Symptoms. The symptoms of fecal impaction are those of obstipation, coming on rather suddenly with more or less intestinal distension, accompanied with pain in the rectum, and extending to the left inguinal region, and frequently shooting down the left leg. The patient will complain of a frequent desire for stool, but inabilty to accomplish the same on account of a sense of weight and blocking up of the rectum. If the impaction is low he may feel it impinging on the anus following the effort at expulsion. The pressure on, and irritation of the mucous membrane, cansed by the presence of this hard foreign body, starts up a hypersecretion of mucus and causes ulceration of the bowel. This causes in many instances a diarrhoea characterized by frequent, small, irritating, watery, and mucous stools which often contain blood and frequently pus.

Cases have been reported in which the impaction has become channelled, where after a period of almost complete obstruction, the patients have had stools apparently normal. In cases where the impaction occurs in a pouch, or diverticulum, this may also occur. In these cases, however, the feeling of weight, heaviness, and discomfort in the sigmoid or rectum is still present and there is more or less tenesmus and an unsatisfied feeling after stool.

In women, pressure from a large impaction on the uterus, or ovaries, may cause anterior displacement and symptom's of uterine irritation. 'Through direct pressure and reflexly, the bladder becomes irritable and frequent. micturition results. Patients suffering from impaction usually present in addition to the foregoing, symptoms of auto-intoxication, such as dizziness, headache, coated tongue, foul breath, indigestion, with or without vomiting, 
abdominal distension, lack of ambition, and general malaise.

Diagnosis. The diagnosis is not difficult. By rectoabdominal palpation, the round, or often nodular mass, can be made out in the lower left inguinal region, or in the rectum itself. To the examining finger in the rectun. it may be hard and nodular, or owing to its being in a pouch or diverticulum and almost completely surrounded by mucous nembrane, it may give an impression of being an extra-rectal pelvic tumor.

On direct examination with the proctoscope with the patient in the knee-shoulder position, and the rectum inflated, the impaction can be easily made ont. It is important in using the proctoscope to so carefully manipulate the instrument so as to see behind each rectal valve, as not infrequently the pouching occurs in any of these locations and the contained impaction, or concretion, is almost completely hidden from sight. If palpation discloses a mass in the sigmoid flexure examination with the sigmoidoscope may be resorted to in order to demonstrate the impaction or concretion to the eye. It is important to determine either by ocular inspection, or examination with a sound, whether we are dealing with an impaction of clay-like consistency, or a hard coneretion, as the treatment of the two is necessarily somewhat different.

Treatment. The treatment of this condition consists of the prompt removal of the impacted mass. Situated in the rectum and reached by the finger it may be easily broken up without the use of any instrument, providing it is of recent origin and its consistency not firmer than stiff clay. When it is situated beyond the reach of the 
finger or if of too firm a consistency to be easily manipulated, the injection of 8 or 10 fluid ounces of liquid albolene, olive, or cotton seed oil through a rectal tube large enough to reach well into the sigmoid, and this allowed to remain for 12 hours; will often so soften and separate the mass that it can be passed without any difficulty. In many cases this will bring the impaction down so low into the rectum that it can be broken up with the finger or a dull spoon curette used through the proctoscope with the patient in either the lateral or lithotomy position.

The most reliable method is, however, the injection of peroxide of hydrogen in solutions varying in strength from 10 to 25 per cent. With the patient in the lateral position, 2 to $4 \mathrm{oz}$ of peroxide solution is injected through a soft rubber rectal tube inserted up to the impaction. The tube is allowed to remain in place and at the end of 5 minutes the rectum irrigated; when it will be found that the impacted mass has been disintegrated through the mechanical action of the liberated gas and is easily washed out. Several injections of the peroxide solution may be necessary but if persisted in, it may be relied upon to do the work. When the mass is of long standing and so hard that it takes on the characteristics of a true concretion, it may become necessary to dilate the sphincters under local anesthesia and to break up the mass with a short jawed lithotribe passed through an operating size proctoscope. When the concretion is larger than $1 \frac{1}{2}$ inches in its widest circumference it is safest and best to administer nitrous oxide, divulse the sphincters, crush the concretion, and remove the fecal mass with forceps. 
After the impaction has been removed, the patient should be put on a liquid, absorbable diet for two or three days. Liquid albolene should be administered in doses of one or two teaspoonsful four times daily and regular daily defecations encouraged. The atonic condition of the rectum shonld be overcome by the use of the author's pneumatic massage bag as ontlined in the chapter on the treatment of chronic constipation. 


\section{CHAPTER Vl.}

\section{PRURITUS ANI.}

Pruritus Ani is probably the most annoying symptom which accompanies any disease of the recturn. It is berause of the intense suffering and discomfort which it canses when present, that it has been given the prominence and importance that is accorded it of treating it as if it were a disease by itself.

Pruritus Ani which is an accompanying symptom of so many different diseases, in reality should not be considered alone as a disease any more than rectal pain or rectal hemorrhage. Like constipation, lowever, it is such an important symptom, and often the only apparent symptom, of some diseased condition, that it has been thought wise to emphasize it in this clapter, and to speak of some of the conditions which most frequently cause it.

Pruritus Ani may be caused by or accompany every known anal or rectal disease, as well as many diseases affecting other organs or general in character. In other words, it may be caused by :

1. Any disease of the rectum or anus.

2. Any skin disease affecting the anal region.

3. As a reflex from diseases of the bladder; prostate gland, uterus, ovaries, vagina,-in fact any part of man or woman's uro-genital apparatus. 
4. General or constitutional diseases.

5. Dietary disturbances.

6. Parasites.

7. Irritation from clothing, detergents, or moisture.

The discussion of the rarious anal and rectal diseases which present Pruritus Ani as a symptom will be taken up in the respective chapters devoted to those diseases. 'The skin diseases most commonly affecting the anal region are marginal eczema, herpes, erythema, scabies, and folliculitis.

Stone in the bladder is not infrequently accompanied by an itching of the anus and perinemm. Chronic prostatitis, resiculitis, urethritis, phimosis, and cystitis may also be accompanied by itching of this region. Any disease of the uterus or adnexa may canse itching in the region of the anus and many times the symptom of pruritus is caused by some irritating discharge from the vagina.

Pediculi, thread rorms (oxyuris rermicularis), itchmite (acarus scabiei), ring worm (trichophyton), are the most common parasites manifesting their presence in the anal region by itching.

Among the diseases of a more general character which are frequently found to be the cause of itching at the anus are diabetes, malaria, uric acidosis, nephritis, tuberculosis, syphilis, and hysteria. Many patients suffer from an attack of Pruritus Ani after partaking of alcoholic stimulants in excess. In others, the excessive use of tobacco, coffee, tea and spices also conduce to the production of this symptom. Some patients are subject to attacks of Pruritus Ani only during the strawberry season, while others have an attack every time they partake of sea 
foods, particularly of the shell fish family. Some patients possess an idiosyncrasy toward some one food or class of foods, and it is the indulgence in this class only which brings on an attack of Pruritus Ani in this particular individual.

In many cases the itching is caused by mechanical ir ritation of the skin surrounding the anus or by the use of coarse or harsh material in cleansing the anus after defecation. Some writers claim, that the printer's ink on newspapers acts as a special irritant to the anus. The wearing of underwear colored with dyes which are not fast, as well as the pressure of clothing which fits too snugly in the perineal region; the irritation caused by excessive sweating, particularly in stout individuals; and those who are forced to work in a high temperature, such as engineers, stokers, monlders, and gas workers; are often responsible for the production of Pruritus Ani. Personal uncleanliness in this region is too often found to be the cause of pruritus, as in other parts of the body.

There has been a condition described by some writers as idiopathic Pruritus Ani, becanse of the presence of itching of the amus alone as the symptom, and the discovery of no other apparent cause for its existence. I do not believe that there is such a thing as idiopathic Pruritus Ani. I have seen cases in my practice where after the most painstaking and thorough search no cause could be found for the itching, yet I believe there was a local cause, only it was not discovered. The fact that some of these cases are cured empirically by stretching of the sphincter muscles rould seem to indicate that there might be some local condition irritating the nerve endings which was mechanically relieved by the stretehing process. 
The appearance of the anus and perineum in the patient suffering from Pruritus Ani is quite characteristicthe skin around the anus being thrown into numerous, deep folds radiating from the anal orifice (Fig. 51). In those cases accompanied by more or less moisture the skin is white, soggy, and more or less macerated, with here and

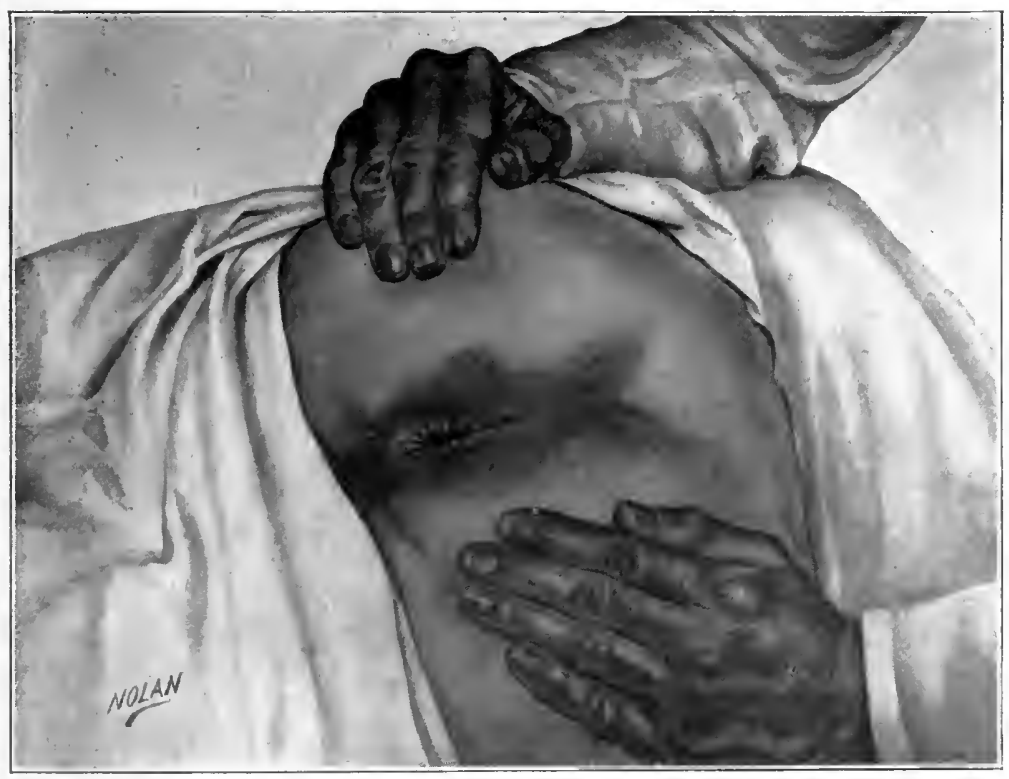

Fig. 51.

Pruritus Ani.

This drawing made from a photograph of one of the author's cases, shows the characteristic cracking around the margin of the anus and at the posterior commissure, and also shows the area of irritation of the opposing surfaces of the buttocks.

there, small raw areas where the skin has been denuded of epithelium by scratching. In other cases of not so long standing, we find the skin around the anus normal in color but dry with a tendency to scale. The cutaneous folds are not so deep, but in the sulci are found small cracks 
in the skin and extending up into the mucous membrane. In many cases particularly in stout individuals, a long raw fissure or crack may be found extending along the median raphe anteriorally to the scrotum or posteriorly into the median perineal crease for a distance of from one

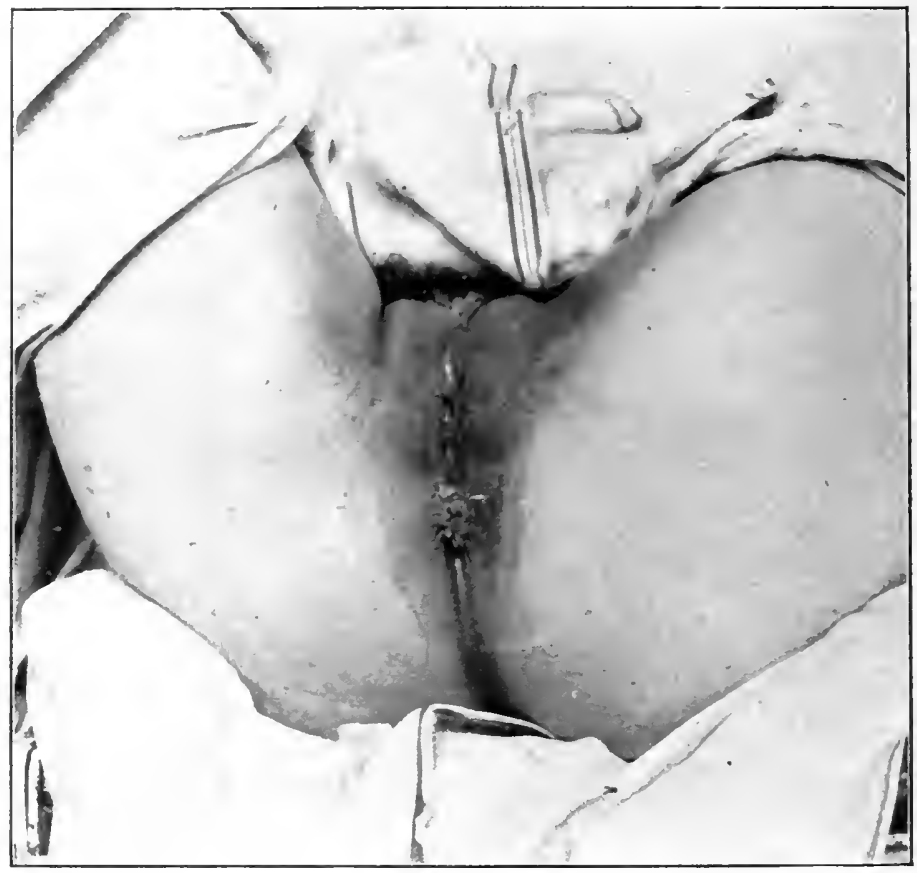

Fig. 52.

External Integumentary Hemorrhoids Accompanied by Pruritus Ani.

This photograph of one of the author's cases, shows the extent to which cutaneous irritation may go; in this case extending up over the sacrum and down nearly half way to the knees.

to four or fire inches. The skin surrounding the anus and these various cracks may be reddened and excoriated for a great distance from the lesion. It may extend some distance up onto the abdomen or down the thighs (Fig. 
52) and legs to the knees In cases of long standing the skin surrounding the anus loses its elasticity and becomes lard, thick, and leathery. This condition is in reality due more to the scratching and rubbing by the patient in his futile efforts to relieve the condition, than to any pathological condition brought about by the itching itself.

Pruritus Ani may mean anything from a slight feeling of uneasiness or irritation in the anal region to an intense burning, almost crazing, itching characteristic of the most aggravated types. There are several things characteristic about this itching:

1. It is usually more intense at night.

2. It tends to become progressively worse.

3. It is not relieved by scratching.

4. In spite of the fact that the sufferer soon realizes that the scratching or rubbing only aggravates the condition, he persistently and constantly continnes to seratch.

While every disease affecting the rectum or anus may be responsible for the production of Pruritus Ani, those that most commonly cause it are fissure of the anus, nlcer, particularly of the anal canal; fistula-in-ano either complete or blind, hypertrophied papillae and proctitis. The reader is referred to the respective chapters describing these conditions with their diagnosis and treatment. Every case of Pruritus Ani demands the most careful investigation. into the patient's habits, occupation, and mode of living; as well as the most thorongh examination of the anus, rectum, sigmoid, and adjoining organs.

Unfortunately in some few cases where pathological conditions have been found in the anus or rectum, which were thonght to be the cause of Pruritus Ani, their removal has not relieved the itching. In fact, on account of 
the healing by gramulation and the resultant scar tissue, some cases have been reported in which the itching has been aggravated. It is important, therefore, to be very guarded in the prognosis and not promise a cure.

The treatment of Pruritus Ani is of course the treatment of the disease, whether local or general, which causes it; and the reader must use his general medical knowledge in the treatment of diseases of a constitutional nature and in treating those of the general diseases mentioned above, as that does not come within the scope of this work. The treatment of the symptom itching, must be simply palliative, while the treatment of the condition which is responsible for the itching is being carried out. If due to any of the rectal or anal diseases mentioned herein, follow out the treatment as laid down in the various chapters. If due to any skin disease of the part such as marginal eczema, consult any good work on dermatology and treat it as you would any other skin disease in any part of the body. The author has found the following ointment a most successful one in these cases:

IY

Pv. Calamine _............. 2 drachms.

Zinc Oxide _............... 1 drachm.

Calomel _..._._.

Ac. Pheni: _..._.

Lanolin

1 ounce.

M. Ft. Unguentum.

This is applied freely to the parts after cleansing and thoroughly drying, after each bowel movement and at night. In some cases where there is considerable moisture the following powder may be used instead of the ointment: 
F

Chloretone 30 grains.

Pv. Calamine 2 drachms.

Zine Oxide 1 drachm.

Calomel M. \& Ft. dusting powder. 30 grains.

This is applied in the same manner as the ointment. Herpes and erythema of the skin surrounding the anus nuy be relieved by the application of the Compound Stearate of Zine with Balsam Peru. The parts must be protected and the surfaces kept from rubbing against each other by absorbent cotton. Scabies is best treated by the ordinary sulphur ointment of the pharmacopoeia. Where inflammation of the hair follicles exists with the formation of pustules they must be opened, washed with a 25 per cent solution of peroxide of hydrogen and then dressed with a compress of any of the standard antiseptic solutions, boracic acid being used by the author. Where the pediculi pubis is suspected, liberal applications of blue ointment or fluid extract of larkspur should be used. In ring worm, the trichophyton may be reached by sulphur ointment. Where thread worms are present, lime water enemata will very quickly relieve. It should be injected twice daily using from 4 ounces to $1 / 2$-pint at each sitting and capsules containing one-lialf grain calcium sulphide, given three times daily before meals.

In cases where excessive indulgence in smoking, alcoholic stimulants, and articles of diet which produce $\mathrm{Or}^{\circ}$ aggravate itching is responsible; it is obvious that these indulgences must be interdicted. Where the occupation or habits: are at fault, changes are necessary in order to bring about the best results. The remedies or combina- 
tion of remedies which are recommended for Pruritus Ani are many. Blackwash is recommended by many authorities as an old reliable remedy. Tuttle considers carbolic acid in ointment or solution from five to twenty per cent as the most generally applicable of all drugs for the relief of Pruritus Ani. He recommends this prescription :

$\mathrm{R}$

Ac. earbolici _._._._._._._._- 2 drachms.

Ac. salicylici _._._._-_._-_._- 1 drachm.

Glycerine _..._............ 1 drachm.

M. sec. art.

Sig.-Apply to the parts with camels' hair brush or cotton swab softened in hot water.

Cripps recommends :

IF

Acidi Carbolici _._._._._____e drachm.

Unguentum hydrarg. nitratis_-_ 2 drachms.

Ung. Petrolii _._._._._._._. 1 ounce.

Another ointment of which he speaks very highly of is: Ii

Extracti conii -_._-_-_-_-_-_ 1 drachm.

Olei ricini _._._._._._._._._._. 1 drachm.

Ung. lamolini, ad_-_-_-_-_-_-_- 1 ounce.

Where ointments do not agree Kelsey recommends this lotion:

$\mathrm{B}$

Sodii biboratis _._._.___.___ 2 drachms.

Morphiae hydrochlor _-__-____16 grains.

Acidi hydrocyanic Dil_____-_1/2 ounce.

Glycerine -_.

Aquae, ad______________ 8 ounces.

Cripps also recommends a lotion containing 2 grains of bichloride of mercm to the ounce of lime water as an 
application, after thoroughly washing the parts with soap and water.

Gant recommends as a hard ointment the following:

R

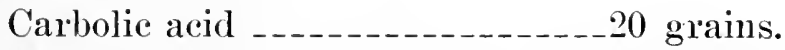

Nenthol _-_-_-_._.

Camphor - -

Suet _._._._-_._- 1 ounce.

M. Sig: Apply freely 2 or 3 times daily after cleansing the parts. In the preparation of the above he advises to melt the suet and when partly cooled to add the other ingredients. He especially cautions against adding oil as the ointment should be quite hard, the object being to form a coating over the parts which will not be penetrated by the secretions. Citrine ointment (unguentum hydrargyri nitratis) is highly recommended by Gant in cases where it is necessary to restore the circulation and the indurated skin to its normal color and suppleness. Through the suggestion of Dr. L. H. Adler, Jr., Gant uses it in the following manner: After the parts have been bathed in warm water the citrine ointment (which may have to be weakened in some cases by the addition of lard) should be spread on several thicknesses of gauze, applied, covered with oiled silk, and held in place by a snug T-bandage. This ointment should be kept on constantly, or in some cases it may be found necessary to alternate it with an ointment containing 20 grains of calomel to an ounce of petrolatum.

In the author's experience for the mere relief of itching, compresses or enemas of water as hot as can be borne has given the greatest relief in the greatest number of cases. Sometimes cold acts better than hot. An ointment 
containing twenty-five per cent of chloretone in white cold cream has proven very efficacious in the author's hands for the same purpose.

In cases presenting a fissured condition of the anus skin and mucous membrane, the application of 100 per cent solution of nitrate of silver will cause a desquamation of the entire surface within 24 hours. Then a 5 per cent solution of ichthyol in flexible collodion is applied on alternate days. The use of a mechanical vibrator, using a cone-shaped vibratode for five minutes at a time, using from 5000 to 7000 strokes a minute and inserted as far as can be borne by the patient, will often afford much relief. Firm pressure by means of a lard rubber rectal plug affords relief to some individuals where all other measures have failed. It must be borne in mind that while any of the remedies mentioned herein are being used to relieve the itching, that they are but palliative, and the permanent relief of the itching comes only after the diagnosis and cure of the condition which causes it. This must be diagnosed and studied for treatment and if the condition is not amenable to nonsurgical treatment or operative treatment under local anesthesia it is more likeIy a case for the proctologist than for the general practioner, and his aid should be called in.

If the itching is caused by the discharge from rectal cancer or from the small, shallow nlcerations of the mucous membrane between the sphincters, which Wallis of London claims is the cause of 90 per cent of all cases of true Pruritus Ani-then the indicated surgical procedures should be carried out, whereupon the itching will be relieved. The writer would suggest that one should carefully read over the clapters on constipation, anal 
fissure and ulcer, fistula, hemorrhoids, and hypertrophied papillae, as well as the chapter on the examination of the patient before attempting to treat a case presenting Pruritus Ani as a symptom.

In many cases, the local condition seems to imperatively demand surgical treatment, and in many of these patients prompt relief is experienced after the indicated operation. The author describes below those which he can safely recommend.

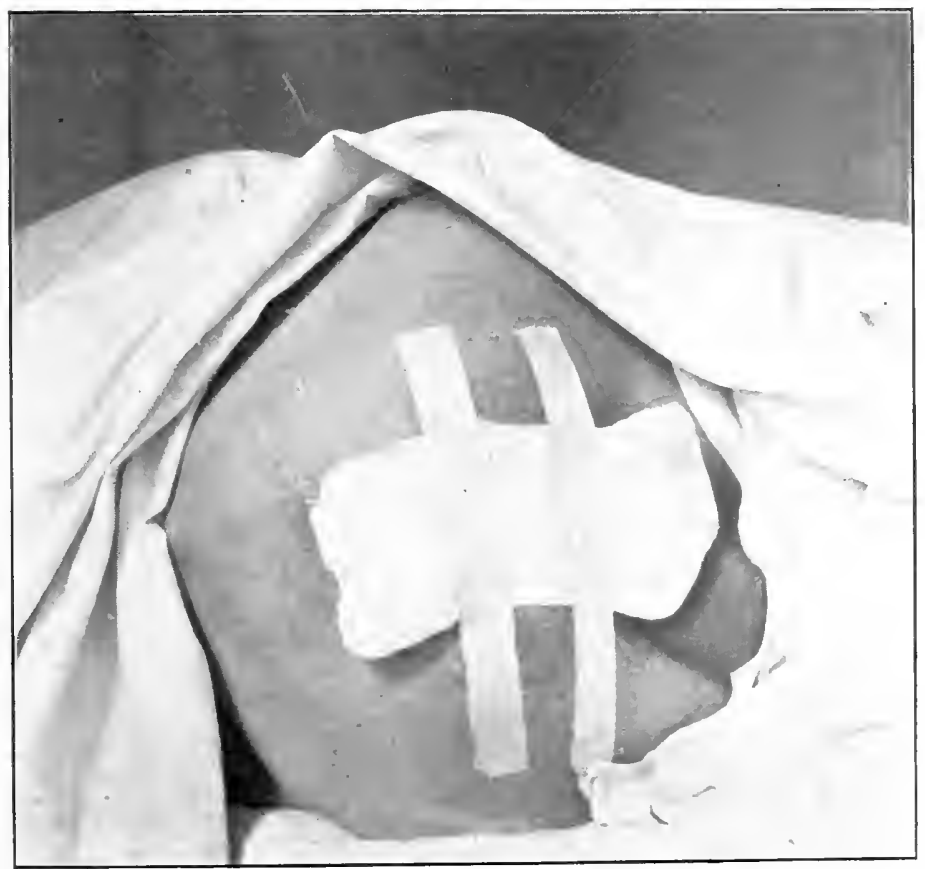

Fig. 53.

A Simple and Satisfactory Rectal Dressing.

Consisting of a gauze covered cotton pad and two strips of adhesive plaster. 
Surgical Measures. In those cases of Pruritus Ani in which the skin surrounding the anal orifice has been hypertrophied and thrown into heavy folds and the sulci between these folds fissured, irritated, and giving forth an irritating discharge; a simple surgical procedure will often give relief. E. A. Hamilton, of Columbus, O., advises the removal of these hypertrophied skin folds under local anesthesia, and reports very good results from his method.

Where there are only two or three folds involved, they can all be removed at one sitting. Otherwise, the operation may lave to be done at different sittings, with intervals between long enough to allow of complete healing of the ones already operated upon.

After cleansing, sterilizing and shaving the parts, the patient is placed either in the lithotomy or lateral position. Each fold to be removed is injected from its outermost point with $1 / 2$ to 1 per cent solution of eucain lactate. After allowing a couple of min-

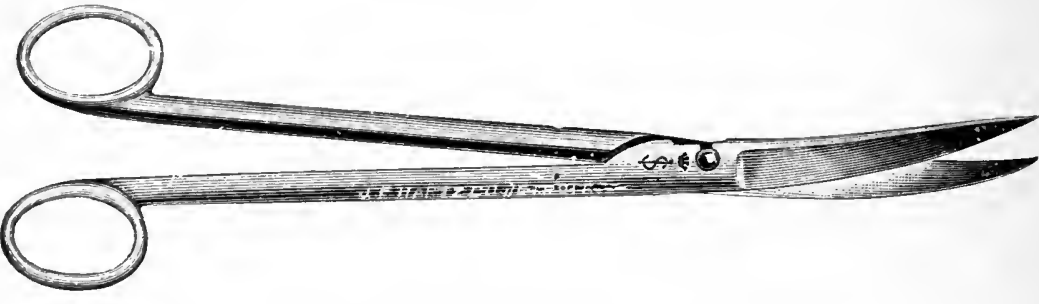

Fig. 54.

Sharp Pointed Scissors Curved on the Flat.

utes for the anesthetic to take effect, the fold is removed by grasping its apex with a pair of forceps and cutting it out at its base with a sharp scissors curved upon the flat or by elliptical incisions with the scal- 
pel. The other fold or folds are treated in like manner and the wound surfaces allowed to heal by granulation. The bowels are kept confined for three days, and then moved by the administration of a heaping teaspoonful of compound licorice powder on the evening of the third day, followed the next morning by an oil enema of six or eight ounces. Applications of bovinine three or four times daily to the wound surfaces will greatly hasten healing. After two or three weeks another two or three folds, preferably those situated opposite to those previously removed, can be dealt with in a like manner and the same technique carried ont until all the redundant tissue has been removed.

Where the pruritus is most persistent at the posterior commissure of the anus, and examination at that point shows either nothing but a thickened and irritated area extending a short way into the anal canal, or shallow excoriations at the anal margin which are neither fissures or ulcerations; the removal of a triangular flap of skin at this point is often followed by relief of the symp. toms.

The technique is as follows: After cleansing, sterilizing and shaving the parts, a point three-quarters of an inch behind the posterior commissure is selected and onehalf of one per cent solution of eucain lactate injected so as to include a triangle whose apex is the point of injection and whose base extends from one-quarter to onehalf inch to either side of the posterior anal commissure. The infiltration of the anesthetic solution shonld extend up into the anal canal far enough to include any excoriated or irritated areas. A triangular flap of skin is dissected up by means of the sharp scalpel or sharp pointed 
scissors curved upon the flat-starting at the point of injection and extending to the posterior margin of the anus. The incisions then should be brought towards each other so as to meet at a point one-quarter of an inch above the diseased area in the anal canal. The latter part of the operation makes a short, broad triangle whose base is the same as the base of a longer one on the skin surface. This leaves a dennded area kite-shaped as it were. The skin is brought together hy three or four No. 1 or 2 chromicized cat gut sutures, boro-chloretone powder applied, and the wound protected with a gauze pad held in place by adliesive strips. (Fig. 5\%.) The care of the bowels is the same as that outlined above, and the after treatment consists of daily cleansing of the parts and re-application of boro-chloretone or compound stearate of zinc powder. Healing will take place in from four to seven days and the relief experienced hy the patient after this procedure in selected cases is very satisfactory indeed.

Ball's Operation.-Perhaps the most successful surgical measure for the relief of persistent Pruritus Ani, which is available for employment under local anesthesia, is the ingenious operation devised by Sir Charles Ball, of Dublin.

As described in Ball's work on "The Rectum.", its employment is advocated under general anesthesia. The anthor, however, has been able to perform the operation with brilliant results by the employment of local anesthesia. 'Tle object of the operation is for the purpose of dividing all the sensory nerve twigs supplying the skin of the anus, anal canal and circum-anal region; which arise from branches of the third and fourth sacral nerves, come 
down to the levator ani muscle, and reach the skin by perforating the external sphincter.

The technique as employed by the author is as follows:

The patient is given a hypodermic injection of onequarter grain of morphine and 1-150 grain of atropine and is placed in the left lateral or Sims' position, and the area surrounding the anus cleansed, shaved and sterilized.

An ounce of one-eighth of one per cent solution of beta eucain lactate should be prepared and in readiness. 'Ten or twelve sharp pointed curved needles each threaded with No. 2 chromicized cat gut; a couple of sharp, small bladed scalpels; sharp pointed scissors curved on the flat; two pairs of T-forceps, and two or three hemosta-

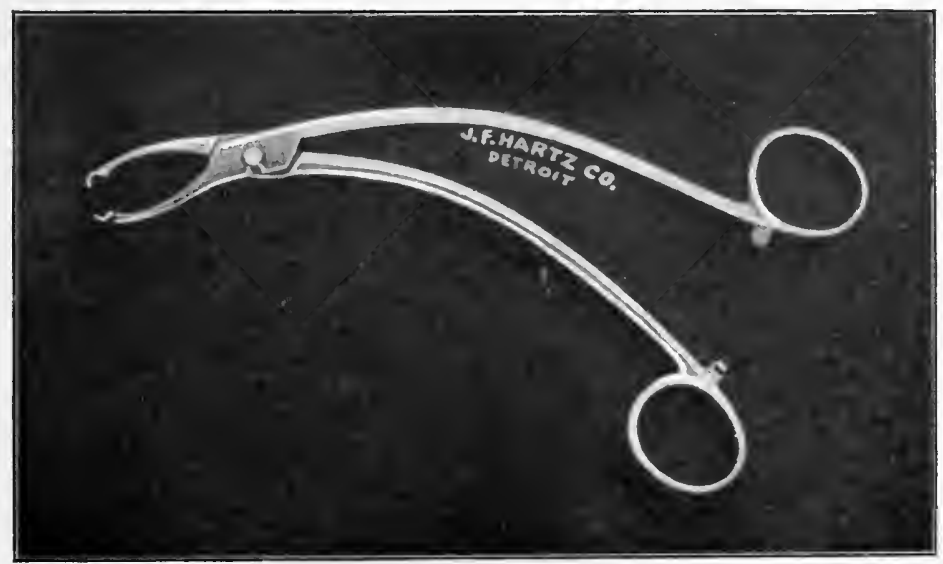

Fig. 55.

\section{T-Forceps.}

ties, and the syringe for injecting the solution are all the instruments required. Selecting the point about one-half inch behind the posterior extremity of the lines of incision in Fig. 56, the skin and subcutaneous tissue is 
infiltrated. From this point the area included inside the lines in Fig. 56 and for one-half inch beyond, is distended until complete anesthesia is secured up to the anorectal juncture. The presence or absence of skin sensibility to pain should be tested before starting to operate.

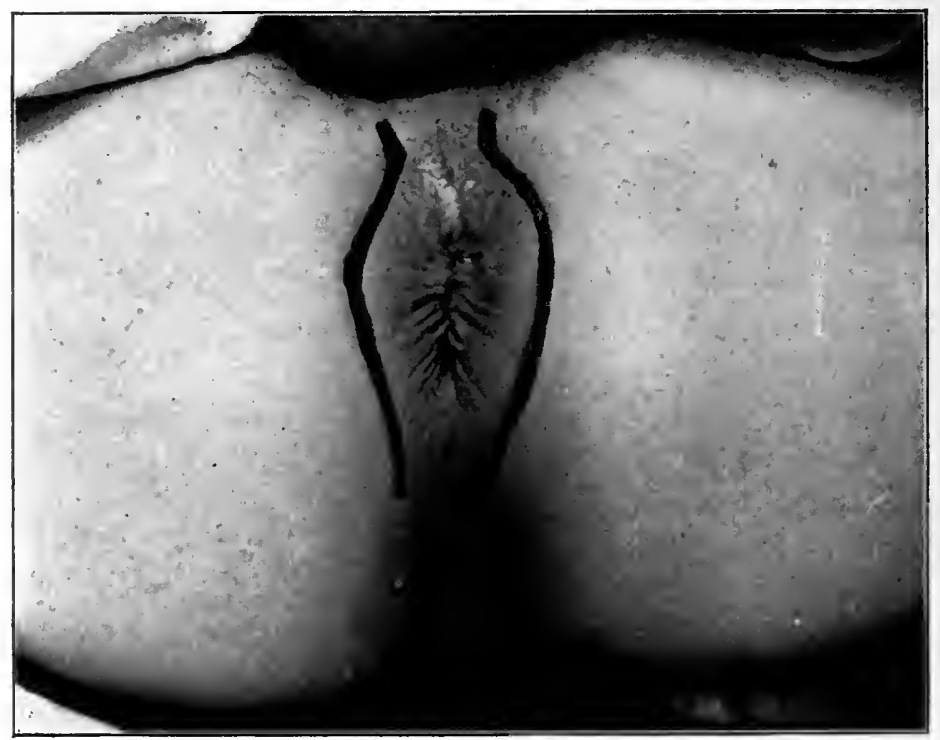

Fig. 56.

Ball's Operation for Pruritis Ani.

Elliptical lines of incision on either side of the anus. From a photograph of one of the author's cases.

The incisions, as ontlined in the above illustration, are then made with a sharp knife down through the skin to the subcutaneons tissue. The area inchded between the lines of incision should be of elliptical shape, and about twice as long in the antero-posterior direction as it is broad in the lateral, with the anal canal as its center. With the patient in the left lateral position, the incision 
on the left side is made first, the inner flap of skin is grasped with T-forceps, and by rapid and careful dissection with the scalpel is raised from the surface of the external sphincter muscle and freed up to the ano-rectal juncture. The anterior and posterior pedicles between the ends of the incisions are freed from the subcutaneous tissues as well. In other words, all connection between the funnel-shaped cutaneous and muco-cutaneous cover-

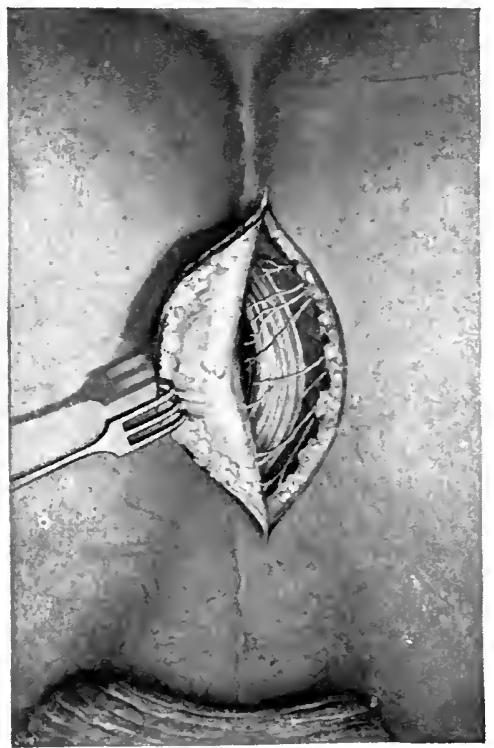

Fig. 57.

Ball's Operation for Inveterate Pruritus Ani.

Method of dissecting the flaps and of dividing the terminal cutaneous nerve twigs, which, for the purpose of clearness are somewhat exaggerated in the drawing.

-The Rectum: Its Diseases and Developmental Defects. By Sir Charles Ball.

ing of the anus and anal canal are freed entirely from their underlying tissues (Fig. 57). Ball adrocates the use of the scissors for this work, but the author has found he 
can work much more rapidly and with more assurance of dividing all the sensory nerve twig's by the use of a sharp scalpel. All bleeding should be controlled by pressure with dry gauze, and the flaps sutured again to the surrounding skin with silk worm or No. 2 chromicized catgut. Four to six interrupted sutures are all that are necessary for each incision. Firm pressure by wedge-

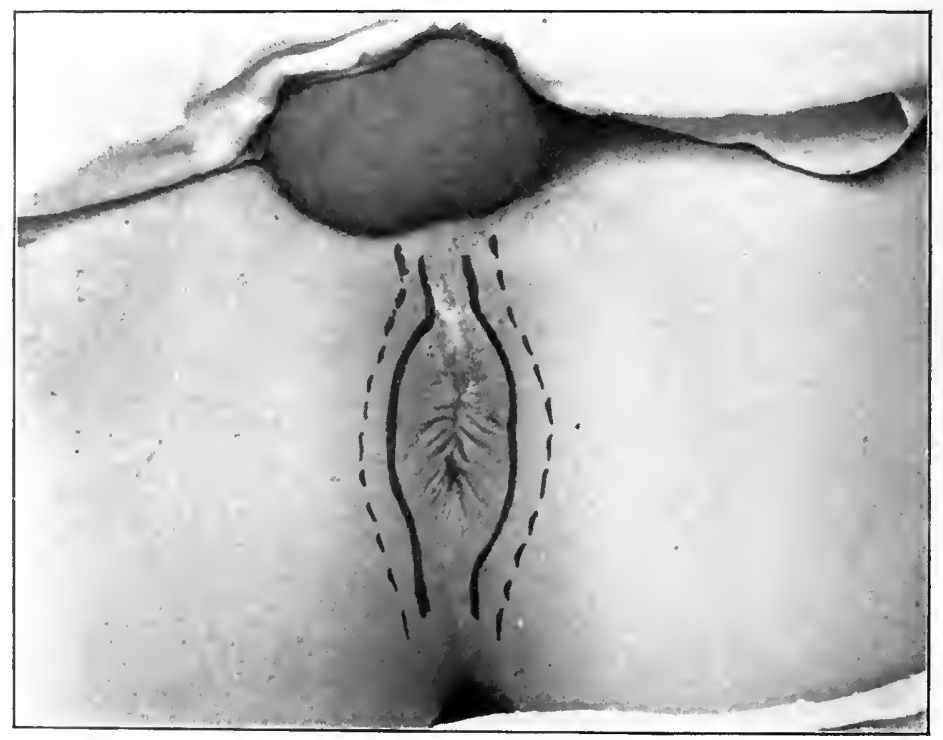

Fig. 58.

Ball's Operation for Pruritus Ani.

The dotted lines, outside of the lines of incision, show the area to which the wound is undercut, and the outside limits of anesthesia produced by the operation.

From a photograph of one of the author's cases.

shaped gauze pads is brought to bear against the region operated upon, and the dressings held in place by adhesive plaster and a 'T-bandage. This operation by dividing all of the sensory nerve branches supplying the area 
most often involved immediately renders this region superficially anesthetic, and the pruritus is relieved at once (Fig. 58). Cutaneous sensation returns after a few months, but pruritus is permanently relieved.

The after care consists in keeping the patient on an absorbable liquid diet and keeping the bowels confined for four or five days, when they are moved by an oil enema. The parts are carefully washed and kept protected at all times by the liberal use of compound stearate of zinc powder. The patient should be kept in bed for a day or two and then allowed to be up and about, but not to resume his regular occupation for a week or ten days. In the experience of the author, the results following this operation have been most happy, particularly in those old chronic cases where all other forms of treatment have been tried and found wanting. 


\section{CHAPTER VII.}

\section{ANAL FISSURE AND ULCER.}

Anal fissure, or fissure-in-ano, is probably responsible for more acute pain, suffering and discomfort than any other lesion of its size occurring in the human body. The

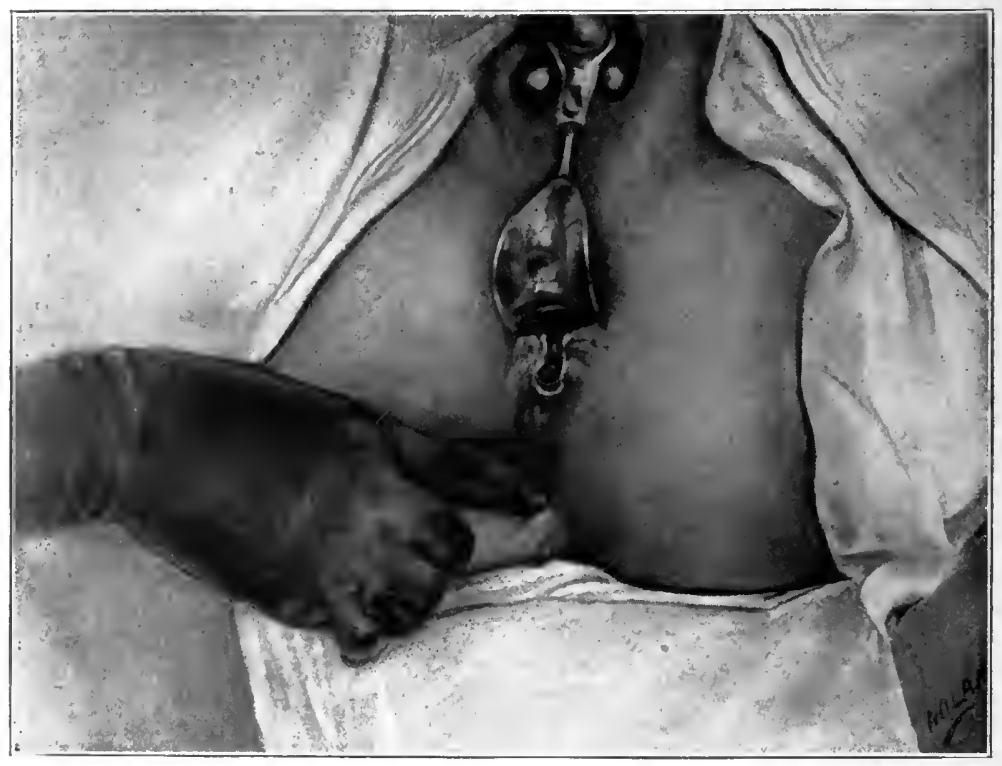

Fig. 59.

Fissure of the Anus.

This is a drawing of an old chronic case occurring in the author's practice and well shows the extent to which the ulceration goes in some cases. A well developed sentinel pile will be seen at the lower extremity of the fissure. 
fissure, as its name implies, is a crack or elongated ulceration, occurring most frequently at the posterior commissure of the anus (Fig. 59).

Cause. Fissures are caused by trauma. The traumatism may be produced either by the passage of an unusually large stool, introduction $\mathrm{or}^{*}$ expulsion of a foreign body, sneezing, coughing, or by faulty instrumentation. Fissures are usually single. When more than one is present it is an evidence, as a general rule, of the presence of tubercular, gonorrhoeal or șphilitic infection, or a

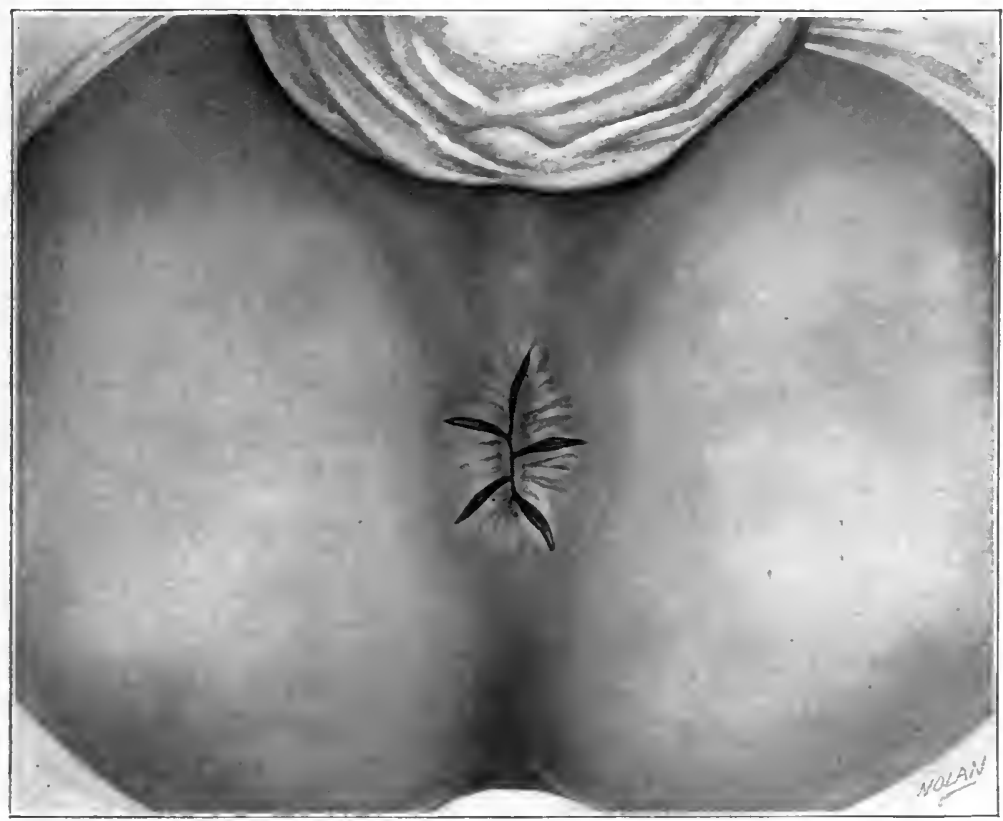

Fig. 60 .

Multiple Fissure in Ano.

This shows the extreme to which anal fissures may go in cases suffering from wasting diseases.

Drawn from one of the author's cases. 
run-down condition caused by some one of the wasting diseases (Fig. 60).

In men, in 90 per cent of the cases the fissure will be found at, or just at one side of, the posterior anal commissure. In women, about 60 per cent-the other location being at, or to one side, of the anterior commissure.

The reasons that the posterior commissure is the most frequent location for fissure are : The fact that on account of the concavity of the sacrum the curvature of the rectal and anal canal is such, that the greatest force during the expulsion of the stool is towards the posterior commissure. Also, the fact must be remembered, that the fibres of the sphincter ani muscle run parallel to each other posteriorily (see Fig. 4), to the coccyx; and this is the direction of the anal line of cleavage. Moreover, this is a constant location for one of the crypts of Morgagni, and the tearing down of a semi-lunar valve at this point (Fig. 61), is also an important etiological factor in the production of fissmre.

Any inflammatory condition which will cause a moisture and softening of the anal skin will render it more liable to be injured during a movement, and fissure prodnced. A fissure is, in reality, a longitudinal ulcer. When the fissure has been in existence for some time, it tends to become clronic and the tissues surrounding it become indurated, and the skin is pushed down in the form of a tag which becomes hypertrophied (Figs. 59 and 61) in such a way as to give rise to a thick crescentic fold known as the "sentinel pile." Fissures frequently are found accompanying hemorrhoids, the ulceration being located in the sulcus between two hemorrhoidal masses. Not in- 
frequently when the fissure is of the chronic variety, it is accompanied by a polypus, which by hanging down into the fissure from its upper extremity, tends to keep

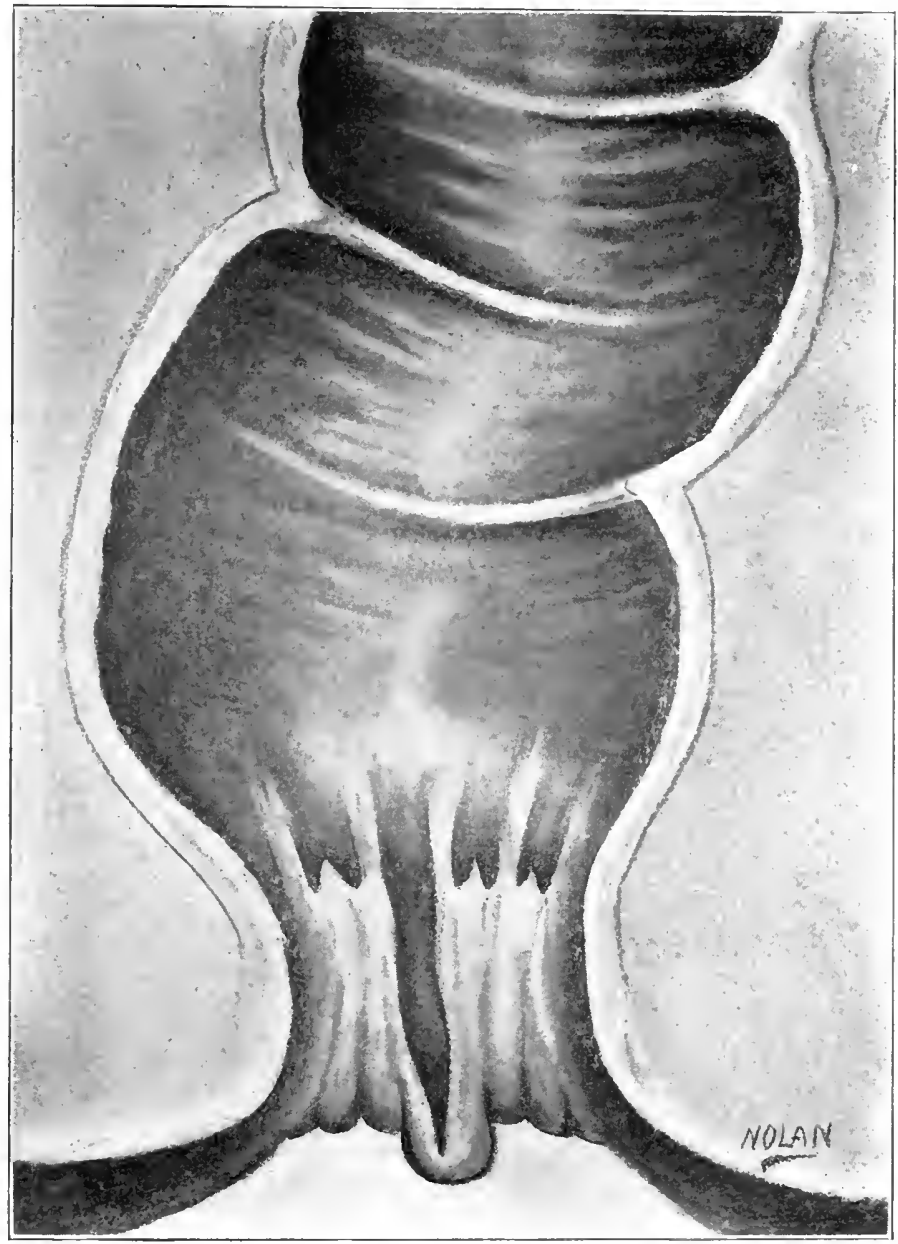

Fig. 61.

Fissure in Ano Resulting From the Tearing Down of One of the Crypts of Morgagni With the Formation of a "Sentinel Pile." 
it irritated and prevents it from healing. One reason advanced for the fact that fissures or ulcerations in the anal canal tend to become chronic rather than to heal. is the fact that the anal canal is lined by a layer of thin transitional epithelium which is neither mucons membrane nor skin, and is poorly supplied wth blood. This fact, and the action of the sphincters keeping the parts in motion, tend to prevent good healing.

The diagnosis of fissure is comparatively easy. A patient presenting himself with a history of sharp, cutting, often excruciating pain, accompanying the passage of a hard stool, and the appearance of hemorrhage following the passage, is in itself almost pathognomonic of the production of a fissure. Added to this the history of pain, usually very severe, as well as the appearance of blood with each snceeding stool, is corroborative. Then the patient also complains of a beating, throbbing pain lasting from half an hour to several hours following the passage and painful spasmodic contractions of the anal sphincter, or Pruritus Ani, the diagnosis of fissure-in-ano is almost conclusive, without an examination. However one can never take the diagnosis of any condition in the anal or rectal region for granted, withont making a thorongh examination. Therefore, after obtaining such a history, the patient should be placed on the table in the lateral position for examination.

Upon separating the buttocks, the first thing that will usually attract attention, except in acute cases, is the presence of a sentinel pile. This gives a clue at once to the location of the fissure, which will be found, as above stated, almost always at, or to one side or other, of the posterior anal commissure. Inasmuch as the entire 
sphincter is inflamed, hypertrophied and exquisitely sensitive to the touch, it may be necessary, before a satisfactory examination can be made, to anesthetize the parts.

However, if by gentle traction on the skin, just below the sentinal pile, a red raw abrasion is disclosed, extending upward into the anal canal, the diagnosis of fissure is confirmed. If this procedure causes much suffering to the patient, it had better be abandoned until the sphincter has been anesthetized according to the technique outlined in Chapter $\mathrm{XV}$.

In cases which have existed for some time, the fissure instead of presenting a red angry appearance, may be covered with a grayish or yellowish exudation. The reason that a fissure or ulceration of this region is so exquis. itely tender is because of the exposure of some of the numerous nerve endings with which this region is so generously supplied. The only other condition with which fissure is liable to be confounded, is hemorrhoids, and that only from the patient's standpoint. Not infrequently practitioners have been led into the error of taking the patient's word for the fact that he was suffering from hemorrhoids, because of the symptoms of pain at stool and hemorrhage; and the author would reiterate at the risk of becoming tiresome, that a rectal examination must be made in every case, when the exact diagnosis can be easily made.

Treatment. The treatment of fissure in ano resolves itself into palliative and operative. Many cases of fis. sure of recent origin are entirely amenable to non-surgical treatment. 'The first thing to do is to relieve constipation, which is done by putting the patient on a suitable diet, excluding all such articles as leave much residue and 
cause bulky stools. The administration of white petroleum oil, suitably flavored, in doses of from four drachms to an ounce daily, will soften the stools to such an extent as to make them easy of expulsion and yet not liquid and irritating.

Where the fissure is shallow, and is not accompanied by the formation of a sentinel pile, the application of a swab moistened in four per cent encain solution, for four or five minutes, followed by the application of pure ichthyol to the surface of the fissure, is very efficacious.

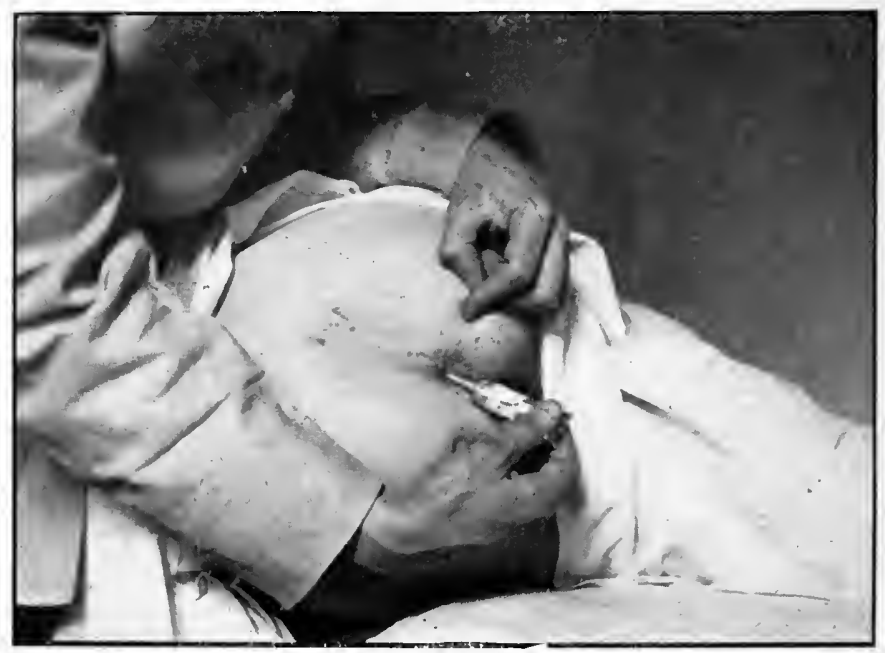

Fig. 62.

Method of Applying Ointment to the Anus from a Long Nozzled Collapsible Lead Tube.

This is repeated every second day. In the meantime the patient is instructed to carefully cleanse the parts after bowel movements and to apply, by means of a long noz. zled ointment tube (Fig. 62) the following: 
$\mathbf{R}$

Chloretone gr. xxx

Thymol iodide gre $x x$

Ichthyol gr. $\mathrm{xxx}$ Lanolin qs. $\overline{\tilde{3}} \mathrm{ss}$.

\section{Ft. Unguentum.}

In other cases, the application of a mixture containing 10 per cent of ichthyol and 8 per cent of chloretone in flexible collodion, will be sufficient. The application is made by means of a swab, or directly from the long nozzled ointment tube; the parts being separated with the thumb and forefinger of one hand, while the application is made with the other. Then the parts are thoroughly dried, and the evaporation of the ether hastened by means of the air current. Stearate of zinc powder is applied and a pledget of absorbent cotton protects the parts from the clothes.

Occasionally, where the fissure is very superficial and consists merely of a crack in the mucous membrane, a single application of a 100 per cent solution of nitrate of silver will be sufficient. This acts by causing a protective covering of albuminate of silver to be formed and effects the cure. Proper attention to the condition of the bowels, cleanliness and the application of stearate of zinc powder being all the after care that is required.

The daily application of mild solutions of nitrate of silver, alum, copper sulphate or the use of the caustic stick are not to be advised, because they only keep up the irritation and destroy the new granulation tissue as fast as it is formed. The stronger solution of silver nitrate, as mentioned above, by its sudden coagulation of the albumen of the tissues when it comes in contact with the 
wound, causes the formation of an impermeable protective covering for the granulating surface beneath; and, moreover, is far less painful than the milder solutions. Suppositories for the relief of fissure, do not appeal to the author, inasmuch as fissure is always found in the anal canal and the action of a suppository is exerted only in the lower rectal cavity; he fails to see where any direct relief can be obtained from suppositories in this condition. Moreover, it is doubtful whether an ointment applied with the finger is of any value, for it certainly cannot be applied high enough to reach any but the most dependent portion of the fissure; yet it is astonishing how often the patient suffering with fissure is dismissed with a prescription for an ointment.

Surgical Treatment. The best and surest and quickest treatment for fissure-in-ano is incision or excision. The author knows of no operative procedure in the line of proctology from which more satisfactory results are achieved than the incision or excision of an anal fissure. Under local anesthesia, this is very easily and readily accomplished, and the results are invariably all that conld be desired. In some cases, where the fissure is of recent origin, not accompanied by much inflammatory infiltration of the surrounding tissues, simple divulsion is all that is necessary to effect a cure. Divulsion of the sphincter, however, can be accomplished to the extent of temporarily paralyzing the muscle, only by the use of a general anesthetic. This can be best, quickest, and most safely accomplished by the use of nitrous oxide, than any other anesthetic.

Incision. The technique of incision of anal fissure is as follows: After anesthetizing the sphincter 
and dilating it, as outlined in the chapter on local anesthesia, a drachm or so of one-tenth of one per cent solution of eucain is injected below and around the fissure in such a way as to raise it up so that it is resting on a "water bed." After waiting a minute or two for anesthesia to become complete, an incision is made from the extreme upper end of the fissure down through the center and extending beyond the lower extremity for a quarter of an inch into the skin. The incision should be so made that its upper or inner

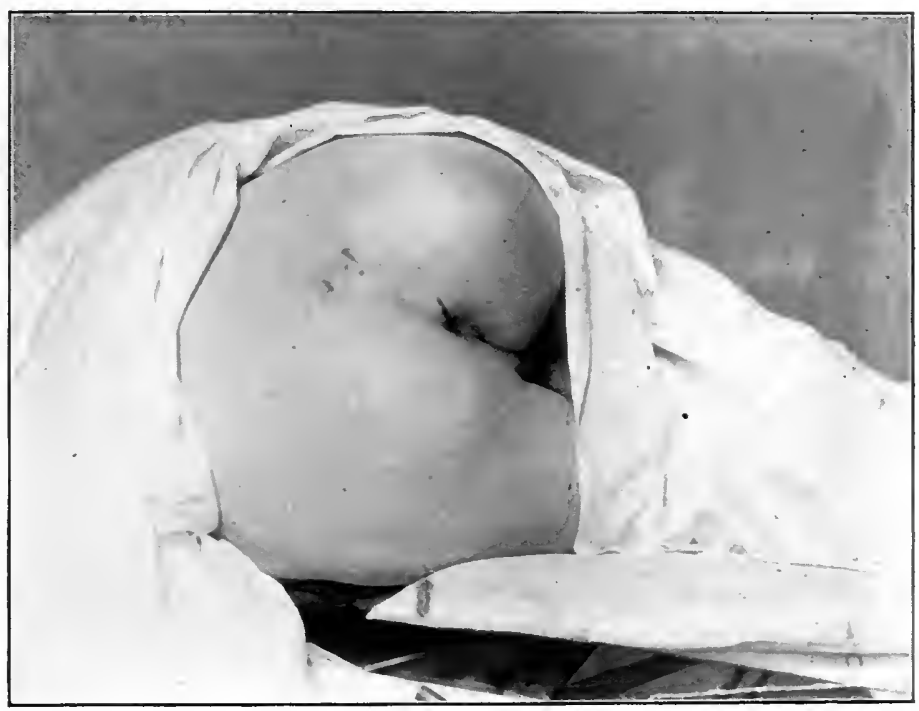

Fig. 63.

Simple Incision of Fissure in Right Posterior Lateral Quadrant of Anus.

extremity should be the shallowest, and it should become deeper until at the lower or skin end it is from onequarter to one-half inch in depth, slanting in such a way that the upper or shallowest part shall be the first 
to heal and the lower the last-thus providing proper drainage. The unhealthy surface should be lightly curretted, a suppository containing two grains each of chloretone, thymol iodide and powdered opium inserted, and a single strip of plain gauze placed in the wound.

At the end of 24 hours the ganze is removed, but the patient's bowels not allowed to move for three days at least. In the meantime, he is kept on liquid diet and the administration of white petroleum oil is started on the evening of the second day, so that the first stool will be soft and unirritating. It is advisable on the evening before a stool is desired, to administer a level teaspoonful of compound licorice powder, and the first thing the following morning, to inject through a small rubber catheter, six or eight ounces of olive oil into the rectum to insure a soft and easy movement.

The after care consists in keeping the parts clean, the bowel movements soft, and the patient up and about after the first 24 hours. If granulations become flabby or unhealthy in appearance, a single application of 100 per cent nitrate of silver is usually sufficient to stimulate healthy healing. On the other liand, if the patient is in a run-down condition and the healing slow, the insertion of a one-half inch strip of gauze soaked in bovinine, twice daily, will nourish the healing tissues and bring about a speedy result.

While in many cases this procedure will be sufficient, it will not answer where the fissure is of long standing, or if surrounded by an area of infiltration, or where there is a well developed sentinel pile or a polypus accompanying the fissure. Ofter a fissure after incision will not heal, because of the fact that the mucous membrane dips down 
into the wound and tends to keep its edges apart. To obviate this, and to make sure that all the diseased tissues are removed, the author excises instead of incises, when operating for fissure in ano.

Author's Operation. With the patient prepared and anesthetized as for the incision operation (with the exception that the area of infiltration anesthesia is made more extensive so as to include all the induration surrounding the fissure), he proceeds as follows: The fissure is grasped at its upper extremity with sharp toothed for-

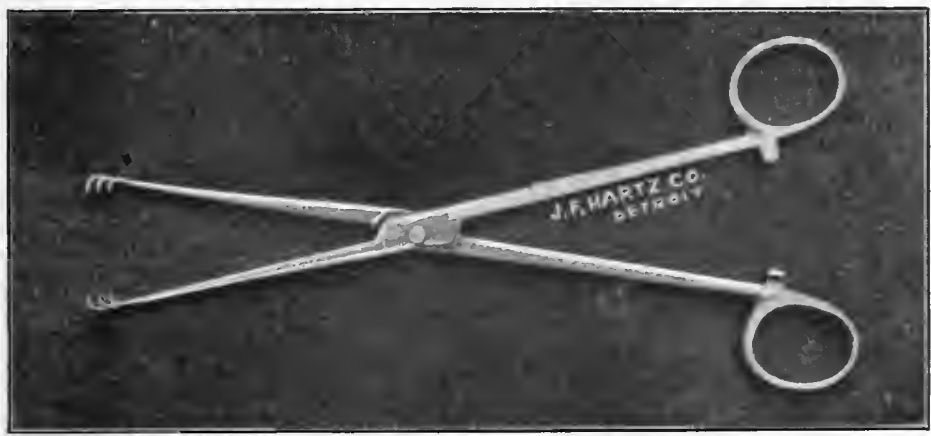

Fig. 64 .

Sharp Toothed or Pronged Forceps.

This is a very useful instrument in many ano-rectal operations and while originally designed as a tonsil forcep, is of great value in proctologic work.

ceps and two longitudinal incisions are made, one on either side of the fissure, starting from one-eighth to onefourth inch to either side of its upper or inner extremity and being made in such manner that they meet underneath the fissure in its median line, forming a $\mathrm{V}$-shaped trench (Fig. 65), as it were, which is one-eighth of an inch deep at its upper extremity and one-fourth of an inch wide; and at the outer or skin portion its width is 
from one-half to three-fourths of an inch and its depth from one-fourth to one-half inch. This disposes of the entire fissure, with its indurated edges, and the sentinel pile as well. It also allows of the fissure

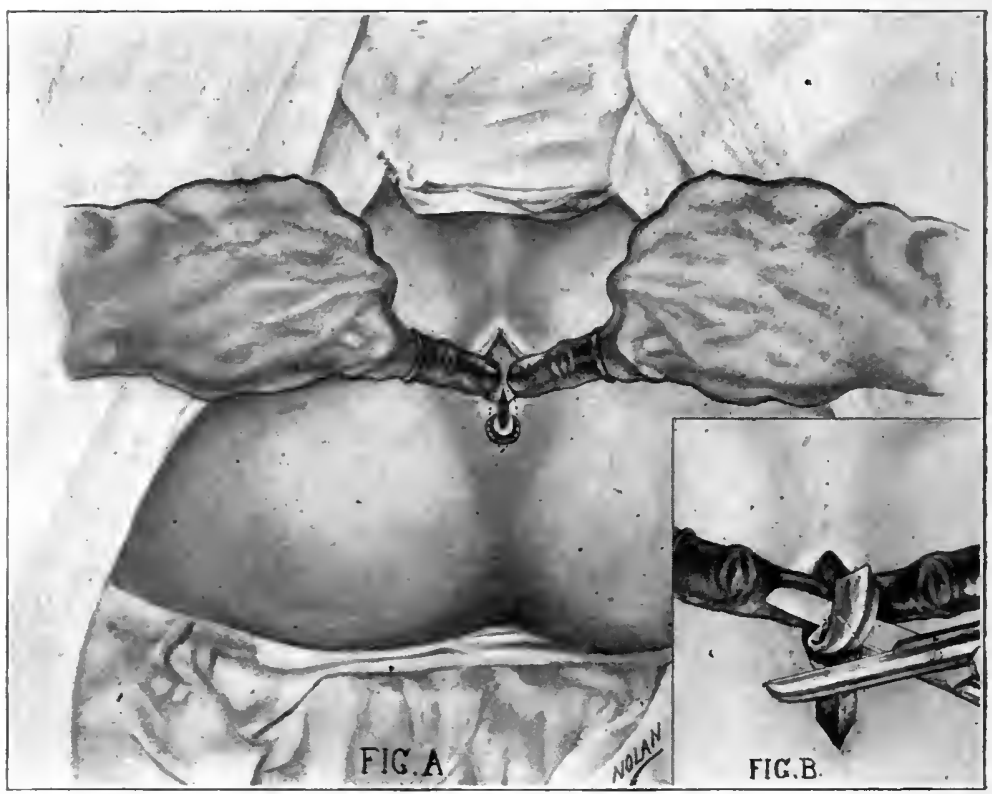

Fig. 65 .

The Author's Technique for the Excision of Anal Fissure.

$A$. The dotted lines show the line of incision both on skin surface and mucous membrane.

$B$. Showing V-shaped bed left after removal of the flap containing the fissure; the dotted lines show the shape and direction of the incision inside of the anus.

healing quickest at the bottom and prevents any overgrowth of the mucous membrane or dipping down of the edges. If a polypus is situated at the upper extremity, the incisions are carried up to include it, and as the fissure is dissected up from below, a liga- 
ture is thrown around the base of the polypus, tied, and the fisure and polypus en masse cut away. The aftertreatment is the same as outlined for the incision opera-

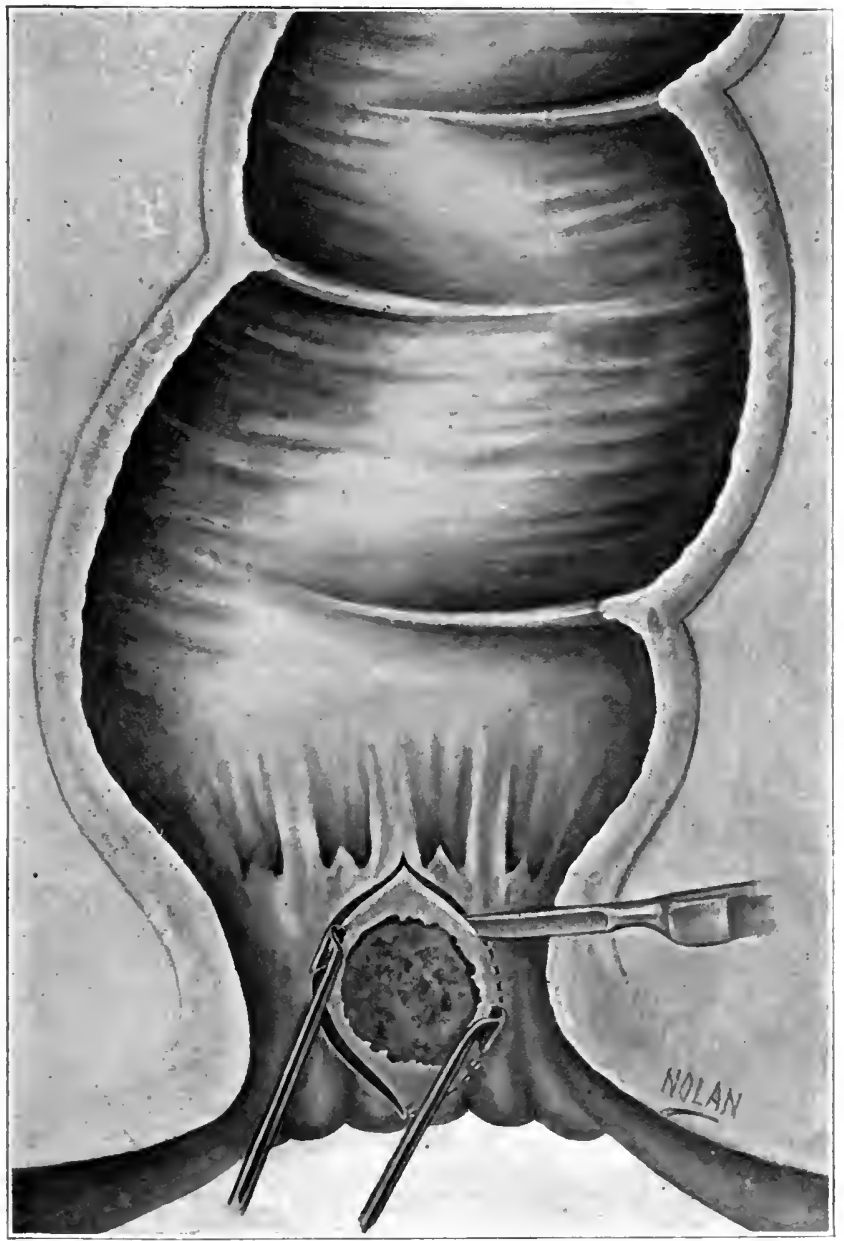

Fig. 66.

Operation for Excision of Anal Ulcer.

Note the manner in which the incisions are brought to a point at upper and lower extremities of wound. 
tion. This operation, in the hands of the author has been so satisfactory that it is his rontine treatment for all fissures not amenable to non-surgical treatment.

Anal Ulcer. Whatever has been said regarding fissure in ano in regard to treatment by non-surgical measures is equally applicable to anal ulcer, the only distinction between the two conditions being a question of the shape of the ulceration-the fissure being elongated, while the other ulcers of the rectum are round or irregular in outline. In ulcers which do not respond to the applications advocated for fissure, the injection of a few drops of one-tenth of one per cent eucain solution under the ulcer is advisable, and a light curetting of its surface will often be followed by marked relief. Where the ulcer is of long standing, the excision of the indurated tissues surrounding as well as the ulcer itself should be accomplished following the same technique as outlined for the excision of fissure, varying the direction of the incision to correspond to the shape of the ulcer (Fig. 66).

The after-treatment following excision of an anal ulcer. is exactly the same as that outlined above, following fissure. It is the watchful after-care of the conscientious plyssician following many of these minor anal operations which is responsible for the good results-for often a well executed operation is nullified in its results by neglectful, slovenly or misdirected after-care. Oftentimes the after-care of patients following these operations, is over-done rather than the reverse, and meddlesome interference accomplishes more harm than the operation does good. 


\section{CHAPTER VII.}

\section{ABSCESS OF THE ANO-REC'IAL REGION.}

The region of the anus and rectmm is peculiarly prone to infection and abscess formation, for several reasons: The unusual amount of cellular tissues surrounding the rectum; the lavish blood supply of this region; the constant presence in the rectum of progenic bacteria; the traumatism from unusually large or hard feces, foreign bodies which have been swallowed, such as spicules of bone, fruit pits, seeds, and other articles which have been ingested. The rich lymphatic supply of this region is of no small moment in the production and extension of septic inflammation. Skin diseases around the anus, particularly those which affect the hair follicles, inflammation of external hemorrhoids, the irritation from clothing or harsh detergents, disease of the cropts of Morgagni, rectal ulceration and anal fissure-all may form the starting point for the formation of an abscess in this region.

Septic infections of the ano-rectal region have been divided into different classes by difierent anthors. Tuttle classifies them as follows: 


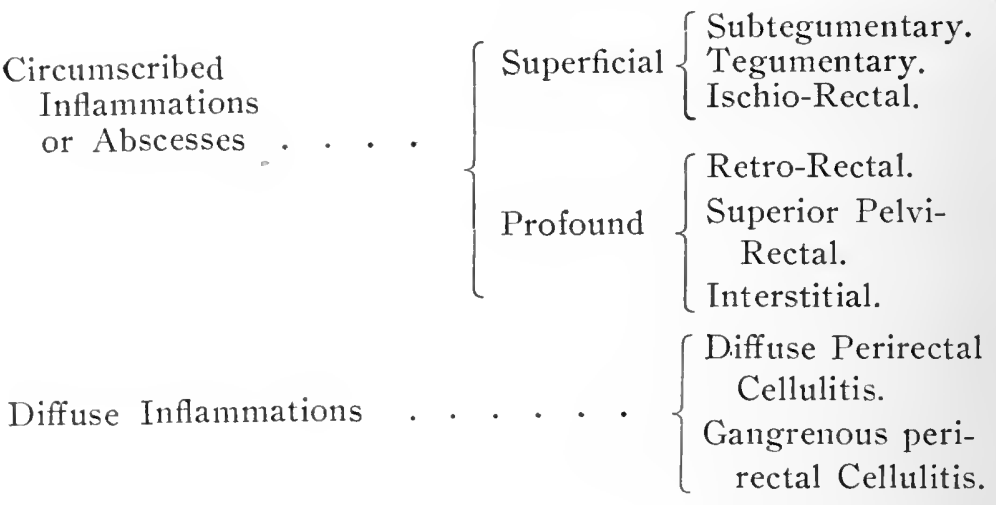

Of the circumseribed inflammations or abscesses, only those which are located below the levator ani muscle are amenable to treatment under local anesthesia and will be considered by the author under the head of tegumentary or perineal abscesses; peri-anal, marginal, or subtegumentary abscesses; submucous or intermural, and ischiorectal abscesses.

Tegumentary Abscesses. : The tegnmentary, or perineal abscesses, are really nothing more than pustules, or furuncles of the skin surrounding the anal orifice, or a pustular inflammation of the hair follicles. They may be brought about by anything which causes irritation of the parts, such as extensive perspiration; discharge from the anus or vagina; chafing from the clothing; infection by the finger-nails in scratching; personal uncleanliness, or the use of harsh detergent materials. The condition may range from a simple acne of the parts to the formation of numbers of typical boils. These cause a slight sense of irritation, smarting or itching, and canse more discomfort when the patient is sitting or walking than any interference with the function of the bowel itself. Occasion- 


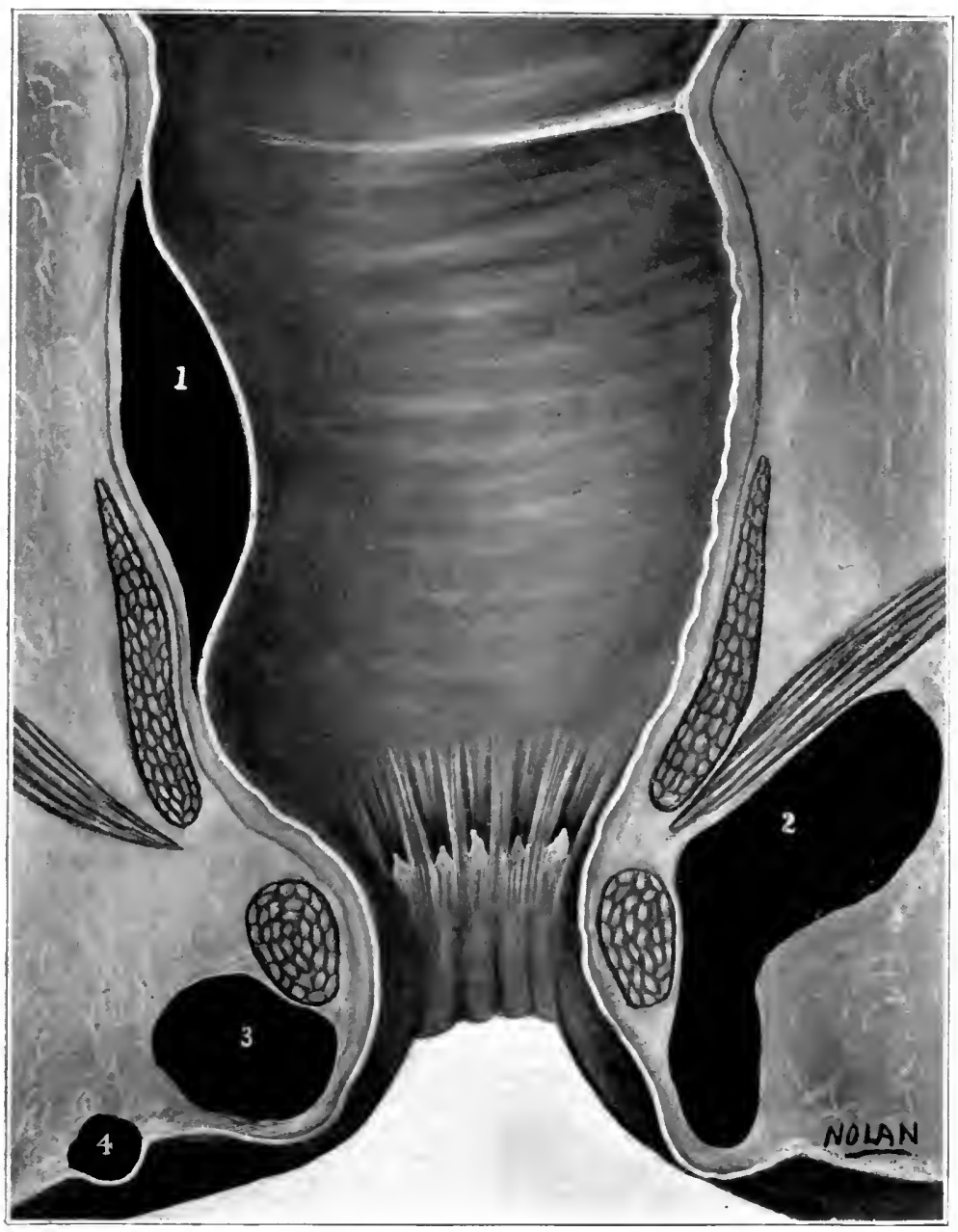

Fig. 67.

Ano-Rectal Abscesses.

1. Submucous or intramural abscess.

2. Ischio-rectal Abscess.

3. Marginal or Subcutaneous Abscess.

4. Tegumentary or Cutaneous Abscess. 
ally several of these small abscesses may run together, forming a typical carbuncle. This, however, is rather rare in this region. There is usually a slight rise of temperature, a degree or two at outside; and more or less irritability of the patient's temper. There are no constitutional symptoms.

Diagnosis.-With the patient in the lateral posture, these abscesses will be seen occurring either singly or in groups as romded reddened swellings from the size of a large pin head to a hazel nut; with or without a point of suppuration showing.

Treatment.-The treatment consists of spraying each abscess with ethyl chloride and opening with a sharp bistoury. After allowing the pus to escape, the cavity is swabed with $95 \%$ carbolic acid. Daily washing of the part with warm saturated solution of boracic acid and dressing with boro-chloretone powder will usually be all that is necessary in the line of after-treatment. The parts should be washed after defecation and protected with sterile gauze and the clothing worn loose so that there is no pressure or chafing from that source to keep up the irritation.

If there is a tendency for these little skin infections to recur, it is advisable to treat the patient with a bacterial vaccine made from the predominant germ responsible for the infection. In most cases this will be found to be the staphylococcus pyogenes aureous or albus.

Subtegumentary or Marginal Abscesses. The most common abscess developing in the region of the anus is that which occurs deeper under the layers of the skin or lining membrane of the anus, described in the above classification as subtegumentary, also known as peri-anal or 
marginal abscesses; also as subcutaneous, submucous or intra-mural, depending npon the kind of tissue under which the abscess develops. While often their starting point can be traced to a fissure or ulcer, a broken down thrombotic pile, or a diseased crypt, or the traumatism due to a bit of bone or other swallowed foreign body ; nevertheless, in many cases, the point of infection cannot be determined-leading us to the conclusion that the abscess is caused by extension through the lymphatic system, from some more or less remote injury or disease in this region. They may occur at any age, but are less common in children.

Symptoms.--Occasionally abscesses which oceur in this region, particularly the sub-mucous variety, have formed and gone on to a considerable size without causing any other symptoms than a sense of uncomfortableness or fullness in the lower rectum, noticed particularly during defecation. Usually, however, the patient complains first of sharp darting pains in the rectum, which is soon followed by an aching, throbbing pain which is persistent and gradually increasing. This aching extends to the sacral region and the pain often shoots down one or both legs, even to the heel. The patient often complains of difficulty of urination. Defecation is always painful and on account of the feeling of fullness in the rectum, is put off by the patient as long as possible. The pulse rate increases in rapidity and the temperature rises from one to four degrees. The patient cannot sit comfortably and rests his weight on one buttock or the other; a characteristic posture of patients suffering from acute rectal disease (See Fig. 8), which is almost diagnostic in itself. 
An abscess may often come on in from 24 to 36 hours, and occasionally will rupture before the patient is really aware of the severity of the trouble. These are the cases which are most frequently followed by fistula formation.

Examination.-With the patient in the lateral posture, often notling can be determined by ocular inspection unless the abscess be situated at or outside the margin of the anus-when it will appear as a rounded swelling, reddened in color, situated most often at one side or other of the posterior anal commissure. On digital examination, its outline can be definitely determined and its extent noted. If seen early, a definite point of fluctuation cannot be made out, but the whole abscess has a hard, doughy feel. It is extremely painful to the touch and, on account of the accompanying spasmodic contraction of the sphincter muscle, it is often very hard to examine.

Submucous Abscess. The submucous or inter-mural variety occur's underneath the mucous membrane covering the lower rectum, and may be found at any point in the circumference of the rectum. Those located in the anterior wall are usually accompanied by disturbances of urination. In fact, often times patients are unable to urinate at all and have to be catheterized. This variety is diagnosed by digital examination-the well lubricated finger, gently inserted through the anus while the patient is asked to bear down. A rounded mass may be felt within an incl or inch and a half of the anal outlet, either of a doughy consisteney or distinctly fluctuating. By gently sweeping the finger from side to side, the outlines can be made out and its extent determined. With the short anoscope, the diagnosis can be further confirmed (Fig. 68), 
and not infrequently the point of infection determined. Occasionally, the abscess may extend down to the integu. ment outside of the anus, forming a submuco-cutaneous abseess.

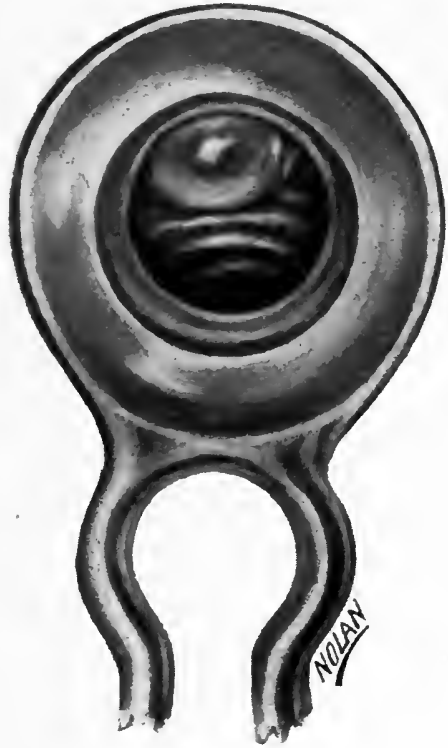

Fig. 68 .

Proctoscopic View of Submucous Abscess of the Rectum.

Diagnosis.-The diagnosis, after both digital and ocular examination, is very evident. Given the symptoms of rise in temperature, rapid pulse, aching, throbbing, pain coming on more or less suddenly in the region of the anus or lower rectum and remaining; becoming more persistent and increasing in severity, with the presence of a circumscribed painful swelling, makes the diagnosis of abscess in this region conclusive.

Treatment.--The treatment of the sub-cutaneous or marginal variety is very satisfactorily accomplished un- 
der local anesthesia. If the abscess is situated at or below the juncture of the anus and rectum, it will not be necessary to anesthetize the sphincter muscle. With the patient in the lateral or lithotomy position, the parts are scrubbed, shaved and sterilized, and the skin over the abscess injected with $1 / 2$ of one per cent solution of beta eucain lactate. A point a half-inch below the abscess proper is selected for the first injection, and the injection carried upward so that a wheal or welt a quarter of an inch to half an inch wide, and extending the entire length of the abscess, is formed. After waiting two minutes for the anesthetic to take effect, an incision is made from one extreme of the abscess to the other in a direction radiating from the anus, and the pus allowed to escape. It is then syringed out with sterile water or normal salt solution, and after breaking down any dividing walls, so as to convert the abscess into one cavity, it is swabbed out with equal parts of tincture of iodine and carbolic acid, a light ganze drain inserted, and a sterile dressing applied. The patient is not allowed to arise from the table for five or ten minutes after the operation, when he is slowly assisted to his feet, and after a fer minutes in a chair will be able to go about his way.

It is advisable to keep the patient on an absorbable diet for a couple of days and not allow the bowels to move during that time. The wound should be dressed daily, being syringed with plain sterile water or salt solution and lightly packed with gauze. When the author says lightly packed, he means the gauze should be inserted sufficiently firm to keep the wound edges well separated and yet touching against the lining of the cavity 
proper so lightly as not to interfere with its contraction during the healing process.

At the end of the fourth or fifth day in the average case the packing can be dispensed with and a strip of gauze inserted for drainage and merely to keep the wound edges apart. The best protective powder to use to keep the discharge from irritating the surrounding skin is compound stearate of zinc with balsam peru or boric acid.

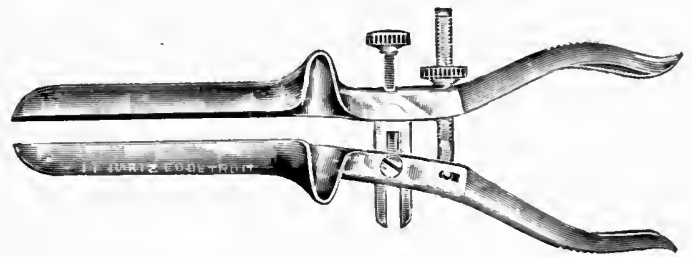

Fig. 69.

DeVilbiss Rectal Speculum.

This instrument is useful in many anal operations, on account of the fact that its blades may be opened parallel to each other and it can be made self-retaining.

When the abscess is of the sub-mucous variety and situated above the internal sphincter, it will be necessary to anesthetize the sphincter according to the technique outlined in Chapter XV. After washing ont the rectum with saturated solution of boracic acid, the patient is placed either in the lithotomy position, if the abscess is situated on the anterior wall; or the lateral position, if located on the posterior or lateral wall. After the parts are washed, shaved and sterilized and the sphincter anesthetized, it is slowly dilated and a Sims retractor inserted at a point opposite the abscess and held by an assistant. In the absence of an assistant, a De Vilbiss rectal speculum (Fig. 69) will answer, as it is self retaining. The mucous membrane covering the abscess is 
injected with a 1-10 of one per cent solution of eucain lactate or sterile water until the tissues are bianched over the entire abscess.

After waiting two minutes for the anesthetic to take effect, the abscess is opened by a longitudinal incision extending from its extreme upper end down to a half inch below its lower extremity. The pus is allowed to drain out, when it is syringed with normal saline solution or sterile water. All dividing walls are broken down so that the abscess is converted into one cavity. It is then swabbed out with $95 \%$ carbolic acid or equal parts of carbolic acid and iodine, and packed with gauze, which should extend out through the anus. In some cases it is advisable to insert a rubber drainage tube about the size of a lead pencil, which tube should also extend an inch outside of the anal canal.

The after-care is similar to that advised for the subcutaneous variety, especial care being taken to see that the abscess is kept healing from the bottom, and that no ramifications form during the healing process. The patient is allowed to be up and about immediately after the operation, and is properly kept up on account of better drainage in the upright position. It is this variety of abscess which if allowed to open without surgical interference forms the blind internal fistula. It is an important thing to remember in this variety of abscess particularly, that the incision should be carried well below the lower extremity of the abscess, so as to allow of good drainage.

Ischio-Rectal Abscess. Ischio-rectal abscesses are the most severe variety which can be treated under local an. esthesia, and not all of these, by any means, are favor. 
able cases. The author would lay down the rule that no abscess of the ischio-rectal region whose upper extremity is over two inches from the anal skin, and whose extent, size and location cannot be definitely outlined by bi-manwal palpation, should be operated on unless under a general anesthetic.

Ischio-rectal abscesses start, grow and extend with great rapidity on account of the loose cellular tissue in which they form, which offer little or no resistance to their spread. They occur at either one side or the other of the rectum, and occasionally surround it. They are formed either from the puncture of the rectal walls by spicules of bone, bristles, or other sharp foreign substances which are swallowed; or from diseased Morgagnian crypts or infection which is earried by the lymphatic system. They have been known to follow operations upon the rectum and anus, or injury through faulty instrumentation in making a rectal examination.

Symptoms.-'The constitutional symptoms are similar to those which accompany the subcutaneous or submucous abscesses with the exception that the pain is more deepseated, the sacral aching more severe, and the symptoms in general approaching more nearly that of a general septic infection. The patient often suffers from chills with a high fever, severe headaches, backache, fetid breath, languor, loss of appetite, and more or less prostration. The pain localizes itself to one side or the other of the rectum unless there is a simmltaneous formation of abscesses on both sides. Defecation is so painful that the patient gives up all attempts at it and frequently is not able to urinate as well. If the abscess has existed longer than 48 hours or so, some redness of the skin will 
be met with, varying in degree according to the nearness to the integument of the location of the abscess.

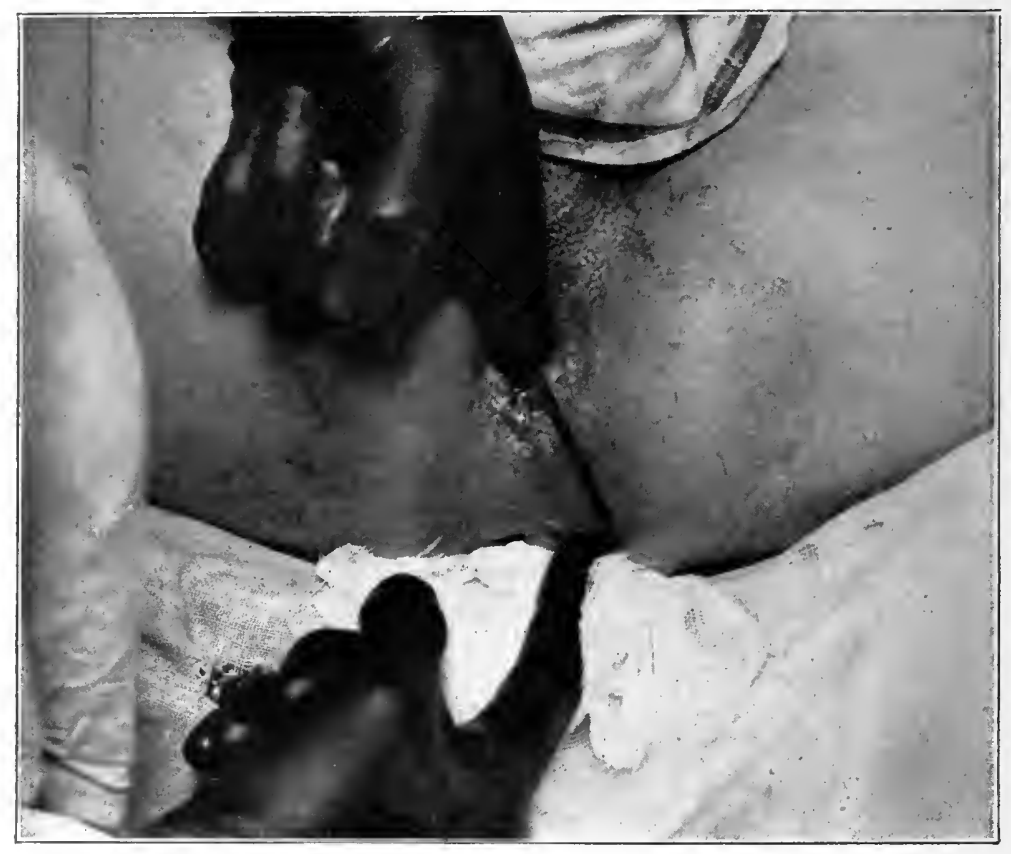

Fig. 70.

Line of Incision for Opening an Ischio-Rectal Abscess. From a photograph of one of the author's cases.

Diagnosis.-Bi-manual rectal palpation with one finger in the rectum and the other hand pressing towards it just outside of the anus (Fig. 70) will disclose a liard elongated mass, often pear-shaped, which is extremely painful, and gives the characteristic doughy or boggy feel of an abscess. A point of fluctuation oftentimes can be made ont at either extremity of the abscess.

The diagnosis is readily made by bi-manual examina. tion. The swelling caused by the abscess may be so great 
that it is practically impossible to introduce the procto scope into the rectum.

Treatment.-After the rectum has been flushed with a saturated solution of boracic acid, the patient is placed in the lithotomy or lateral position, according to the location of the abscess, and the parts washed, shaved and sterilized. The sphincter is anesthetized according to the technique outlined in Chapter $\mathrm{XV}$, and the skin over the abscess, as well as the anal lining membrane, is infiltrated with one-half of one per cent solution of eucain lactate. After the infiltration of the skin, the subcutaneous tissues down to the abscess cavity are injected with one-tenth of one per cent solution of encain lactate, care being taken not to penetrate the abscess carity with the hypodermic needle. The infiltration should be carried well into the lower rectum. A Sims retractor is inserted at a point opposite the abscess and held by an assistant, or the De Vilbiss speculum used, and opened to its fullest extent. With a sharp pointed bistoury an incision is made from the outermost point of the abscess on the skin towards the anus, so that the incision is at right angles to the anal canal. The opening should be made free enough so as to thoronghly drain the abscess eavity, and, if necessary, should be extended through the sphincters into the anus.

Where the abscess cavity can be well exposed by an incision which stops short of the sphincters and there are no ramifications of the cavity, it will not be necessary to enter the rectum, and the author as a rule would caution against making an opening in the rectum unless a communication already exists in the form of a fistula. All trabeculae and partition walls should be proken down 
so that the abscess is converted into one cavity, and it should be well irrigated with saline solution or sterile water. The incision at the lower point of the abscess cavity should be as wide or wider than the cavity itself. After irrigating the cavity, sufficiently gauze soaked in Balsam Peru should be gently inserted so as to keep its walls apart and lightly packed. A dressing is applied and the patient advised to keep in the recumbent position, lying preferably on the side where the abscess is located for 24 lours.

At the end of that time, the packing is removed and abont two-thirds of the quantity of gauze used in the first dressing inserted lightly. At each succeeding daily dressing the amount of gauze is lessened until the abscess cavity has healed up from the bottom. If the granulations become flabby or mnhealthy at any time, they should be touched up with a stick of copper sulphate or a swab moistened with $25 \%$ solution of silver nitrate. The application of pure ichthyol every second or third day, while somewhat painful, is of extreme value in promoting good granulation.

Where it has been found necessary to carry the incision into the rectum and sever the sphincter, care should be taken to arrange the packing in such a way as to prevent the skin or mucous membrane from growing down into the wound, thus preventing the reuniting of the sphincter as the abscess cavity heals.

If this should happen, however, in spite of all precautions, anesthetize the part by the application of a swab soaked in four or five per cent eucain solution for five minutes, keeping up pretty steady pressure on the parts. Then with a pair of sharp pointed scissors curved on 
the flat, trim back all redundant tissue to the surface of the skin or mucous membrane as the case may be.

In the treatment of all suppurative conditions of the ano-rectal region, the author would caution his readers to refrain from attempting to operate on any case in which there is the slightest doubt of his ability to complete the operation under local anesthesia. One must be sure of the size, location and extent of the abscess, and it must be definitely outlined and definitely circumscribed in order to be amenable to treatment under local anesthesia. 


\section{CHAPTER IX.}

\section{FISTULA IN ANO.}

A fistula may be described as a tubular suppurating tract communicating with, or connecting the mucous membrane of the anus or rectum, and the integument contiguous to the anal outlet. Fistulae are of several different varieties, which will be described below. A fistula is the result of an abscess in the anal region which has either been untreated and allowed to rupture of itself, or when opened by the surgeon has, through insufficient, careless or improper after-treatment, been allowed to contract without being made to heal from the bottom. The only exception would be a fistula caused from a puncture wound, either traumatic or surgical.

Fistula in ano is often spoken of as either tubercular or non-tuberenlar. While the author realizes that tuberculosis is a factor to be seriously considered in the discussion of fistula in ano, he will reserve his remarks on this particular variety of fistula until further on in the chapter. What is said regarding fistula in ano below, therefore, must be understood to mean the non-tubercular varieties.

The reason that an abscess degenerates into a fistula in this region rather than completely heal, is due to two factors peculiar to its location. The most important is 
the fact that due to the natural motion of the anus and rectum in the act of expulsion of gas or feces, and the dilation and contraction of the sphincter muscle, the parts are not allowed to remain at rest, and the surfaces are prevented from adhering to each other. Added to this is the important fact that mucus and feces enter the abscess cavity from the rectum and their constant pass. age tends to keep the tract open and prevent healing. A fistula therefore is in reality the tubular contracted remains of an abscess, and is lined by a pyogenic membrane as was its parent abscess.

Varieties.-The variety of a fistula depends on the location and kind of abscess which preceded it. They are divided by some authors into complete and incomplete. A complete fistula being one which gives a direct commumication between the bowel and the surface of the skin, somerhere in the region of the anal opening. An incomplete fistula is one which has an opening either into the bowel alone or one which opens through the integmment only. Complete fistulae (leaving out of consideration those which communicate with other organs, such as the bladder, vagina or urethra) are divided into horse-shoe fistulae and multiple fistulae. The horseshoe fistula is characterized by its having one opening in the anal canal, usually situated between the sphincters at the posterior commissure; and surrounding the anus, communicates with the skin by two openings-one on either side of the anus. A multiple fistula is one which has one or more internal openings and numerous branching channels opening by many external openings on the skin. The incom. plete varieties are known as the blind internal fistulae, which are characterized by the fact that they open into 
154 simple, COMPLETE, Fistula-SYMPTOMS, DiagNosis.

the bowel only, and blind external fistulae, whose only opening is on the skin.

A form of fistula known as the sub-mucous fistula is one which has two openings, both opening on mucous membrane, and is usually found just inside the anal canal. The most common location for the internal opening of a fistula is at the posterior commissure of the anus and between the sphincter muscles. In this chapter only those varieties of fistula which are amenable to treatment under local anesthesia will be discussed, viz., simple complete fistula, blind external, blind internal and sub-mucous. (Fig. 71.)

Simple Complete Fistula. This is the commonest form of fistula met with, and is the remains of a subcutaneous or ischio-rectal abscess, and consists of a straight or slightly curved channel rumning from the anal canal or some point in the rectum a little higher up, to the outside skin-usually opening within one or two inches to one side or the other, and below the anal aperture. The external opening may be at any point on the skin in the vicinity of the anus, but the points mentioned are the most usual sites.

Symptoms.-The symptoms are a sense of irritation or an itching of the anal region, pain during defecation, and the presence of a purulent discharge. If for any reason one of the openings should become plugged up, there is some distension and pain from pressure.

Diagnosis.-The diagnosis of fistula should always be in mind when on examination of a patient a papule is seen on the perineum or bnttocks, from which a drop of pus exudes or can be pressed out. This is the characteristic appearance of the external opening of a fistula. With 


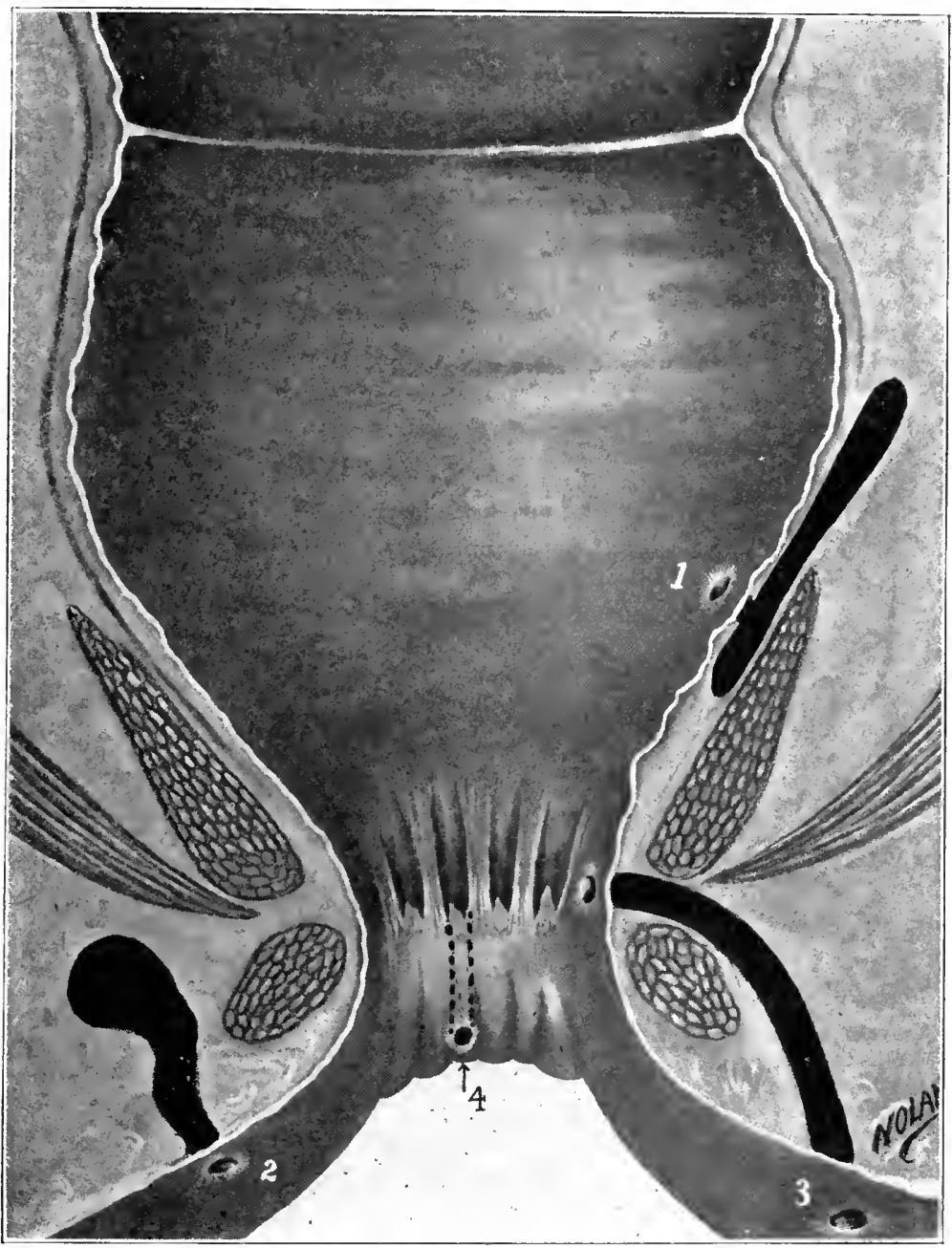

Fig. 71.

Ano-Rectal Fistulae.

1. Blind internal fistula.

2. Blind external fistula.

3. Complete direct fistula.

4. Submucous or submuco-cutaneous fistula. 
the patient in the lateral position and the inder finger of one hand over the external opening, the index finger of the other should be inserted with the palmar surface directed toward the posterior commissure. Often by the

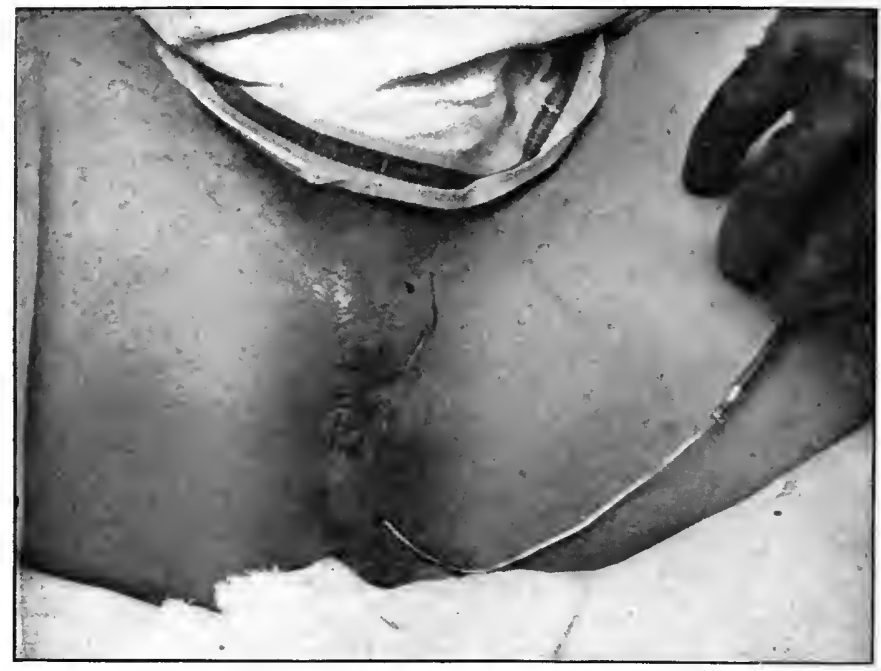

Fig. 72 .

Direct Complete Fistula in Ano.

The probe is seen entering the external or cutaneous opening while directly above it its blunt tipped extremity is seen emerging from the anus.

Photograph of one of author's cases.

pressure with the finger in the rectum a drop of pus will he forced ont through the external opening. By carefully feeling the region between the anal canal and the outside opening, one will often make out the cord-like ieel of the fistulous tract. Oftentimes the internal opening is extremely difficult to find. Upon examination with the author's fenestrated anoscope, or the anoscope with the oblique aperture, a small reddened spot often raised somewhat from the surface will be detected, from which 
pus can be squeezed out. When this point is discovered,

- digital examination will reveal the induration underneath the surface, which discloses the direction of the fistulous tract. If after careful examination of the entire circumference of the anal canal and lower rectum, no internal opening can be detected, the injection into the external opening of peroxide of hydrogen, methylene blue solution, or milk of magnesia or bismuth, will assist one in locating the internal opening by the point of appearance of the solution inside the anus or rectum.

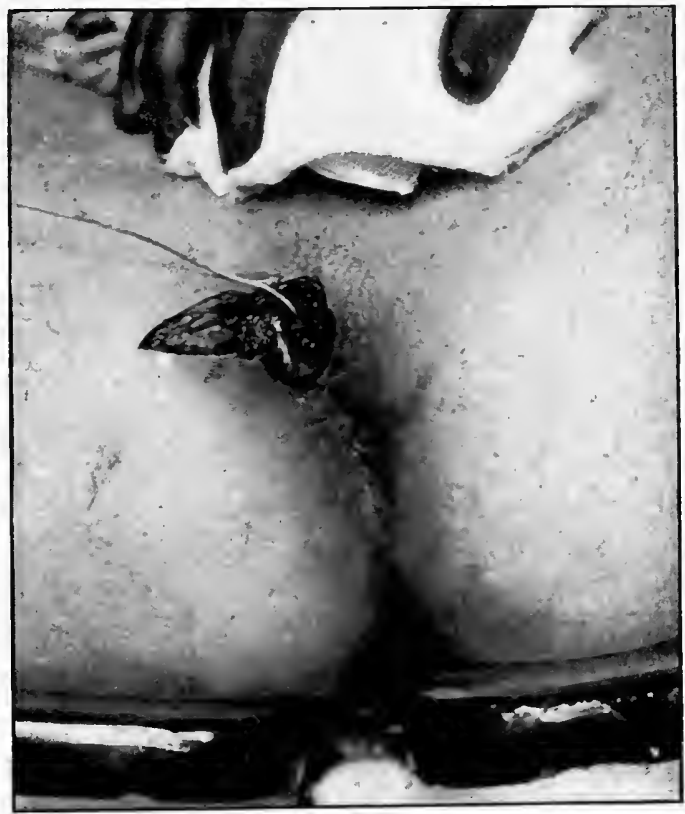

Fig. 73.

Angular Fistulous Tract.

The upper portion of the fistula has been opened and the probe can be seen entering the lower portion. The end of the probe can be seen emerging from the left upper quadrant of the anus.

Photograph from one of author's cases. 
The probe may be used to diagnosticate the presence and direction of a fistulous tract, but in order to be of and value it must be very fine and extremely pliableone made of annealed silver is the best for this purpose. One must be extremely careful in introducing a probe into a fistulous tract, for it is very easy to force it through the walls of the fistula or into the rectum, thus creating a false passage. If the probe does not pass easily it is better to discard it than to use any force in its use. If there is a suspicion that the fistula communicates with the bladder or urethra, the injection of a mild solution of methylene blue (1-5\%) into the organ will settle the question. If such a communication be present the colored solution will exhibit itself at the fistulous opening in very short order.

Treatment.--The treatment of fistula as a general thing is best accomplished under general anesthesia, because many times upon laying open, what appears to be a simple fistulous tract, ramifications and extensions may be found which would necessitate more dissection than is possible to satisfactorily accomplish under local anesthesia. A case of simple, direct fistula, however, which is not tortuous, and in which the external and internal

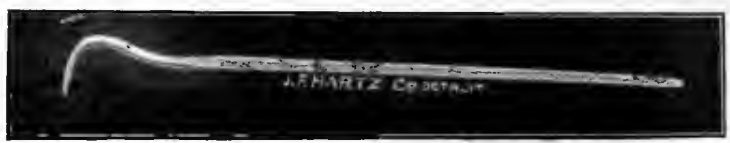

Fig. 74.

Grooved Director.

openings, and the line of communication are made out by the diagnostic methods mentioned above, may be treated under local anesthesia in any one of three ways. 
Incision.-Simple incision will suffice in some cases where the fistula is not deep seated. After the bowels have been washed out with a saturated boracic acid solntion and the area around the anus scrubbed, shaved and sterilized, the sphincter is anesthetized according to the technique outlined in Chapter XV, and the tissues over the fistula injected to the point of blanching with onehalf of 1 per cent solution of eucain. A probe-pointed grooved director is then passed through the fistula from the external to the internal opening, and all the tissues between the director and the surface divided by a curved bistoury passed from withont inward, thus freeing the director and laying open the entire fistula. A pledget of cotton soaked with $4 \%$ solution of eucain is pressed into the incision, and is held firmly against the opened fistulous tract for two or three minutes. It is then removed and the diseased surface lightly curetted with a sharp spoon curette; the incision firmly packed with gauze and an anodyne suppository inserted and a dressing applied.

Unless the direction of the fistulous tract is in a line at right angles to the fibres of the sphincter muscle, it must not be opened by a single straight incision. It is an invariable rule, that any incision which must sever any or all the fibres of the sphincter, should cross it only at right angles (Fig. 75) in order to prevent incontinence afterwards. The incision therefore must be so directed that it never severs the sphincter muscle in an oblique manner. Where the fistula is located just below the skin or mucous membrame and does not involve the sphincter, this rule does not necessary hold good.

Excision.-In some cases it will be found advantageous instead of simply opening the fistulous tract, to excise 
the entire canal. 'This is the most satisfactory operation when it can be successfully carried out, and should be the operation of choice in all straight, uncomplicated fis-

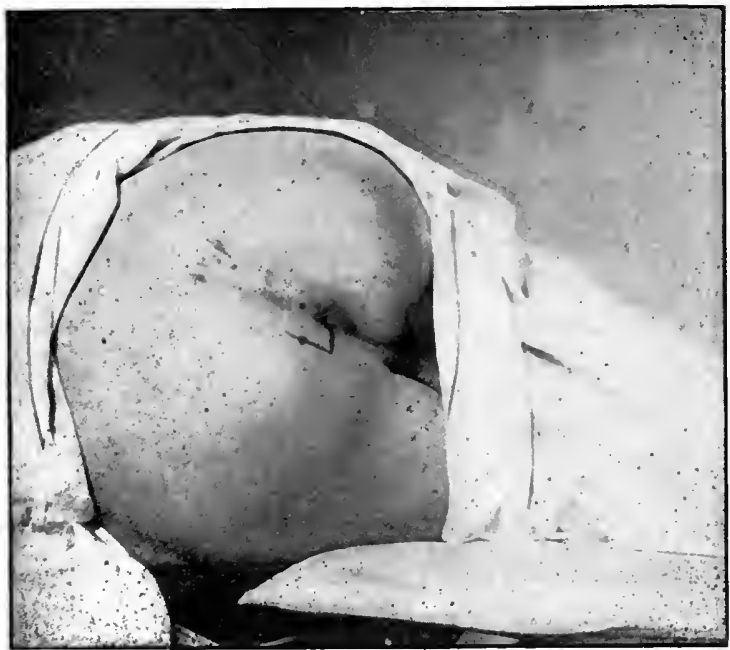

Fig. 75.

Right Angled Incision for Simple Direct Fistula in Ano.

In a simple fistula by which the bowel communicates with the external integument, crossing the external sphincter in an oblique manner, the external sphincter is cut at right angles by the method outlined.

tulae which are situated so that the tissues surromnding the fistula can be sucessfully infiltrated. After the usual preparation of the patient and anestletization and dilatation of the sphincter muscles, the tissues surrounding the fistula are anesthetized. One-half of 1 per cent solution of encain is injected into the skin along the line of incision up to the opening in the anal canal; then the surrounding tissues are distended with one-tenth of 1 per cent solution of encain, care being taken to completely 
surround the fistula on all sides. A grooved director or probe is then inserted and the end, which has been brought through the anal opening, is bent so that it is

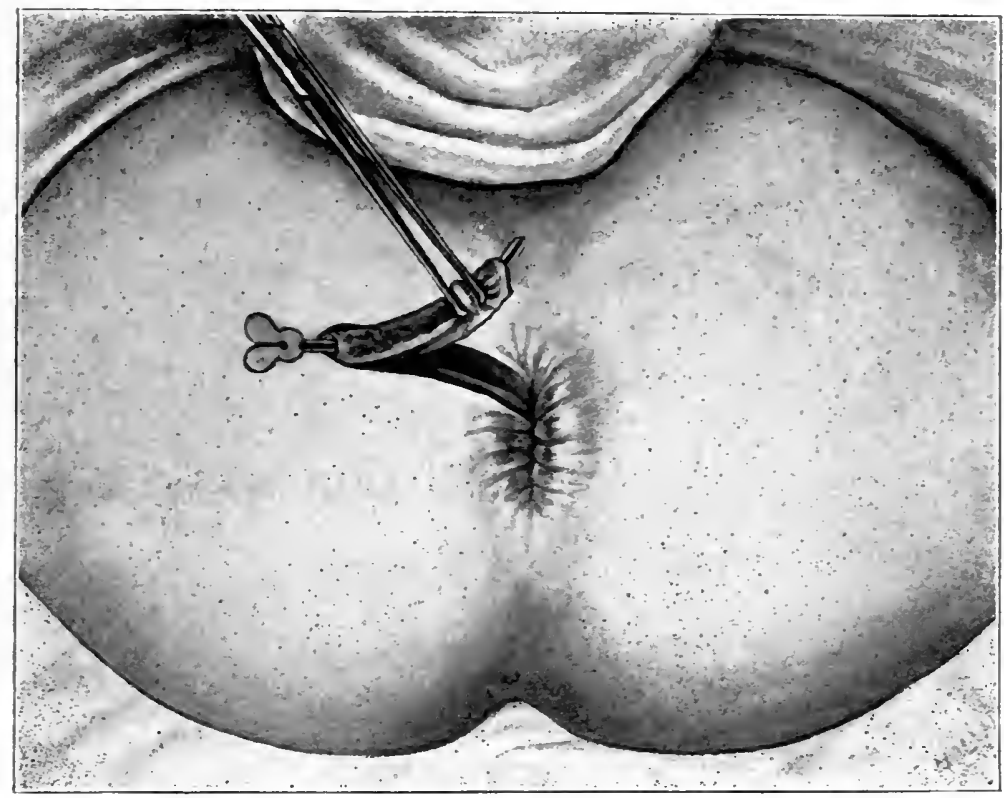

Fig. 76.

Author's Technique for Removing Fistulous Tract in toto.

The lateral incisions are so directed that a V-shaped bed is left, which can occasionally be approximated by sutures.

exposed outside of the anus. This brings the entire tract into view. The skin is then incised the full length of the fistula down to the infiltrated tissues surrounding it, but not through them. (Fig. 76.) The incisions are then carried on either side of the infiltrated fistulous canal in such a way as to free it entirely, and remove it unopened and threaded on the probe. As the incisions are carried around the fistulous tract, they should be brought together in a $\mathrm{V}$ - 
shaped manner beneath it. After the removal of the fistnla, the wound should be packed with ganze, the anodyne suppository inserted and dressing applied.

In the after care following both excision and incision, extreme care must be taken in the daily dressing of the wound to so arrange the packing that it is firm enough to retard too rapid granulation, and yet packed so lightly as to allow the wound to gradually come together. Especial care must be exercised to keep the skin and mucous membrane from dipping in or growing down the sides of the incision. If granulation does not proceed as rapidly as it should, the gauze packing should be soaked with bovinine daily before applying, or pure ichthyol or halsam peru should be applied to the granulating surfaces daily. It is not necessary or advisable to use any of the antiseptic powders in the after treatment of these calses.

The bowels are not allowed to move for three days, after which daily movements are not contra-indicated.

Where it has been necessary to divide the sphincter either in part or in its entirety, there may be some temporary lack of full control of the bowel movements; but as the wound heals up, control is regained so that no fear need be felt on this score. The patient is allowed to be np and around after the first 24 hours, and can pursue his usual oceupation without much discomfort.

Ligature Operations.-In some few cases where either from the desire of the patient that no cutting operation he done, or some other contra-indication, one may occasionally accomplish the cure of a simple direct fistula by means of a ligature either of linen, silk or rubber. The author does not advise the use of the ligature in thes 
cases, as he personally feels that they are never so satisfactory, and certainly not as quick in their results as a clean-cut surgical operation under local anesthesia. If

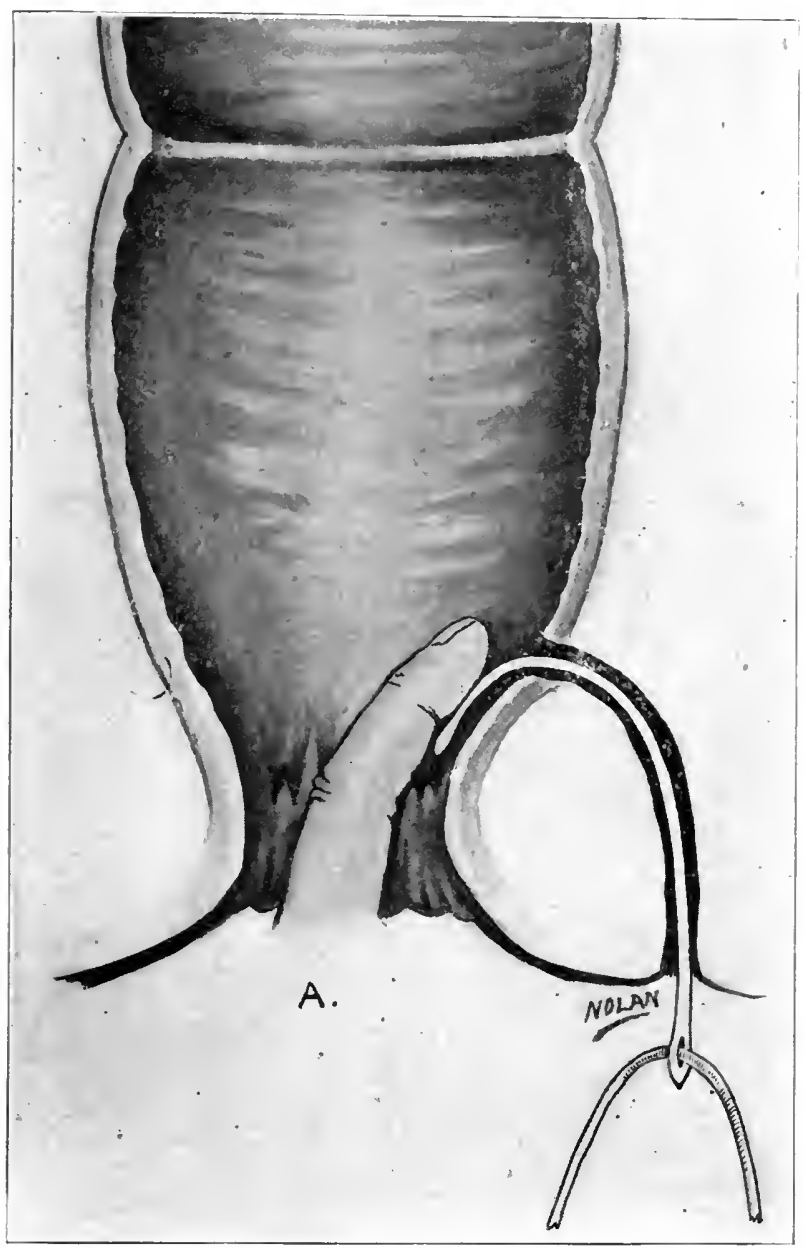

Fig. 77.

A. Technique of Passing Flexible Silver Probe Threaded With Rubber Ligature Through Simple Direct Fistula in Ano. 
the patient must have a ligature operation, the rubber ligature as used by the author in his operation for rectal valvotomy is to be advised, as it is quicker and surer in its results than silk or linen.

The ligature is applied in the following manner: A probe is threaded with the material of choice and it is passed through the fistula from without inward; the point projecting in the rectum is grasped with forceps and is pulled through and outside of the anus. The ligature if silk or linen, is then loosely tied so as not to constrict the parts but lightly surround them and the ends cut off. This leaves a small loop not unlike a seton. This is moved to and fro every day by the patient and in the course of three to six weeks gradually wears through, the fistula healing behind the ligature, as it works through. In some cases, however, this will not prove efficacious.

Where more quick action is desired it is better to use the rubber ligature. It is passed through the fistula, threaded on a probe, in the same manner as the non-elastic ligatures, but when it is fastened with a perforated shot, it is put on the stretch. This causes so much pain and suffering to the patient for the first 12 hours that it is necessary to give repeated doses of anodynes. After this period, however, there is comparatively no pain or discomfort until the ligature sloughs its way through, which occur's in the course of from three days to a week. The suffering caused by the use of this rubber ligature is far more intense than that suffered after one of the radical measures mentioned above, and the author cannot conscientiously recommend it except in those cases where other measures are refused by the patient.

Blind External Fistula. The blind external fistula is 


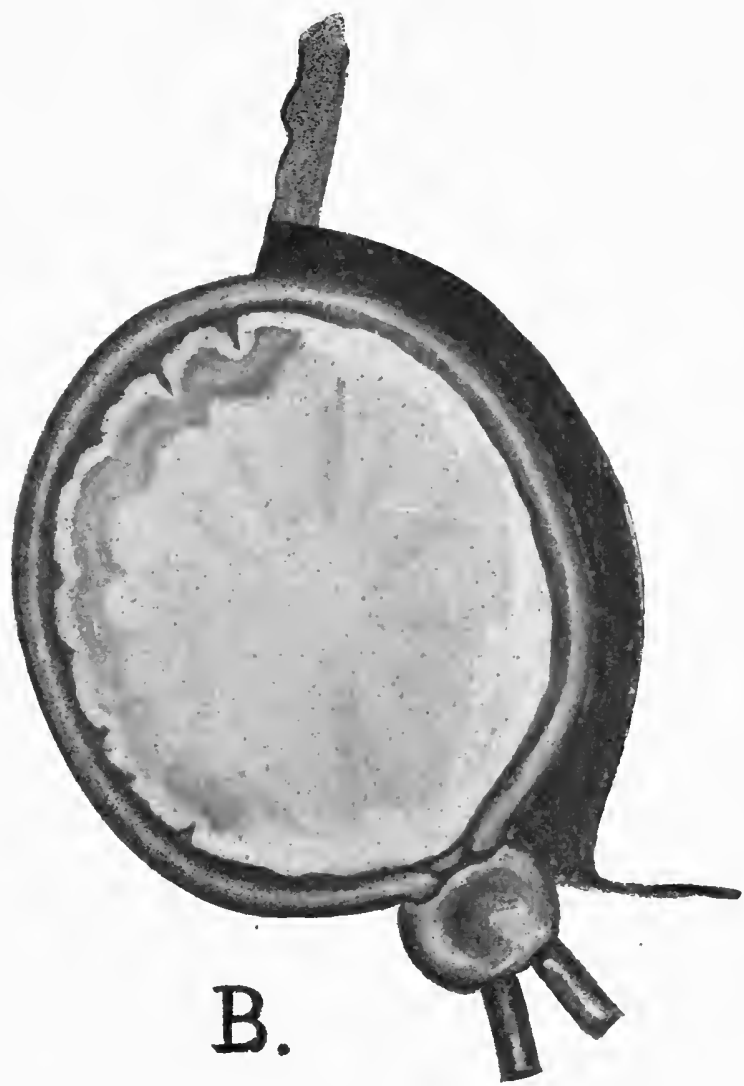

Fig. 78.

B. Showing Method of Constricting the Area Between Fistula, Anal Mucous Membrane and Skin by Means of the Rubber Ligature Drawn Taut and Fastened With a Perforated Shot.

caused by the opening of a peri-anal abscess on the skin surface only. It is characterized by the appearance, after the rupture or opening of an abscess in this region, of a red spot or papule from which pus is discharging. It is accompanied by discomfort to the patient when sitting; pruritus ani. or disagreeable moisture in the region, 
and its diagnosis from complete fistula is made by the method of examination outlined above. In reality it is nothing more or less than a contracted abscess cavity which refuses to heal on account of the action of the sphincter muscle in keeping it open.

The treatment consists in the incision with curetting and packing, or excision of the entire fistulous tract. Some authors advise the converting of an external fistula into a complete fistula and then operating as for complete fistula. The author cannot see the reason or advisability of thus converting a simple abscess cavity into a fistula, and would strongly deprecate any such methods. The anthor does not believe, in fact, knows that it is not necessary to divide the sphincter in order to heal a blind external fistula.

Blind Internal Fistula. This variety of fistula is characterized by its having an opening into the bowel only, and is caused by the rupture into the bowel of a perirectal abscess whose point of least resistance was towards the rectum. They are characterized by their insidions and obscure onset and often go for some time undiagnosed.

Symptoms.-The chief symptom is the appearance of a purulent discharge from the anus. This is accompanied by some smarting, burning, or itching and a feeling of umrest or discomfort in the lower rectum. If there is much involvement of the mucous membrane surrounding this opening there is also a tendency to diarrhoea. When a patient has complained of pain in the rectum persisting for several days, accompanied by heat, throbbing, and rise of temperature; and these symptoms are more or less relieved just previous to the passage of a quantity of pus 
from the anus-the breaking of a sub-mucous or peri-rectal abscess into the rectum should be suspected. The continuance of a purulent discharge off and on for a period of weeks and months, means the existence of a blind internal fistula.

Diagnosis.-With the patient either in the lithotomy or lateral position, a roughened spot with indurated edges is felt on digital examination, usually posteriorly or laterally. Upon stroking or milking the interior of the rectum adjacent to this opening, a purulent discharge will be produced. Upon examination through the anoscope or fenestrated speculum the opening will be seen usinally within the first inch from the anal margin. It will be dark red in color, with edges somewhat raised and the extent of the fistula can be readily determined by examination with a soft silver probe. It is well to bend the probe on itself in the form of a hook, so as to determine the extent of excaration under the mueous membrane of the bowel in the direction of the anus, as not infrequently blind internal abscesses, particularly of the submucous variety, are found with their largest cavity extending down towards the anus. The blind internal fistula is more frequently the result of a submucous abscess than of any other variety, and its chimnel very rarely penetrates the muscular coat of the rectum.

T'reatment.- With the patient either in the lithotomy or lateral position and the external parts washed, shaved and sterilized, the sphineter ani muscle is anesthetized and dilated according to the technique described in Chapter XV. Either a De Vilbiss rectal speculum or the anoscope with the opening on the slant is inserted so as to best expose the opening of the fistula. Its direction and 
extent having been determined, the tissues over the abscess and surrounding it are infiltrated with one-tenth of one per cent solution of eucain. A grooved director is then inserted and the fistula is laid open with a longhandled scalpel or the author's angular rectal scissors. A pledget of absorbent cotton soaked with $4 \%$ solution of eucain is then placed in the abscess cavity and allowed to remain for two or three minutes. 'The interior of the tract is lightly curetted and a strip of sterile gauze inserted for drainage, one end of the gauze being carried outside of the anus. In laying the tract open, the lower extremity should be opened well down to the anus, care being taken to leave no pockets at the lower end. In 24 hours the gauze is removed and a cleansing enema given. The bowel should be allowed to move on the third day, the stools being softened by the administration of liquid albolene, and they should be kept moving regularly each day. Ordinarily these cases will heal without any further attention. It is well, however, to have the patient report every other day for a week or so and to make sure that the cavity is kept healing from the bottom and the granulations healtily.

Submucous Tract. There is a variety of submucous fistula extending usually from the bottom of a crypt of Morgagni which has been called by Wallis, a submucous tract. It consists in nothing more or less than either an unusually small calibered submucous fistula, or a very deep inflamed crypt. It gives rise to an irritating purulent discharge which is very small in amount, but which sometimes is responsible for the production of pruritus ani. In order to determine its presence, it is advisable in those cases where a discharge is noted and no internal 
opening of a blind fistula can be found, to examine with a probe each of the Morgagnian crypts and determine the presence or absence of one of these so-called submucous tracts. If present, it can be slit up with a sharp pointed bistoury after anesthetizing as outlined above. It requires no after-treatment other than examination every other day for three or four days, to make sure that it does not heal over at the surface before it is thoroughly healed underneath.

Submucous or Muco-Cutaneous Fistula. Cripps describes a variety of fistula very similar to the submucous tract which he calls muco-cutaneous fistula. It differs from the variety just described only from the fact that it communicates with the surface through a small opening in one of the anal folds instead of one of the crypts of Morgagni. (Fig. 71,4.)

The treatment of this variety is just the same as that just preceding and need not be described in detail.

The Injection of Bismuth Paste. The use of a mixture of bismuth subnitrate and vaseline in the diagnosis and treatment of fistulous tracts, sinuses and abscess cavities, first brought out by Emil G. Beck, of Chicago, has opened up an interesting field in the non-operative treatment of ano-rectal fistulae. Pennington, in a recent article on the subject in the Lancet-Clinic, December 26 , 1908 , reports 17 cases treated by this method. The paste used by Pennington consisted of bismuth subnitrate 1 part and vaseline 2 parts. To stiffen the paste from 5 to $10 \%$ each of white wax and soft paraffine are added. The technique is as follows:

The patient's bowels are thoroughly washed out and the fistulous tract irrigated as well as possible. An olive 
pointed glass syringe with asbestos packing around the plunger is filled with the mixture, which has previously been sterilized and allowed to cool to a temperature that will not irritate the patient. The point of the springe is pressed well into the main opening of the fistula, if more than one exists, and the paste slowly injected. Should there be an internal opening or communication with the bowel, the finger of the hand not manipulating the syringe is inserted into the rectum to close that opening, thus preventing the paste entering the bowel and aiding in forcing it into all the diverticuli and tortnous tracts. The same precaution is observed where there is more than one external communicating opening. The syringe is not removed as soon as the tracts seem to be filled, but is held firmly in position with slight continuous pressure on the piston. 'The finger' in the rectum is also held in position until the material has hardened, when it may be withdrawn and the syringe removed. A gauze dressing and 'T-bandage are then applied. From one to five injections suffice for the average case, and they should be given either once or twice a week. Pennington's cases required from 2 to 6 weeks for a cure. While he states that this method does not supplant the radical cure of fistula by operation he feels that it should be thoroughly tried in all cases before operative procedures are undertaken, for these reasons:

1. It may cure the case. 2. On account of the aversion of most patients to a surgical operation. 3. On account of the failure now and then of the occasional operator to cure his patient. 4. On account of the fear of the loss of control over the bowels. 5. For its cosmetic effect, there being no scars or irregularities left as after surgi- 
cal operations. 6. Because it is often impossible to tell the extent of the fistula until after the operation is begun. 7. If this method should fail, there always remains the various surgical procedures.

Fistula in Ano in the Tuberculous Patient. The only reason that the discussion of fistula in ano in a tuberculous patient is taken up among these varieties of fistula which can be treated under local anesthesia, is the fact that the tuberculous patient is a very poor subject for general anesthesia. The apparent connection between fistula in ano and tuberculosis is due to the fact of the tubercular patient's resisting powers being away below par. Abscesses in the ano-rectal region tend to fistula formation frequently enough in those individuals who have a normal resisting power; therefore it stands to reason that this should be more so in those suffering from any of the wasting diseases and particularly the most common one, tuberculosis. The tubercular patient's intestinal tract is coustantly flooded with tubercular bacilli and an abscess carity communicating with the gut forms a convenient location for them to locate and propagate. The old idea that the operation for tuberculous fistula has any bad influence on the patient's pulmonary condition is absolutely untenable. As a matter of fact, the local symptoms and inconvenience caused by the fistula make the patient much more irritable and adds to his already overwhelming burden.

Symptoms.-The symptoms are those accompanying fistula in ano as described above, the constitutional srmptoms of tuberculosis being also present.

Diagnosis.-The only point of difference between fistula in ano complicated witl tuberculosis and ordinary 
fistula is the presence in the discharge of the bacillus tuberculosis, and the pink, flabby looking, unhealthy, granulations found around the external opening. There is also a tendency to undermining of the skin edges.

Treatment.--The treatment of a tuberculous fistula is the same as that outlined above for the different var: eties of ordinary fistula in ano, with the exception that when the fistulous tract is laid open after lightly curetting, its inner surface is swabbed with pure lactic or glacial acetic acid. Iodoform or iodosyl gauze is used for packing and dressing on account of the peculiarly antagonistic effect of iodine to the tubercle bacillus. The patient should be encomraged to live an out of door life, and his general bodily nutrition and physical condition looked after the same as any other tubercular patient. 


\section{CHAPTER X.}

\section{HEMORRHOIDS.}

Hemorrhoids, which is the most common disease of the ano-rectal region presenting a pathological change in the tissues, is also the most frequently self-treated condition affecting this region. We see more quack advertisements, more nostrum remedies presented for, more irregular practitioners holding themselves ont to cure hemorrhoids, than any other disease (with the possible exception of venereal disease). In many quarters intelligent people, who would not think of consulting an unethical practitioner for any other condition, will consult the so-called "pile specialist"-who holds himself forth in the daily press-because they believe that members of the regular profession do not treat rectal diseases. It is perfectly astonishing to what an extent this belief is held; in fact, the author is sorry to say that he knows of instances where members of our profession, in good standing, have referred cases of rectal disease to advertising, so-called rectal specialists.

There must be a reason for this, and that reason is the lack of instruction to the medical student on the subject of rectal disease, in the first place; the paucity of such instrnction when given as an incident in the teaching of general surgery; the repugnance with which the average 
practitioner approaches a case requiring rectal examination; the cursory character of such examination; the distaste of the average practitioner for local treatment of the ano-rectal region; the inability to make a correct diagnosis; and the superficial treatment given and the early discharge of the patient by the practitioner, who is anxious to get rid of a ease, which is mpleasant for him to treat-all are responsible for the position which the average general practitioner occupies today in the diagnosis and treatment of rectal diseases.

It is the action of the profession itself which has created the special field of proctology-the anus and rectum being organs peculiar to themselves and being subject to many medical and surgical diseases in the same way as the eye, the ear, the nose, the genital and urinary organs; and call for just as much special medical as for surgical care. The general surgeon knows nothing abont, and cares less for, the medical treatment of these organs; and the general practitioner who is able to treat the medical conditions is not, as a rule, properly equipped to do so. Thus, the proctologist came into existence--a man who, by special study of this particular region of the body, is able to give special care of either a surgical or medical nature, and often both in the same case, as may be required. With his attention directed particularly to this line of work, his operative measures are directed largely along the lines of conservatism. He endeavors to save as much tissue as he can and cut as little as he can, and by intelligent after-care to promote lealing much more near the normal as a rule than does the man who "cuts a fistula and ties a pile" and lets it go at that. That the average general practitioner is fully as capa- 
ble to treat many ano-rectal diseases, as the proctologist; if he has at his hand a practical work ontlining indicated therapentic measures in a plain, simple way; goes withont saying.

The treatment of hemorrhoids in the hands of the practitioner has undergone vast changes since special attention has been directed along this line. In many ways it has been much simplified, and the results have been extremely satisfactory.

Varieties. Hemorrhoids are tumors or swellings produced by pathologic changes in the veins of the anus and rectum, accompanied by more or less infiltration of the surrounding tissues and hypertrophy of the anal skin. They are usually divided into three classes, according to location: External, internal, and externo-internal. The external being those outside of the sphincterial region and covered by integument; the internal being covered with mucous membrane, and whether situated inside of the bowel or prolapsed outside; they are nevertheless internal. An internal hemorrhoid being prolapsed and remaining prolapsed may appear externally, but if it is covered by mucous membrane it is an internal hemorrhoid. The externo-internal variety is a combination of the two preceding, being covered by both mucous membrane and skin. The external, again, are divided into thrombotic, integumentary and varicose.

The thrombotic variety usually appears suddenly; may range in size from a pea to a large grape; is rounded, of a bluish, purplish hue, and extremely painful. It feels much larger to the patient than it really is, and is characterized by its sudden onset. The integumentary variety is a sac or pouch of thickened skin, usually the remains of 


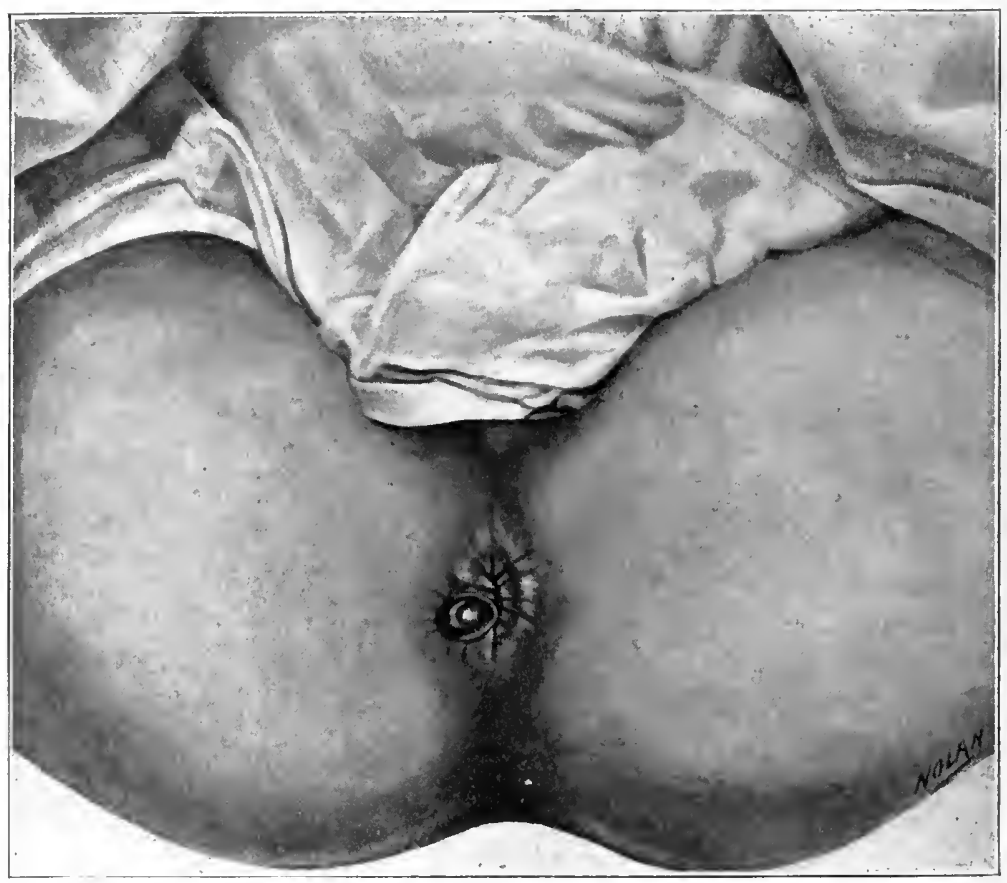

Fig. 79.

Acute External Thrombotic Hemorrhoid.

These are characterized by their sudden onset and are of a bluish or purplish hue.

Drawing from photograph of one of author's cases.

an old acute thrombotic hemorrthoid which has undergone absorption. The varicose variety consists of a collection of small varicose reins covered by skin and situated at or ontside of the anal orifice.

'The intermal variety are divided into the capillary or sramular, and the varicose. The capillary hemorrhoid may not appear as a tumor at all, but simply a circumsoribed reddened area which bleeds upon touch. Where there is an enlargement, it 
looks not unlike a raspberry. Its color is brighter than the varicose variety and it bleeds more freely. The varicose internal hemorrhoid is caused by a varicosity of the veins of the superior hemorrhoidal plexus, the varicose veins, together with the infiltrated skin surrounding them, forming rounded tumors of varying sizes. The internal hemorrhoids may also be divided into pedicled and sessile, either of which variety may protrude through the anus.

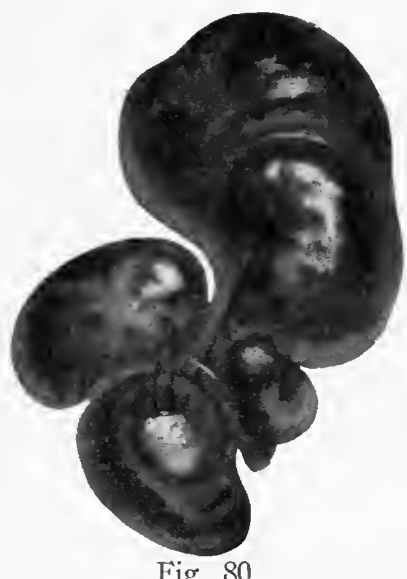

Exiernal Thrombotic Hemorrhoids.

This specimen, remored from one of the author's cases, illustrates the thrombotic nature of the condition. There were four distinct clots present in this case, and they were removed 'n massi.

Causes. A great many different causes have been assigned for hemorrhoids. The principal predisposing cause is the erect position which man assumes, and the lack of valves in the rectal veins, cansing the weight of the column of blood to rest on the veins of the lower rectum and anus. Anything which will abnormally increase this weight or the pressure on the vein wall will, of course, 


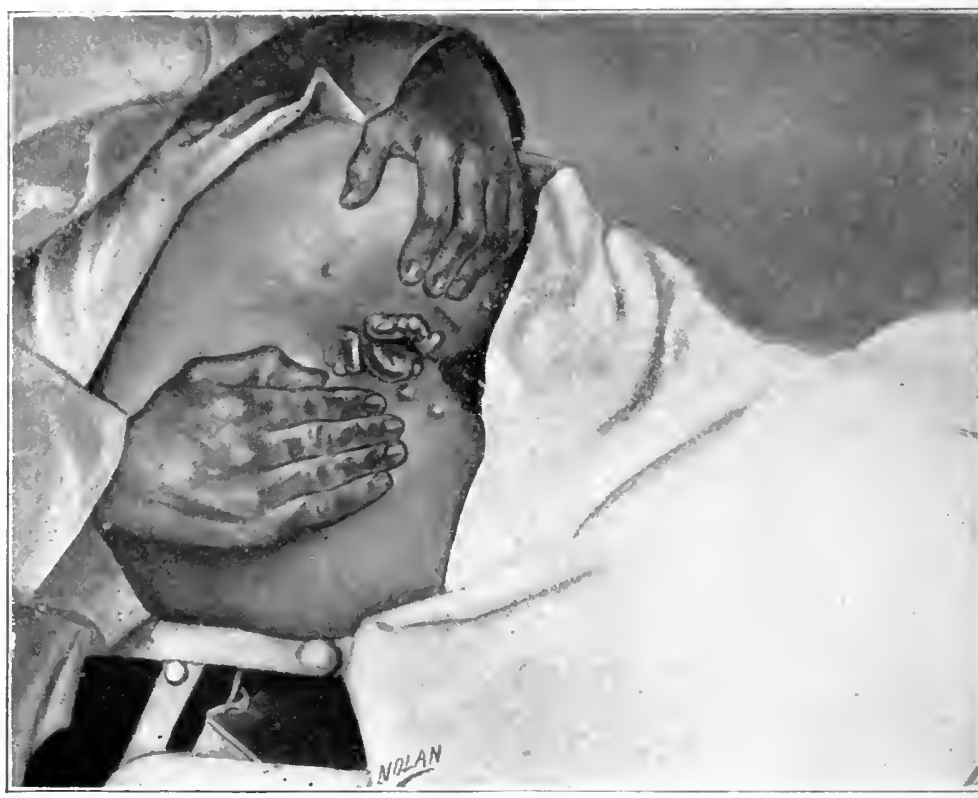

Fig. 81 .

External Cutaneous Hemorrhoids.

Drawn from one of the author's cases suffering from tertiary syphilis.

cause dilatation and enlargement. Constipation is an occasional cause of hemorrhoids. The large, hard stool, as it is passed down through the rectum, pushing the blood ahead of it, and milking the veins, as it were, causing unusual pressure in the lower portions of the hemorrhoidal plexus at the anal canal. A more common cause, however, than constipation is the effort to relieve constipation by means of purgatives; the umnatural straining and the irritating liquid stools being responsible for more cases of hemorrhoids than constipation itself. Over-eating and lack of exercise, or anything which causes a con- 
gestion of the portal circulation are important causative factors in their production. Occupation enters largely into their etiology. Men who are on their feet continually, such as policemen, letter-carriers, pedestrians, railroad men, travelling men-are all peculiarly subject to hemorrhoids. Men are more often treated for hemorrhoids than women, not so much because they are more subject to hemorrhoids, but because women are treated for many gynecological conditions, the relief of which relieves the hemorrhoids. Many women who suffer from hemorrhoids caused by the pressure of the pregnant uterus will be spontaneously cured after delivery.

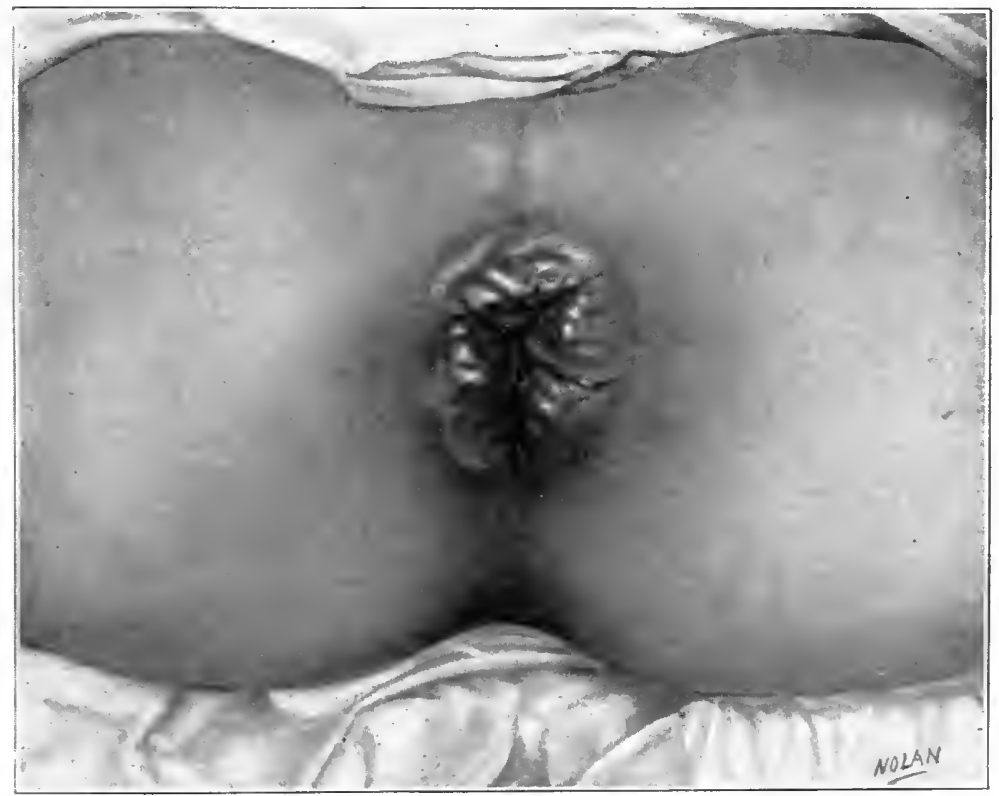

Fig. 82.

Interno-Exteznal Hemorrhoids.

Drawn from one of author's cases. 
The most common cause, however, is in the opinion of the writer, the abuse of the cathartic habit.

Symptoms.-The three principal symptoms associated with internal hemorrhoids are bleeding, pain and prolapse.

The bleeding is of especial interest. Many patients suffering from hemor'hoids scarcely ever, if at all, present the symptom of hemorrhage. In those cases the mucous membrane covering the hemorrhoid (and we are speaking of the internal hemorrhoid at this time) is thick and is not easily ruptured, and the hemorrhoids may pro-

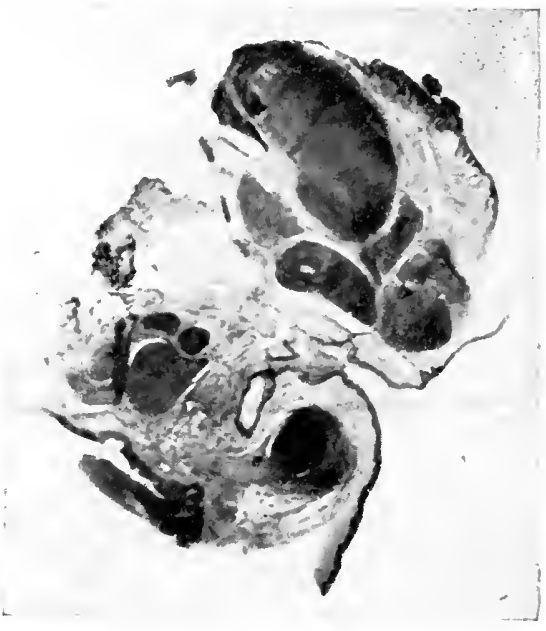

Fig. 83 .

Section of Interno-External Pile.

(Photo-micrograph, $\times 4$.)

Upon the right hand side of the illustration the upper half has a covering of mucous membrane, the lower half a covering of skin, between these there is a sulcus which corresponds with the pectinate line. The upper half is therefore internal pile, the lower, external pile. The structure of the interior of both portions is practically identical-loose areolar tissue with dilated thrombosed veins.

The Rectum: Its Diseases and Developmental Defects By Sir Charles Ball. 
trude without hemorrhage. Where bleeding is observed, it may be very slight, consisting of a few drops following the stool, or is simply noticed on the toilet paper after stool. In other cases it is very profuse, several ounces being lost with each stool, and some patients have become profoundly anemic from this cause alone. I might mention in passing that it is extremely important in every case of anemia to inquire as to whether the patient is suffering from hemorrhoids or not; as not infrequently the rectal hemorrhage will be found to be the cause of the trouble, and its relief will be followed by a prompt return of the normal amount and quality of blood. The writer has observed in anoscopic examination typical arterial spurting from the midst of a hemorrhoidal mass.

Before leaving the subject of bleeding from hemorrhoids the author wishes to utter a word of caution about making a diagnosis of hemorrhoids from the symptom of rectal hemorrhage alone. Many a poor unfortunate has gone to an untimely end because commencing malignant disease was erroneously diagnosed as hemorrhoids because of the symptom of bleeding alone. It makes no difference as to the age of the patient, or whether there is pain present or absent, the symptom of hemorrhage should never be taken for granted as denoting the presence of hemorrhoids; and even where hemorrhoids are observed, no man should be satisfied that he has made a correct diagnosis until he has made a proctoscopic examination (which must include the upper rectum and sigmoid) and the presence of commencing malignant disease has been absolutely excluded.

It is not the intention of the author in this work to cite cases, but he could cite several seen in consultation 


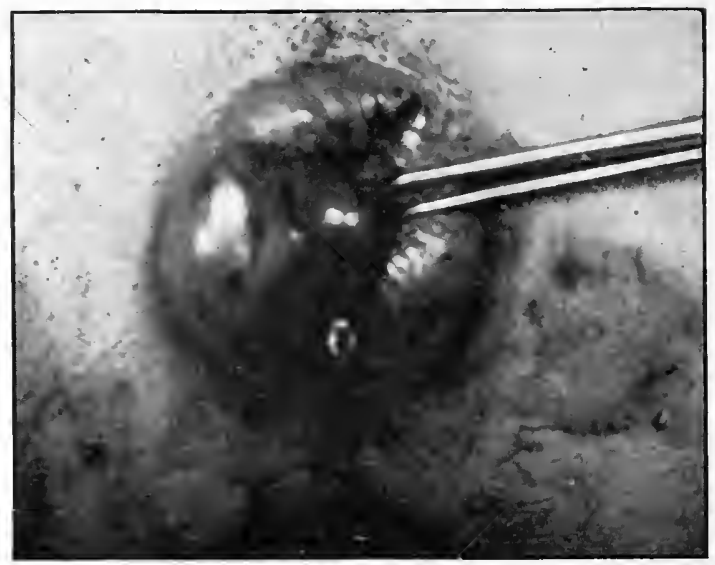

Fig. 84.

Interno-External Hemorrhoid Injected With Anesthetic Solution Ready to Operate.

where the diagnosis of malignant disease was made too late to save the patient's life, because the patient had been allowed to go for months-being treated for hemorrhoids without ever having had a rectal examination made. $\mathrm{He}$ has also seen numerous cases of fissure-in-ano diagnosed as hemorrhoids simply from the appearance of blood following stool.

The pain of internal hemorrhoids is somewhat characteristic, but not pathognomonic. It is more a dull aching sensation accompanied by a feeling of fullness with or without throbbing. It is seldom of an acute nature. The patient complains of a constant sense of weight and dragging in the rectum and in the sacral region, and is usually more or less mentally depressed. Many patients having hemorrhoids suffer from no pains whatever.

The pain accompanying the acute thrombotic pile is 
sudden, lancinating in character, and is accompanied by the appearance of the tumor. The pain soon becomes of an intense, throbbing character, and the relief given upon the incision of the hemorrhoid and removal of the clot, has to be seen or experienced to be appreciated. The other varieties of external hemorrhoids are not accompanied by pain at all, but may be accompanied by considerable prnritus.

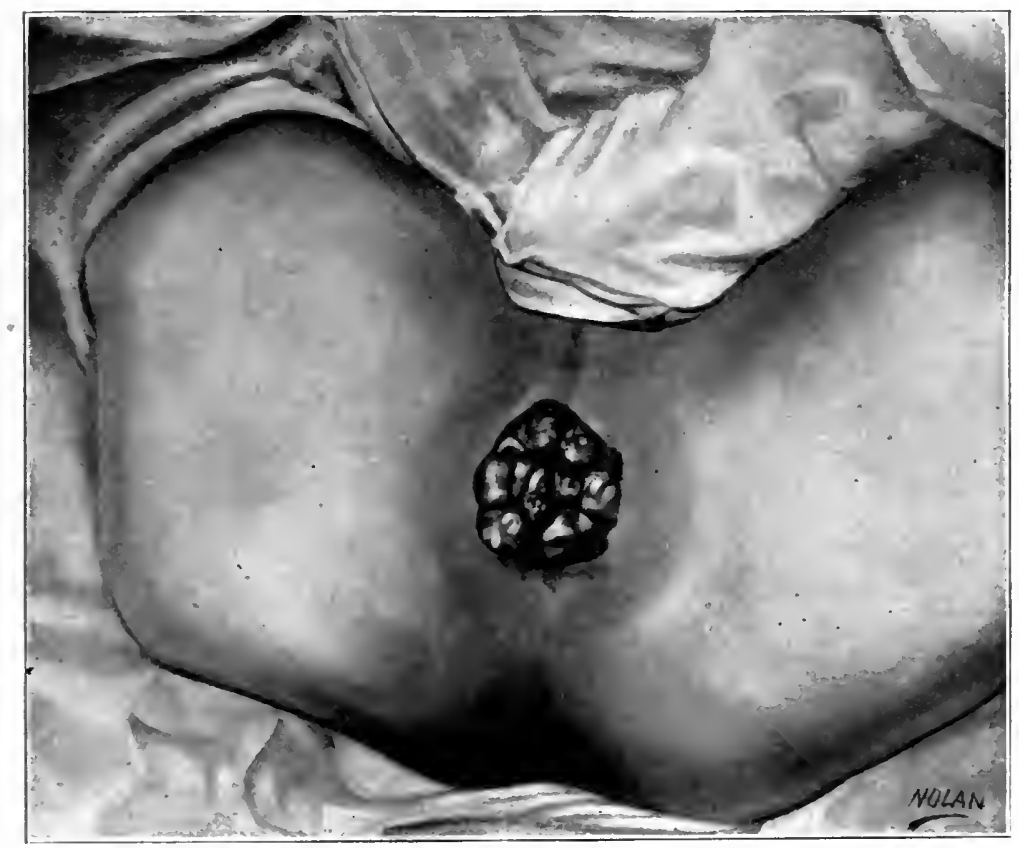

Fig. 85 .

\section{Prolapsing Internal Hemorrhoids.}

Drawn from a photograph of one of the author's cases.

This illustrates the extent to which internal hemorrhoids may prolapse. 'This case was of 20 years' standing and unless the hemorrhoids were prolapsed after stool there was nothing to distinguish the external appearance of the anus in this case from the normal. A case of this severity would of course be suitable for treatment only under general anesthesia. 
In those cases of internal hemorrhoids which prolapse, the prolapse is slight at first, gradually increasing with time. At first the prolapse is replaced readily by the patient after stool, but as time goes on and the prolapse becomes aggravated, it will come down not only with the stors but when the patient is up and about and walking. It finally remains down and can only be replaced when the pritient is lying down, or in the knee-shoulder position, and even when held by pads or retaining devices soon slips out again, when the patient resumes the erect posture and starts to walk.

Diagnosis. One would think that the mention of the diagnosis of hemorrhoids would be superfluous, and that the condition almost diagnoses itself; but it is because of the many unfortunate erroneous diagnoses of other conditions for hemorrhoids, that the author wishes to dwell somewhat upon this point.

In the first place, the average patient, when consulting a physician for suspected hemorrhoidal or other rectal troubles, is asked to stand in front of a table (see Fig. 15) and bend over on it for a "rectal examination," and the physician inserts his index finger as far as the patient will allow him, and that is all; or, he may take a bivalve rectal speculum (Fig. 35), and, if he succeeds in inserting it far enough, will proceed to dilate. Usually before he has gone very far, the patient is off the table and refuses to allow a repetition of the attempt, and that is about as far as the average rectal examination goes.

Now a complete examination (see Chapter III) of not only the rectum, but the lower sigmoidal cavity as well, may be accomplished, practically without pain, and without any dilating speculum. Cylindrical proctoscopes of 
various lengths are used, and through them everything from the anal orifice to the lower sigmoidal cavity can be examined ocularly and an absolutely correct view of the actual condition obtained.

In making an examination for hemorrhoids, first ask yom patient to lie upon the table in either the right or left Sim's position according to the personal preference of the examiner. With the finger protected by a thin rubber finger cot, and properly lubricated, you proceed as follows :

After making a careful inspection of the anus and surrounding tissues, press the point of the finger against the anns, asking the patient to gently bear down as if he were trying to force the finger ont. The palmar surface of the finger should be towards the posterior commissure of the anus. Allow the finger to slowly enter until you have entered the lower rectal cavity; then, slowly turning your finger from side to side, note the conditions. As the finger is being withdrawn, it should be swept around slowly, taking note of the absence or presence of protrusions or abrasions, depressions, elevations-in fact everything which does not feel like the normal velvety smoothness of the anal canal. An important thing to remember is not to try to feel too high. These conditions will all be found within the first two or two and one-half inches, and if one does not insert the finger too far, he will be able to detect a great many things in this small area. One must remember that hemorrhoids of considerable size may not present any musual feeling to the examining finger, because of the pressure of the finger emptying them of blood, and they are more or less effaced at the time. However, one can become sufficiently expert, so 
that he can detect the presence of even these soft elevations, and will note the furrows between them.

If the presence of hemorrhoids is accompanied by a painful fissure, one may not be able to insert the finger without the use of a local anesthetic; the technique of which will be found in Chapter XV. After digital examination has been completed, an anoscope is introduced. the obturator withdrawn, and the patient asked to bear down. This will prolapse hemorrhoias into the instrument, where they can be examined without any difficulty, or lave the patient assume the squatting position and "strain" the hemorrhoids out. Then introduce the proctoscope, and following this the sigmoidoscope. In introducing the proctoscope, however, one must employ the knee-shoulder position. It is in this position only, that satisfactory dilatation of the rectal cavity by pressure of the atmospheric air can be obtained. The folds and creases are all smoothed out and every portion of the rectal lining mucous membrane can be explored with the ere; the size and condition of the rectal valves can be determined, and the presence or absence of ulcers of the rectal wall as well. The sigmoidoscope is entered in this position or the exaggerated lithotomy position, and having an obturator the end of which can be turned at an angle, it can enter the recto-sigmoidal curve withont difficulty.

Thus it will be seen that this entire region can be snccessfully and completely examined without using an instrument which will dilate the sphincter any more than the base of one's index finger. No dilatation is required and no pain is experienced by the patient. Of course, during the examination it may be required to 
swab out or douche out the rectum, all of which can be readily done through the instruments mentioned.

In the differential diagnosis between hemorrhoids and other conditions, which may-simulate some of their symptoms, one might mention first, fissure. Fissure of the anus, which may accompany hemorrhoids, is more often found alone. The pain of fissure is almost diagnostic; it is sharp, cutting, most intense during the passage of a stool. It remains often for several hours following stool, and is accompanied by more or less tenesmus and spasm of the sphincter muscle. The bleeding of fissure always accompanies or follows the stool. It may consist merely of a blood streak on the stool or several drops of blood following the stool, or it may merely be a spot or smear on the toilet paper. The presence of a fissure causes the patient to put off the bowel movement as long as possible, and when he does defecate, the hard fecal masses cause more pain and discomfort than before. Digital examination reveals a fissure with more or less indurated surrounding tissue situated most often at the posterior commissure, or in either the right or left latero-posterior quadrants.

Ulcer of the rectum may be incorrectly diagnosed as hemorrhoids, on account of more or less slight hemorrhage which may accompany it. Ulcer, however, is usually accompanied by diarrhoea; and ocular examination, after eliciting a history of blood in the stool, will settle the diagnosis at once. The same may be said of proctitis; an intensely congested and injected rectal mucous membrane may bleed on stool, but if the conscientious practitioner examines every patient who presents the symptom of blood in the stool, many sources of hemorrhage other 
than piles will be detected and the correct diagnosis made.

Of course the one important thing always to bear in mind when the symptom of hemorrhage is present, is the possibility of the presence of cancer. Cancer, well ad. vanced, may be found in patients who present the appearances of perfect health. When a patient of any age, from childhood up (just as often below 40 as above), presents a history of rectal hemorrhage, which has been preceded by more or less digestive disturbance, including diarrhoea alternating with constipation of several weeks or months standing, with considerable intestinal gaseven though there is no evidence of cachexia or loss of weight; one should be extremely suspicious of malignancy somewhere in the intestinal tract. If the blood is of a dark color, either of a tarry nature or genuine coffee ground, the location of the cancer is higher up. If the blood is fresh, bright red in color and closely follows the stool, and has a more or less nauseating odor accompanying it (an odor which is almost pathognomonic); one should examine very carefully for commencing cancer in the rectum or sigmoid. When one considers that fifty per cent of all cancers occur in the gastro-intestinal tract, and when one realizes that sixteen per cent of all cancers of the digestive tract occur in the rectum or sigmoid, one can readily understand how important it is to examine every case which presents the symptom of rectal hemorrhag'e.

Various protrusions may be mistakenly diagnosed for hemorrhoids. Polypi, which may occur at any age, but occur more often in children, protrude with the stool. They are harder, more fibrous in character than hemorrhoids; and when replaced by the finger, go back into the rectum 
with more or less of a snap, which is somewhat characteristic of this condition. Anoscopic examination shows the polypus to be a small, rounded, hard, fibrous tumor, attached by a pedicle narrower than itself; its attachment C being somewhat higher in the lower rectal cavity than that of a hemorrhoid. Enlarged rectal papillae have been diagnosed as connective tissue piles. The enlarged papilla, however, is small, always triangular, and occasionally long drawn out and somewhat ribbon shaped. It is pinkish in color; does not contain varicose veins. The point or tip is always downward, and it is attached by its base or widest portion. They are situated at the juncture of the anus and rectum, at the lower edges of the crypts of Morgagni.

Venereal warts of large size have been incorrectly diagnosed as external integumentary piles, but close inspection after obtaining a history of discharge from venereal disease, should make the diagnosis evident. Occasionally the protrusion of an anal or peri-anal abscess may simulate an inflamed external hemorrhoid. However, with the finger of one hand in the rectum and the other hand on the protrusion, the site of the abscess cavity can be made out and fluctuation often determined. The sudden onset, accompanied by the intense pain, swelling, redness and rise of temperature always point to abscess formation rather than hemorrhoid.

The protrusion which is often diagnosed as prolapsed hemorrhoids is prolapsus ani or recti. There are three degrees of prolapsus : 1 . Simple eversion of the anal mucous membrane. 2. The descent outside of the rectum of more or less of all coats of the rectum. 3. The descent of the entire rectum with more or less of the sigmoid, which 
may come down to the anal orifice but not necessarily protrude. Prolapsed mucous membrane is differentiated from prolapsed hemorrhoids by its smooth, velvety touch, reddish color, and the absence of varicose veins. It is continuous with the rectal mucous membrane and a distinct sulcus can be made out between the anus and the protrusion in the second and third varieties. In the first variety, careful examination will show it to be mucous membrane continuous with the anal skin. Of course in aggravated cases of prolapsed hemorrhoids more or less prolapsus of the mucous membrane of the anus will accompany it, and the diagnosis is self-evident.

Treatment. The treatment of hemorrhoids we will divide into palliative and radical.

The palliative treatment of hemorrhoids is, however: not a cure, but a relief of acute symptoms for a more or less short period of time. When a patient presents himself suffering from acute prolapsed internal hemorrhoids with more or less strangulation by a reflexly contracted sphincter, the first thing to do is to reduce the prolapse. This is not always as easy as it seems. The reflex contraction of the sphincter on the hemorrhoids shuts off the return blood supply and the hemorrhoid swells so much that it cannot be replaced without anesthesia. If, however, a solution of adrenalin chloride (1-1000) or glycerine be applied by means of compresses, the blood vessels will shrink to such an extent that reduction is often easy. Sometimes the application of cold or alum solutions will cause sufficient shrinking to make reduction easy. Chloretone, one-half of one per cent, or eucaine one to four per cent may be added to these solutions to render them anesthetic. Occasionally applications of 
fluid extract of ergot will help in maintaining the contraction of the vessels after adrenalin has brought them down. An ointment containing adrenalin, one to one thousand, chloretone 20 grains to the ounce in lanolin, injected into the anus after stool and three or four times a day, at regular intervals through a long nozzled collapsible tube; will often assist in allaying an acute attack of hemorrhoids. However, all of these treatments are meres ly palliative, and the hemorrhoid upon the slightest irritation, will enlarge, prolapse and even strangulate again.

Some patients who absolutely refuse more radical measures will submit to cauterization of the hemorrhoid by the thermo-cautery, thus causing a deposition of scar tissue on the surface of the hemorrhoid which by its contraction somewhat lessens its size, and repeated applications of the cautery will reduce the hemorrhoid so that it will not be noticeable for some time. Occasionally such irritants as glacial acetic acid, chromic acid, and 100 per cent solution of nitrate of silver, have been used for a like purpose. The puncture of the hemorrhoidal mass in various places by means of the electric needle, as advocated by Kelsey, has been of some assistance in reducing the size of internal hemorrhoids, but never entirely removes them.

The "injection treatment," which is the treatment usually advocated by most of the irregulars, may be applied in a number of ways. The patient's rectum is cleansed by means of a simple enema, followed by one of the saturated solution of boric acid or some other antiseptic. The hemorrhoid, which should be of the prolapsing variety and one that can be easily extruded into the anoscope, or outside, is injected down to its base with either a mild 
solution containing carbolic acid up to five or ten per cent, if one wishes to cause a nild inflammation and gradual occlusion of the blood vessels by the deposition of fibrous tissue; or by a strong solution of carbolic acid running from 20 per cent to 50 per cent, when one wishes an immediate slough of the hemorrhoidal mass.

When one has but one or two, or not to exceed four, prolapsing hemorrhoids, this method may be applicable, each hemorrhoid being injected at the time. In some cases two or three injections are necessary for each hemorrhoid at intervals of five or six days, but on account of the danger of injecting a blood ressel, and on account of the inability to limit the slough caused by carbolic acid, it is rather an unsafe method, and repeated instances of destruction of large areas of tissue, and sepsis, have been reported.

A rather ingenious method of applying the injection treatment has been adrocated by Franck of Berlin. He employs a 50 per cent solution of carbolic acid in alcohol, and uses it as follows: The hemorrhoid is rendered tense by the application of a wire snare around its base; this is gradually tightened so as to cause the tumor to be slowly congested; the needle is then planted in the center of the mass and several drops of the solution slowly injected. The snare is not remored until the whole mass has undergone thrombosis. Each time it is treated in a like manner and a dressing of some drying powder is applied. In seven or eight days the necrotic tissue will slough off and the granulating surface will be healed in three or four weeks.

'This long period of granulation is another objection to the application of the injection method. With the intro- 
duction of local anesthesia in the radical treatment of rectal disease, the field for the injection method has been greatly encroached upon. It seems to the author much more rational to remove the hemorrhoid by a clean cut surgical incision, under local anesthesia, and have the patient up and about on the second day, and the wound healed in from a week to ten days (this under local anesthesia in office practice), than to use the uncertain, unscientific injection methods. Therefore, the author will confine himself in this chapter to a description of the various methods of operating on hemorrhoids under local anesthesia, as applicable in office practice.

Operative Treatment Under Local Anesthesia. The technique of producing local anesthesia is, briefly, as follows (see Chap. XV.):

Your patient, who has previously had a cleansing and antiseptic enema, is placed upon the table in the Sim's position. A large glass hypodermic syringe is filled with the solution of choice which may be cocaine, encaine. alypin, chloretone or simple sterilized water, as the case may demand. Beta eucaine lactate, any strength varying from one-half to one-tenth of one per cent, is used for anesthetizing the sphincter and is injected in this wise: After sterilizing the parts, a point one-half inch below and posterior to the posterior commissure of the anus is selected. A spray of ethyl chloride or a drop of pure carbolic acid is used to deaden the pain which accompanies the introduction of the needle. With one index finger in the anus, hooking down the sphincter, the needle in the other hand is passed inward, upward and laterally, in a $V$-shaped direction for about three-fourths of an inch, going down into the sphincter muscle, but not through it. From ten 
drops to a drachm of the solution is slowly injected and the needle is retracted to the point of puncture, but not withdrawn; then it is pushed up on the other side in the same manner, keeping about one-half inch away from the anal aperture.

Then three or four minutes are allowed to pass to give the anesthetic time to take effect. Then a vibrator, armed with a cone-shaped vibratode, well lubricated, is pressed against the anus. About three minutes of rapid vibration will dilate the sphincter painlessly to a sufficient caliber to allow the operation to proceed without difficulty. In the absence of the vibrator, one may use the index fingers of both hands, protected by finger cots, and by a gentle massage movement gradually accomplish the same object in a slightly longer period of time.

When the sphincter is dilated, the hemorrhoid is injected, from its base to its apex, with plain sterilized water, or an extremely mild anesthetic solution, such as onetenth of one per cent of eucaine lactate. The particular point to remember is that distension must be carried until the tissues are blanched and the hemorrhoid is in appearance not unlike a Malaga grape.

I very seldom find it necessary to ligate any vessels, as their retraction very soon causes the hemorrhage to cease.

The operation is then proceeded with according to the technique outlined below.

A suppository containing three grains of thymol iodide, two grains of chloretone and two grains of powdered opium is inserted and a dressing applied, but the patient is not allowed to get up from the table for about ten minutes; then is asked to rise slowly and either sit down or lie down as he wishes. I have found that when a pa- 
tient is allowed to get up immediately, some dizziness or faintness is complained of, and I formerly attributed it to the chemical anesthetics injected, until I found that it also occurred in those patients in whom sterile water alone was used as an anesthetic.

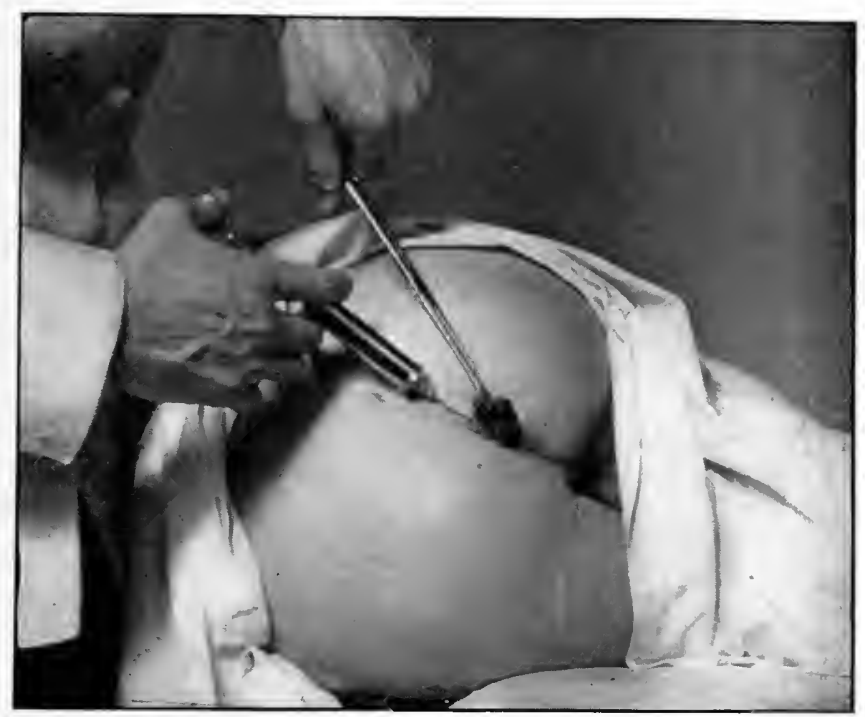

Fig. 86.

Method of Injecting Prolapsing Pedunculated Internal Hemorrhoids.

Excision.-The hemorrhoid having a pedicle is injected at its base with sterile water or weak encain solution-the distension carried to blanching of the tissues, the base transfixed with a double threaded needle (linen suture being used), and the ligature double tied. The hemorrhoid is then cut off, leaving a sufficient stump to prevent slipping of the ligature. Each one is treated in like manner, a suppository of the composition mentioned above inserted, the bowels kept locked up for from three to five days, and 
the patient allowed to be up and around after the first twenty-four hours. The patient is sent home usually in a carriage (occasionally they will walk or take the car), and is advised to lie on either one side or the other for twenty-four hours and then resume his occupation. It is surprising with how little discomfort they are able to get around and how quickly they recover.

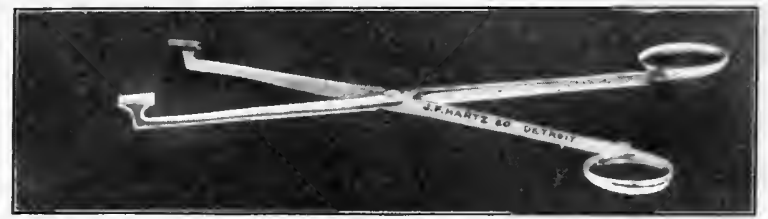

Fig. 87.

Author's Hemorrhoidal Forceps.

Provided with a "Battle Axe" shaped extremity, whose edge is serrated, instead of toothed.

In the author's bloodless operation for hemorrhoids this is a very useful instrument for grasping the tumor without puncturing or lacerating it.

'The hemorrhoid which is sessile or non-pedunculated, is distended in the same manner as above. The most dependent portion is grasped with the author's pile forceps (Fig. 87) or toothed forceps; it is dissected up from its base with either knife or scissors to healthy tissue, care being taken to include in the dissection the vessels which enter the hemorrhoid from above. The upper part of the flap is transfixed and tied off, as is the pedicle in the above variety, when the tumor is cut off with the scissors; others treated in like manner, and the after-treatment is the same as above. It is a very rare thing for the author to have hemorrhage severe enough to require ligation of the vessels. Where there is more or less oozing, a piece of rubber tubing, about four inches long and surrounded 
by gauze, is inserted, and the pressure of the gauze against the raw surface very soon checks oozing. This is removed in anywhere from one to twenty-four hours. Author's Bloodless Operation.-A somewhat simple method is the author's technique for the removal of certain forms of internal hemorrhoids without the profuse hemorrhage with which this operation is usually associated in the minds of most medical practitioners. From the observation that most patients suffering from hemorrlioids of the itnernal variety are more or less anemic from the continued and constant loss of blood, as a result of their hemorrhoidal trouble, I decided to use a technique which would minimize operative hemorrhage and

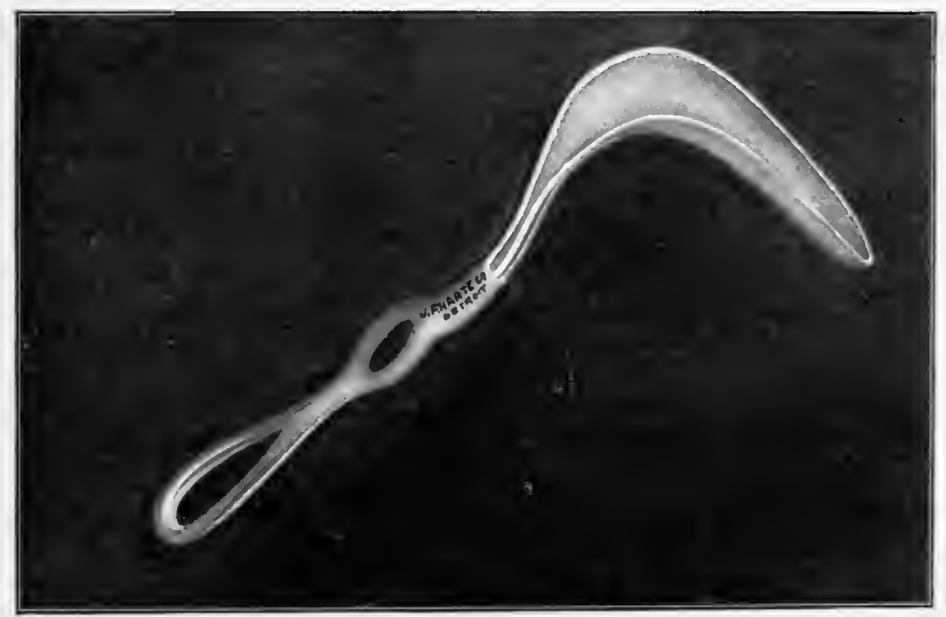

Fig. 88.

Rectal Retractor Modified from Sims' Speculum.

conserve the patient's blood supply. With this aim in view, I have developed and have been using a very simple technique which I present below: 
It is applicable under local as well as general anesthe. sia, and therefore can be used in those weak, run-down patients suffering from any of the wasting diseases, in whom the use of a general anesthetic would be inadvisable, if not positively dangerous. The method is applicable to any variety of internal hemorrhoids and particularly to the pedunculated and prolapsing varieties. Interno-external hemorrhoids can also be treated by this method. Very few instruments are required and in most cases, dilatation of the sphincters is not required. The technique under general anesthesia is much the same as nnder local anesthesia, and inasmuch as local anesthesia is a good deal safer and fully as satisfactory as general anesthesia for this work; the author will describe the operation as performed by him under local anesthesia.

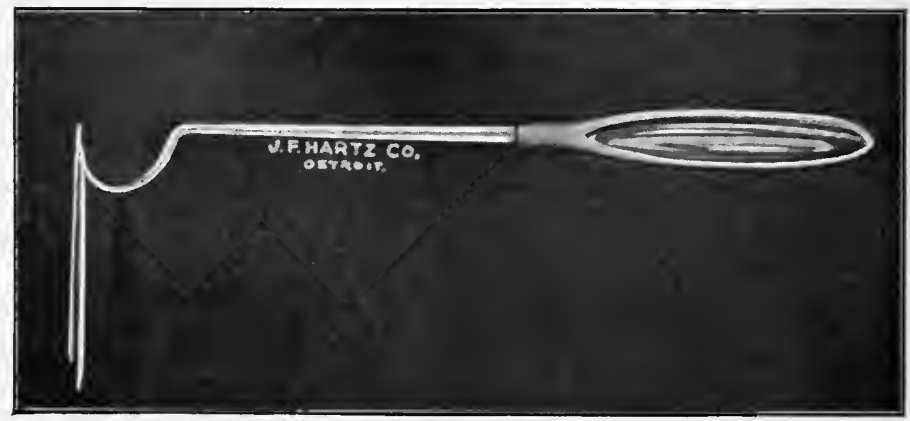

Fig. 89.

\section{Author's Blunt Pointed Ligature Carrier.}

This instrument is very useful in the author's bloodless operation for hemorrhoids, for passing the ligature under the blood vessels of the hemorrhoid; its blunt extremity preventing the puncture or injury of the vessels.

The instruments required are a rectal retractor (Fig. 88), or Sim's speculum; the author's blunt pointed 
ligature carrier (Fig. 89), the author's pile forceps (Fig. 87), scalpel, sharp pointed scissors curved on the flat (Fig. 54), aseptic hypodermic syringe with sharp needle and sterile cat-gut. The patient is given one-fourth grain of morphine about twenty minutes before the operation is performed; the bowels are washed out with a soap suds enema, followed by a boracic acid enema. He is then placed on the operating table in the Sim's lateral position; the skin around the anus is scrubbed, shaved, and sterilized. The sphincters are then anesthetized by the injection of 20 to 30 minims of one-half

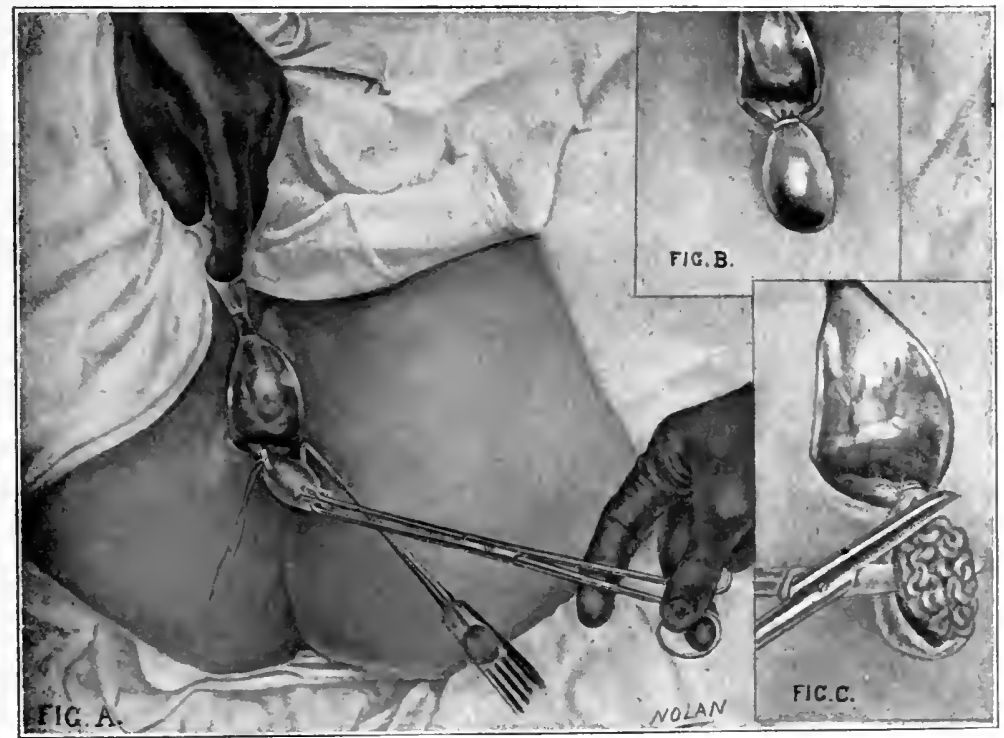

Fig. 90 .

Technique of Author's Bloodless Operation for Internal Hemorrhoids. $A$. Method of inserting ligature carrier threaded with catgut.

$B$. Showing ligature tied, thus constricting the blood vessels supplying the hemorrhoid.

C. Removal of the hemorrhoidal mass without sacrificing the mucous membrane. 
of one per cent beta-encain lactate solution which has been sterilized by boiling, according to the technique described above.

When dilatation has been accomplished, the most dependent hemorrhoid is injected with one-tenth of one per cent solution of encaine lactate or sterile water, and the distension carried until the tissues are blanched. Anesthesia is then complete. The lower extremity of the hemorrhoid is then grasped with the author's pile forceps and pulled down so that it is on the stretch. The bluntpointed ligature carrier, threaded with No. 2 cat-gut, is passed in through the mucous membrane on one side, down to the base of the hemorrhoid and around to the opposite side, in such a manner as to include the upper half of the mucous membrane covering the pile, and the blood vessels underneath, but not encircling the entire hemorrhoid as in ligating a pedicle (Fig. 90-A). This ligature should be placed just at the juncture of the pile and the healthy mucous membrane of the rectum. It is then firmly tied (Fig. 90-B), and it will be found that the blood supply of the pile has been included in the ligature and shut off. The piles at either side are dealt with in like manner and lastly the upper ones. A suppository containing :

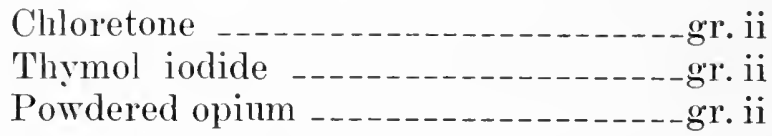

is inserted, the patient keeping in the recumbent position for ten minutes, and then allowed to rise from the table and go to his bed. There will be considerable swelling during the first 24 hours, but this with its accompanying 
pain, can be relieved by the application of hourly compresses soaked in the following solution:

I

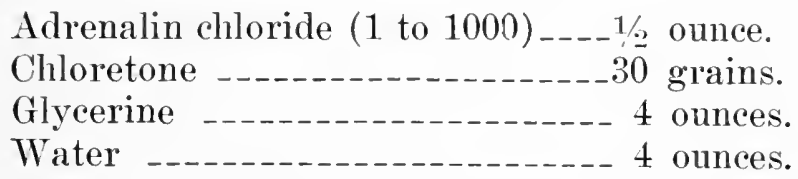

This swelling subsides in from two to four days and the pile gradually shrinks until at the end of four weeks, there is nothing left but a little hard "nub" of connective tissue which can be snipped off painlessly with the scissors at any time. This, which is the simplest form of technique, is applicable to those desperate cases of anemia where the continual loss of blood from the hemorrhoids is greater than the patient's blood production. It can be done in from ten to fifteen minutes' time, and involves the least expenditure of nerve endurance and suffering of the patient. In cases where the necessity for laste is not quite so imperative, I use the following modification of the technique:

After the hemorrhoid is anesthetized as above, and the ligature applied in the same manner, the pile is grasped in the author's pile forceps and an incision made in its longtitudinal axis, starting about one-quarter of an inch from the ligature and extending down to its distal extremity, then with the curved scissors, the blood vessels and connective tissue which make up the body of the pile, are dissected out en masse (Fig. 90-C) and cut off about one-quarter of an inch from the ligature. The wound is left open to heal by granulation, which it does in a very few days. This dispenses with the hemorrhoid at once 
and does away with the swelling, pain, and discomfort which necessarily follows the preceding technique.

In cases where we have pedunculated, prolapsing hem-. orrhoids, it is not necessary to dilate the sphineter or use the speculum. Following an enema, the patient is asked to strain while in the squatting position or lying on his side, while the operator is everting and pressing back the sphincter muscles by pressure just outside of the outer margins of the external sphincters. The pile which is prolapsed by this method is injected with the weaker anesthetic solution. Its pedicle is transfixed with the blunt ligature carrier double threaded with cat-gut and tied off in two sections. The pile is then cut away onequarter of an inch from the ligature, and the stump cauterized with 95 per cent carbolic acid. The other pedunculated hemorrhoids are treated in like manner, the analgestic supository inserted, and the operation is completed.

The after care is very simple, the bowels being confined for from three to five days. A drachm of compound licorice powder at night followed by a six to ten ounce oil enema in the morning, will produce an easy and satisfactory movement at the end of that time. A teaspoonful of liquid albolene before each meal will keep the bowels in good order and daily soft movements will follow. The only dressing required is some drying and protective powder such as compound stearate of zinc, which should be applied sufficiently often to keep the parts protected. Some of the many advantages of this method are as follows : 
1. The technique is simplicity itself.

2. It is applicable under local anesthesia.

3. It takes a shorter time than any other method which suceessfully disposes of the hemorrhoid.

4. It is surer, safer, and quicker than the "injection method," and is applicable in every case where the injection method can be used, as well as in other varieties of hemorrhoids where the injection is contra-indicated.

5. It should be the method of choice in all patients suffering from anemia, tuberculosis, hemophilia, and in pregnaney; because of all the foregoing reasons, and the fact that it doesn't involve the loss of blood. 'The principle of tying before cutting reduces the waste of blood to a minimum, and makes for rapid convalescence.

6. There being no confinement to bed after the first 24 hours, the patient may be up and about, going out of doors, getting fresh air, sunlight and exercise, which are nature's best curative agents in convalescence after any operation or disease, and of the greatest value to patients suffering from any of the wasting diseases mentioned above.

Submucous Excision.-In the sessile variety, another way of treating these is simply to make an incision in the longitudinal axis of the bowel through the center of the mass, and then by the use of the author's angular rectal scissors (Fig. 48) to macerate and destroy the blood vessels beneath the mucous membrane on either side of the incision. The blood supply being destroyed and the macerated tissue cleaned out with a curette, the wound is allowed to heal without suture, and usually does so in four or five days. Of course this method is accompained by some hemorrliage, but never severe 
enough, however, to require ligature. The after-treatment is the same as in the other varieties.

The clamp and cautery operation is not applicable, of course, under local anesthesia, and I mention it merely to condemn it. I do not believe that the use of a red-hot iron in a cavity lined with mucous membrane is rational, and while I am aware that many surgeons have used it with many successful results, I have seen strictures following its use which were caused by the overgrowth of scar tissue-which is more prone to follow a burn than any other form of wound. A clean cut surgical incision, to my mind, is more rational and is not followed by the extensive sloughing or the extensive cicatrix. Crushing the hemorrhoid with the angiotribe has also been used by some operators, and offers the objection that it destroys too much mucous membrane and is followed by a more or less chronic granulating surface taking weeks to heal.

The Whitehead operation is, in the author's opinion, very seldom, if ever, indicated.

Other methods of disposing of large redundant hemorrhoidal masses by means of elliptical flaps, longitudinal incisions, and plastic work, are used to obviate the necessity of doing any operation, which is almost certain to be followed by sepsis, retraction of flaps and subsequent cicatricial contraction; and the writer has yet to see a case of hemorrhoids accompanied by prolapse, so severe that he has not been able to remedy it withont sacrificing the normal contour of the anus. These last varieties have been mentioned simply because no chapter on the treatment of hemorrhoids will be complete without their recognition by some mention at least. 
The acute thrombotic variety (Fig. 79) is peculiarly amenable to treatment under local anesthesia. On account of its sudden onset and the acute suffering which it produces, the patient will present himself for treatment within a very few hours after its onset. Examination in the lateral position shows a rounded, bluish or purplish tnmor varying in size from that of a pea to a large grape, located just at the anal margin usually on one side. It usually occurs singly. After the usual preparation, the hemorrhoid is injected from its outermost aspect with 10 or 12 drops of one-half of one per cent solution of eucain lactate-the injection being carried just underneath the skin or mucous membrane, and not down into the pile. After allowing a minute or two for the anestletic to take effect, an incision is made through the skin and down to the clot, parallel to the long axis of the anus and extending for abont a quarter of an inch into the skin beyond the tumor. The tissues around the tumor and below it are injected with one-tenth of one per cent solution of eucain, when it is dissected out by means of a small toothed forcep and the scissors curved mpon the flat. After the clot (Fig. 80) is removed, look carefully into the wound to see whether a second clot has formed below, and if so, it must be removed at the same time. The edges of the wound are trimmed back in an elliptical manner, so as to leave a gaping wound, which will heal by granulation from the bottom, without any possibility of the edges of the wound turning in and retarding its healing. A onehalf inch strip of ehloretonized tape or gauze is lightly inserted into the wound and a sterile dressing applied. This gauze is removed in 24 hours, when it will not be found necessary, as a general rule, to re-pack the wound. 
It should be seen and dressed daily, and some mild antiseptic powder applied such as thymol-iodide, boric acid, boro-chloretone, stearate of zinc, or acetanilid. The patient after this operation, experiences a keen sense of relief almost from the start from the relief of the tension caused by the thrombotic mass.

The removal of external hemorrhoids of the integumentary (Fig. 52) variety is very easily accomplished under local anesthesia. After the parts are prepared, shaved and sterilized with the patient placed in the left lateral or lithotomy position, the most dependent pile is selected, the point of puncture touched with a drop of pure carbolic

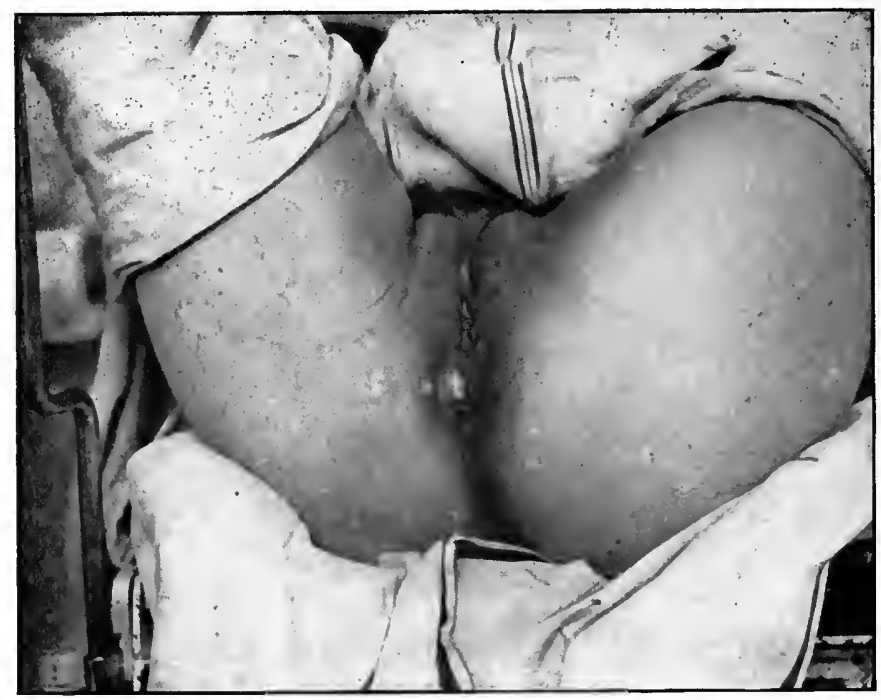

Fig. 91.

\section{Distension of Exterrial Hemorrhoids With Sterile Water.}

This photograph of one of the author's cases shows the amount of distension necessary to produce anesthesia with plain sterile water. This is taken from the same case as figure 52 and comparison of the two will be of interest. 
acid or sprayed with ethyl chloride until the tissues are blanched, when the spray is removed, and as soon as they have regained their natural color the injection is made. As in all operations involving the skin, the first injection should be of one-half of one per cent solution of eucain lactate, care being taken to inject the first ten or fifteen drops just underneath the skin along the line of the proposed incision so as to form a wheal or welt. An incision is then made on a line radiating at right angles from the anal orifice to the distal extremity of the tumor, then the subcutaneous tissues are infiltrated with one-tenth of one per cent encain solution or one-laalf of one per cent solution of chloretone, or sterile water. The hemorrhoidal mass is then seized with the author's hemorrhoidal forcepts and removed with a flat pair of scissors. The skin edges are trimmed back on either side in the shape of an ellipse, so as to include all of the redundant tissue which forms the covering of the pile. One must be cautious about not cutting away too much skin. The distension with the anesthetic solution somewhat distorts and distends the skin, and the infiltration extends beyond the part to be removed, making it appear much larger and extensive than it in reality is (Fig. 91). It is a wise plan, therefore, to carefully mark out, before proceeding to operate, the extent of the proposed incision by means of a small swab moistened with tincture of iodine. Each hemorrhoid is treated in like manner, working from below upwards, and the wounds lightly packed with chloretonized gauze and the wound allowed to heal by granulation. There is no objection to putting a couple of silk-worm stitches in each wound, if desired, but the author has found healing fully as satisfactory without stitching and 
the time of the operation is materially lessened, which is an important factor in all work under local anesthesia.

The after-care is similar to that outlined in the treatment of acute thrombotic hemorrhoids. The healing following operation for external hemorrhoids should be complete in a week or ten days.

During the healing process, the patient should be required to use an infllated air cushion, or pillow, when sitting, and to lie upon either side rather than upon the back. As has been stated above in the treatment of internal hemorrhoids, it is wise to put the patient upon a light diet, consisting of meat, broths and strained vegetable soups, with the addition of eggs or gelatins for the first three or four days. The bowels should be confined and not allowed to move until the fourth day, when, by means of a dose of licorice powder (one to three drachms) given the night before, and a ten ounce oil enema, the bowels should be moved. The movements thereafter should be kept soft by the administration of drachm doses of refined petroleum oil (liquid albolene), four or five times daily, and the diet gradually increased. After the first movement, daily evacuations of the bowels should be procured.

Where, on account of the number and redundancy of external hemorrhoids, the operation for their removal under local anesthesia would be too extensive or involve too much time if attempted at one sitting, the work may be divided; half being taken care of at one time, and the other half after an interval of two or more weeks. The author would not advise the removal of more than three or four external hemorrhoids at one operation. It is very rare, however, to find more than this number as a general rule. 


\section{CHAPTER XI.}

\section{RECTAL POL PI-HYPERTROPHIED ANAL PAPILLAE_CRYPTITIS.}

A polypus is a non-malignant tumor, whose chief characteristic is its attachment to the rectal wall by a pedicle, which is always narrower than the tumor (Fig. 92). It occurs more often in children than in adults. Polypi may be found singly or in such large numbers as to entirely fill the rectal cavity, and will be found complicating fissure in ano, hemorrhoids, prolapsus, and other rectal diseases.

The usual location of a polypus is in the lower end of the rectal canal from one to two inches from the anal opening. 'Rarely cases have been seen in which the polypus has been found attached by a pedicle four or five inches long as high as the recto-sigmoidal juncture.

The types of polypi most commonly met with are either the soft myxomatous or adenomatous variety, and the hard fibroid polypus. In appearance, the soft granular polypus resembles a raspberry, and bleeds readily at the touch. The fibroid variety is hard, rounded and lighter in color than the normal rectal mucous membrane.

Symptoms.-The usual symptoms outside of the appearance of the polypi itself, are the passage of blood, mucous, and straining efforts after stool ; the paitient complaining of a feeling, as if more fecal matter were 
rectum, hut it was impossible to evacuate it.

Diagnosis. - The diagnosis is very simple, as they are often discovered protruding from the anus. A pecu-

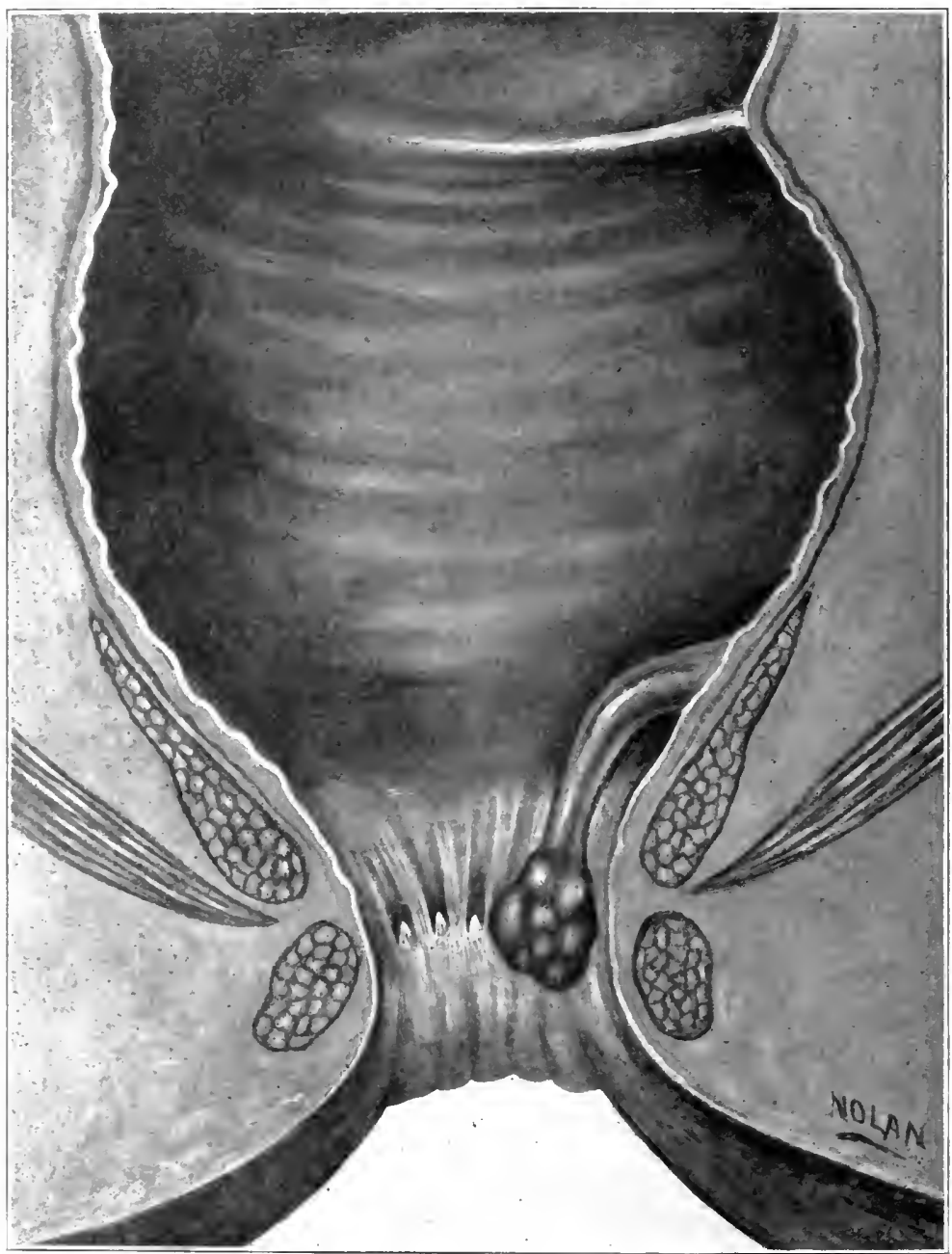

Fig. 92.

Rectal Polypus. 
liar characteristic of polypi is the snapping sensation which they give to the finger as they are returned to the rectum. On making a digital examination, with the patient in the lateral position, one should insert the finger as high as possible, and then sweep it from side to side, completely encircling the rectum on its withdrawal, when the polypi will be discovered, usually just above the internal sphincter. As the finger is withdrawn, the polypi can often be brought with it, outside of the sphincter. By means of proctoscopic examination, polypi situated higher in the rectum may be discovered.

Treatment.-In the treatment of polypi, local anesthesia is often not necessary. They can be snared off with ease through the anoscope or proctoscope with little or no pain. Where the polypus is situated low, so that it can be protruded through the anus, the pedicle may be infiltrated with one-tenth of one per cent solution of eucain or chloretone, transfixed with a double threaded needle, and the pedicle tied off in two sections with a double ligature. The polypus is then snipped off with the scissors, leaving as little stump as is possible. It is practically never necessary to anesthetize the sphincter and no after-treatment is required.

Hypertrophy of the Anal Papillae. - In devoting some space to the anal papillae, the author has done so with the view of bringing before the profession a condition which is practically never recognized by the general practitioner; and usually overlooked by the general surgeon, who includes rectal surgery as an incident in his practice. It is one of the many minor conditions which originate in the anal canal which, while never causing such serious symptoms as to endanger health or 
life, or to cause such great suffering as to incapacitate the patient from his daily occupation; nevertheless, is of no small interest to the medical practitioner because of the amount of discomfort it causes.

This may only amount to an uneasiness, but the hypertrophied anal papilla is often responsible for symptoms ridiculously out of proportion to the size and severity of the lesion.

Many irregular practitioners who hold themselves out as "rectal specialists," have made great capital out of the anal papillae and have attributed to them the cansation of nearly every disease in the calendar. As a result, many of the profession have gone to the other extreme, and have completely ignored the existence of what has been proved to be definite diseased conditions of definite anatomical entities.

When a patient complaining of indefinite rectal or anal symptoms consults his physician, too often he is dismissed with some proprietary ointment, without any effort being made to locate the cause of the trouble. The special study of the Rectum, with its allied organs, the Anus and the Sigmoid, has brought to view many interesting conditions which have been overlooked in the past; and it is with the view of clearing up some of the obscure and indefinable symptoms which originate in the region of the anus, that the author is devoting this space to Hypertrophy of the Anal Papillae.

It is in the anal canal, where most of the pathological conditions which cause pain and suffering, and reflexes without number originate. Nature has been unusually lavish in her sensory nerve supply to these organs, and lesions in this region produce referred disturbances in 
many other and remote organs. When one considers that the anal canal measures from two-thirds to an inch and a quarter in length and its circumference about one and one-quarter inches in the contracted condition; one can readily see that it is not a large area to examine and study, and diseased conditions in this region should not be difficult to discover, diagnose, and remedy.

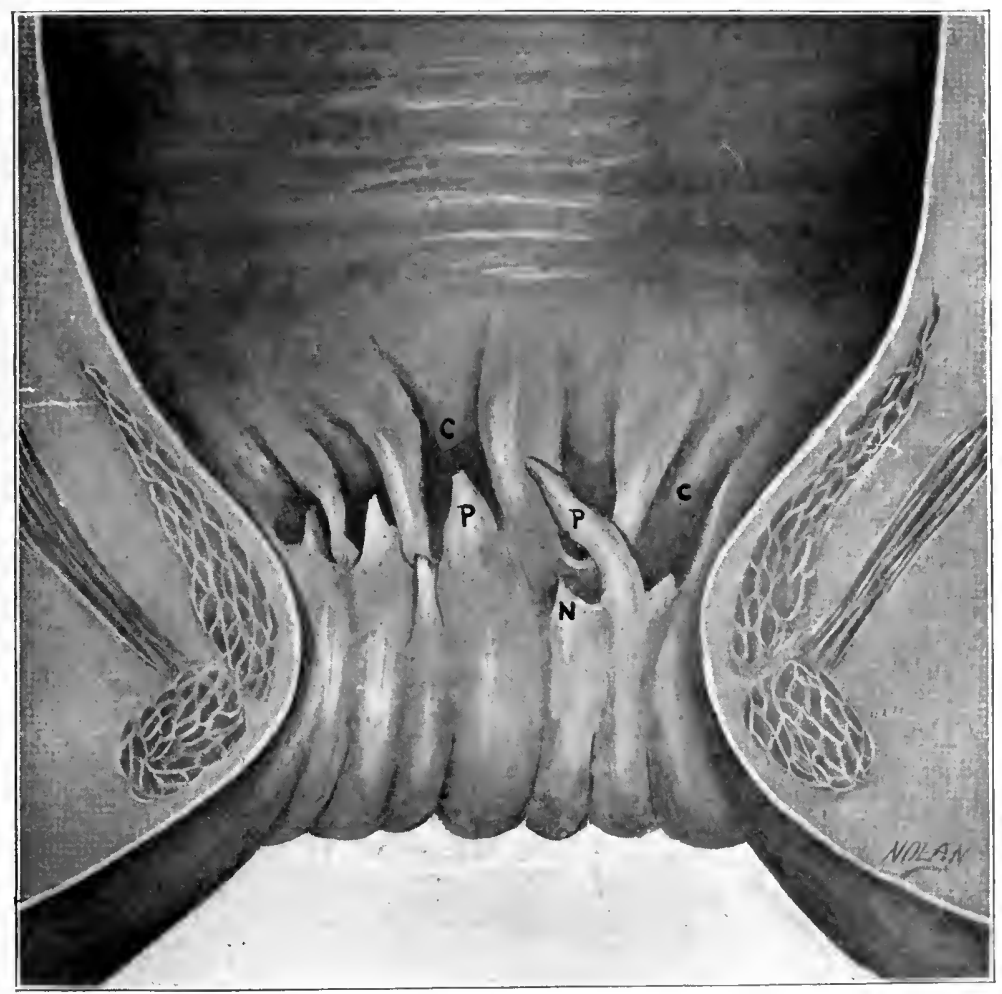

Fig. 93.

\section{Sectional View of the Anal Canal Showing Hypertrophied Anal Papil.} lae and Crypts of Morgagni.

$C$ Opening of Crypt of Morgagni.

$P$. Hypertrophied papillae.

$N$. Normal papilla. 
The anus is peculiarly susceptible to injury and disease. First, because its lining membrane being neither skin with its tough resisting power nor mucous membrane with its generous vascular supply, but a sort of transitional tissue, neither one nor the other; is easily injured. Secondly, any lesion occurring in this region has a small chance of recovery, because of its meagre blood) supply, its constantly changing position, and because of trauma and infection by the contents of the bowel which are constantly passing over it.

In order to understand more intelligently the condition under discussion it might be well to say a few words about the normal anatomy of the anal papillae (Fig. 92). These papillae occur as an irregular line of small sawtooth like projections encircling the point of the juncture of the anus with the rectum, sometimes called the linea dentata. 'These papillae, varying in number from five to a dozen, are usually situated at the edges of the semi-lunar anal valves or crypts of Morgagni. Andrews considers these papillae the normal tactile organs of the rectum and endowed with a special rectal sense. They have an abundant nerve supply, which accounts for the many reflex disturbances which originate when they are diseased.

Examination and Diagnosis.-In making a digital examination, unless one is rather expert, these papillae are not always evident to the touch, but are apt to be overlooked unless an ocular inspection is made. When diseased, these papillae may vary in size from a quarter of an inch in length, by the same breadth at the base, to an inch and a quarter or an inch and a half in the longest diameter. (Fig. 93). They are composed largely of an over-growth of normal tissue. Often by everting the 
anus, the tips, and often all of the hypertrophied papillae themselves can be brought into view (Fig. 94). They are of a pinkish color, slightly paler than the normal mucous membrane of the rectum.

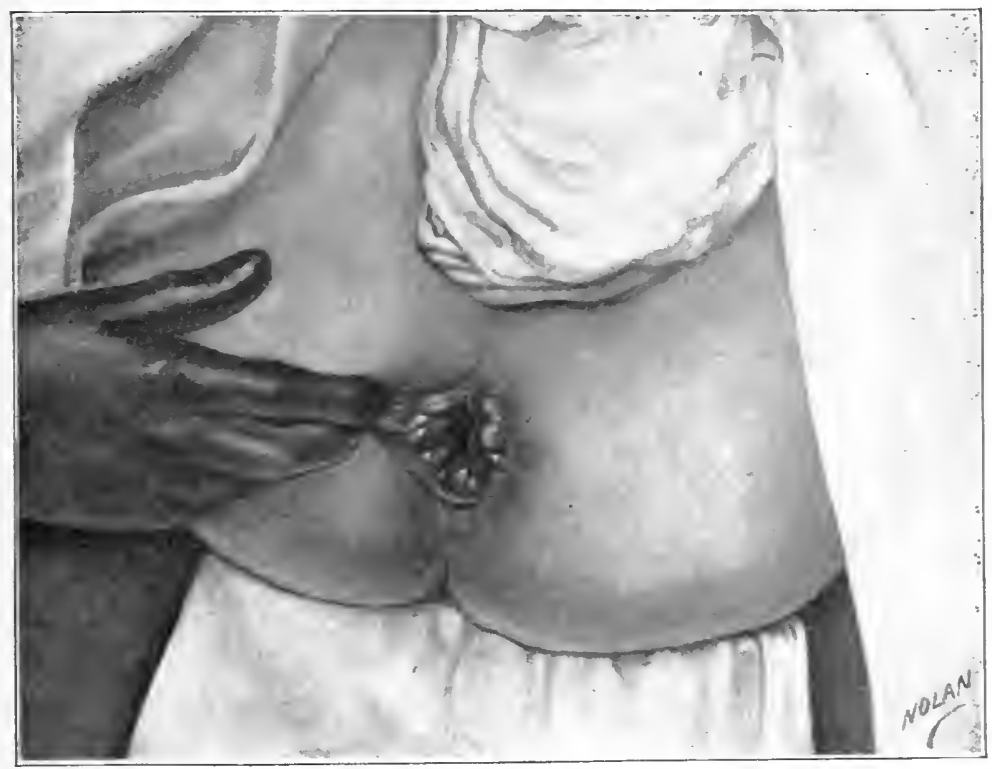

Fig. 94.

Hypertrophied Anal Papillae.

Drawn from a photograph of one of the author's cases.

This well shows the appearance of the anal papillae when the anal margin is put upon the stretch by strong traction.

A distinguishing point between hypertrophied papillae and polypi in the fact that the hypertrophied papilla is always wider at its base than the apex, while the polypus is always larger than the pedicle by which it is attached. The polypus is usually rounded or oval in shape, while the papillae is more or less triangular, or ribbon shaped. Enlarged papillae have been incorrectly designated as 
connective tissue piles. They never show the characteristic varicose appearance of the internal hemorrhoid and are attached at the ano-rectal line.

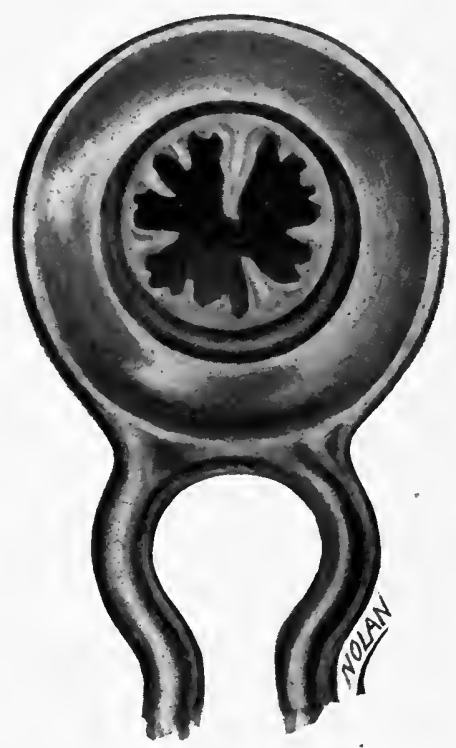

Fig. 95.

Proctoscopic View of an Exaggerated Case of Hypertrophied Anal Papillae.

Containing some erectile tissue, on examination through the anoscope, they will often be seen to stand out at right angles from the mucous membrane; giving the anal canal at this point somewhat of a fringed appearance. (Fig. 95). Many a surgeon, when he can discover no pathological lesion but finds a tight splincter, overlooks what he may call "little tags of the mucous membrane." These are very frequently the cause of the tight sphincter; for let it be said here that no sphincter is abnormally tight, unless there is some pathological 
lesion causing it; and a simple divulsion of the sphincter will not relieve the symptoms, as many a surgeon and patient have found to their chargin and disappointment.

Symptoms.-These papillae being situated on the edge of the Morgagnian crypts, are pushed and dragged downward during the passage of feces, which are more firm and harsh than normal. At each bowel movement there is a farther pull and drag on the papilla, which is gradually stretched and hypertrophied. After it has become sufficiently hypertrophied it will not retract at once after a movement, but will remain in the grasp of the internal sphincter, causing it (the sphincter) to contract. This contraction gradually becomes more tonic, and eventually we have what has been called the "tight contracted sphincter." This gives rise to one of the most characteristic symptoms of hypertrophied papillae, or that of an unsatisfied feeling after stool; a feeling as if some particle of fecal matter were still in the grasp of the sphincter and could not be expelled. Also a feeling of irritation and uneasiness, short of itching: As one patient described it to me, "It felt like the bite of some small animal," and he was sure that he had a tape worm, because he "could feel it nibbling at the anus." Another stated that it felt like a burr, held in the grasp of the sphincter. This feeling can be immediately relieved by the insertion of the lubricated finger and pushing up and replacing the enlarged papillae which will be found in the grasp of the internal sphincter. If they are left to themselves, it will often take from fifteen minutes to an hour and a half or two hours for them to gradually retract; when symptoms will entirely disappear. They cause spasm of the sphincter, and the constantly repeated 
spasm brings on a hypertrophy of the circular muscular fibres, forming the sphincter muscles, and the hypertrophied sphincter is the so-called 'tight sphincter.'"

Another symptom which the hypertrophied papillae cause is so-called neuralgia of the rectum, being transferred and transmitted pains from pressure on the nerve endings of the papillae. One of the most common symptoms, however, for which hypertrophied papillae is responsible, is pruritus ani. I do not wish to be misunderstood as saying that hypertrophied papillae are the only cause of pruritus ani, because the causes are legion; but it is a common and probably the most frequently overlooked cause.

Cryptitis. It will be remembered that each papilla is found at the edge of the semilunar valve, which semilunar valve is the outer boundary of one of the crypts of Morgagni, also known as rectal pockets or mucous crypts. These crypts, whose function is not thoroughly understood as yet, become clogged with fecal matter, which on account of the shape of the crypt or sac is not readily extruded. The enlarged anal papilla overlying the crypt assists in preventing its escape. The decomposition of this fecal matter or retained secretion and the consequent irritation of the crypt, sets up an inflammation or cryptitis. which may go on, and does frequently, to pus formation. 'The accumulated discharge originating here overflows from the crypt, and as it runs down the mucous mem. brane of the anus, sets up an irritation, which is made manifest by itching or pruritus, and the moisture complained of by many patients suffering from pruritus will be found to originate from this cause. 
The feeling of uneasiness following stool, which some patients complain of, is unlike that produced by any other condition. It has been described to me by one patient, as a feeling as if he had thorns or pine needles in the anus-a sort of prickling sensation-not painful but very uncomfortable; and he would find himself constantly shifting from side to side as he sat in a chair. Occasionally the shifting would relieve him, when assisted by some pressure on the anus, thus releasing the papillae from the grasp of the sphincter.

It is not only the extremely long papilla that we must look for to cause these symptoms; as those which are only half an inch in length, the tips of which are just engaged in the sphincter, are sufficiently long to canse symptoms.

Another condition which has been found to follow the hypertrophy of an anal papilla is fissure-in-ano. This is caused, as has been demonstrated by Wallis, of St. Mark's Hospital, London, by sufficient pressure during stool to tear the papilla downward from the edges of the crypt, and succeeding stools continuing the tearing process, the edge of the crypt is bronght down to the outside of the anus; leaving in its wake a raw, ulcerated furrow (see Fig. 61), which is split open further by each stool, and gives rise to the many severe and intolerable ssmptoms attending upon fissure in ano.

Treatment.-The treatment of this condition is extremely simple and consists in the removal of the papillae when they are enlarged, and the opening and cauterization of the crypts when inflamed or infected. Both conditions are present together so often that their treatment will be considered together as well. The removal 
of papillae is accomplished in the following manner: The anoscope, or fenestrated speculum, is inserted, with the opening directed towards the lowest papilla to be removed. The papilla is injected at its base with the onetenth of one per cent solution of eucain, or half of one per cent of chloretone, and distended to whiteness. The papilla is then removed as close to its base as possible by means of the snare, excision, or crushing. It is never necessary to anesthetize the sphincter; and oftentimes the anoscope or speculum is not required. By eversion of the anus (Fig. 94), the papilla may be brought into view and anesthetized and removed while thus exposed.

No dressing is required; the hemorrhage, which is slight, soon ceases, and no after-care is necessary, other than that employed following the operation for simple fissure.

When one of the Morgagnian crypts is inflamed, the area surrounding the crypt, including the papilla, should be injected and distended with the one-tenth of one per cent solution of eucain and a $\mathrm{V}$-shaped incision made from above; the base being at the extremities of the crypt and the apex one-half inch below its centre. This incision should be deep enough to well open the crypt. The flap, which includes the papilla, is removed, and its base canterized with chromic acid, which has been previously fused on the end of a probe. A suppository containing two grains each of chloretone, thymol iodide and powdered opium is then inserted. Where more than one crypt is involved, the same technique is employed for all; the lowermost crypt being operated first, and the others injected in turn just before operating. The after care is the same as has been described for hypertrophied papillae. 


\section{CHAPTER XII.}

\section{PROCTITIS AND SIGMOIDITIS.}

This consists of a catarrhal inflammation, either acute or chronic affecting the mucous membrane lining the rectum, sigmoid flexure or entire colon. There are many varieties of inflammation affecting the rectum and sigmoid due to the invasion by the micro-organism of gonorrhoea, syphilis, diphtheria, erysipelas and dysentery. With the exception of the last named variety, the inflammation caused by the micro-organisms of dysentery; the other varieties accompany or are caused by diseases affecting other organs and occur as a complication, and will not be described in this chapter. Amoebic dysentery will be discussed fully in a separate chapter. The author therefore will limit himself to discussion of simple catarrhal proctitis and sigmoiditis, acute and chronic.

\section{Acute Proctitis and Sigmoiditis.}

Etiology.-This disease occurs at all ages, children being affected as frequently as adults. Among the predisposing and causative factors are sudden changes in climate, weather, or mode of living; the ingestion of highly seasoned foods, condiments, and excesses in the use of alcohol or tobacco. Constipation is occasionally a causative factor, but the presence in the rectum of intestinal parasites, impacted feces or foreign bodies as well as 
infection of the rectum, from unclean enema tips or examining instruments are more often responsible for its onset. Patients suffering from rheumatism and gout or those who are peculiarly susceptible to sudden chilling of the skin surface are particularly liable to attacks of acute catarrhal proctitis. Acute indigestion, with its attendant fermentation of food products in the intestinal tract and ptomaine poisoning are very prolific sources and inflammation by extension from any acute pelvic disorder is not uncommon. The use of drastic eatharties is also an etiologic factor of no small importance, and the ingestion of many food articles which in some individnals, cause urticaria of the skin surfaces, will often be responsible for an attack of acute catarrhal proctitis.

Symptoms.-Its onset is attended oftentimes by a chill, slight rise of temperature and a sense of uneasiness in the rectum and lower abdomen; oftentimes accompanied by backache, particularly over the sacral region, and occasionally shooting pains down the limbs. This is followed in a few hours by a sense of fullness and heat in the rectum, with a constantly increasing desire for stool. Disturbances of the bladder are noted; particularly a desire to urinate frequently and a burning sensation when doing so. The patient is most comfortable lying on his side. The movements become soft and frequent evacuations occur. At first the movements are those of ordinary diarrhoea; after the first day or so, the movements consist more largely of feces mixed with mucous and sometimes tinged with blood. If the disease prog. resses and ulceration occurs, the movements contain blood and pus, and a muco-purulent discharge will be noted at the anal orifice between movements as well. In children, 
this condition frequently brings about prolapse of the rectum, and oceasionally also in adults.

Diagnosis.-With the history of an onset such as has been given above, examination of the rectal cavity is indicated. With the patient in the knee shoulder position the proctoscope should be inserted and the rectum inflated. If the insertion of the proctoscope is accompanied by considerable pain, as it will be in many cases suffering from proctitis, the sphincters should be first anesthetized according to the technique outlined in Chapter XV. The appearance of the mucons membrane of rectum is somewhat characteristic. Upon ocular examination, the rectal mucons membrane is bright red in color, its appearance being not unlike the appearance of the inflamed conjunctiva, the difference being that the rectal mucous membrane will be more of a brick red, and the mucous membrane appears somewhat velvety and oedematous. An increased quantity of stringy, yellowish colored mucous will be noted. The blood vessels of the rectal wall, and particularly on the valves of Houston, will be found deeply injected and elearly ontlined, standing out distinctly from the red mucons membrane.

Treatment.-The treatment of acute catarihal proctitis is dietary, systemic and local. In those cases depending for their origin upon the presence in the rectum or sigmoid of impacted feces or foreign bodies, their removal is first indicated. Where the proctitis is caused by ptomaine poisoning from decomposition of food material in the intestinal tract, prompt and free catharsis is the first essential. Patients suffering from systemic or constitutional diseases in whom the proctitis is merely a complication, should of course receive general medical 
treatment for the underlying constitutional or systemic trouble.

Where irritating or improper food material is the causative factor, or the indulgence in alcoholic stimulants or tobacco to excess is responsible, their interdiction and withdrawal is obvious.

In the local treatment of acute catarrhal proctitis, copious irrigations of the rectum sigmoid and colon with normal saline solution, at a temperature of 110 to 115 $\mathrm{F}$, given twice or three times during the twenty-four hours, has in many cases been sufficient.

In irrigating the colon, the position in which the best results are achieved are either the knee-shoulder, left lateral or Sim's, or the lithotomy. Where either of the latter positions are employed, the hip should be elevated considerably higher than the head. The irrigator, or fountain syringe, to be placed from one and a half to two feet above the level of the anus, and the flow checked by pressure on the tubing, when there is a desire on the part of the patient to expel the fluid before a sufficient quantity has been administered. This uncomfortable feeling is due to the over-distension of the bowel at certain points when the inflow is interrupted either by the normal sacculations or spasmodic contraction of the circular muscular fibres. This sensation will soon pass away, however, if the inflow is checked for a moment so as to allow the solution already in the bowel to flow higher up. Changing the position of the patient from one side to the other and massaging the abdomen gently will greatly assist in the distribution of the irrigating fluid. By this method, the majority of patients will be able to re- 
tain a sufficiently large amount of the irrigating fluid to thoroughly flush the entire colon.

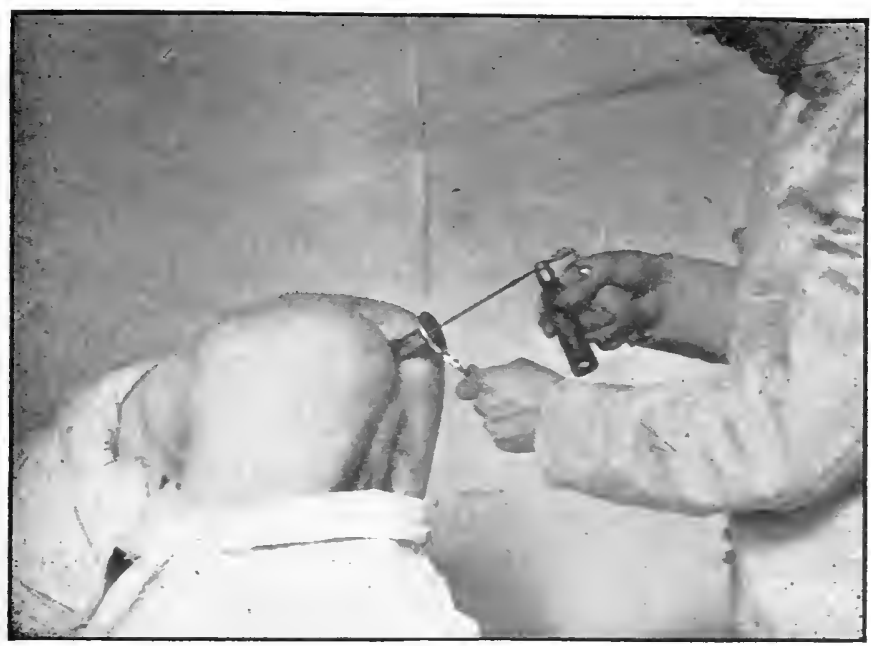

Fig. 96.

Spraying the Rectum With the Patient in the Knee-Shoulder Position.

On account of the ballooning out of the rectal cavity by air inflation in the knee-shoulder position, this position is ideal for the application of sprays to the rectal surfaces.

In those cases where the mucous discharge from the rectum or sigmoid is profuse, the use of nitrate of silver solution in strengths ranging from one to five per cent, by means of the rectal spray, has been found very efficacious. The author uses a metal spray tube attached to the hand atomizer or used with the compressed air tank, which is nine inches in length. Its distal extremity is closed, but from its circumference, about one-sixteenth of an inch from the end, the solution issued in all directions from four small apertures, so that the solution is not thrown any higher into the bowel than one wishes, 
but bathes all surfaces alike (see Fig. 97). The rectum and sigmoid is best sprayed with the patient in the kneeshoulder position (Fig. 96). In some cases, where the mucons flow appears to come from higher up in the bowel, irrigations of the colon with various astringent solutions, are indicated. Two to five per cent solutions of alum answer very nicely, and the aqueous fluid extract of Krameria from five per cent to twenty per cent as advocated by 'Tuttle, has proved of value in the author's hands.

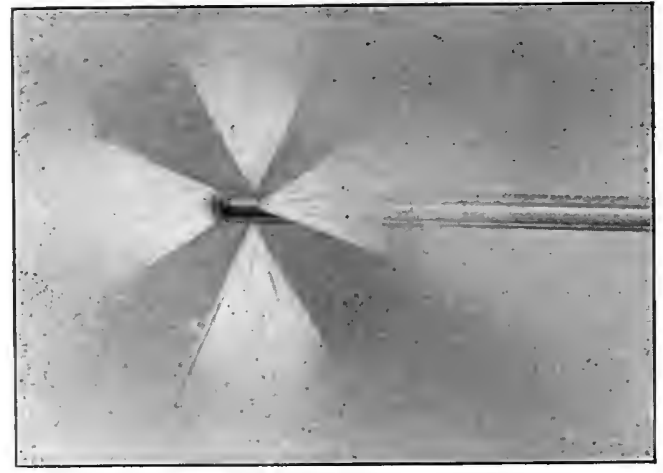

Fig. 97.

Author's Rectal Spray Tube.

The tip being closed and the solution issuing from its circumference only, it is impossible to throw irritating solutions like nitrate of silver higher into the bowe! than one wishes.

While many authors advocate the confining of the patient to bed during the treatment for acute catarrhal proctitis, the author has found no difficulty in securing results by allowing the patient to be up and around for a greater portion of the day. He believes that better drainage of the intestinal tract is secured at all times by the upright position. In some cases where results are not obtained by spraying with aqueous solutions, and where 
there is a tendency for the bowel walls to ulcerate the insufflation of various powders will be found of great value-iodosyl, compound stearate of zine with balsam peru or boric acid, thymol iodide, have all been found very satisfactory in these cases.

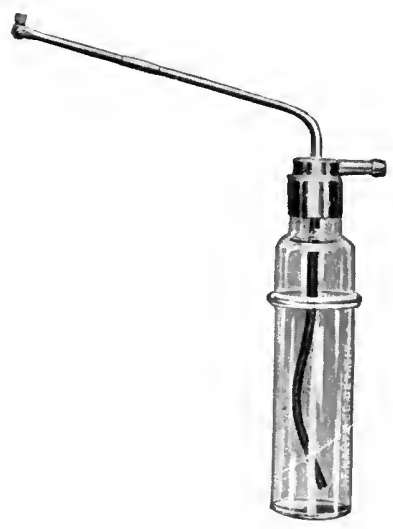

Fig. 98 .

DeVilbiss Spray Tube.

Provided with an adjustable tip so that the spray may be thrown in any direction.

Ulcerating spots should be touched up with pure ichthyol or solutions of 5 per cent or 10 per cent of nitrate of silver. The author is not in sympathy with the use of solutions of the stronger chemical antiseptics such as the bichloride of mercury or carbolic acid, even when used in very weak solutions; he believes that more harm is accomplished by the action of the irritating chemical solutions on the weak and debilitated lining mucous membrane, than whatever little good they accomplish by their action as antiseptics.

In irrigating or flushing the colon, the recurrent flow soft rubber colon tube devised by J. L. Jelks, of M[em- 
phis, Teun., will be found a very useful piece of apparatus. For the technique of its use the reader is referred to the following chapter on dysentery. During the treatment of a case of proctitis or sigmoiditis, the patient should be kept on a light and unirritating diet in which the vegetable elements are largely eliminated. The thin cereal gruels prepared from oatmeal, rice and barley, egg albumen, the various flavored gelatines and liquid peptone solutions will be found best for use in these cases. Milk is contraindicated on account of its tendency to constipate, and the fact that it forms hard curds which only further irritate the already sensitive bowel.

Internal medication is not of much avail; the use of ichthyol in 2 to $5 \mathrm{gr}$. doses given in double capsules four times daily, the author believes has given some good results. He has found the employment of white refined petroleum oil or liquid albolene to be of particular value in proctitis. It seems to have a specially soothing effect on the inflamed and irritated mucous membrane of the bowel and while it does not produce or stimulate peristalsis, it causes easy and free evacuation by its mechanical softening and lubricating effect. Being a mineral oil of no food value and having no medicinal effect, it is not acted upon by the digestive secretions, and passes through the intestinal canal unchanged.

The patient should be instructed to drink six to eight glasses of water daily; if there is any doubt as to the purity of the water, it should be boiled and then kept in bottles on ice. In order to remove the flat taste of boiled water, the author would suggest that before use it be poured into an open vessel or pitcher and stirred up with a revolving egg beater. This aereates the water so that 
it again tastes fresh and clean, and effectually removes the unpalatable taste which is one of the drawbacks to the use of water sterilized by boiling. The use of flaxseed tea is often of assistance in these cases. If properly prepared is of distinct value. A good way to prepare flax-seed tea is as follows: Take four or five tablespoonsful of whole flax seed and place in a shallow pan. Pour over this a quart of boiling water, place the pan over the flame and allow to boil for five minutes, then strain through muslin and allow it to cool. It is best kept on ice until ready to use. If it is desired to sweeten or flavor the flax-seed tea, lemon juice, oil of peppermint or wintergreen and sugar may be added in quantities to taste while the tea is still hot. A teacupful should be taken as hot as can be comfortably borne every night at bed time. This will act, often, as a mild cathartic and seems to have some soothing influence on the mucous membrane of the bowel.

\section{Chronic Proctitis and Sigmoiditis.}

This disease is usually of two varieties, hypertrophic and atrophic. The atrophic variety is the most common variety of chronic proctitis or sigmoiditis met with. The hypertrophic variety may follow an attack of acute proctitis or sigmoiditis but is often produced by other diseased conditions outside of the bowel. Pressure from abdominal tumors, movable kidneys, uterine displacements, extension from pelvic cellulitis and adhesions following inflammatory conditions of the pelvis may all set up attacks of hypertrophic proctitis and sigmoiditis. Appendicitis has also been mentioned as an etiological cause.

The atrophic variety may often be brought about by a long period of chronic constipation; the abuse or ex- 
cessive use of cathartics extending over a long period of time; excesses in both eating and drinking, particularly in people of sedentary habits. Other causes of a more local nature are repeated attacks of fecal impaction; the enema habit, foreign bodies in the rectum and unnatural practices.

Chronic Hypertrophic Proctitis. This variety is distinguished from the atrophic variety by the fact that the mucous membrane and submucosa are always thickened, and the glands as well as the inter-glandular connective tissue hypertrophied and increased. The anal papillae are usually very much enlarged in this condition. On proctoscopic examination, the appearance of the mucous membrane is somewhat characteristic. Tuttle well describes it as follows: "Through the proctoscope it appears oedematous, paler than usual and covered with a thin coat of whitish secretion. The swollen membrane bulges out into the fenestra of the conical speculum, or falls down and completely covers the end of the proctoscope. When the muco-pus is wiped off, the membrane presents through the magnifying glass a cauliflower-like appearance, whitish and granular. It does not bleed easily, and the end of a fine probe being pressed down upon its surface, the tissues will meet together above it. By scraping with a rectal scoop one may obtain a certain amount of muco-purulent fluid, consisting of pus cells, leucocytes and various bacteria, together with small masses of fecal matter and undigested particles of food."

Symptoms.-The disease may be of insidious onset, or it may be the continuation of an attack of acute catarrhal proctitis. The patient is usually in a run-down 
condition, and presents the usual symptoms of such a state, such as impaired appetite, foul breatl, indigestion, gaseous eructations, diarrhoea, occasionally alternating with constipation, a frequent desire to move the bowels without much result and an unsatisfied feeling as if something more were to pass away after a stool. Where the bowels are loose, the stools are inclined to be of a pea soup consistency, consisting quite largely of muco-purulent material, or there may be small hardened boluses or scybala covered with sticky mucus, or muco-pus. On account of the hypertrophied condition of the mucous membrane, prolapsus is met with in some cases, and pruritus ani is a frequent symptom. The secretion keeps the region of the sphincter constantly moist and is occasionally so profuse and constant that the patient has to wear an absorbent dressing to prevent it from soiling the clothes. On account of the constant moisture of the part, condylomata are occasionally found, particularly at the posterior aspect of the anus and anal canal.

Diagnosis.--The diagnosis is made upon proctoscopic and sigmoidoscopic examination. The characteristic hypertrophied appearance of the nucous membrane, with the presence of muco-purulent discharge, with or without ulceration of the mucous membrane, accompanied by a history of symptoms such as have been given above, should make the diagnosis not difficult. The condition is, fortunately, not very common.

Treatment.-If upon examination of the patient such extra-rectal causes as appendicitis, floating kidney or abdominal or pelvic growths impinging upon the bowel are discovered, the indicated surgical measures for their relief should be carried out. 'The patient's dietary 
should be corrected and all condiments, alcoholic stimulants, pastries, salads, sweets, fresh fruits and freshly baked foods prohibited.

In order to give as little work to the intestines as possible, the patient should be put on a diet which is largely assimilable, such as: eggs, the various gelatins, lean meat, poultry, fresh water fish and small quantities of green vegetables such as spinach, beet tops, lettuce, endive and kale. The patient should be encouraged to drink large quantities of cold water and should try to have a bowel movement at regular hours. Liquid albolene in doses varying from one to four drachms three or four times a day should be administered on account of its soothing influence upon the mucous membrane of the intestinal tract, and because by its admixture with the feces, it prevents the formation of hard, irritating masses.

Where symptoms of intestinal indigestion are present the author has found pancreatin in ten-grain doses, taken with or directly following the meal, of considerable value. Ichthyol in double capsules, in doses of from two to five grains four times daily seems to be of some service. The bowels should be flushed morning and night with some astringent solution such as is used for the treatment of acute catarrhal proctitis. Tuttle recommends very highly the use of one to three quarts of a two to ten per cent solution of the aqueous fluid extract of krameria. This is best given with the patient in the kneeshoulder position and through a Jelks' recurrent flow colon tube. The preparation of the aqueous fluid extract of krameria as described by Tuttle is as follows:

"Macerate one pound of bark of krameria in a long percolating tube for twenty-four hours. After this a 
mixture of 20 per cent glycerin and 80 per cent water is allowed to percolate through it. The percolate should be constantly stirred and refiltered through the bark the second time. The filtrate is then evaporated down to one pound, thus obtaining an aqueous fluid extract con-

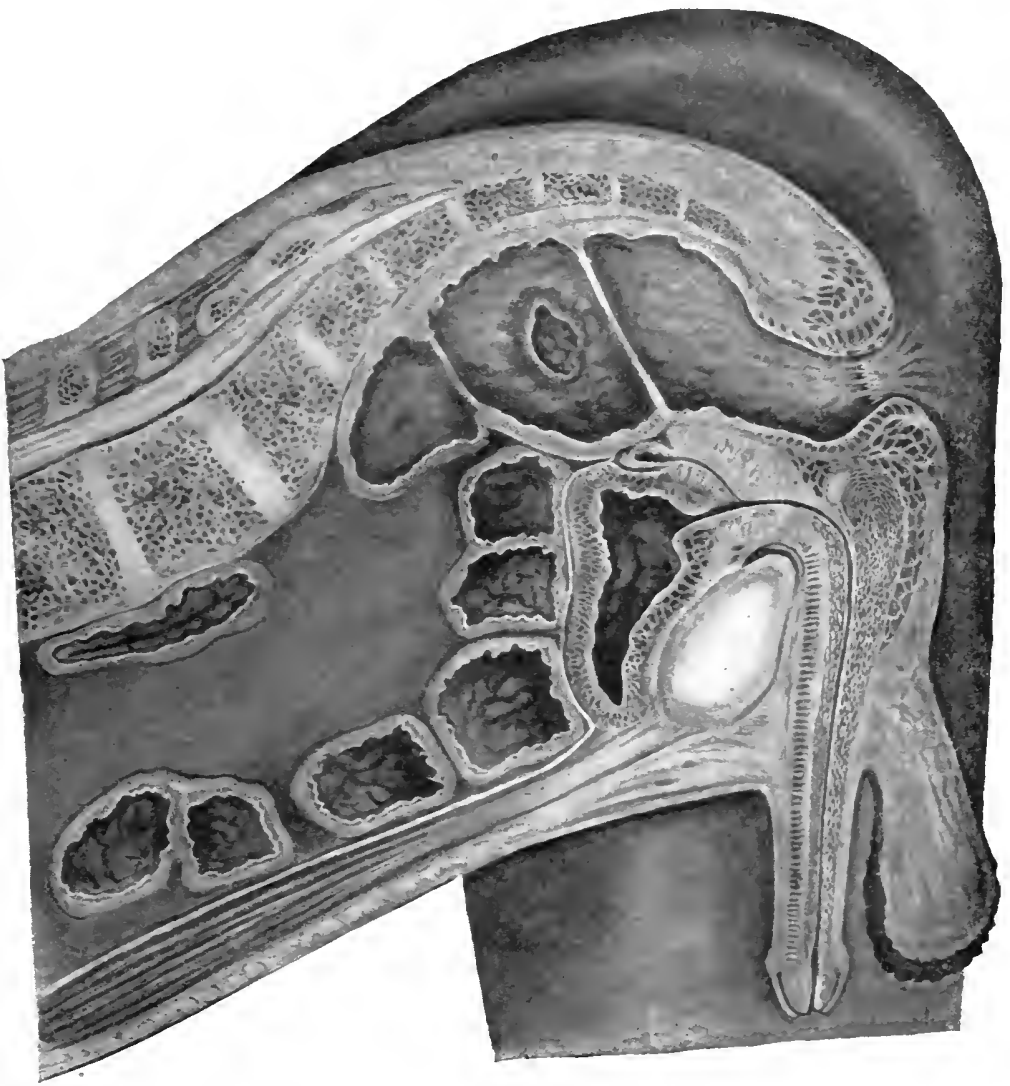

Fig. 99.

Ulcer of the Rectum.

This case well illustrates the importance in proctoscopy of examining thie cavity behind each rectal valve. In this patient the ulceration was situated on the right lateral wall of the rectum, and had not the first riectal valve been pushed aside by the proctoscope its presence might have escaped unnoticed. 
taining grain for grain all the therapeutic properties of the bark. The preparation should be kept in a dark place and not exposed to the air.',

If, on proctoscopic or sigmoidoseopic examination localized ulcerated areas (Fig. 99) are discovered, they should be sprayed with a 1 to 3 per cent solution of nitrate of silver or 5 per cent solution of ichthyol. They may be stimulated by the application of nitrate of silver 5 to 10 per cent applied with a long handled applicator or pure ichthyol or balsam peru. The general condition of the patient must be improved by ordinary tonic measures and the encouragement of moderate exercise in the open air and sumshine.

\section{Chronic Atrophic Proctitis and Sigmoiditis.}

This variety is more common than the hypertrophic, and consists of a general atrophy of both the glands and intra-glandular structures of the rectum and sigmoid. It differs from the hypertrophic variety in that it does not frequently extend higher than the sigmoid flexure and there is a thinning or destruction of the mucous membrane lining of the bowel. The pathology of the condition is well described by Tuttle as follows:

"One observes upon examining the mucous membrane in these cases an irregular, bosselated or granular appearance. The surface is dry, rough, inelastic and without any salient vegetations. Attached to the surface here and there are small masses of dry fecal material, and occasionally little islands of necrotic epithelium or pseudo-membrane.

"Microscopic examination shows the epithelium absent in many places, but always present in the deeper por- 
tions of the crypts of Lieberkühn. These follicles are generally atrophied, the intratubular tissue decreased, and their goblet cells are few in number. The cylindrical epithelium is said to assume the stratified pavement type in this disease. This change does not extend more than 1 or 2 centimetres above the ano-rectal line; it is confined to the superficial structure of the membrane, and does not involve the tubules.

"The connective tissue of the submucous coat is dense and slightly thickened; it does not contain embryonic tissue and elastic fibres, as in the hypertrophic form. The solitary follicles are often enlarged and distended. At points there are distinct granulations, and ulcerations, accompanied with hyperemia and multiplication of the blood vessels, but there is no alteration in the blood vessel walls."

Symptoms. As has been stated, this condition supervenes frequently on an old long standing case of constipation. The stools are small, hard and dry, and their passage is painful; they are often streaked with blood. pus and mucous. The patient suffers from tenesmus, referred pain in the sacral region and down the legs. The rectum feels hot and feels after stool, as if it were not emptied. This feeling is not like the sense of fullness which is more characteristic of the hypertrophic variety; but more a sense of uneasiness which focuses the patient's attention upon the rectum, which makes him feel that the emptying of the rectum will bring him relief. Pruritus ani is a frequent stmptom as well as spasm of the sphincters. On account of the contracted condition of the anal canal, the passages are frequently followed by the production of small fissures or cracks 
in the mucous membrane. Their presence adds a stinging or burning sensation to the other symptoms of the disease. These fissures are very superficial and are not to be confounded in any sense with the true or typical fissure in ano. They consist merely of linear abrasions in the lining membrane of the anal canal, and lack any tendency to chronicity which is characteristic of a true fissure. Hemorrhoids are said to be found frequently accompanying this condition.

With the patient in the knee-shoulder position, proctoscopic examination shows the mucous membrane to be reddened but not markedly as in the acute variety; dry, and covered here and there with small flecks of dry fecal matter. The insertion of the proctoscope is usually accompanied by some hemorrhage due to the passage of the instrument. On examination of the rectal walls numerous pin point ulcerations are met with. The mucous secretion which is very slight in this condition, clings to the bowel wall, and is characterized by thickness and tenacity. In this condition the mucous membrane does not fall together before the proctoscope and the rectum gives the appearance of being a stiff tube, while the rectal valves stand out very markedly. Ulcers other than the pin point variety are not uncommon, and tend to become chronic and to gradually encircle the bowel, producing a strictured condition.

Treatment.-In this condition the presence or absence of syphilis should be ascertained. Where either from the ignorance of the patient of his true condition or from his reticence about the matter one cannot obtain a definite history, the Wasserman test, or serum diagnosis should be resorted to. If positive, the ordinary 
measures for the treatment of syphilis in the third stage should be employed. The diet is exactly the same as that outlined for hypertrophic proctitis, with the exception that the patient may have fatty food, bread (not freshly baked), toast, rice, sago and custards. Where intestinal indigestion is present pancreatin should be administered and liquid albolene given as outlined in the treatment of the hypertrophic variety. As this condition is usually confined to the rectum and lower sigmoid, the high irrigations will not be necessary, but the solutions mentioned are equally applicable for the flushing of the sigmoid and rectum in this variety. After irrigating the rectum, the patient should be put in the kneeshoulder position and under the guidance of the eye, ulcerated patches on the mucous membrane should be touched up through the proctoscope with 2 to 5 per cent solution of nitrate of silver, iodine or pure icthyol. Icthyol in 5 per cent aqueous solution is very valuable as a spray in this condition, as is the fluid extract of krameria in strength ranging from 20 to 30 per cent. The treatment of the accompanying conditions such as fissures, hemorrhoids and pruritus should be carried out as outlined under the respective chapters. What has been said before regarding exercise and fresh air is equally applicable in this condition. 


\section{CHAPTER XIII.}

\section{DYSENTERY.}

By Johr L. Jelks, M. D., Memphis, Temm.

Synonyms.-Colitis, Ruhr or Dysenterie (German),

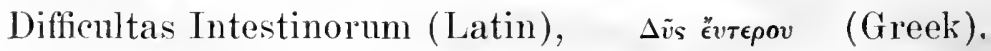

Definition.-An acute or chronic inflammatory disease, usually affecting the large intestine, beginning in the rectum but sometimes extending into the small bowel. In the acute form it is characterized by pain, tenesmus, and frequent passages of bloody mucus. In the chronic form the patient suffers recurring attacks of diarrhoea alternating with constipation.

Historical.-Dysentery was one of the best known diseases of antiquity. Even before the time of Hippocrates, reference to it was made; the earliest being that formd in the papyros of Ebers'. Hippocrates, in the year 460 B. C., was the first writer to give a fairly accurate description of its symptematology, pathology, and sequellae.

Other well known writers were Celsus, the medical Cicero of his day (45 A. D.), Aretaeus, Galen, and Alexander of Tralles. Then for more than a century little further knowledge was imparted until the time of Antonio Benivieni (1506), and Thomas (1833), who refuted many of the erroneous ideas of his predecessors. Woodward 
(1879) gave a most excellent history of this disease (Medical and Surgical History of the War of the Rebellion, Vol. 2). Kartulis, in Egypt (1885), Flexner (1890), Councilman and Lefleur (189:), Shiga of Japan (1897), Strong and Musgrave, and Harris, of Atlanta, of the present era, have contributed perhaps the most valued writings. Osler, Tuttle, and Surgeon General Sternberg of the United States Army are also among those who have furnished valuable data in our own country.

The writer of this chapter has also made close study of this disease in the Southern States.

Geographical Distribution.-Dysentery does not respect any country, climate or race. Ayers very truthfully states, that, where man is found, there some of its forms appear. A. Hirsch says it had a wide distribution over the inhabited earth at all historic times. It is without doubt one of the four great epidemic diseases of the world. In the tropies its ravages have been most deadly, destroying more lives than cholera, and to the armies it has been more destructive than powder and shot (Osler). Dysentery is a destructive giant compared to which strong drink is a mere phantom (MeGregor). The worst outbreaks occur as endemics in the tropics and decrease as we leave this latitude, while in the higher latitudes it seldom appears in this type, though now and then in greater or less epidemies. A very striking fact relative to this affection is that it involves the cold zones. Epidemies have been reported in Alaska, Sweden. Russia, Greenland, and Iceland, also others of the colder countries.

General Etiology.-Season.-Among the predisposing causes season is the most important. More cases of 
dysentery are found during the summer and autumn months. This is due to several reasons. Sudden changes in temperature, especially sudden rises, have a most marked effect. It is most prevalent in the warm climates, and, as stated above, it is most deadly in the tropics. Therefore, climate should be mentioned as one of the causative factors.

Race.-Race itself does not seem to affect this disease. Strange, though it may seem, the negro race in the South has not seemed to suffer much, with reference to this disease; notwitlistanding the baneful consequences of poor hygienic conditions as overcrowding, improper food, poor ventilation, filth, thin clothing, and especially syphilis-a disease almost universal among this race, either inherited or acquired. These, however, must all be included under predisposing causes.

Sex.-Under etiology, we should also mention sex. Within our experience, which is not at variance with that of other writers, dysentery is much more common among males.

Poor Hygiene.--In the slums of our cities, where filth abounds and where proper sewerage is lacking, we find more cases of dysentery than in the sections where the hygienic conditions are better. Many cases are found in institutions such as insane asylums, barracks, jails and army camps. Wherever there is over-crowding, there is very apt to be found a large percentage of dysenteric cases. During the Civil War, Woodward reported 259,071 cases of acute dysentery, and 28,451 of the chronic form, in the Federal service alone.

Topography and Conditions of Soil.-Investigators have tried to associate dysentery with certain topographi- 
cal conditions, or with conditions in the soil, but have been unable to do so.

Epidemies have proven more fatal in the country than in the city.

Soil that is badly contaminated with dysenteric excreta is a great source of infection. Czernicki tells about dysentery breaking out in two French squadrons in 1875 that were on the same ground occupied a short time previously by a cavalry regiment which had been affected with the same disease.

The writer has often found nests of dysenteric cases in the low flat mill districts of the city, and in marshy lowland sections of the country. No doubt, owing to the character of the soil in these localities, seepage contamination of drinking water sources sometimes occurs. Houses built upon a low damp soil are unsanitary, and, when the surrounding soil always remains saturated with moisture, there exists a favorable condition for the development of dysentery. The peculiar emanations from soil of this kind have always been considered very harmful. It is thought that they have a depressing influence upon the inhabitants, and thus make easier the inroads of diseases.

In one ill-drained low district of Memphis, the writer treated six cases of amebic dysentery within a radius of two blocks, and a seventh ease was seen that had been infected in the same territory five years before. It is also an interesting fact to note here that two families, in which were four of the patients, purchased vegetables from the same Italian huckster.

There is yet another reason why we find more cases in the marshy low-land districts. Here we find the greatest 
growth of vegetation, which, when conditions are favorable, furnish a most suitable nidus for the propagation and development of amebae, bacteria, and other microolganisms.

Foods.-Certain articles of food are unquestionably predisposing causes in dysentery. This fact is not due so much to an idiocyncrasy to any particular foods, but mainly to the micro-organisms which they contain, and to the putrefactive changes which occur within the intestinal tract. All grounding vegetables and fruits, especially those shipped from the tropics, are possible sources of infection.

Undoubtedly infections with the amebae have been traceable to eating such vegetables as lettuce, strawberries, cress and potatoes.

Eating food in excess, and the resulting attacks of indigestion often pave the way for dysentery.

Drinking Water.-The writer has given much thought to water supply as a medinm through which dysenteric infections are conveyed. This is undoubtedly the most common source. We have been impressed by the fact that many cases are found among sportsmen, also timbermen who spend much of their time in the woods, and who drink, when necessity requires, from surface pools; springs and slashes.

The writer has treated a case of amebic dysentery from a cointry district with which he is quite familiar, and has knowledge of the fact that the disease was contracted in the same infected neighborhood in which twenty years previous another case had lived, which proved fatal. The fact has been elicited that many of the rriter's cases had neighbors suffering in like manner, 
and who were procuring their drinking water from the same source.

There are certain rivers in China whose waters are known to cause dysentery.

In 1863, the number of cases among the workmen constructing the Snez Canal was decreased when the better water of the Nile was used.

The writer has treated one case of amebic dysentery in the person of a physician, who thinks undoubtedly that the infection was obtained from drinking Mississippi River water while on board a river steamer. Thevenol says, "Nothing is so prone to lead to disorganization of the large intestine as infected water." Impure water itself does not produce dysentery, but only when it contains the special micro-organisms.

\section{Classification.-Acute Catarrhal Dysentery or Spor- adic Bacillary Dysentery:}

This form is the least severe and most common form that is encountered. It occurs both sporadically and endemically. This type is characterized by the frequent passage of great quantities of mucus.

Special Etiology.-Children principally are infected with this form, but we often see it in adults, most often complicating other diseases. It is the kind of dysentery that accompanies all of the exanthemata. We see it in fact, complicating almost all the acute infectious diseases. Still another important cause is the ingestion of certain kinds of foods, or other irritating substances. The ordinary attacks of entero-colitis in babies during the summer months come in this elassification. Most of these attacks are due to milk poisoning. 
Pathology.-Macroscopically a superficial, acute inflammation involving the large intestine, but sometimes extending into the small bowel, is seen. The tendency of such cases is to recover without necrosis. Sometimes, though, in the more severe attacks, the mucosa will become injected to such a degree that small ulcerations occur. In these cases the mucus is often stained or streaked with blood.

Microscopically, are seen the bacillus coli communis, also the trichomonas intestinalis, and paramoecium coli, and occasionally the cercomonas intestinalis. We also find red blood corpuscles and leucocytes, and always large numbers of desquamated epithelioid cells, dotted about with fat globules and vacuoles.

Symptoms.-The onset is sudden and usually ushered in by an attack of cholera morbus, or by an attack of acute indigestion. Sometimes a more or less distinct chill may occur at the onset.

Nausea and vomiting are not rare symptoms.

The tongue has a moist coat at first but soon becomes dry.

From the first there is diarrhoea. Pain is complained of over the entire abdomen, also tenesmus, and severe griping pains. 'The patient is extremely restless and can not get relief from a desire to stool. The bowel movements are at first free, and watery, or sero-sanguinous, but later on, contain only small quantities of mucus streaked or stained with blood, and have an offensive odor.

A slight elevation of temperature usually accompanies this form, but, in more severe cases, it may reach 103 
degrees F. There is corresponding acceleration of the pulse, and the patient complains every few minutes of thirst.

The stools, during the first day or two of the attack, contain, in addition to the above mentioned materials, small fecal masses (scybala). Sometimes during the course of the attack, the stools contain an excess of bile and cause intense burning while passing.

The ordinary cases of acute catarrhal dysentery are self-limited, usually recovering in a week. Some are so mild that treatment is not sought. It must be remembered, however, that the cases which begin with mild symptoms may develop graver ones at any moment.

Diagnosis.-The diagnosis is very easy. The cramping pains, tenesmus, and frequent passages of mucus and blood are positively diagnostic. If, however, a case may be obscure, the microscope and proctoscope will at once clear it up.

Prognosis.-In most cases the prognosis is favorable, but it is best to be guarded at all times in giving it, since some of the cases, which, at first seem quite mild, may terminate adversely. Ordinarily, though, the symptoms will subside in a week, and the patient will recover rapidly. There is always rapid emaciation and weakness.

\section{Diphtheritic Dysentery.}

Definition.- This is an inflammation, usually confined to the lower part of the colon, and rectum, but sometimes extending into the small bowel. It is accompanied by a croupous, or true diphtheritic exudation. It is one of the epidemic forms found in Japan, also in armies, in insane 
asylums, and ships, or wherever large numbers of people are crowded together.

Etiology.-This form of dysentery is caused by the Bacillus Dysenteriae, discovered by Shiga in Japan (1897). Flexner and Strong encountered the same bacillus in one of the forms of the disease which prevails in the Philippines and Porto Rico. The bacillus is described by Shiga as being a short rod with rounded ends, and closely resembling the bacillus of typhoid fever. It possesses slight motility. Flexner discovered that the bacillus "Is inactive to blood serum from tỵphoid fever cases, but reacts with serum from dysenteric cases to which bacillus typhosus does not respond." Shiga's bacillus may be found within the body as late as one year after the primary infection.

Pathology.-The mucosa, if the attack is not severe, is coated over with a yellow exudate. Slight ulceration of the mucous membrane over the tops of the folds of the colon are seen.

In severe attacks, however, all the layers of the colon are involved, and it appear's greatly enlarged. The infiltration is so great that extensive necrosis takes place. The mucous membrane over the entire colon presents a puffy or swollen condition, yellow in color. Large areas may slough en masse.

Microscopically, this slough is found to consist of a fibrinous and cellular exudate coating over the mucosa.

The glands of Lieberkühn are destroyed, and sometimes no trace of them is found.

Symptoms.-The symptoms are practically the same as those of acute dysentery greatly intensified. The onset is more severe. The chill is often present, and the 
fever is high, rumning an irregular remittent course. The pulse is greatly accelerated, tormina and tenesmus are most severe.

Delirium is common. Bowel movements may at first be loose and watery. Soon great quantities of sero-sanguineous discharges, containing bloody muco-purulent material and slonghs of variable sizes are passed. The distension of the abdomen is greater and pain is more severe. There is more rapid loss of strength.

Diagnosis.-The diagnostic points of most value are the character of the dejections, which may contain pseudo-membranes, severe simptoms, and the appearance of epidemics.

The positive diagnosis is by the agglutination test.

Complications.-Complications in this form are encountered inore frequently.

Perforations sometimes occur, and are almost invariably followed by peritonitis.

Liver abscess is another grave complication.

Nephritis, phlebitis, pericarditis, endocarditis, and pleurisy have also been noted.

Recovery sometimes takes place, but usually after a more or less chronic course.

\section{Secondary Diphtheritic Dysentery.}

The lesions of this form are similar to those of the last described, but not so severe. The secondary, as the name implies, usually follows one of the acute, or chronic diseases, as pnemonia, nephritis, pericarditis, endocarditis, pulinonary phthisis, typhoid fever, and numbers of other varieties. 
Symptoms.-The symptoms are sometimes not very noticeable. The griping pains and tenesmus are not very severe as a rule. The patient has about two to six loose bowel movements a day. Anatomically, the inflammation is very superficial, only the upper layers of the mucosa being involved. The inflammation may progress producing more or less necrosis. Very little blood is found in the stools.

Prognosis.-The patient will often perish. Owing to adynamia already existing, much resistance is impossible.

\section{Amebic Dysentery.}

Synonyms.-Amebic colitis, amebic enteritis, amebiasis.

Dysentery in this form is both epidemic and endemic in the tropical countries, especially India, Africa, and the Philippine Islands. In the United States sporadic cases are met with frequently. Osler says, his cases in the Johns Hopkins Hospital were almost exclusively amebic. It is very rare, indeed, that the writer is called upon to treat a case of the severe acute or chronic type in which he is unable to make a positive diagnosis of amebic infection by means of the microscope.

This is the prevalent type of the grave chronic and relapsing cases of dysentery in this country, and many of the supposed diphtheritic dysenteries are of this origin. The microscope alone, however, can verify or refute this opinion.

Etiology.-This form of dysentery is caused by the entamoeba histolytica or the amoeba dysenteriae (Fig. 100). (Councilman and Lefluer.) 
It is a type of protozöön, unicellular, and motile, several times the size of a red blood corpuscle. In structure

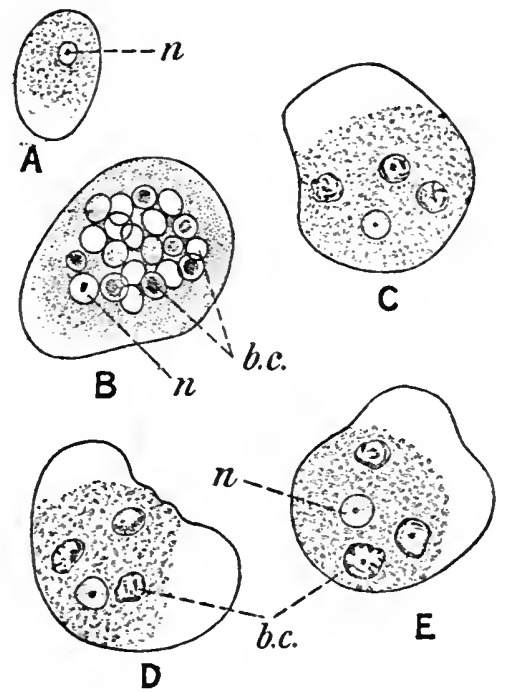

Fig. 100 .

Amoeba Histolytica Schaud.

A, young specimen; $B$, an older specimen crammed with ingested bloodcorpuscles; C, D, E, three figures of a living amoeba, which contains a nucleus and three blood corpuscles, to show the change of form and the ectoplasmic pseudopodia; $n$, nucleus; b, c, blood corpuscles.

After Jurgens, from Allbutt's System of Medicine.

the organisms have an outer colorless zone called the ectosarc or hyaloplasm, and an inner granular zone, the endosare or endoplasm. Its nucleus is eccentrically situated, and one or more vacuoles is present. This parasite is phagocytic in character, and may be seen to contain red blood cells, vacuoles, and other particles. It is easily mistaken for a large epithelial cell, or paramoecium, when not in motion. It is ten to fifty microns in size. The amoeba hystolytica multiples by segmentation, the nucleus and endoplasm dividing in such manner as to form 
several embryo cells for the corresponding number of new cells. The old cell either dies or enters into the encysted state. After an uncertain period the cell wall bursts, and liberates the new ones. The mother cell, containing the danghter cells, may remain encysted for an indefinite
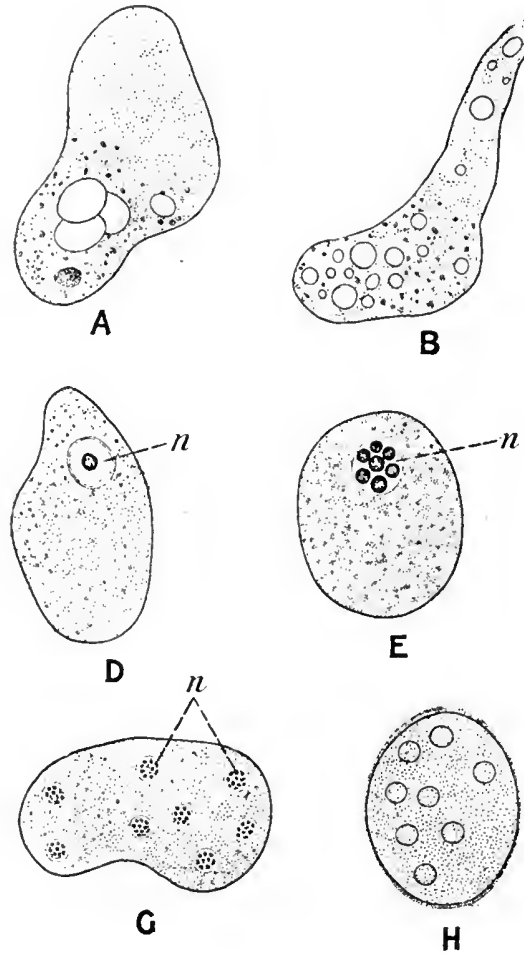

Fig. 101.
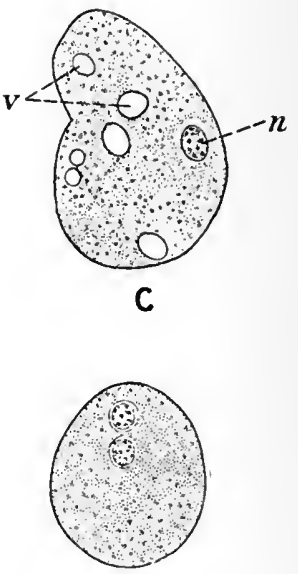

$\mathbf{F}$

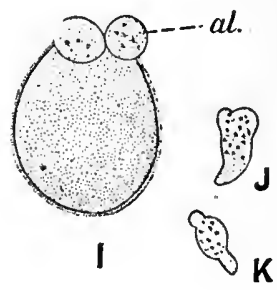

Amoeba Cell.

$A$ and $B$, living amoebae, showing changes of form and vacuolation in the protoplasm: C, D, E, amoebae, showing different conditions of the nucleus (a) ; F, a specimen with two nuclei, preparing for fission; G, a specimen with eight nuclei preparing for multiple fission; $H$, an encysted amoeba containing eight nuclei; I, a cyst from which young amoebae (a1) are escaping; J, K, young amoeba free.

After Casagrandi and Barbagallo from Allbutt's System of Medicine. 
time. In this state it is much smaller than the ameboid form, and is non-pathogenic.

There are two well recognized species of amebae, the kind above described, and the amoeba coli mitis (Fig. 101) which is occasionally found in healthy persons. This organism is also found in other bowel affections. It is nonphagocytic, twelve to thirty-six microns in size. Propagation is by gemmation or budding; a portion of the cell body being thrown out and then broken off, forming $a$ new individual.*

All authorties now agree that the bacteria of symbiosis. and other associated micro-organisms, have much to do with the pathogenicity of the amebae.

I have noted that the cases presenting themselves during the summer or autumn usually show the more active and phagocytic amebae, or, more properly speaking, in those cases in which I have found the more active and phagocytic amebae, I have also found the greater virulence. In making microscopic examinations of most cases the parasites are either very inactive or cease motility quickly, rendering necessary at times two or three examinations to make a positive diagnosis. In most cases the bacteria of symbiosis are quite numerous.

The amebae are introduced into the intestinal tract through the mouth and stomach, but the acid gastric

*After close observation, covering a great number of cases, the writer has become convinced that there exists a pathogenic ameba which does not correspond exactly with the description above given of the ameba hystolitica.

This ameba is smaller, the hyaloplasm is not so distinct though its lighter zone is discernable and this hyloplasm or ectosarc can be seen forming pseudopods. This ameba is both granular and phagocytic, and is often observed very active, hence in the writer's opinion, this ameba likewise is pathogenic. 
juices prevent their propagation. They pass on into the colon to gain lodgment at favorite points, namely, the iliocecal valve, hepatic and splenic flexures, and especially upon the plicae transversalis recti. In most cases the inflammation begins first in the rectum and extends upward by continuity.

The writer has endeavored to explain the periods of exacerbation and amelioration of symptoms, in the following ways:

First.-The entamoeba hystolitica is especially fond of feeding on the juicy sub-epithelial structures, and, in a given case, this particular crop or generation, within the plentiful surroundings, may become indolent and easily satisfied, and also less active in the process of sporulation.

Second.-The parasite may be in a state of encystation, during which period the amebae remain dormant or nonpathogenic until finally a different generation produces a more active and phagocytic type.

Third.-Because of the presence of a greater or less number of bacteria of symbiosis which, in the light of observation of most authorties, seem essential to the activity and virulency of the amebae.

A further study of the problems of immunity may in the future yield information which will be of paramount importance in amebic dysentery, both in reference to the amoeba and the symbiotic bacteria.

This disease is most often contracted through drinking water.

Flies and other insects are possible means of transmission.

It can also be developed through contact. 
When making a microscopical examination of the feces for amebae, the following will be helpful to the inexperienced microscopist: Technique: Warm the slide slightly. Secure a small bit of the mucus from the stool and place upon the slide. Cover with a cover glass quickly, and press it gently until the material is thinly distributed. Examine at once with the one-sixtl or the oil immersion objective. This should be done as rapidly as possible since the amebae retain motility for only a short time in temperatures, much lower than body heat. If now they can not be found, apply warmth by holding an electric light bulb to one side of the stage. They may then be seen. Never be positive that the amebae are not present thongh not found. They may be in a state of encystation in the tissues, and only after an acute exacerbation of the disease, will they be found.

A still better plan, and the only accurate way, is to examine the scrapings of the ulcerated mucous membrane. This method should always be practiced when possible.

The most important of the associated organisms, are the streptococcus, staphylococcus, bacillus coli communis, trichomonas intestinalis, paramoecium, cercomonas intestinalis, lambia intestinalis, bacillus pyocyaneus, and others.

Pathology.-Pathological lesions are almost always confined to the rectum and colon, but occasionally the ileum may become involved.

Appendicitis is quite common.

The mucosa appears red and congested, and covered with mucus usually tinged with blood.

The infiltration and edema now invade the submucosa, necrosis of the overlying mucous membrane takes place, 
and the amebic ulcer is formed. 'This necrosed area may be oval or irregular in shape and appears to project over the level of the mucosa.

The amebae gain access into the sub-mucosa through the inter-glandular spaces and carry with them the asso-

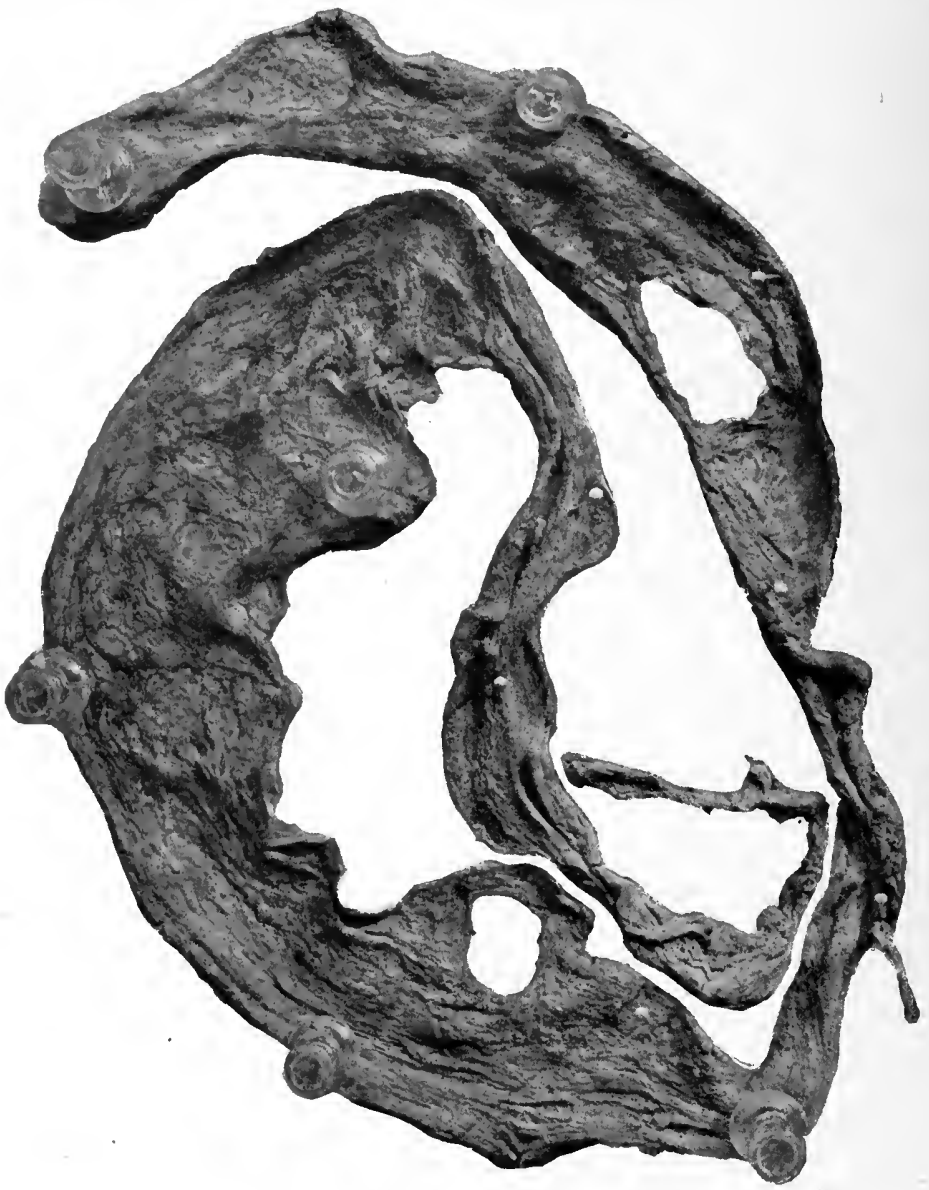

Fig. 102 .

Slough of Mucous Membrane Twenty-eight Inches in Length from a Fatal Case of Dysentery.

.. Photograph of specimen from one of Jelk's cases. 
ciated organisms. Here they set up an active inflammation, and produce ecchymosis and swelling of the glands. 'The number of the amebae in the sub-mucosa is great, since they prefer this juicy sub-epithelial tissne, no doubt because they find food more easily. When they get into the sub-mucosa their presence excites a reactive inflammation at once.

It is important to note here that the bacteria of symbiosis play a very important part in the inflammation just described. Necrosis now takes place in the inflamnatory area and sloughing follows. In grave and fatal cases this undermining process, so to speak, may become so extensive, and the congestion so great that large areas will neerose and slough. 'The writer has preserved one specimen of this character twenty-eight inches in length. (Fig. 102).

The muscular coat of the bowel offers greater resistance to the amebae, so they seldom invade it. Occasionally, however, this undermining process will extend into the intermuscular tissne, and produce the same results as before described. In this way the larger and deeper ulcers form. (Fig. 103).

The involvement of the rectum in one case was so extensive that the new scar-tissue produced an almost complete stenosis. Higher up the nlcerations usually cover a smaller area. A sharp-edged, clean-cut ulcer results. This ulcer may involve the greater portion of the thickness of the wall of the bowel, but the undermining is not so extensive and the thickening which results lower down is not so marked here. One post-mortem revealed nine distinct perforations in the splenic flexure, which pro- 
duced sudden death when the loose attachment of the omentum was broken by gaseous over-distension.

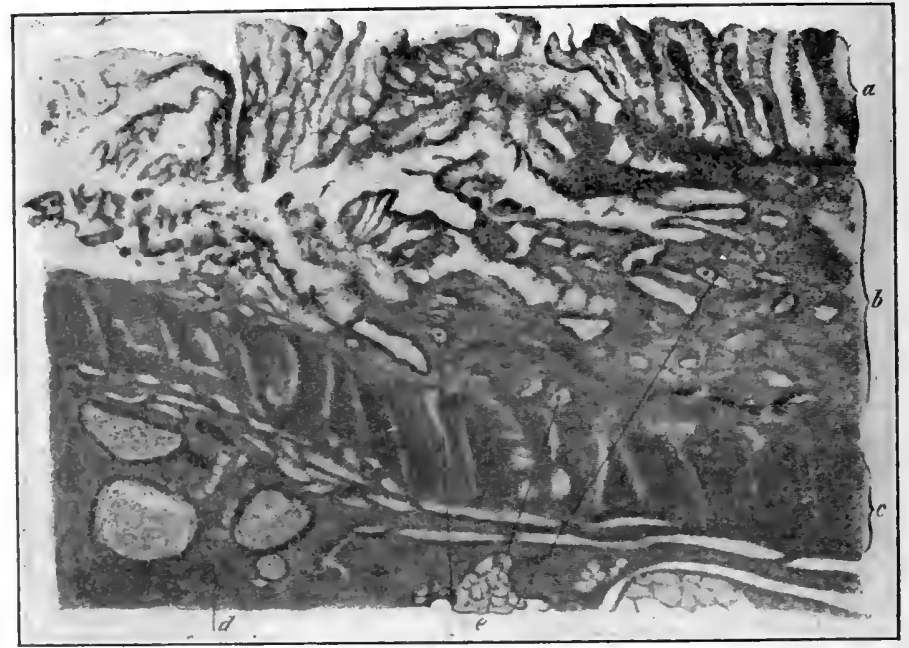

Fig. 103.

Edge of Intestinal Ulcer.

('Toluidin-blue and eosin. Beck 1 inch, Oc. 3.)

A. Mucous coat which projects over ulcer at $f$.

B. Submucosa.

C. Circular layer or muscle fibres.

$D$. Tissues of mesocolon.

E. Amoebae in dilated lymph spaces.

Courtesy of Dr. H. F. Harris, Atlanta, Ga.

The writer wishes also to call attention to certain spots and lines which he considers almost diagnostic when present. By careful examinations with the proctoscope small red papular spots may be seen dotted about among the already well defined ulcers. Perhaps on the following day the red spots will show a little white or yellow point of necrosis in its center. Upon the next examination an ulcer will be seen to have taken its place. 
In another instance a few circinate or ring-worm-like lines in the mucosa, a picture which is not observed in other forms of intestinal infection, will be seen. These

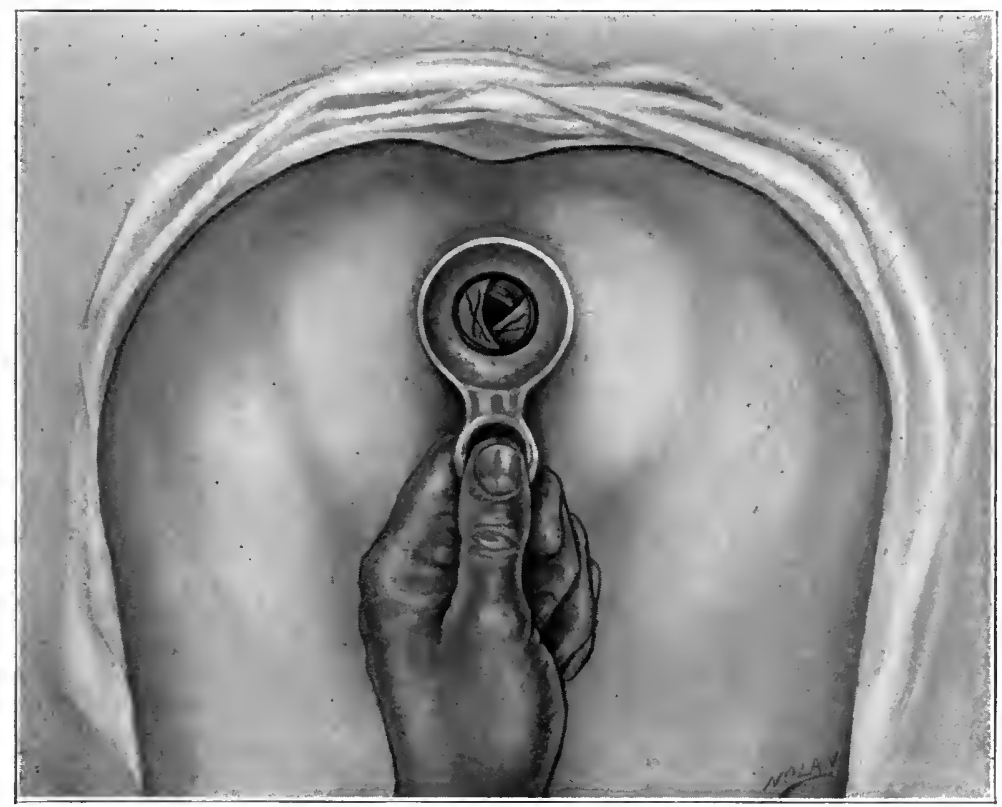

Fig. 104.

Dysenteric Ulceration on the Valves of Houston. (After Tuttle.)

lines or ulcers are chiefly submucous, but sooner or later break into the undermined ulcer, and may then assume any shape. New lines will form, however, to tell the story. (Fig. 104).

The writer has also observed small openings at points along the courses of these circinate lines leading to extensive submucous ulcers. At other times the intestinal mucosa presents only a few circinate lines overlying the 
sub-epithelial ulcers, while the remaining mucosa presents a red granular appearance.

In a few cases (unmistakably amebic) the disease appeared to be only a lypertrophic proctitis, or a proctosigmoiditis, and in others the mucosa appeared puffy or edematous.

It is very probable in my opinion that some of these conditions were concomitant and due to associated conditions, especially collateral infections.

Amebae have been found free in the peritoneal cavity, and in other parts of the body, especially the liver. Here, when unassociated with collateral organisms the parasites are non-pyogenic. A true amebic, unassociated infection in the liver would mean simply that; and not an abscess carity filled with the most offensive pus, as is so often found. Perhaps in almost all cases amebae have heen convered into the liver, and, but for the fact that they were unassociated with pyogenic organisms, abscesses would surely follow. Hepatic abscess complicates probably twenty per cent of all chronic amebic infections, lowerer, exact statistics can not be obtained.

Councilman found this complication in six out of eight antopsies.

Strong and Musgrave in fourteen out of ninety-seven autopsies.

Ont of a series of twenty-five cases treated by the writer in 1908, four were complicated by hepatic infections. In two of these cases the diagnoses were verified by operations. In one a large abscess of the right lobe was found, and in the other, the right lobe was inflamed and firmly arlierent to the omentum and hepatic flexure of the 


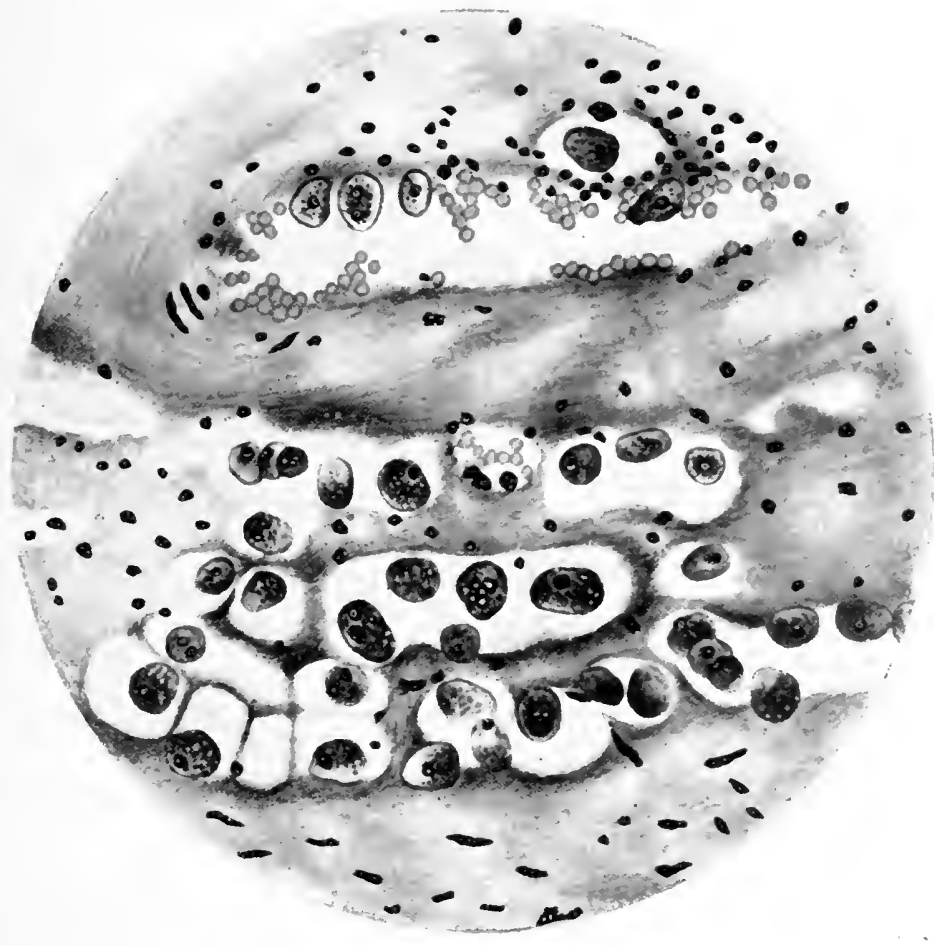

PLATE İI.

Section of intestine just below ulceration. Toluidin-blue and eosin. Beck $1 / 4$, Oc. 3. In upper portion of the field a large vein is seen; the wall of the vessel which is nearest the ulceration is being infiltrated with small cells and is breaking down; both red and white cells and amoebae are seen within the lumen of the vessel. In the lower portion of the field many amoebae are seen, some in the tissues, and others in the lymph spaces and lymph channel. 
$\therefore$ 
colon.: A cholecystitis was also present, and required drainage for six weeks.

'The infections may be carried into the liver in two ways :

First, and most probable, through the portal vein, which has often been found infected. (See Plate II.)

Second, by transmission through the intestinal wall.

Craig claims that the kidneys often present the lesions of acute parenchymatous nephritis.

Symptoms.-In the more virulent or malignant cases the onset is usually sudden, and may or may not be ushered in with a rigor. The attack is preceded by a period of malaise, often accompanied by constipation. An attack of acute indigestion often precedes this form of dysentery. The patient may have six to forty bowel movements during the first 24 hours, usually sero-sanguineous in character. Prostration is early. By the second or third day considerable blood and pus begin to appear, the latter being very offensive in odor. Prostration increases with the further absorption of toxins. Temperature usually rises to 102 to 103 degrees F., and is of the irregular remittent type. Delirium may be pronounced. General abdominal pain and tenesmus with tympanites and tormina are prominent. The facies abdominalis denote suffering and anxiety. The nose is pinched and the upper lip is retracted, and the condition now is a grave one. The thighs are flexed upon the abdomen and legs upon the thighs in such manner as to relieve pressure upon the abdominal viscera. Considerable tenesmus precedes and accompanies all bowel movements and may follow for several minutes, though as a rule a greater or 
less relief follows the passage of only a small amount of bloody mucus. Later the more offensive discharges, containing greater quantities of mucus, pus and blood, with perhaps muco-fibrinous casts, or mucous membrane sloughs, indicate necrosis.

The above symptoms are soon followed by delirium, subnormal temperature, rapid, feeble pulse, clammy perspiration, glazed skin, collapse and deatl. If, after the sloughs are passed the patient survives the sepsis and toxaemia, and healing of the ulcers follows, the process is a slow one. These ulcers are finally filled with granulation tissue and fibrinous material, which contract, causing more or less stenosis. 'The symptoms of sepsis and toxacmia from the absorption of necrotic material and toxins very gradually diminish until the patient is able to resume his regular oceupation.

The following arse reports will be helpful:

Case 1.-Name, Dr. _ ; age, 36 years; race, white; occupation, physician; family history, negative; previous state of health, good, until six months previous, during which time he suffered a rapid decline. Symptoms: Lost thirty or forty pounds in weight; complained of slight colicky pains over course of colon; troubled with loose fermentative diarrhoea; inactive liver; coated tongue; temperature $992-5 \mathrm{~F}$; pulse, 60 ; skin, dry and muddr: slight tenderness on pressure over cecum, hepatic and sigmoid flexures; pronounced melancholia, insomnia, and malaise were present. Had not noticed passages of mucus from bowel but spoke of a very offensive odor. Proctoscopy revealed a considerable quantity of sanguino-purulent mucus in the rectum, and the rectal mu- 
cosa was covered with same, mixed with some light brown fecal material. Small circinate lines and punctate ulcers were seen on the rectal walls and valves of Houston. A mild gramular procto-sigmoiditis was noted. Microscopic examination revealed entamoebae histolyticae, phagocytic and associated with bacteria of symbiosis, trichomonas intestinalis, paramoecia, and others. Diag. nosis, Amebic Dysentery.

Case 2.-Name, Dr. ; age, 53 years; race, white; occupation, physician; family history, negative; previous state of health, good, until 23 years of age, since which time he has never been well. Symptoms: At the age of 23 suffered a very severe attack of dysentery and for a long time, hope of recovery was despaired of. Later a change of climate seemed to contribute to his slow but apparent recovery. After returning home suffered a relapse. Since that time has suffered abatement and acceleration of symptoms; alternating attacks of diarrhoea and constipation; suffering now from profound melancholia and insomnia with suicidal inclinations. Temperature, sub-normal; pulse, 65; tongue, dry and coated heavily, round and thick; skin, inactive and muddy; liver, enlarged, extending three inches below costal border and tender, probably the seat of a large abscess. Pain on pressure over entire course of colon, especially over caecum, hepatic and sigmoid flexures. Furunculosis (Staphylococcic) over entire body ; atonia gastrica with dilatation; kindeys, normal.

Doctor William Krauss' report: “Proctoscopy-rectal walls very much thickened, scarred and stenosed, this latter condition observed at recto-sigmoid juncture 
also; red granular hypertrophic recto-sigmoiditis: . The characteristic nlcers, previously referred to, were found beneath a coating of offensive blood-tinged mucus, which was mixed with pus. Microscopic examination revealed large active phagocytic amebae histolyticae, colon bacilli, trichomonas intestinalis, cercomonas intestinalis, and other symbiotic bodies in great numbers. The blood cxamination, made by Dr. Krauss in this case shows the following: $3,940,000$ red cells, 75 per cent hemoglobin, 13,700 white cells, of which 74 per cent polynuclears and 3.3 per' cent eosinophiles. The opsonic index failed. The bacteria isolated from the pustules were staphylococcus albus and a single colony of aureus. I regard the blood condition to be one of secondary anemia witlı mild coceus infection, and the moderate eosinophilia is probably due to the intestinal condition."

The furuncles were healing nicely when I last saw the patient, and he expressed himself as feeling greatly improved.

Diagnosis, Amebic Dysentery.

'The writer looks with suspicion upon any case of dysentery or diarrhoea, reeurring or relapsing, which has failed to respond promptly to treatment.

Dysentery and diarrhoea are not essential symptoms of the existence of amebiasis, though this is contrary to the generally accepted theory. In many cases the patient will complain of recurring diarrhoea which has existed for months or years. These attacks are accompanied by passages of mueus, usually considerable in quantity, and occasionally stained with blood. The patient complains of almost constant pain or discomfort in the left iliac 
fossa, and, when the lower rectum is the seat of considerable ulceration, pain at the end of the spine and in the rectum is felt. This symptom is momentarily relieved by evacuations:

A case from the Mississippi Delta, reported by me to the American Proctologic Society, had most violent symptoms from the onset. On the fifth day a large slough of mucous membrane (see Fig. 102) was passed en masse Thirty-six hours later the patient died.

Most of the chronic cases will give a history of having lost much weight, perhaps twenty to fifty pounds. Many have symptoms of interest to the stomach specialist, and to the nemrologist.

Complications and Sequelae.-These are very numerous indeed.

Of 1537 cases of diarrhoea in Fgypt only 406 were uncomplicated.

Hepatic abscesses were found in six out of eight autopsies by Councilman. In four of these they were multiple.

Strong and Musgrave found hepatic abscesses in 14 out of 97 cases. The writer, as previously stated, found liver infections in four out of twenty-five cases.

The vermiform appendix lias been found to be involved in fully ten per cent of chronic cases by the writer.

Among the other complications most frequently occurring are, perforations, extensive slonghs, hemorrhages, fibrosis of the valves of Houston, rectal stenosis, adenomata recti, cholecystitis and jaundice, peri-rectal abscess, hemorrhoids, fistula, pneumonia, pulmonary abscess, plemisy, bronchitis, nephritis, portal thrombosis, cerebral and meningeal emboli, gastritis, atonia gastrica, mel- 
ancholia, which is often profound, and in two recently treated cases extensive skin lesions were encountered. In one of these a microscopic examination revealed the presence of the staphyloccoccus albus and aureus.

Statistics from all sources show that perhaps twenty per cent of all cases are complicated by hepatic infection. 'The right lobe is most of ten involved.

Perforations may occur along the course of the colon at any point between the rectum and appendix. Perforative appendicitis has been noted.

Perforation occurred in 85 out of 580 cases selected by Beranger and Feraud.

Stenoses have been observed in a large per cent of chronic cases, usually in the rectum and sigmoid. When fibrosis of the rectal valves is observed, it is a grave obstacle to the complete cure owing to interference with drainage and local treatment:

Hemorrhoids, though frequently noted, are not serious complications as a rule.

'The other complications mentioned above should be borne in mind and treated when they occur.

Diagnosis.-This is rendered easy by means of the microscope, all doubt being removed by finding the entamoebae histolyticae in the stools, or in the material curetted from the ulcers in the rectum and sigmoid.

Prognosis.--The prognosis in amebic dysentery is likewise much graver than in the acute catarrhal form. It may be said to depend upon several things: First-The previous state of health of the patient. Second-The hygienic condition of the patient's surroundings. ThirdThe efficiency of the treatment employed. 
In the United States the total number of deaths from all forms of dysentery in 1850 was 20,556 , a per cent of 6.32 of the total mortality.

In 1880 out of 756,893 deaths, 10,825 were from dysentery.

Treatment.-The treatment of dysentery will be discussed under the heads, (a) Prophylactic. (b) Dietetic. (c) Remedial and Operative.

Prophylactic.-Strict attention should at all times be given to the hygienic condition of surroundings. Remove and avoid as far as possible the causes of dysentery. Cases should be isolated when it is possible to do so. All excreta should be carefully disinfected and deposited where the water supply will not be contaminated. The country practitioner, living where there is no sewerage system, should never neglect to caution those attending the patient to deposit the excreta in a hole dug for the purpose as far removed from the water source and garden as possible, after first disinfecting thoroughly.

If a person, knowing the danger, were to deposit the excreta of a dysenteric patient in a garden, it would be inexcusable. The writer has, however, seen this done by some who had never suspected danger in so doing. In the country, and in small towns, without sewerage, little closets are usually found in or near the gardens, and are often made sources of fertilizing material for the growth of vegetables. It is the duty of the physician to educate his patients in regard to all dangers resulting from such gross unsanitary pratices. Wells and cisterns are contaminated much more often than the average layman suspects. When the source of the drinking supply is 
at all questionable, the water should be boiled before drinking.

Over-crowding and poor ventilation should be prevented. The care of the room occupied by the patient is important. Unnecessary furniture, such as curtains, rugs, carpets, etc., should be removed. Disinfectants should be used at regular intervals. Linen should be clanged daily. Bed pans, commodes, drinking cups, etc., should be disinfected thoroughly.

Diet.-Diet is as important as any other matter in the treatment of dysentery. During the period of acute intestinal symptoms it should consist of boiled, or perhaps better still, peptonized sweet milk, buttermilk, whey, egg whites, light animal broths, peptonized beef juice, barley water, and perthaps one of the standard malted milk foods for infants.

In all cases select a diet which is digested as far as possible in the stomach, and which has little waste. Food is best given at intervals of one to two hours in acute cases. Plain sweet milk may be diluted with barley or rice water, lime water or Vichy, if imperfectly digested.

During convalescence in all forms of dysentery and for chronic cases, the writer prefers butter milk, peptonized or pasteurized sweet milk, whey, and eggs. In some cases tender portions of turnip tops, mustard, and spinach have been given, and were relished by the patient. It is, however, questionable as to the propriety of giving the patient much vegetable diet.

In cases of amebic dysentery the writer is especially partial to a diet of milk and egg whites. The eggs may at times be prescribed in large quantities, from eight to. 
fifteen pér day. 'They can be ordered raw, mixed with milk, or in the form of egg-albumen. The latter is made by stirring the white of one egg into a glass half full of crushed ice, then flavor with orange juice and sweeten. Diffusible stimulants, such as champagne, sherry wine, or whisky, may also be added to the egg mixture when cardiac weakness and adynamia are present.

The albumen may also be mixed with sweet milk, or sweet milk with lime water in the form of a milk-shake, to which may be added the alcoholic stimulants, if indications exist.

Butter-milk is an especially favorite diet. Its acid properties make it desirable.

The articles of dict which are contra-indicated are all dishes highly seasoned with pepper, cinnamon, nutmeg, etc. Vegetables, especially the raw varieties, pork, salt meats, veal and fish, saccharine foods, and fried foods, nuts, oatmeal and fruits, should also be interdicted.

Remedial. The medicinal treatment of dysentery is a most interesting subject. A great number of so-called specifies, and much praised remedies, have been handed down to us, but most of them have proven so msatisfactory that it is no surprise that most of the present day suggestions are greeted with a certain amount of skepticism or personal prejudice. The systemic treatment as a cure for dysentery is erroneous. It is a local disease and therefore requires local treatment. This is certainly true with reference to immediate pathology, but other remote pathological conditions may require constitutional treatment.

The ameba is a very low form of organic life and is very easily killed or rendered inert. The fact remains, 
however, that the parasites are embedded in the tissues in such vast numbers as to make their destruction difficult. Certainly any chemical which is given by mouth, after passing through the stomach and small intestines, can possess little parasitic effect when it reaches the lower colon, sigmoid flexure, and rectum. Therefore, our chief reliance must be placed in local applications, which are used for the following purposes, namely: That of washing away the pus, mucus, and debris, and, at the same time, the amehae and other pathogenic organisms. Also that of antisepticizing the bowel contents and walls, that the further growth and development of the pathogenic organisms will be imlibited.

It is also important to remember that the remedies selected should be those which will destroy the greatest number of organisms beneath the lining membrane of the bowel without destruction to the tissues themselves.

In the earlier stages of acute dysentery the patient should be put in bed and absolute quiet enjoined. Chilling draughts of air are to be cantionsly avoided since they are apt to increase the congestion of blood toward the internal viscera. Bathing the patient with warm water, vinegar, or alcohol will often give great comfort hy relieving the burning sensation in the skin. The perianal region should be sponged frequently with an antiseptic wash, such as a mild boric acid and formalin solution, and dusted with some mild antiseptic powder, as equal parts of boracic acid and aristol. An ointment of similar composition may be used instead. Application of lot or cold to the anal region will often relieve the burning and tenesmus in the lower rectum. 'The hot hip 
baths also have been very helpful in relieving this condition.

In the more severe cases the constant application of ice bags over the left iliac region gives comfort. Hot fomentations are sometimes to be preferred but in the majority of cases, the ice bag is better.

The severe griping and tormina are relieved quite readily by hot turpentine stupes or by large flax seed meal poultices. These may be used just as frequently and for as long a period as needed.

Laxatives.-Occasionally absolute rest and strict diet are all that is needed to relieve the patient, but it is in most cases best to administer some mild laxative to remove the contents of the bowel, which act as both mechanical and chemical irritants.

Castor oil and magnesimm sulphate, to the latter of which may be added dilute sulphuric acid, are the most popular remedies for this purpose. The salines, by their hydrogogic action deplete the infiamed mucosa and wash away many of the infecting micro-organisms. It must be remembered, however, that all purgatives act as irritants to the intestinal mucous membrane in a greater or less degree, and their use must be guarded with judgment. In some cases they would be harmful. If there has been much diarrhoea and the stools are copions and thin, purgatives are contra-indicated.

When to repeat a purgative is another question that should be considered with care. Often much harm is done in this way.

Calomel, or calomel with ipecac, is often ordered in small doses for a dry, furred tongue, and inactive liver 
with foamy acrid discharges. Our aim in giving calomel is not only that of producing the antiseptic action of bile, but also by depleting the liver, to relieving the portal congestion; and this in turn, the congestion of the veins about the rectum. 'The severe griping pains and tenesmus, the diarroloea, and restless condition of the patient, when present, must be relieved or the outcome will be rapidly advelse. Opium is the remedy, either in the form of Dover's powders, paregoric, laudanum, or morphine. This last is no doubt the most popnlar form of the drug and is best used hypodermicaliy. The dose should be just large enough to keep the patient quiet and to relieve the suffering but never sufficient to produce narcotism.

It must not be forgotten that opium may do great harm in some instances. If nature is attempting to throw off the putrid contents of the bowels in large, liquid stools, we should not give opium, for in so doing we are interfering with her efforts to relieve the condition.

A large number of intestinal antiseptics lave been given internally for dysentery, the principal ones being ealomel, lead acetate, zine sulphocarbolate (in one-half to three-grain (loses), salol, guaiacol carbonate, bichloride of mercury (dose, grains 1-120-1-50), and acetozone. 'These are all, howerer, given by the writer with a certain feeling of uncertainty.

Those arses which begin with symptoms of cholera morbus, with nansea and vomiting, and subnormal temperature, call for hypodermic injections of morphine sulph. gre. 1/s, and atropine sulph. gr. 1-150. To control nansea may be given acid carbolic and tr. iodine, of each one minim well diluted, by mouth. This is followed by 
calomel, grs. 1/s-1/4 and salol, grs. 2 to 5 , with just a sufficient amount of hot water to administer same.

In other cases cocaine hydrochlorate (gr. 1/8-1/2) may be given, and, where there is much depression, warm enemas of normal salt solution may be given, or this may be given by hypodermoclysis. 'The effect is a dilution of the toxines and a reaction. A mustard plaster or hot turpentine stupes over the epigastrium are beneficial in these cases. If the temperature and pulse are not subnormal, the tormina, tenesmus, and burning can be allayed by enemas of cold water, the temperature of which should be regulated to suit the case.

When there is marked irritability of the rectum, the following suppository should be inserted before injections are made:

If Cocaine hydrochlorate, Ext. stranonium, Ext. belladonna-each gr. ss.

Ol. theobromae-Q. S.

M. et. ft. in suppositories No. 1.

Sig.-Hold the suppository in the anal canal about one minute, then press into the rectum with the index finger.

Kartulis claims that he found ipecacuanha to have an almost specific influence upon dỵsentery.

His method of administering this drug was to give a one-half grain injection of morphine hypodermically and place a mustard plaster or turpentine stupe over the epigastrimm. After half an hour twenty grains of pulv. ipecac was given, and this dose was repeated every halfhour to one hour until an onnce had been given. 
Another method of giving this drug: Put 2 to 8 grams ( $1 \%$ to 2 drachms) in 500 grams (1 pint) of water and let stand two lours. This solution is filtered off and constitutes the first dose, or this is at times divided into two or more doses. According to Kartulis, this always produces emesis and diarrhoea, but, after a second or third infusion, which is made from the remaining portion of the powder with the same quantity of water, lias been taken, the vomiting and purging become less frequent.

If, after the third day's treatment with these infusions, the patient has not improved, another series of infusions with a fresh supply of ipecac should be given.

The writer has mentioned this treatment only to condemn it. It has been known to produce death, and does

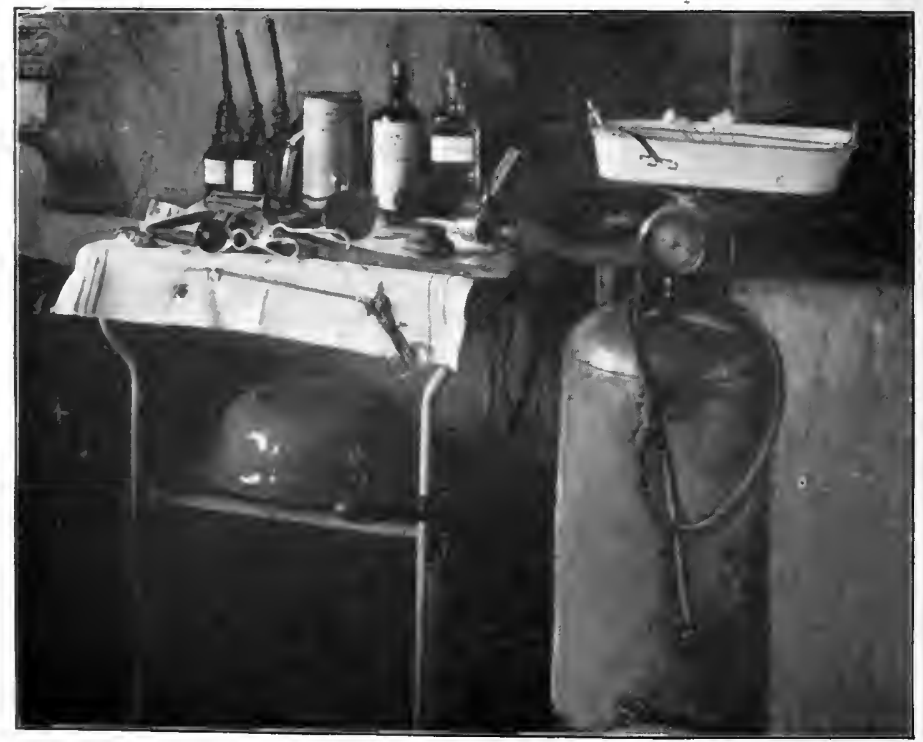

Fig. 105.

Instruments, Etc., Required in the Office Treatment of Dysentery. 
not cure the disease. In all cases it is a cardiac depressant and lowers the physical resistance of the patient. It is a violent intestinal irritant. The powdered drug has also been found impacted in fatal perforating ulcers of the bowel. To my mind, therefore, its administration in this disease, by this method, is dangerous, adding insult to injury.

For the acute catarhal type the elimination of irritating substances and free exosmosis obtainable by the administration of epsom salts, and enjoined rest in bed, with abstinence from all but the blandest forms of diet, will often suffice. In these cases, howerer, the injection of tepid water, containing to each quart, gtts. $\mathrm{x}$ to $\mathrm{xx}$ of formalin, and 1 tablespoonful of boric acid, may be necessary. This is often followed by the same quantity of cold water, or by the injection of 1 or 2 ounces of olive oil and one scruple of bismuth subnitrate. These injections can do no harm and are surely destructive to the life and propagation and pathoganic properties of the infecting agent.

If the symptoms do not abate, and the patient does not obtain marked relief within the first few days of the use of the above described treatments, pathology may be present which may require other forms of local treatment in the nature of topical applications.

A subacute catarihal condition may supervene in which an astringent and antiseptic treatment will be required to complete a cure. For this purpose may be used the injection of a tannic acid solution, one drachm to a pint of water, followed by the introduction of a suppository containing : 
If Fxt. belladoma _...

Ext. stramonium _..._........ grs. 1/2

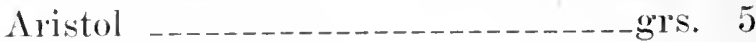

Ol. theobroma, Q. S.

M. et. ft. in suppository, No. 1.

Or the following ointment:

Ii Ext. helladonna _._._.

Ext. stramonium _..._........-gis. 1/2

Aristol _...............

Liquid albolene, Q. S.

M. et. ft. in ointment.

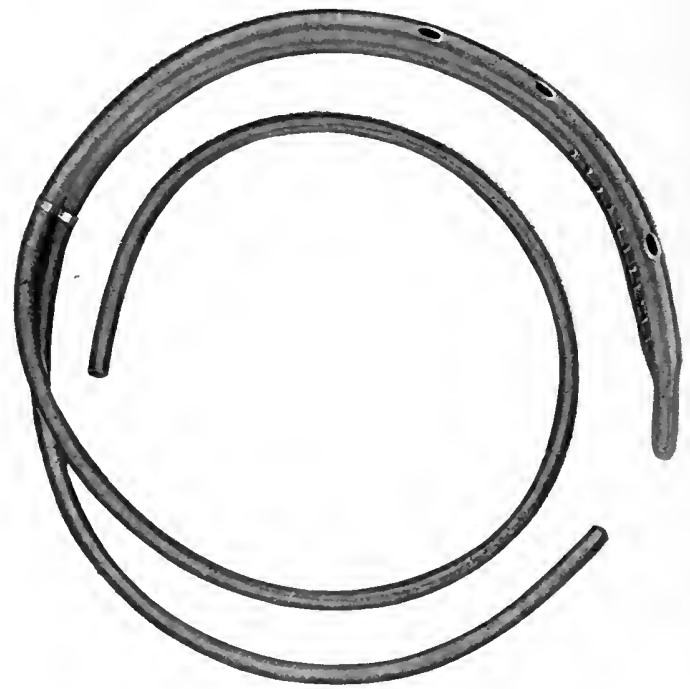

Fig. 106.

The Jelks Soft Rubber Recurrent Recto-Colonic Irrigating Tube.

Courtesy of Dutro \& Hewitt, Memphis, Tenn.

If the disease assumes one of the more virulent types, and if the ulceration is extensive, still more radical measures slould be songht in the high irrigation with the formalin boric solutions. 'These, if possible, should be given 
through a recurrent tube (Fig. 106), since by this means only can a large quantity of the solution be used without distending the inflamed and ulcerated bowel to a painful or perhaps dangerous degree.

Four to eight quarts of this solution are usmally required for one irrigation.

Some authorities are partial to the use of quinine solutions (1-5000 to 1-500) in cases of amebic infections. Among the advocates of this drug are Musgrave and Strong, and Osler. H. F. Harris, of Atlanta, says: "I used this treatment with great persistence in some of my earlier cases, but not in a single instance was there the slightest perceptible result."'

"Injections of 1-100 to 1-300 watery solution of bi-sulphate of quinine were somewhat beneficial in one or two instances."

My own experience with these injections is in accord with that of Dr. Harris.

The use of formalin solutions in the strength of 1-500 to 1-1000 liave in the writer's hands afforded the best results.

My study of the effects of this chemical has extended over a period of ten years. I have relied not only upon clinical results obtained, but also upon the microscopical observations in demonstrating the efficiency of formalin. After only one or two injections with these solutions I lave been unable to find any living organisms in the bowels for hour's afterwards.

'This it was observed was not the case when other solutions were used. 
Rapid healing of the ulcers was always noted while continuing the irrigations of the formalin in the above mentioned strengths.

'T'o be certain of the effect of this drug, its use was discontinued for the time being, and such irrigations as plain water (warm or iced), normal salt, and quinine solutions, were substituted. In every instance the ulcers reformed, and both amebae and bacteria of symbiosis were found again in the microscopical examinations. Upon returning, however, to the formalin irrigations these micro-organisms disappeared and the ulcers began the process of repair. Thus the writer las conchuded that this chemical, judiciously used, is really the most effective in the destruction of the amebae and associated

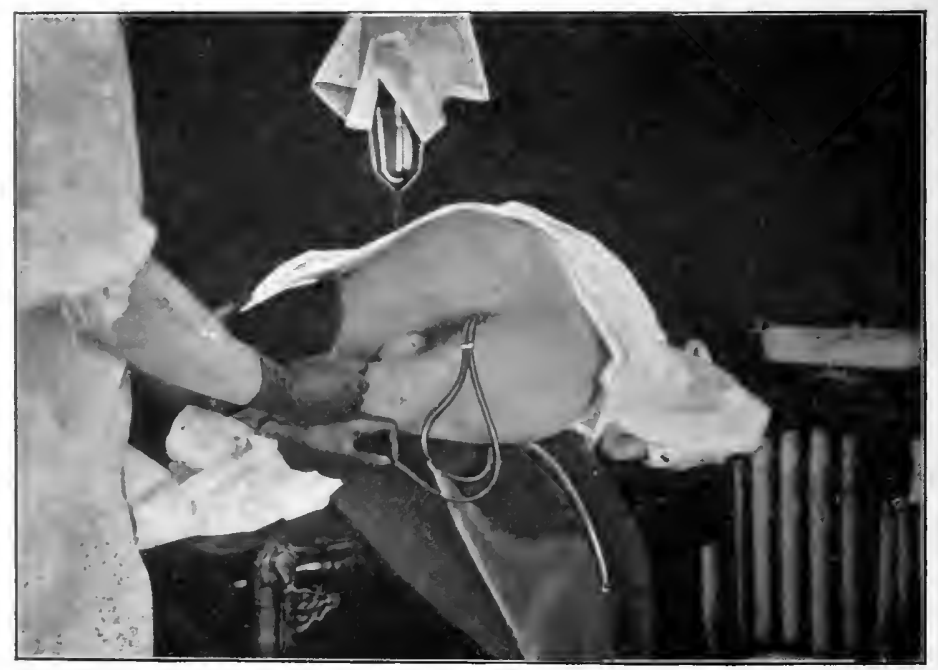

Fig. 107.

Exaggerated Sims' Position Showing Method of High Irrigation of Colon Through Jelks' Recurrent Tube. 
organisms, and most valuable in the treatment of dysentery.

The injection of olive oil and bismuth almost instantiy relieves the painful effects of these solutions.

The dangers of over-distension of an inflamed and ulcerated colon are difficult to over-estimate. To avoid this the writer has devised a double or recurrent colon tube, made of soft rubber, and constructed in such man. ner as to facilitate its introduction through the rectum, into the sigmoid and descending colon. The tube, having been properly inserted, it is an easy matter to change the position of the patient, and by so doing irrigate the entire colon. (Fig. 107).

In some instances the tube is obstructed by the rectal or recto-sigmoid valves, which may necessitate its introduction through the sigmoidoscope or proctoscope. In chronic cases especially has this difficulty been encountered, since in these a fibrinous infiltration of these structures often exists, rendering"almost impossible the use of an ordinary rectal tube. To ascertain whether or not the tube has coiled in the rectum, the operator can introduce the index finger, well annointed, with the lubricant given below. After several unsuccessful attempts have been made the proctoscope should be introduced and the tube inserted through it, as is shown in Fig. 108.

A lubricant of the following formula is preferred by the rriter:

T. Tragacanth Powder (Best) _-_-_-grs. 384

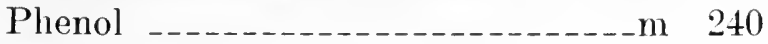

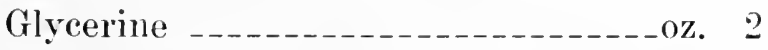
Aqua Dist _..._._._._. S. ad. qt. 1 
M. Sig.-Shake up gum with enough alcohol to make thick paste. Add acid and glycerine. Shake well and add water all at once. Agitate vigorously.

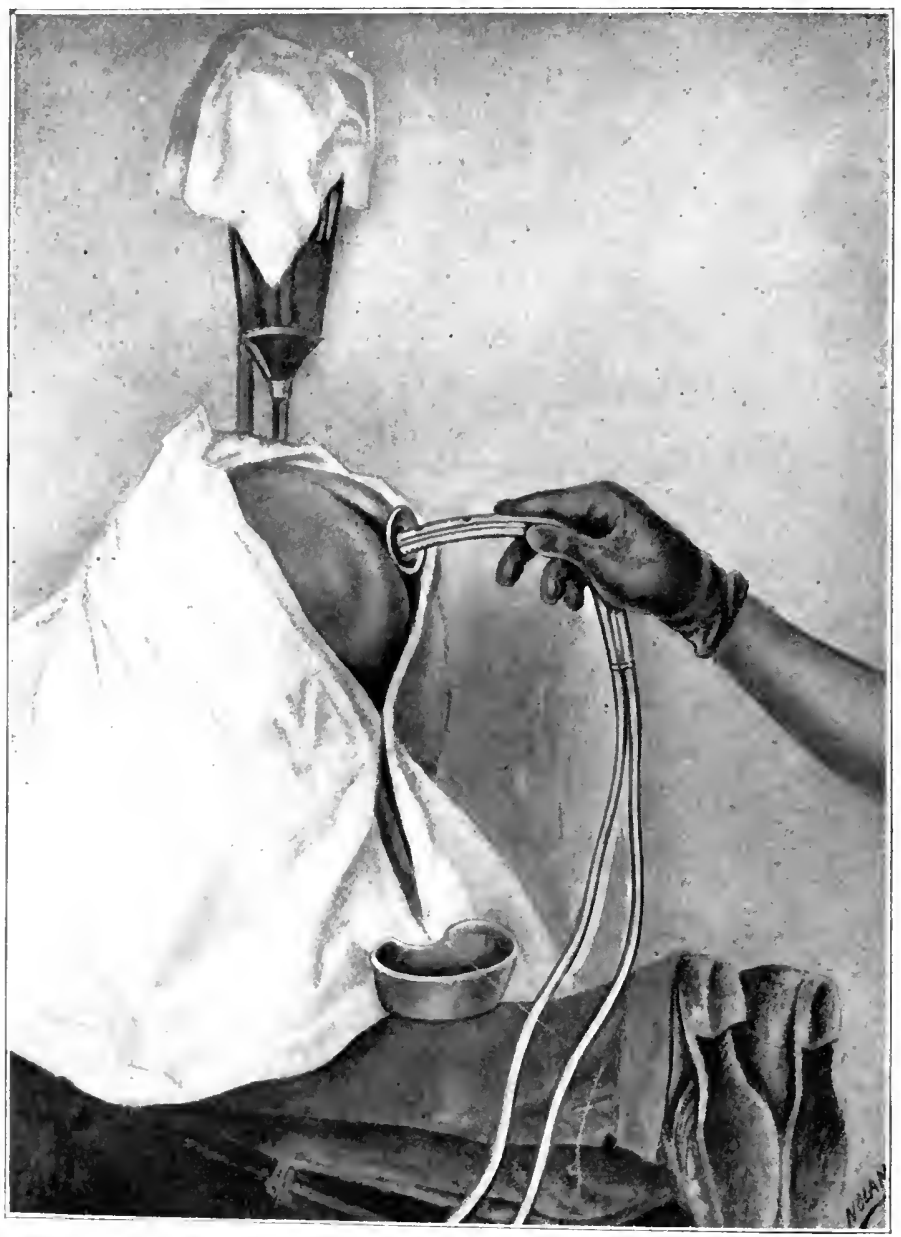

Fig. 108.

Position of Patient for Proctoscopy, Proctoscope Introduced to Facilitate the Introduction of the Colon Tube. 
Dr. Louis LeRoy, of Memphis, has suggested the use of phenol sulphonate of copper solutions for the colon irrigations.

The writer has used this chemical in the treatment of a number of cases but is unable to state its exact degree of efficiency. It is a very powerful parasiticide and its use is advised alternately with the formalin boric solution. The strength of the copper solution is 8 to 10 grains to each quart of sterile water.

Ichthyol (10\% solution) applied locally to the mucous membrane, or gauze, saturated with the sane solution. packed in the rectum, has seemed to exert a beneficial effect.

It is well to mention here that an antidysenteric serum has been very highly recommended in the treatment of the malignant bacillary type of dysentery.

\section{Chronic or Secondary Amebic Dysentery.}

All sub-acute or chronic cases of dysentery depend for their symptoms upon an ulcerated and inflamed condition which will not yield to treatment.

These cases have exacerbations and amelioration of symptoms. 'They often complain of constipation, which may extend through a period of reeks or even months. It is in these sub-acute and chronic cases that the proctologist is most often consulted.

Such remedies as nitrate of silver, grains 30 to 60 to an ounce of sterile water, or a $20 \%$ solution of argrrol are applied after first cleansing and antisepticizing the rectum and sigmoid with pledgets of cotton wrung out of hot formalin boric solutions. (See Fig. 109.) 
A $30 \%$ solution of lactic acid has also been used to cauterize the nlcerative areas.

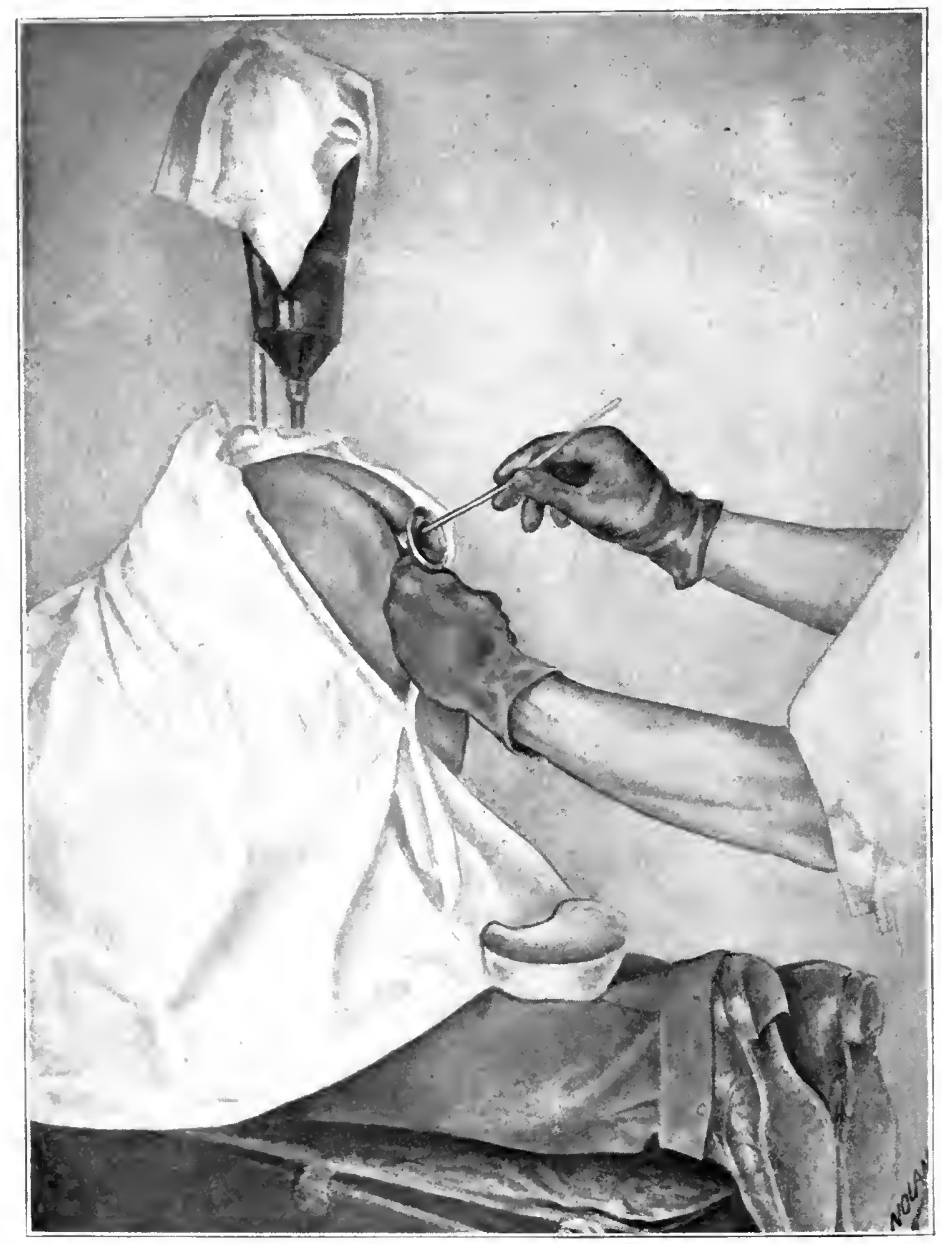

Fig. 109 .

Method of Application of Silver and Other Solutions to the Ulcerated Surfaces of the Rectum and Sigmoid. 
After these applications have been made the bowel is sprayed with some neutral or alkaline solution to neutral-

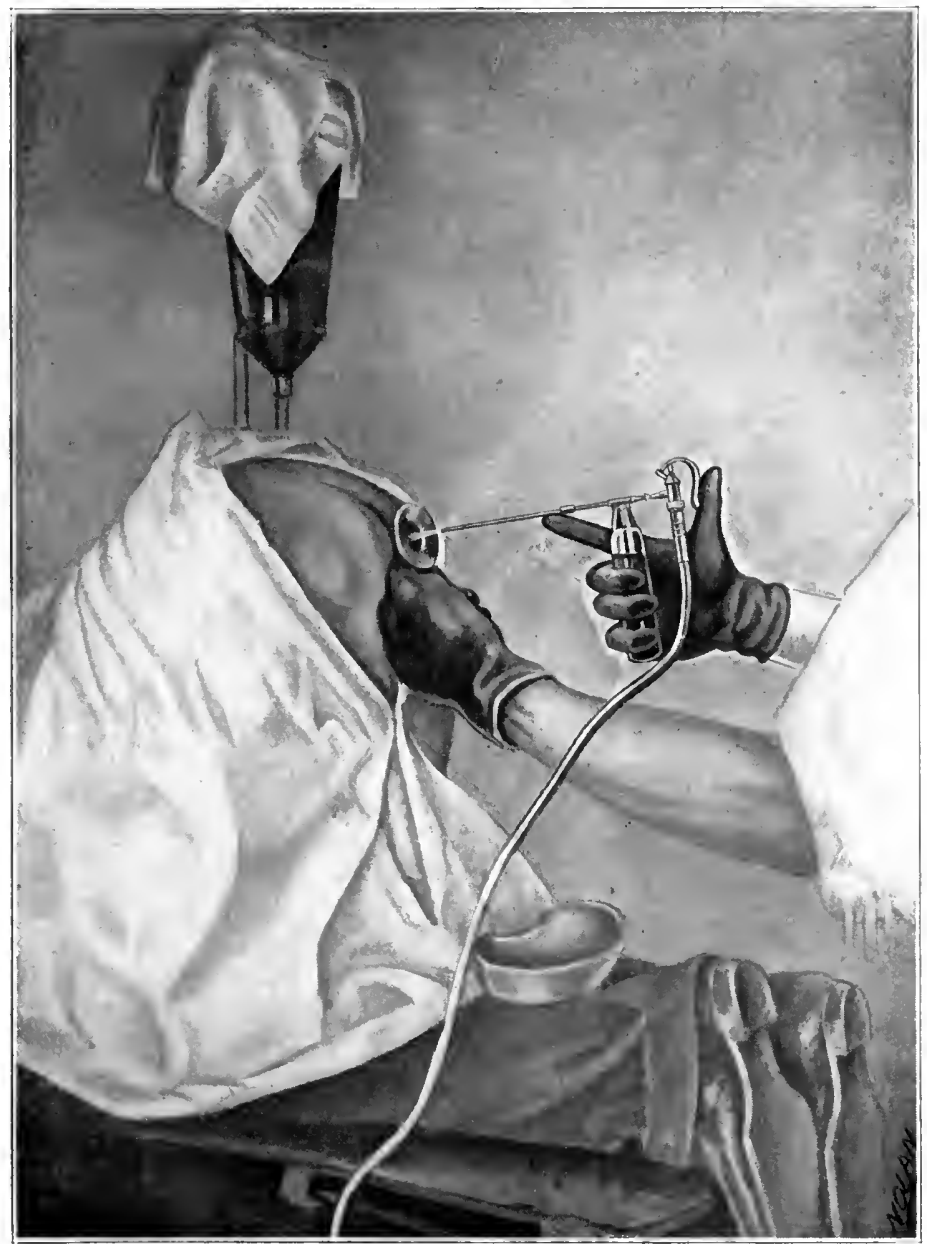

Fig. 110.

Method of Spraying Rectum and Sigmoid, With Solutions and Also Insufflating Mucous Surfaces With Antiseptic Powders. 
ize the excess of the silver or other solution used: (See Fig. 110.)

The bowel surfaces are then dried. Now the insufflation of some non-toxic antiseptic powder such as equal parts of boric acid and aristol is advised.

The symptom of iodism is an unpleasant one and may be readily produced by the instillation of drugs containing iodine into the rectum. Because of this, these remedies, such as aristol, bismuth-formic-iodide and iodoform have appeared most effectual when used just to the point of tolerance.

When the amebic infection has become very chronic, or las extended into all parts of the colon beyond the use of the local measures just described, appendicostomy should be performed and the same irrigations practiced through the appendiceal stump. The water is allowed to pass ont through the rectum into the catch basin.

This plan of treatment was first advised by Dr. E. A. Corsons, of Savannah, Ga.

In 1898 1)r. H. F. Harris stated that some years before 1)1. Corsons had made this suggestion to him. Irrjgations of the bowel with hydrogen peroxide throngh the artificial opening, thus established, were also advised.

About the rear 1901 Dr. Robert Weir, of New York, while performing a colostomy for amebic dysentery, anchored the appendix and irrigated through the stump with a saline solution.

Shortly afterward Dr. Meyer, also of New York, performed a similar operation.

Dr. 'Tuttle, of New York, conceived the plan of allowing the appendix to remain undisturbed after anchorage 
for a sufficient time (three or four days) to establish adhesions about the proximal end before cutting away the distal portion, and using the appendiceal stump lumen through which to irrigate with the desired solutions.

The writer has practised this latter method and irrigated the colon with formalin boric, copper phenol-sulphonate and quinine solutions with most gratifying results. It was observed, however, that the irrigations alone did not effect a cure. Topical applications (per sigmoidoscope or proctoscope) were in all cases used in conjunction.

Dr. J. A. Crisler, of Memphis, in 1906, advised the anchorage of the appendix in a small stab wound below the high incision, which is made with the view of inspecting the liver and gall bladder.

In two chronic cases the writer was forced to perform rectal valvotomies on account of obstruction to drainage, and to the insertion of the proctoscope or even the tube beyond the valves, which were tightly stretched across the lumen of the rectum. This operation will rarely be found necessary.

The writer here wishes to acknowledge with thanks valnable assistance rendered by Dr. H. P. Conley in the preparation of this chapter. 


\section{CHAPTER XIV.}

\section{PROLAPSE OF THE RECTUM IN CHILDREN.}

Prolapsus Recti, or prolapse of the rectum, is the descent, with or without protrusion, of one or all of the coats of the rectum, uncomplicated by any other diseased condition. Prolapsus ani is usually understood to mean the descent and protrusion of either the nucous membrane alone or all of the coats of the anus and lower end of the rectum outside of the anal aperture.

Prolapse may be either partial or complete. Partial prolapse meaning that condition in which the mucous membrane alone protrudes; complete prolapse describing the descent of all of the coats of the rectum. The complete variety is divided into three varieties, according to the degree or extent of the prolapse.

Prolapse of the first degree is the condition in which the prolapsed portion begins at the anal margin, and the mucous membrane covering it can be seen to be continuous with the surrounding skin, there being no sulcus surrounding it. In complete prolapse of the second degree; it will be found that the descent begins at some point in the rectum above the sphincter and is protruded through the anal orifice, being telescoped as it were, through the non-affected portion below. In this variety a distinct 
sulcus can be made out between the prolapse and the margin of the anus.

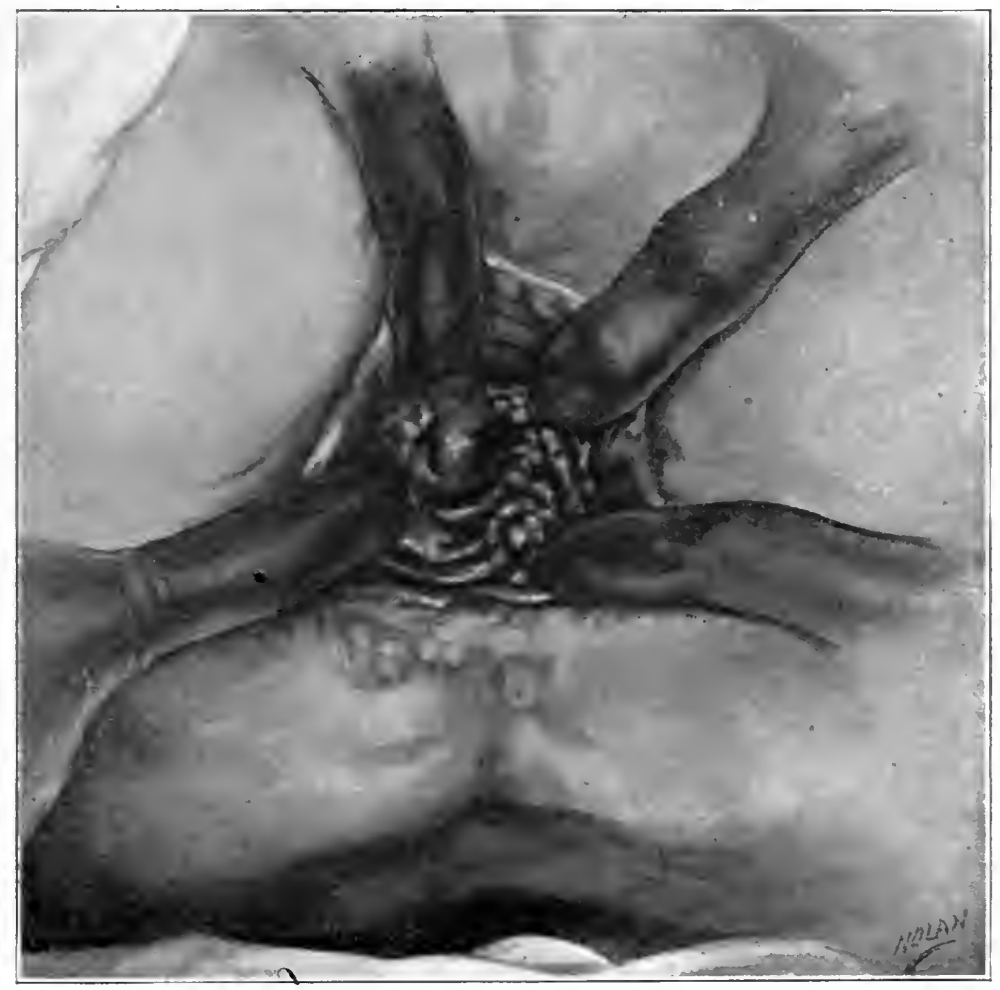

Fig. 111.

Prolapse of the Rectum-Third Degree.

(Made from a photograph of one of the author's cases.)

This shows the prolapsing rectum descending to the anus but not protruding.

Prolapse of the third degree may begin either in the npper portion of the rectum or even in the sigmoid flexure. In this variety the rectum, and even the lower portion of the sigmoid, may descend into the lower rectal cav- 
ity, but as a rule does not protrude from the anus. 'This variety is also known as concealed prolapse. (Fig 111.)

Inasmuch as the limitations of this work does not include those conditions whose relief requires surgical operations under general anesthesia for their relief, none of the conditions mentioned above will be treated, save the condition most commonly seen by the general practitioner-prolapse of the anus and rectum in children. The most frequent variety seen in children is that known as the partial or incomplete, and it consists of an eversion of the anal canal, carrying with it the mucous membrane covering the lower end of the rectum. It is a condition amenable in the vast majority of cases to non-surgical measures, when seen early and treated with patience and persistence.

Etiology. It is brought about most frequently by severe prolonged or undue straining efforts on the part of the child. Such diseased conditions as the presence of a rectal polypus, hemorrhoids, foreign body in the rectum, hard constipated stools, pin worms, stone in the bladder, phimosis, diarrhoea, excessive coughing or sneezing, accompanied by weakness of the sphincter muscle are responsible at times, but most common of all is the prolonged straining efforts at defecation.

The practice so commonly in vogue among mothers in their efforts to train their children to regular habits of defecation has been responsible in the majority of cases for the production of prolapsus recti. The little patient is placed upon the toilet vessel or chair, and is soon made to realize what is expected of him. Sitting in the semi-squatting position which is most conducive to the emptying of the rectum, even of its own mucous 
membrane, for lialf an hour ; or even all the morning (as has happened in some cases which have come under the author's notice), the little one using all his efforts in order to accomplish his daily duty, gradually brings about a separation of the mucous membrane of the rectum, with accompanying protrusion from the anus.

In other cases, througl extraordinary efforts of the abdominal muscles, the mesentery of the sigmoid becomes elongated and an intussusception of the upper rectum and lower sigmoid takes place. Protrusion of the prolapsed bowel is very rare in this instance, and a condition known as concealed prolapse is produced and often goes undiagnosed for a considerable period of time. From an anatomical point of view, the straightness of the sacrum in children offers less support to the rectum than in adults, and in children who have been suffering from wasting diseases, the parts become so relaxed that practically all support is taken away from the rectum.

Symptoms. When the rectum prolapses in children, it appears rather unexpectedly. After a more or less long period of time, in which the "training", of the child has been going on, the mother is surprised, some fine day, by the appearance of a ring of red or purple hued membrane surrounding the anus, the size depending upon the amount of rectum prolapsed. The longer the prolapse remains ontside the rectum, the more purple hued it becomes from the interference with the return circulation on account of the contraction of the sphincter.

Diagnosis. The diagnosis is very simple; in fact, selfevident. The appearance of a ring of soft, velvety mucous membrane protruding from the anus, is indicative only 
of one condition, that of prolapse. A polypus would be differentiated by its rounded form, harder consistency and the presence of a pedicle belind the protrusion extending into the anus. Hemorrhoids, which are rare in children, would be gradual in onset; would be of firmer consisteney, forming separate masses, and would not exhibit the peculiar red or purplish appearance of prolapsed mucous membrane. On each succeeding occasion, when the bowel is protruded, more of the mucous membrane comes down, and in aggravated cases the entire rectum may be protruded.

Treatment. When the protrusion first makes its appearance it may be reduced in the following manner: The child is placed on its mother's lap with the buttocks raised considerably higher than the head. A compress soaked in ice water placed against the prolapse will often be all that is necessary. Gentle pressure will in a few minutes, in most cases, cause a return of the prolapsed portion. Oftentimes simple digital pressure of one side of the prolapse while the buttocks are separated with one hand, and steady pressure made with the fingers of the other, will suffice. The other half is then treated in like manner.

Where the prolapse has remained outside long enough to become swollen, oedematous or congested, and the sphincter has contracted upon it; it will often be very difficult to return the prolapse unless the sphineter has been relaxed by the injection of a local anesthetic. In order to relieve the congestion and shrink the blood vessels, the employment of compresses, soaked with one to one thousand solution of adrenalin chloride and applied 
with firm pressure to the protrusion has in the author's hands, been found extremely satisfactory. 'The blood vessels become constringed and the mass much reduced in size, and reduction is comparatively easy.

Whenever pressure is used in this region, it should be firm but gentle, as it would be very easy to do serious damage if the manipulation were rough or violent. Wrapping dry absorbent cotton around the index finger and pressing firmly against the prolapse and in the direction of the rectal canal, will often return a prolapse with ease. The finger is withdrawn in a twisting manner so as to allow the cotton to remain in the rectum, from whence it is passed with the next stool.

If the child's habits are corrected, the bowel, in many cases, will not protrude again. In cases, however, where the protrusion recurs, a definite line of treatment must be undertaken in order to relieve the tendency to chronicity of the condition. Any exciting cause, such as stone in the bladder, phimosis, pin worms, polypus, foreign body in the rectum, etc., must be relieved by proper surgical measures. If the case is due to constipation, the child's dietary should be looked into and corrected.

Where the case is one, however, where the prolapse has been brought upon by the prolonged sitting at stool, with its coincident severe straining efforts, this method of training must be dispensed with. The child must be made to move its bowels in the recumbent position, either lying on its back or side, preferably the latter. It must not be allowed to have movements in the sitting posture while under treatment. The administration of white petroleum oil or liquid albolene, suitably flavored, in doses 
varying frour ten minims four times a day in an infant, to a teaspoonful for the child of five or six years of age, should be resorted to in order to keep the stools soft and the intestinal canal well lubricated. It is important after the bowel movements to strap the buttocks together with strips of adhesive plaster; and in some cases it may be advisable to keep a pad made of absorbent cotton, wrapped with gauze, firmly against the anus.

This treatment will be very successful if persisted in long enongh. The author would advise two months as the average length of treatment in the average case. Any tendency towards diarrhoea should be immediately looked after, and the dietary canse for it discovered and corrected, for the violent peristalsis which accompanies diarlioea is often productive of as bad, if not worse, results, than the straining efforts of constipation.

Concealed Prolapse.-In some cases of constipation, so-called, in infants, all efforts for successful treatment will fail; and the author would advise in these cases the examination of the infant's rectum by means of a smallsized proctoscope or a large female cystoscope. Occasionally, this method of examination will be rewarded by the discovery of a prolapse of the third degree (Fig. 111), which extends down to the rectum but does not protrude. In these cases the infant will be very fussy and will strain until red in the face, but all that rewards his efforts will bc a small quantity of mucus stained with fecal matter; and the only way in which the child's bowels can be emptied is by means of enemas. The same treatment as ontlined for the incomplete prolapse is indicated in this condition. 
The principal point in the prevention and the treat. ment of prolapse of the rectum in children, is the education of the mothers along the line of the so-called training of infants. While it is not the province of this work to go into the subject of infaint feeding, nevertheless, the anthor feels that if more attention is paid to the presence of sufficient hydrocarbon elements in the child's dietary, and the child is made to drink sufficient water, much good would result. Instead of forcing the little one to sit upon the toilet seat from half an hour to an hour and a half, or even longer, the child's bowels would move with regularity and with ease and prolapse would become a very rare condition. The squatting posture as assumed by the aborigines is the best for the children to assime. If after ten or fifteen minutes at the stool the child does not have a morement, it is better far to insert a soap suppository or administer a small enema to tide it over occasionally, than to indulge in the pernicious custom, seemingly so prevalent.

When in spite of strapping and the proper control of the bowel movements, the prolapse still persists in reappearing, it becomes necessary to do something more radical. The method which has been most satisfactory in the hands of the author and which is particularly adaptable to prolapse of the rectum in children, is what is known as linear cauterization. This may be accomplished in two ways-either by application of strong nitric acid or the use of the actual cantery: Neither method is applicable with entire satisfaction unless a general anesthetic is employed. Nitrous oxide, however, can be used in these cases with perfect safety and makes a very dependable and satisfactory anesthetic. 
Canterization by Nitric Acid.-The child is placed in the lithotomy position with the prolapse unreduced, and is placed under the influence of the nitrous oxide gas. The protruding mucous membrane is wiped dry and a

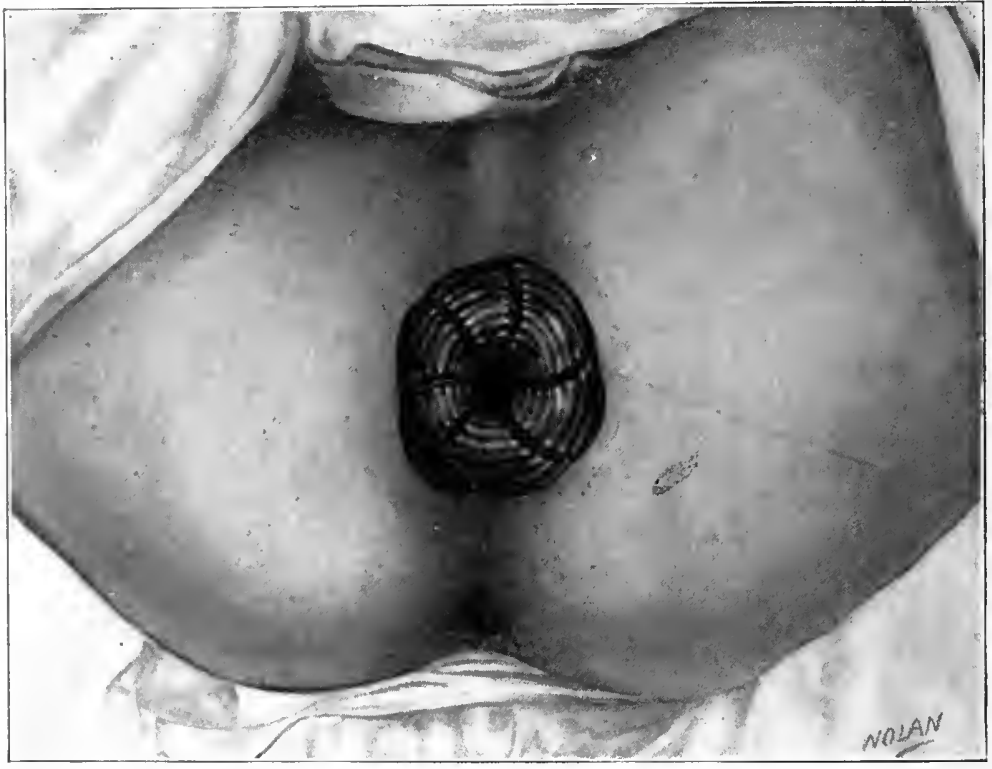

Fig. 112.

Prolapsus of the Rectum, First Degree, Showing Radiating Lines of Cauterization.

wooden applicator, one end of which has been wrapped with a very small quantity of absorbent cotton moistened with fuming nitric acid, is all that is necessary. The acid is applied in 4 to 6 radiating lines (Fig. 112), beginning at the uppermost portion of the center of the prolapsed mucous nembrane at the lumen of the gut, and with considerable pressure a line is drawn or painted to, but not touching, the muco-cutaneous juncture. Four to six 
equidistant cauterizations are made in this manner and an ointment composed of a drachm of bicarbonate of soda to an ounce of petrolatum freely applied. A piece of rubber drainage tube the size of a lead pencil, is wrapper with gauze until it forms a plug or packing abont $3 / 4$ of an inch in diameter in its center and tapering at its extremities, is used to force the prolapse back into the rectum, and is left there for three or four days if possible. The little patient's suffering after the operation is not very acute, but if there should be much pain, it should be controlled by suitable doses of codein or: morphine hypodermatically; $1 / 8$ to $1 / 4$ grains of codein will answer very nicely in children from $1 \frac{1}{2}$ to 3 years old.

The after treatment consists in the same methods and procedures as that advocated above in regard to diet, defecation in the recumbent position, the strapping of the buttocks, etc. After three weeks the child may be allowed to resume defecations in the squatting position. In the first dressing immediately after the operation it is wise to exert some pressure against the anus, by means of a suitable pad kept in place by adhesive plaster straps.

Linear Cauterization with the Actual Cautery.-The patient is prepared as described in the preceding paragraph and when the prolapse is protruding to its fullest extent, a Paquelin cautery armed with a blunt point, and heated to a white heat is used for making the cauterization in the same manner as the nitric acid is used. (Fig. 112.) One should be careful to carry the cauterization through the mucous membrane and into the muscular layer, but should be extremely cautious about burning through the muscular tissue. The amount of destruction 
of tissme is more apparent than real; one must remember the object of the cauterization is to accomplish the contraction of redundant tissnes, and it is the contracting scar which invariably follows the use of the cantery, upon which we depend to accomplish the results. In this condition we take advantage of the great contraindication to the use of the actual cautery in the surgery of the rectum, for we well know that the scar produced by a burn on mucous membrane, invariably contracts to such an extent as to lessen the calibre of the rectum. The after treatment dressing, packing, is the same as described where nitric acid is used as a cauterizing agent.

Where these methods fail, there is nothing left to do but one of the cutting operations under surgical anesthesia and preferable in hospital surroundings. When such is the case, the operation had best be done by one who is specially trained in this line of work, and not by the general practitioner; as the operative and after-care often taxes the patience, skill and ingenuity of even the trained specialist to accomplish the desired results. 


\section{CHAPTER XV.}

\section{THE TECHNIQUE OF THE USE OF LOCAL ANES- THESIA IN THE TREATMENT OF ANO- RECTAL DISEASES.}

If any excuse or apology were necessary for the presentation of this work to the profession at this time, the subject matter contained in this chapter will be ample justification. The dangers, inconvenience, and necessary confinement to bed, and detention from business, which must attend the use of general anesthesia in many socalled minor operations, has created a demand and constantly enlarging field for the use in many departments of surgery, of local anesthetics. In the surgical treatment of diseases of the rectum and anus this is especially true; and while there are many diseased conditions of this region requiring surgical interference, the extent of which makes their operative treatment impossible without general anesthesia; there are, nevertheless, many of the more common diseases of this part of the body which are entirely amenable to surgical treatment under regional anesthesia.

The development of the nse of local anesthesia in the treatment of anal and rectal diseases has progressed to such a stage, that it is safe to say, that fully $75 \%$ of 
all cases of rectal and anal disease are amenable to treatment without the use of general anesthetics.

Various anesthetic agents have been employed for the production of local anesthesia in this region, among which may be named the ethyl chloride spray, and the injection of solutions containing cocain hydrochloride, beta eucain hydrochlorate and lactate, alypin, stovain, novocain, chloretone, as well as plain sterilized water.

Formerly, cocain in solutions varying in strength from 4 to 10 per cent were used. Symptoms of an alarming nature frequently developed after the injection of but a few drops of even a 4 per cent solution, which clearly demonstrated the toxic properties of the drug and the dangers of its indiscriminate use in strong' solutions. 'Today we know that the extent of anesthesia produced depends, not so much on the strength of the solution, as upon the pressure anesthesia produced on the nerve endings, by the amount of solution injected, rather than its strength.

Today, therefore, practitioners who are still partial to cocain are using solutions for injection, varying in strengtl from 1-10 of one per cent up to one-half of one per cent, and find the latter strength equal to the severest test. The author, after a trial of all of the anesthetics mentioned above, places his main reliance on beta eucain lactate where he requires a chemical anesthetic; and plain sterile water in some few selected cases. The lactate of beta encain is used in preference to the hydrochlorate because of the fact that solutions of the former salt can be sterilized by boiling without detriment.

'The strength of the solution varies according to the part to be anesthetized, as well as the amount of work 
to be done. For injection into the skin, and for the anesthetization of the sphincterian nerves, one-fourth to onehalf of one per cent solution is strong enough. For the distension of the tissues, for instance, in operating for fissure or internal hemorrhoids, a one-tenth of one per cent solution will suffice. Another important reason for my preference for eucain over cocain, is the fact that eucain is less than one-half as toxic as cocain, and is fully as powerful in its anesthetic properties. Chloretone, in the strength of one-half of one per cent, may be used with impunity in place of the one-tenth of one per cent solution of encain in such operations as require considerable amount of solution. It is not toxic and has the added advantage over the other drugs of being an antiseptic as well as an anesthetic. The use of sterile water as an anesthetic in the treatment of rectal and anal diseases, was exploited prominently a few years ago, and while the author's experience with it has proven to him that satisfactory anesthesia in certain cases can be produced by its use alone; he limits its use in his work at present to the distension of internal hemorrhoids only. The one objection which he has found to its indiscriminate use is the larger degree of discomfort to the patient at the initial

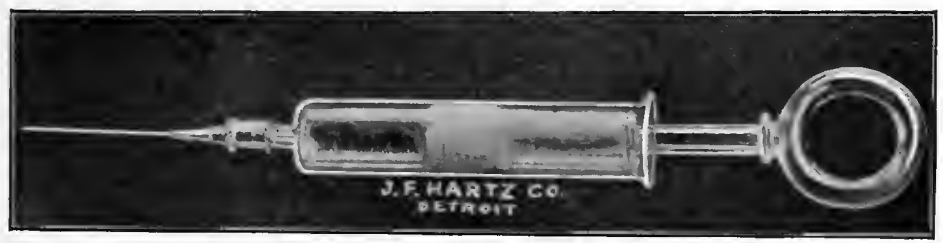

Fig. 113.

Aseptic All-Glass Hypodermic Syringe Provided With Asbestos Packed Plunger. 
injection, and the large quantities of solution required in some operations in the sphincterian region, cansing such distortion of the tissues, as to not only impede the work of the operator; but to displace the parts so that accurate work could not be done.

It is well for the reader to realize that in "a pinch" sterile water can be used in lieu of any chemical anesthetic, and there are occasions when he may be called upon to do work in an emergency, where the varions chemical anesthetics may not be available; when, with an ordinary hypodermic syringe and boiled water satisfactory anesthesia can be produced.

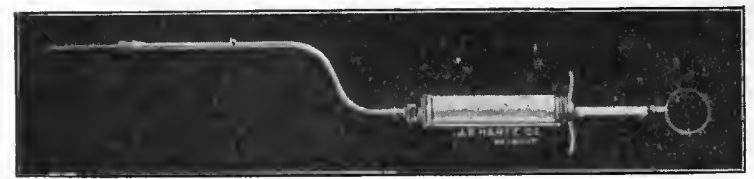

Fig. 114.

Aseptic All-Metal Syringe Provided With Extension for Infiltrating Through the Proctoscope.

The principal instruments required for the production of local anesthesia is a hypodermic syringe such as is used for the injection of antitoxin; which may be constructed either entirely of metal or glass (Figs. 113 and 114) so that it can be readily sterilized by boiling. This syringe should have a capacity of from 2 to 4 drachms. The needles used should be the finest that can be procured and the points should always be kept sharp. A quick puncture with a sharp pointed fine needle is almost painless, while the use of a larger calibred needle with a short beveled point, will cause considerable and umnecessary discomfort to the patient. The piston syringe package, con- 
structed of glass and rubber, which many of the manufacturers of antitoxin supply, when sterilized by boiling, makes a fairly good substitute for the regular aseptic hypodermic syringe, and in the absence of the proper apparatus it may be used. The objection to it is the fact that the needles supplied with it are usually of large calibre and not as sharp as they should be for this work. The only other piece of apparatus required (and even that is not an absolute necessity) is a portable mechanical vibrator, armed with a cone-shaped rectal vibratode, (Fig. 117), for use in the dilatation of the sphincter muscle.

The solution used should be accurately prepared as to the percentage of chemical anestletic used. Where beta eucain lactate is employed, the solution is made up and placed in an ordinary test tube. It is sterilized by boiling over the flame of a Bunsen burner or spirit lamp, and then stoppered with absorbent cotton and allowed to cool. The solution is prepared freshly for each operation.

The patient is prepared for the operation as follows: Twenty-four hours before the operation, he is given a brisk cathartic and is instructed to partake of nothing but liquid food thereafter. On the morning of the operation the bowels are washed out by means of a large soap enema, and he is directed to report at the office about onehalf hour before the time for operation. He is then given a quarter of a grain of morphine by mouth.

When ready to operate, the patient is placed upon the table in the left lateral position, the left leg being extended and the right well flexed. The clothing is placed well out of the way, and the patient covered with clean 
sheets. The anus and perineum is shaved and scrubbed with liquid antiseptic soap, then washed with a one to 1000 solution of iodide of mercury, which is washed off

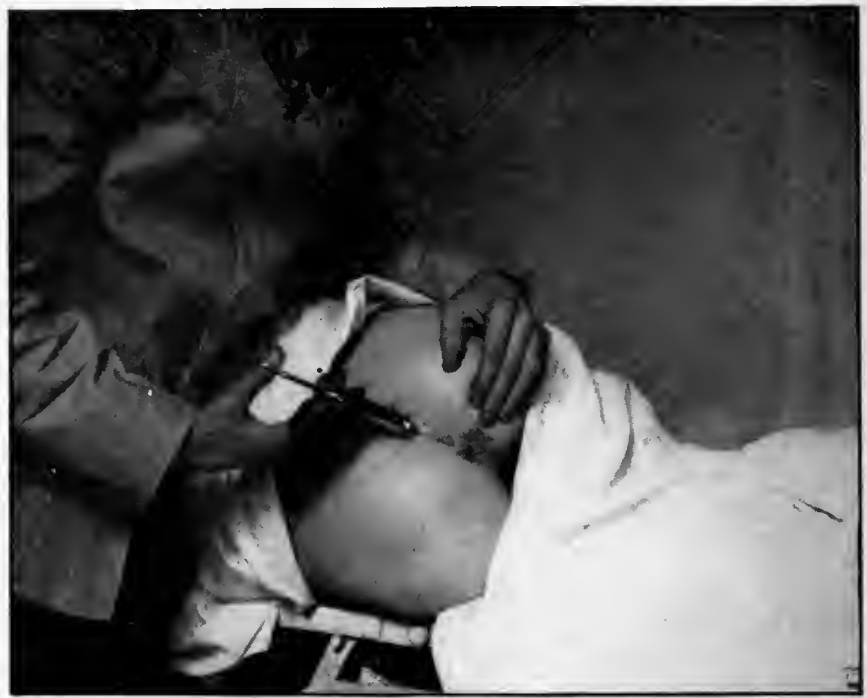

Fig. 115.

Exact Point of Puncture for the Injection of Local Anesthetics for Dilating the External Sphincter.

With the patient in the lateral position, a point from $1 / 4$ to $1 / 2$ inch posterior to the posterior commissure of the anus is chosen for the first injection.

with sterile water, and a compress of alcohol applied. A point one-half inch below and posterior to the posterior commissure of the anus is selected. (Fig. 115.) A spray of ethyl chloride or the application of a drop of pure carbolic acid is used to lessen the pain which accompanies the introduction of the needle. Wherever it is possible, the index finger of one hand, protected by a finger cot and well lubricated, is inserted in the anus; and the sphincter is pulled downward and backward. The syringe 
containing about one drachm of one-half of one per cent solution of eucain lactate, with a fine sharp-pointed needle about two inches in length attached, is held in the other hand. 'The needle is inserted quickly, just underneatlı the

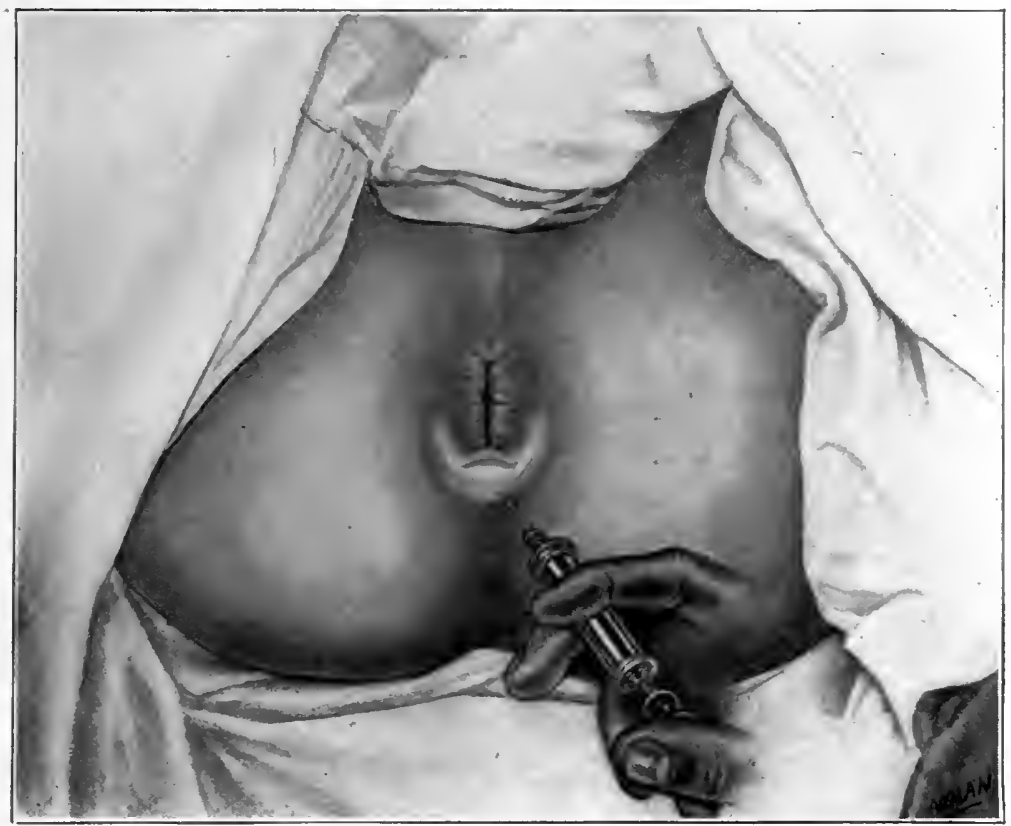

Fig. 116.

Showing the Amount of Distension of the Tissues Necessary in Anesthetizing the Sphincters.

skin, and 4 to 5 drops of the solution slowly injected. One should be extremely careful about injecting the solution too quickly, as this part of the procedure is the most painful and often needlessly causes suffering, particularly to the timid and neurotic patient. The point of the needle is then passed inward and laterally, going down towards and into the external sphincter muscle which 
guided by the finger in the rectum is brought down towards the needle. The point of the needle should be kept about one-lialf inch from the anal aperture and the injection is carried up along the posterior lateral quadrant of the anus for about three-fourths to an inch. The needle is then retracted to the point of puncture but not withdrawn. It is then pushed up on the other side in the same mammer, injecting the opposite side; so that when the injection is completed the wheal of infiltration is U-shaped, the apex being at the point of puncture. (Fig. 116.)

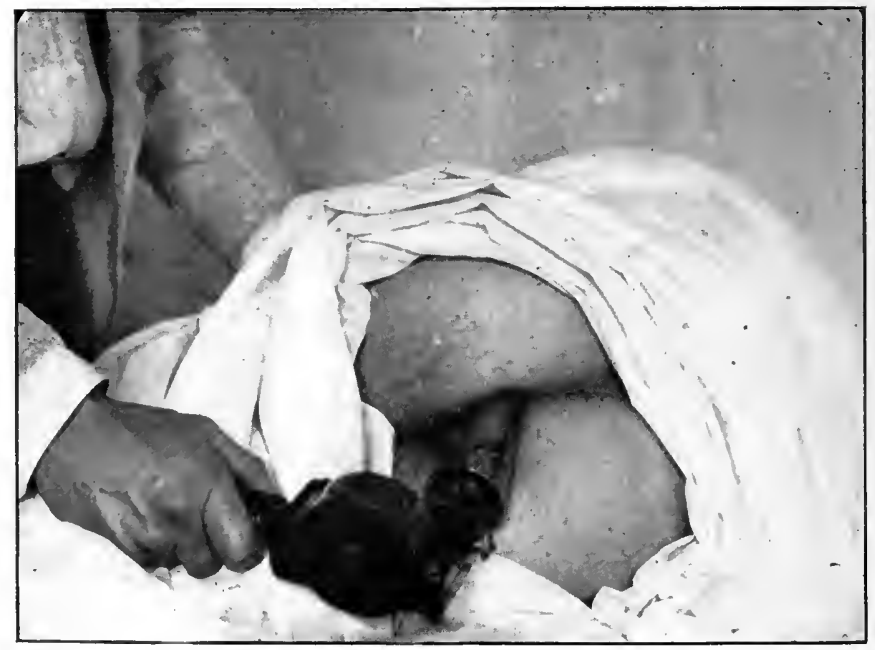

Fig. 117.

Pcsture and Method cf Producing Dilatation of the Sphincter Ani by the Use of a Pcrtable Vibrator Armed with a ConeShaped Vibratode.

This technique allows of the anesthetization of the sphincterian nerves of both sides from but a single puncture. Care should be taken lest the rectal wall be punc- 
tured, but with the index finger of one hand in the anus during this procedure, such an accident should not occur. 'Three or four minutes are allowed to elapse to allow complete anesthesia to take effect; then the vibrator, to which has been attached the cone-shaped vibratode, well lubricated, is pressed against the anus. (Fig. 117.) With very little pressure, but with the apex of the vibratode kept in the direction of the axis of the ano-rectal canal ; from two to three minutes vibration will dilate the sphincter painlessly to a sufficient calibre to allow what.

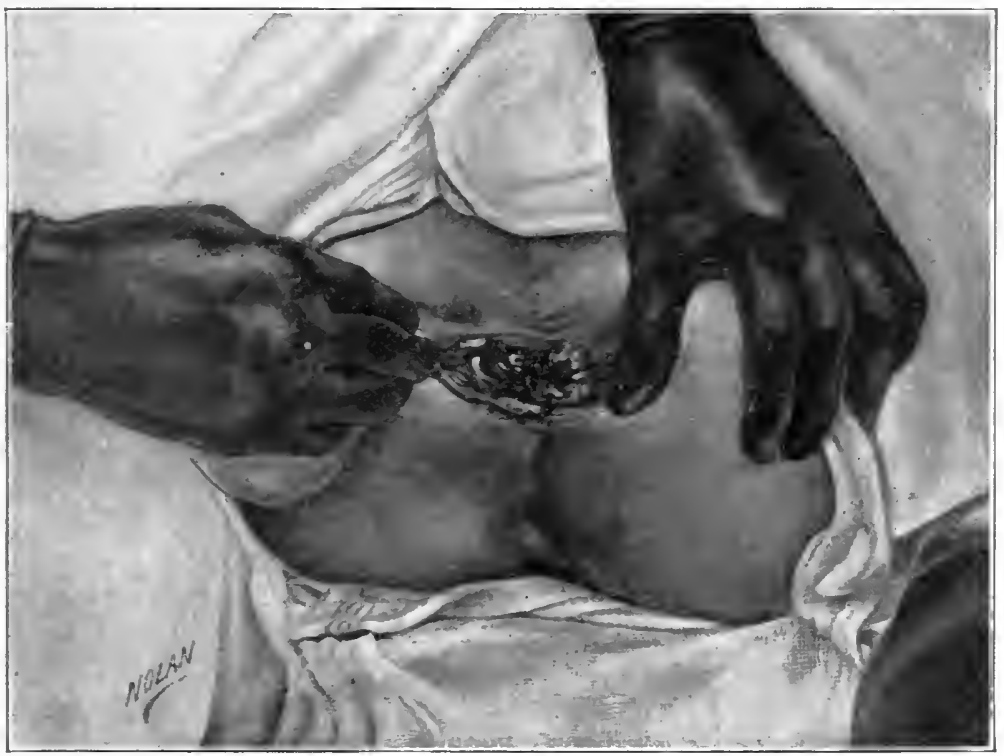

Fig. 118 .

The Amount of Dilatation of the Sphincter Under Local Anesthesia.

This drawing made from a photograph of one of the author's cases of internal hemorrhoids, well illustrates the amount of dilatation of the sphincter, which may be produced under locil anesthesia. While complete divulsion is neither possible nor necessary, sufficient distension is here secured to successfully remove the internal hemorrhoids seen in the drawing. 
ever operation is to be done, to be accomplished without difficulty. (Fig. 118.) Complete divulsion of the sphineter can very rarely be accomplished by this means, but the dilatation will be amply sufficient for our purposes.

The vibrator is a very convenient apparatus to have at hand, as the dilatation can be more quickly and evenly accomplished by its use. In its absence, however, one may use the index fingers of both lands protected by finger cots or rubber gloves, and by a gentle to and fro massaging movement, gradually accomplish dilatation in a very satisfactory manner. One should never use any of the dilating rectal speculums in the dilatation of the sphincter. The fingers are far better dilators, and can do no damage with intelligence and care behind them to guide them.

The technique for operating for the various conditions amenable to operative treatment under local anesthesia will be dwelt upon more in detail in their respective chapters, while the differences in technique of anesthetization will be taken up below. Suffice it to say, however, at this point, that no operation upon the anus or rectum should be undertaken under local anesthesia, which will require extensive dissection or over twenty minutes of time for its completion.

External Hemorrhoids. If the hemorrhoid is entirely external and is not complicated by any other anal condition, it will not be necessary to anesthetize the sphincter. After the usual preparation for operation, the most dependent hemorrhoid is injected from its base with one-half of one per cent solution of eucain lactate, about 20 to 30 minims being used directly under the skin. If 
ANESTHETIZATION FOR THROMBOTIC HEMORRHOIDS. 305

further distension is required in order to produce complete anesthesia, sterile water may be used for the deeper injection. After two minutes the skin may be incised painlessly and the operation proceeded with. Where more than one hemorrhoid is to be operated, they may all be anestletized at once, if the operator is rapid in his work; otherwise they had best be anesthetized separately when ready to operate on each.

Acute Thrombotic Hemmorhoids. The acute thrombotic lemorrhoid is usually single, occurring just at the anal margin. After being prepared for operation, eight or ten drops of the one-half per cent eucain solution is injected just beneath its outer covering, whether skin or mucous membrane; care being taken not to inject deeply and into the clot. Sufficient solution should be used to distend the tissues over the clot and blanch them to whiteness. It may then be incised painlessly and the clot turned out. It is well after the turning out of the clot to inject the tissues beneath it, and examine carefully as sometimes a second clot may be found beneath the first, which must be removed in like manner.

Perianal Abscess. In those cases of perianal abscess not extensive enough to require general anesthesia for their operative treatment, the use of a local anesthetic is well adapted. The technique of injection is the same as that outlined above for thrombotic hemorrhoids. The reader is cautioned to make his injection very carefully, so as not to perforate the abscess cavity with the needle. The solution must be injected into the skin itself and directly under it. After waiting two or three minutes for anesthesia to take place, the abscess may be opened with absolutely no pain. 
Fissure in Ano. In all cases of fissure, the sphincter should be anesthetized and dilated. In many cases where the fissure is situated low down, the anesthetic solution injected for the anesthetization of the sphineter, will also be sufficient for the incision or excision of the fissure as well. Where the fissure is more extensive and with an indurated base, or is located at some other portion of the anns than its usual site, the posterior commissure; it must be injected separately. One-tenth of one per cent solution of eucain or one-lualf per cent solution of chloretone may be used. The stringe should be filled. The needle should be inserted about one-quarter of an inch below the outermost extremity of the fissure, or beyond the sentinel pile when one is also present.

The skin and mueous membrane surrounding the fissure or induration, as the case may be, should be infiltrated to such an extent, that the fissure is raised on a white waxy looking mound, and lies, as it were, on a water bed. It may require as much as three drachms of solution, but distension of the tissues is essential before thorongh work can be done. Anesthesia should be carried below the base of the fissure for at least a quarter of an inch.

Fistula. The only variety of fistula in which it is advisable to use local anesthesia as a routine measure is that of a simple, shallow, complete, fistula whose course is direct and not branching. A blind external or internal fistula whose opening is not over one inch from the anus and whose extent can be accurately gauged, may be opened under local anesthesia. As a general proposition, with the exception of the three. 
varieties mentioned, general anesthesia (nitrous oxide wherever possible) should be used in operations for fistuia in ano. The sphincter should be anesthetized in all cases. The skin and mucous membrane above the fistula should be infiltrated with the 1-10 per cent eucain solution and then by successive injections the entile fistulous tract surrounding with the injected anestletic fluid. The infiltration should be carried to the point of blanching. The operation then may be proceeded with as outlined in the chapter on fistula.

Hypertrophied Anal Papillae. In cases where liypertrophy of the anal papillae is not accompanied by a tightly contracted sphincter, it is possible to remove the papilla under local anesthesia without dilatation of the sphincter. It is advisable, however, in order to overcome the tenesmus and painful spasmodic contractions of the sphincter following any operation in the anal canal, to anesthetize the sphincter as a general rule in removing these papillae. There this is done the anus is held open by means of a retractor and each papilla is injected from base to apex with the 1-10 of one per cent eucain solution. It may then be removed painlessly, and each successive one injected in turn before removal.

Where the sphincter is not anesthetized, the use of a short anoscope such as has been described by the author, with its internal opening on the slant, will be required. The papilla, as it hangs down or projects into the opening of the anoscope, is injected by means of a long needle attached to the hypodermic syringe, and injected as described above. Where it is desired to open the crypts of Morgagni as well, the needle should be carried up for 
half an inch or so, when, after the removal of the papilla, the crypt can be split open at will.

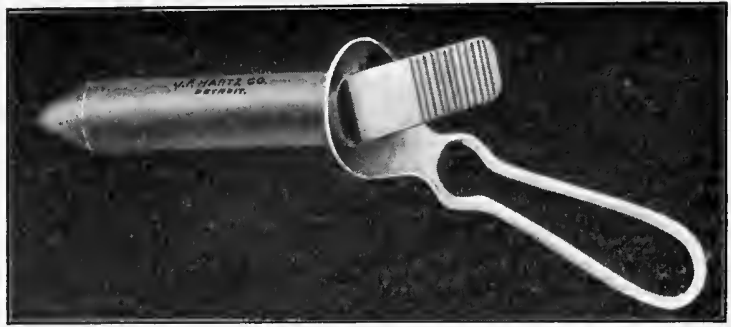

Fig. 119.

Author's Modification of the Martin Operating Proctoscope.

The obturator has a conical shaped extremity and is made of metal.

Hypertrophied Rectal Valves. In operating for the section of hypertrophied Houston's valves, the dilatation of the sphincter as outlined above, is often the only part of the operation, where a local anesthetic is required. The valves themselves are very poorly supplied with sensory nerves, and as a result, incision is painless. In some cases, however, there is some sensitiveness to pain; so it is wise in all cases to be on the safe side, and apply by means of an applicator bent at a right angle, a four per cent solution of beta eucain to both upper and lower surfaces of the valve. After waiting two minutes, opera. tion may be begun.

Removal of Foreign Bodies. Oftentimes small splinters of bone, pins or other swallowed foreign bodies will traverse the entire gastro-intestinal tract without doing any injury, or becoming lodged, until they reach the lower end of the rectum, when they impinge against the rectal aspect of the mucous membrane covering the sphincter muscle. By their constant irritation, they cause 
spasm of the muscle and intense suffering. On account of the tonic contraction of the sphincter, which is caused by this irritation, any attempt at the insertion of a proctoscope or even the finger is usually futile. The dilatation of the sphincter by means of the technique outlined above, is nowhere more applicable than in this class of cases, and not only such foreign bodies as have been mentioned, but fecal concretions and impactions of considerable size can be removed without the employment of a general anesthetic as well.

Removal of Benign Perianal Growths. Small benign growths situated at or near the anal orifice, such as dermoids, sebaceous eysts, lipomata or condylomata are very satisfactorily removed under local anesthesia, under the following technique:

After the parts are cleansed, sterilized and shaved, condylomata are removed by the application of a 4 per: cent solution of eucain to the parts, which is repeated every two or three minutes for 10 minutes. Then if anesthesia is not complete, the parts are sprayed with ethyl chloride solution, the condylomata quickly snipped off with sharp scissors curved on the flat, and fuming nitric acid applied witl a wooden applicator, or a small tight swab. Boro-chloretone powder is then applied, and the parts covered with a gauze dressing. In the case of a dermoid or sebaceous cyst, or fatty tumor, the technique is the same for the removal of any of the three varieties. The skin covering the tumor is first injected with one-half per cent solution of eucain lactate, a wheal or welt being formed over the proposed line of incision. The incision is made and the tissues above and surrounding 
the tumor infiltrated with one-tenth of one per cent solution of encain lactate or sterile water; when the dissection and removal of the growth can be accomplished easily, with forceps and scissors. Care should be taken in the cases of a cystic tumor not to puncture the cyst wall with the injecting needle, and in the excision of the growth to be sure to remove all of the sac. If this is not done, recurrence is liable to follow.

Posterior Internal Proctotomy for annular stricture situated in the anal canal, or not over one-half an inch above the ano-rectal juncture.

With the patient in the left lateral position, and prepared for operation, the region posterior to the anus, anal canal, and stricture, is infiltrated with one-tenth of one per cent solution of eucain lactate. After waiting two or three minutes for anesthesia to take full effect, the stricture is divided in the posterior median line down to the rectal wall, with a sharp scalpel, a piece of gaume inserted, and the operation is complete. The author's technique for rectal valvotomy by the use of the rubber ligature may be substituted for the incision, if the calibre of the stricture is sufficiently large enough to admit the ligature carrier. After operation, the recurrence of the stricture is prevented by the introduction of Wales' bongies up to size No. 12, twice a week at first, and at increasing intervals until complete healing has taken place.

After carefully perusing what has been said regarding the employment of local anesthesia, and bearing in mind the contra-indications and objections as outlined in the following chapter on Limitations of Local Anesthesia; 
other diseased conditions of not only the rectum and anus, but in other parts of the body, will present themselves, in which the employment of local anesthesia will be found very advantageous; and the results obtained therefrom fully as successful as where heretofore, the employment of general anesthesia has been thought absolutely necessary and indispensable. 


\section{CHAPTER XVI.}

\section{THE LIMITATIONS OF OFFICE TREATMENT AND INDICATIONS FOR OTHER MEASURES.}

While the primary object of this work has been to bring before the profession, the advantages to be gained from the treatment of various rectal diseases in office practice, and to demonstrate the advantages of the use of local anesthesia in the treatment of many of the more common conditions met with in connection with the treatment of diseases of the anus and rectum; it has been thought wise to utter a warning note, lest the reader be led away by over-enthusiasm.

While the author believes that the field for the employment of local anesthesia in rectal surgery, as well as in other branches of practice, is rapidly widening; he wishes to impress upon the reader that this field has definite limitations and that there is, and always will be, a large class of cases whose successful treatment requires more radical measures, which only can be employed by the aid of full surgical general anesthesia.

If the reader has carefully read what has been said upon means and methods of diagnosis, and has noted in the various chapters following, the class of cases in which the author advocates the use of non-surgical meas- 
ure and the employment of local anesthesia; he will have noted that the methods of treatment advocated are confined to a very definite class of cases. All of the conditions treated of, have been located either at, or in the immediate vicinity of the anal canal, or were those affections of the mucous membrane of the rectum or lower sigmoid, which are accessible to treatment through the proctoscope or sigmoidoscope.

The first thing one should remember before commencing the treatment of any pathological condition found in the region of the anus, is that until a careful exploration of the entire rectal cavity has been made, and every portion of it examined with the eye; he has not made a diagnosis, and has no right to treat the patient until he has. It would be a sad and unfortunate discovery for the physician who has been treating an anal ulcer, or pruritus, or hemorrhoids, to discover after several weeks, that the condition under treatment was merely secondary to an extensive ulceration higher up in the rectum, a stricture, or malignant disease.

In women, suffering from pelvic troubles which may require laparotomy for their relief, the removal of any minor rectal condition present under local anesthesia, had better be postponed, and the rectal or anal condition treated at the time of laparotomy.

In patients suffering from irregularity or interruption of their normal bowel movements, it is wise to exclude by careful abdominal examination any possibility of chronic intestinal obstruction, due to some abdominal growth, displacement, or adhesions; than to attempt to relieve the patient by means of rectal dilatation and massage. 
Every patient presenting himself with a fissure or ulcerative condition of the anal canal, should be carefully questioned as to the possible history of previous syphilitic infection. The presence of gonorrhoeal discharge, is a contra-indication to operative measures until the disease is remedied. In women, a purulent vaginal discharge as well as the menstrual flow is of course, a contra-indication.

Patients suffering from anemia are always bad subjects for operation at one's office under local anesthesia, and a history of hemophilia should always be excluded before office operations. Patients of a highly neurotic temperament and hysterical females are best operated at home or in the hospital, and under general anesthesia. In other words, the suitable cases for office treatment are those suffering from diseased conditions, whose pathological source is located either on the mucous surface of the rectum and lower sigmoid, and is definitely circumscribed in area and not of a malignant, syphilitic or tubercular type; or to lesions occurring at or around the anal orifice, whose outlines can be definitely marked out by the diagnostic means outlined in the fore part of the book.

One of the greatest satisfactions to the practitioner who as a routine measure makes a proper rectal exami. nation of his patients whose srmptoms would seem to indicate it, is the discovery of commencing malignant disease early enough to allow of the removal of the primary focus, and the saving of his patient's life. As has been said before, a history of rectal hemorrliage, however slight, is an imperative demand for complete explo- 
ration of the rectal cavity and the most important condition to be on the lookont for, which makes itself manifest early by rectal hemorrhage is cancer. It is in this

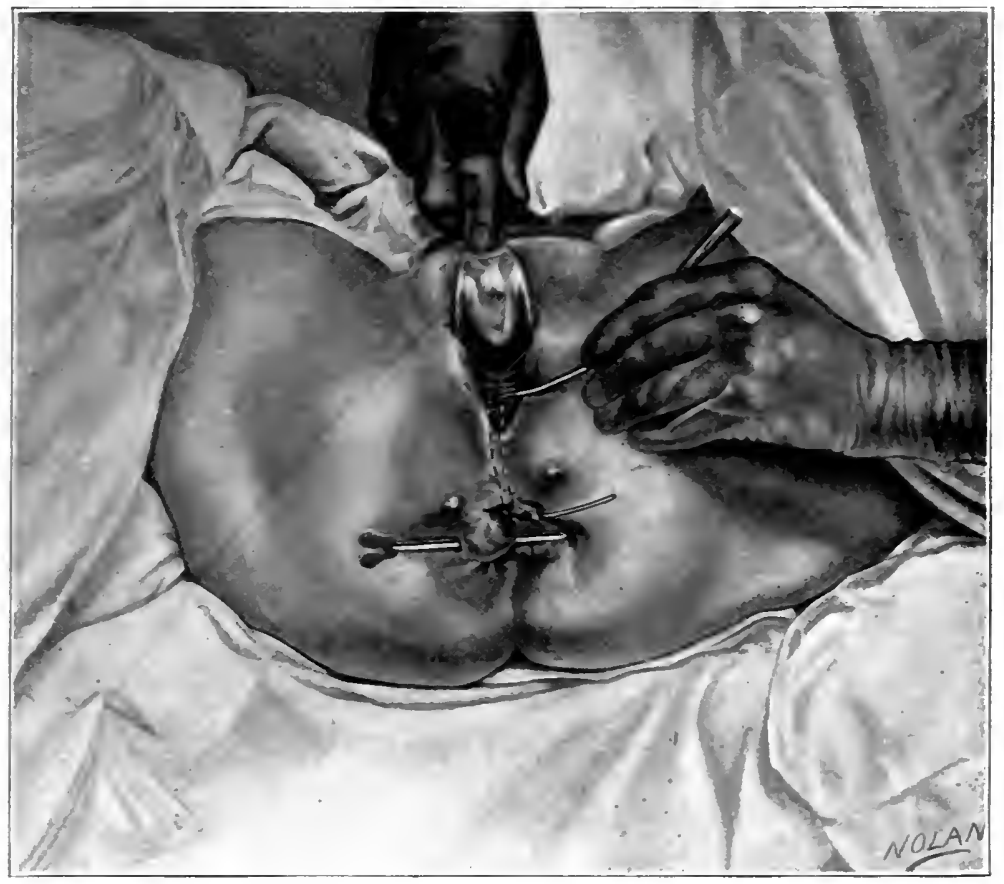

Fig. 120.

\section{Cancer of the Rectum, With Multiple Fistulae.}

This drawing. made from a photograph of a case referred to the author, tells a pathetic story. The patient, a woman aged 52, suffering from various digestive disturbances and the appearance of blood with the stool, made her own diagnosis of "bleeding piles;" after six months of self-treatment she consulted an irregular advertising quack, who confirmed her diagnosis of "hemorrhoids," and proceeded to "absorb the growth by electricity." When her money ran out she was sent home "cured." Her condition one month later, when seen by the author. is illustrated above. The area of infiltration involved the entire anus. posterior wall of the vagina, and all of the perineal body between. Her perineum was riddled with abscesses and fistulae. The rectum and ragina communicated through a large recto-vaginal fistula, and the posterior wall of the bladder was infiltrated. The case was hopeless and she died shortly afterwards. 
condition, above all other's, where an early complete proctologic and sigmoidoscopic examination will achieve brilliant results, if the findings therefrom, will bring about an early operation for the removal of the growth. It is the same with malignant diseases in this part of the body as in all others; if the surgeon can only get at them early enough to thoroughly eradicate, he can relieve them with a pretty definite hope of permanent cure.

Inasmuch as rectal cancer most frequently occurs in the lower part of the organ, the early operation and complete removal is productive of much good. Some of the early symptoms of commencing cancer of the rectum or sigmoid are flatulence with colicky pains; diarrhoea alternating with constipation; tenesmus; increased mucous discharge, which is usually offensive in odor, and hemor. rhage. This hemorrhage is very slight at first, often showing a few blood streaks with the mucous, or small passages of blood either with the stool or occasionally between bowel movements. The nearer to the anus the cancer is located, the earlier in the disease the hemorrhage, on account of the traumatism to the growth caused by the passage of the feces. Cachexia, loss of weight and impairment of general health is not an early sign of rectal cancer. The indican reaction is usually present in urine in cancer, while it is absent in ordinary diarrhoea.

Diarrhoea which persists for some time, which is ac. companied by the presence of blood, however slight: should be regarded as suspicious and the patient carefully watched. When one considers that 50 per cent of all cancer occurs in the gastro-intestinal tract, and that 
16 per cent of all cancers of the digestive tract occur primarily either in the rectum or sigmoid flexure; one commences to realize the importance of examining every

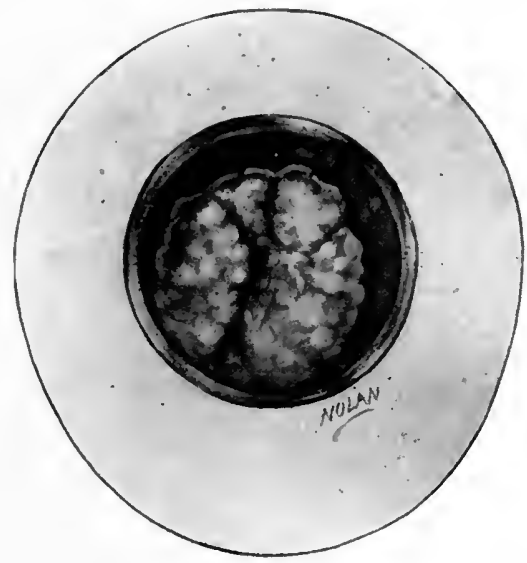

Fig. 121.

Proctoscopic View of Carcinoma Situated Just Below the Juncture of Rectum and Sigmoid.

(Drawn through the proctoscope. Author's case.)

case which presents a history of rectal hemorrhage, however slight, no matter the age or general appearance of the patient.

Well authenticated cases of cancer of the rectum have been found in cases as young as fifteen years of age. To show how much more frequently cancer is prone to locate in this part of the body than is generally supposed, it may be stated that Boas found in 500 cases of cancer of the di. gestive tract, 83 cases of cancer of the rectum. In the personal practice of the author, very frequently patients are brought in by practitioners, many of whom really try to do conscientious work, with unsuspected cancer of the 
rectum. Many of these patients are in the forties, present robust appearance and come with a history of some bleeding from the rectum from which they make their

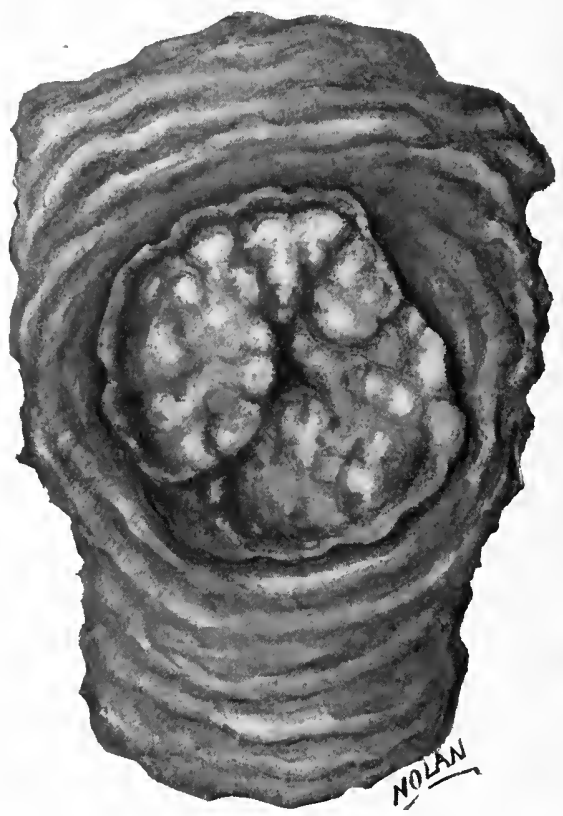

Fig. 122.

The Carcinoma Shown in the Preceding Illustration. Drawn from the specimen removed by operation.

own diagnosis of "bleeding piles." They also complain of some disturbance of bowel movements, either constipation or diarrhoea, and disturbed gastric and intestinal digestion and occasionally a not very well defined aching in the sacral region.

In many of these cases proctoscopic and sigmoidoscopic examination lias demonstrated the presence of cancer of the rectum, so far advanced, as to cause almost complete occlusion of the lumen of the bowel, and too 
far advanced to extirpate with any hope of cure. It is the unfortunate experience of many proctologists to be

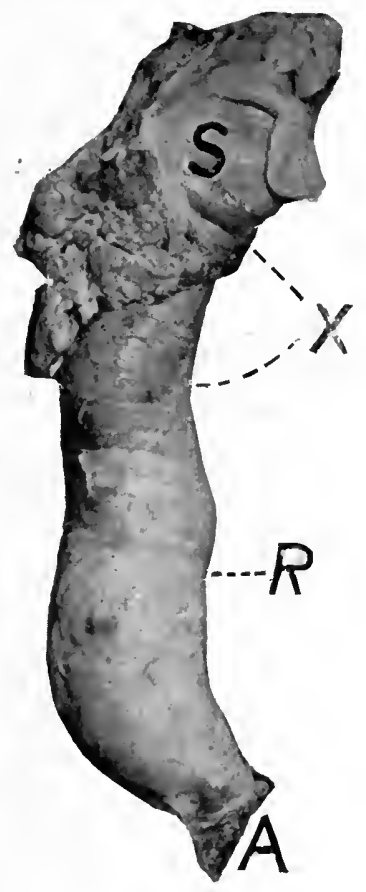

Fig. 123.

Cancer of the Rectum.

Photograph of specimen removed by the author.

This specimen, which includes the entire rectum and lower portion of the sigmoid, being eleven inches in length, was removed by the author by the perineal method, the sphincters being preserved. This case well illustrates the value of early diagnosis and prompt operative interference in cancer of the rectum. The patient, aged 50, suffered from gradually increasing disturbances of the digestive functions for about six months. The symptoms gradually grew worse and she noticed that her stools were becoming smaller in calibre and accompanied by a small quantity of blood. She consulted her physician, thinking she had hemorrhoids. He immediately made a proctoscopic examination and discovered just below and extending to the recto-sigmoidal juncture, a crater-like ulceration with raised edges, projecting into the lumen of the bowel. A diagnosis of rectal cancer was made and the case referred to the author for operation. There was no extra-rectal involvement and the complete extirpation of the diseased rectum and lower sigmoid was followed by a rapid recovery of the patient. 
called upon to inform many of these patients of their hopelessness, and it is with the hope of bringing the profession in general to realize the importance of examination of the rectal cavity in all cases presenting the symptoms just mentioned above; that so much stress is being laid on the importance of early examination of the rectum by the general practitioner.

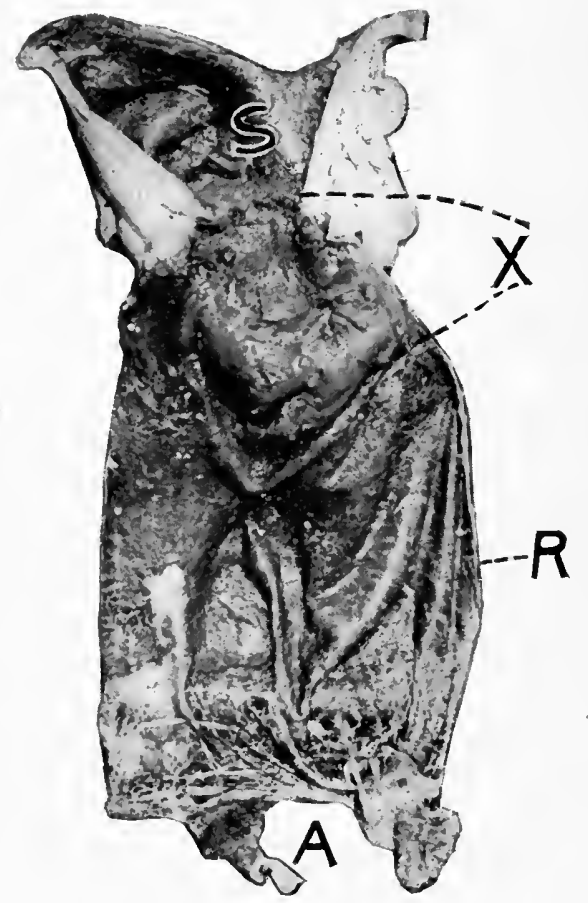

Fig. 124.

Cancer of the Rectum.

Same as the preceding. Interior view of the specimen.

A. Point of amputation from the anus.

R. Rectum.

$X$. Cancer.

S. Sigmoid flexure.

The lettering on the preceding specimen corresponds to the above. 
Cases of ulceration of the bowel involving more than one circumscribed area which have become chronic, as well as the very extensive ulcerations due to the specific infections like tuberculosis and syphilis, are not suitable cases for office or local treatment. It has been found in the experience of most proctologists that the only satisfactory way by which such cases may be cured, is by "sidetracking" the fecal current by means of a temporary colostomy. This removes the mechanical as well as the bacterial irritation from the ulcerated surfaces and puts the parts at rest; after which irrigations and other suitable therapeutic measures can be applied from above, as well as below. These cases, however, require more or less confinement to bed or to the house, and are best treated only in the surroundings which the modern hospital can best supply.

While it is true that colostomy can be performed under local anesthesia, as the author has demonstrated in several cases; it is hardly to be advised to be performed by the general practitioner or included in the same class as the operative measures or diseases mentioned in the foregoing chapters.

No case of stricture of the rectum should be treated whether by dilatation, incision, or electricity in office practice, unless it is situated within the first two inches of the ano-rectal canal, and is not smaller in calibre than the circumference of a No. 10 Wales bougie. Even then, its situation, consistency, structure and relation to the rectal walls and impinging organs, should be definitely ascertained by digital and instrumental, as well as ocular examination. Great caution should be observed in 
using forcible dilatation in any case of stricture of the rectum, no matter how elastic the stricture may seem. Accidents have been reported where the rectum has been torn through, and the peritoneal cavity entered with fa. tal result, from the simple dilatation of large calibred strictures by means of the Wales bougie. Cases of "stricture" due to musual infiltration of one of Houston's valves, or strictures of the umbrella type can be easily divided by means of the author's rubber ligature operation, as applied to hypertrophied rectal valves.

Where the administration of nitrous oxide is so easy, and attended with practically no danger, its use is to be advocated in those cases where operation of a few min. utes' (not exceeding twenty-five) duration is all that is required, for which general anesthesia is absolutely necessary.

While, as has been pointed out in a preceding chapter, some circum-anal and peri-rectal abscesses are amenable to treatment, within certain limitations, under local anes. thesia; abscess formation may go on to such a point, that it is absolutely necessary to do a more extensive operation than is possible under local anesthesia. Cerrainly no abscess which extends above the levator ani muscle should ever be opened under local anesthesia; nor any alsscess in the ischio-rectal region, in which there is any doubt as to the operator's ability to obtain a large and free drainage opening by means of incision without curetting. Owing to the ease with which an absress may extend, and the rapidity with which it enlarges in the ischio-rectal region, it is a safe plan not to attempt to open the abscess under local anesthesia, if it has become 
larger than a hen's egg in size, unless a definite point of fluctuation and softening can be detected at a point, well ontside of the sphincters.

No case of fistula-in-ano which has more than one channel or whose limits camnot be definitely made out by digital examination, should be opened under local anesthesia. Only the simple, direct, complete, or blind external, blind internal, or sub-mucons fistulae, are amenable to operation under local anesthesia and in cases of doubt, nitrous oxide or ether should be employed. One never can tell how high, or how extensive a dissection may be required for the complete removal of a fistulous tract, which is the ideal operation.

In operating for hemorrhoids under local anesthesia, one must be extremely careful in the selection of cases. Hemorrhoids complicated with fistula, extensive ulcera. tion, prolapse, or abscess, are best treated only under general anesthesia. External hemorrhoids and acnte thrombotic hemorrhoids can almost invariably be removed under local anesthesia, fully as satisfactorily as by the use of a general anesthetic. In the treatment of internal hemorrhoids and externo-internal hemorrhoids, however, there is a limit beyond which it is possible to go, but not wise.

The author in his practice has laid down the following rule: In all cases of internal hemorrhoids where not more than four separate hemorrhoidal tumors are present, whether prolapsing or not (see Fig. 85), operation under local anesthesia is the method of choice. Where more than four distinct hemorrhoidal tumors are present, or where there is much rectal prolapse complicating, their removal 
under nitrous oxide anesthesia is advised. Where, however, it is deemed unsafe or inexpedient, or where the patient absolutely refuses to take a general anesthetic; the more severe cases can be operated on under local anes. thesia by operating at several different sittings, remov. ing two or three hemorrhoids at a time, and then in a month or so removing more; eventually accomplishing the complete removal of all the hemorrhoids in three or four months and by as many operations. In some patients suffering from cardiac, pulmonary or renal disease, such a method may have to be followed where the admin. istration of a general anesthetic would be absolutely prohibited.

In cases suffering from interno-external hemorrhoids, where there are more than four separate tumors, their removal may be accomplished in two sittings by removing the external portions at one operation; when, with these out of the way, the internal ones can be removed with ease at the next sitting.

In prolapse of the rectum of the second degree, where the prolapsus only involves one-half of the circumference of the bowel, local anesthesia may be employed and the prolapsed portion ligated off in sections. As a general proposition, however, the author does not advise its use. Operations for prolapse have been done by some proctologists under local anestlesia, but the technique is rather crude, and the same satisfactory results cannot be obtained in this hurried method, as are possible under general anesthesia. In prolapse of the third degree (Fig. 111), local anesthesia is obviously contra-indicated, as the most successful operations for the reduction of complete 
prolapse is best accomplished by means of an abdominal operation.

'The removal of concretions from the rectum or sigmoid which are larger than one inch and a half in circumference, should not be attempted under local anesthesia, but can be done very nicely under the anesthesia produced by the administration of nitrous oxide. While almost any case of fecal impaction can be relieved under local anesthesia, as has been pointed out in Chapter $V$, there are some cases in which the procedure fatigues the patient so much, that the administration of a general anesthetic may by necessary, in order to successfully complete the operation.

Operations for fistula communicating betueen the rectum and other adjacent organs should never be attempted under local anesthesia; neither should the extensive use of the thermo cautery be attempted unless the patient is under profound anesthesia, if used at all. Before attempting any operation for relief of any pathological condition discovered in the anus or rectum, the absence of any other diseased condition higher up in the rectum, should first be demonstrated by careful proctologic and sigmoidoscopic examination. 


\section{CHAPTER XVII.}

\section{THE FECES AND THEIR CLINICAL EXAMINA- TION.}

By George Tr. Wagerer, M. D., Detroit, Mich.

It is smrprising that in the study of intestinal diseases so little attention has been given to the careful study of the stool. The study of the feces bears the same relation to the study of intestinal derangements, as the examination of the mine to the diagnosis of renal diseases.

I have, as far as possible, inclnded only the practical part of cropology, omitting those procedures that are of no particular benefit to clinical medicine and those requiring special laboratory training.

Under the term feces are comprised all those substances which, being formed from the food in the process of digestion, and mixed with the residues of the secretions of the alimentary canal, are finally expelled by the rectum.

Number of Stools.-The number of stools in 24 hours varies greatly in different persons, who are apparently in good health. One may have two to three bowel movements in 24 lours, while another may have but one in 48 hours, so it is important to ascertain the habitual num- 
ber of stools in every individual. There are rare instances in which one stool occurs only in 2 to 6 reeks. It is better, however, to take the general condition of the patient as a guide to the sufficiency of defecation. Some individuals will tolerate infrequent defecations white others would suffer from copremia under the same conditions.

Duration of Passage.--The question of the length of time required for the passage of food through the gastro-intestinal canal is a matter of much clinical inportance, ret little attention has been paid to the subject. It is quite as important to know the period of passage as to know how often the patient has a stool. A patient may have one stool a day, and yet have latent constipation, which gives rise to toxic symptoms. Whether latent constipation is present can only be determined by estimating the period of passage. In diarrhoea, by estimating the period of passage, it is possible to come upon an approximate idea of the seat of the disturbance producing the diarrhoea. If the period of passage is nearly normal, the trouble lies in the lower or middle portion of the large intestine, and peristalsis is probably not increased in the small intestine. Chronic colitis, with several watery movements a day, may be accompanied by a normal passage. The period is decidedly slortened if the inflammation is in the ascending colon or small bowel. Strauss used a test diet of 100 g'ms. of lean meat and found the normal period to be 10 to 20 hours. This was increased in cases of constipation as high as 60 hours. Maurel using a pure milk diet, gives the normal period 36 to 48 hours. In disease the shortest period was 4 homrs, 
and in such cases the bilirubin is found unaltered. The period of passage is very easily marked by giving a capsule of carmine with the meal and watching for the first red stool.

Amount.- The amount varies in different individuals, depending upon the character of the diet and the condition of the digestive organs. The quantity is increased by a diet rich in vegetables and starchy foods, and diminished by one rich in animal food.

The stool consists of the indigestible portion of the diet, the part of the diet undigested, bacteria and the secretion of the intestines and their associate glands. Cetti, who fasted 10 days, passed about 22 gms. of stool on the average per day. The normal amount varies between 100 and 200 gms. in 24 hours.

Consistency and Form.- The consistency of the stool depends chiefly upon the amount of water it contains, though there may be soft, thin stools due to abnormal amounts of fat or mucus. Increase of the fluid in the stools may be due to deficient absorption or to exudate or transudate from the mucous membrane. Increased peristalsis may cause watery stools through failure of absorption, while prolonged retention in the colon or rectum may result in hard, scybalous masses due to excessive absorption of water.

Odor.-The odor of the feces is, to a large extent, due to the presence of indol, skatol, sulphuretted hydrogen and methane.

Color of Stools.-The color of the feces varies according to the nature of the food ingested. The normal color is a dark brown. A diet consisting largely of meat 
gives an intensely brown stool, while a vegetable diet gives a more yellowish shade to the feces. A stool that has been exposed to the air is darker on the outside than

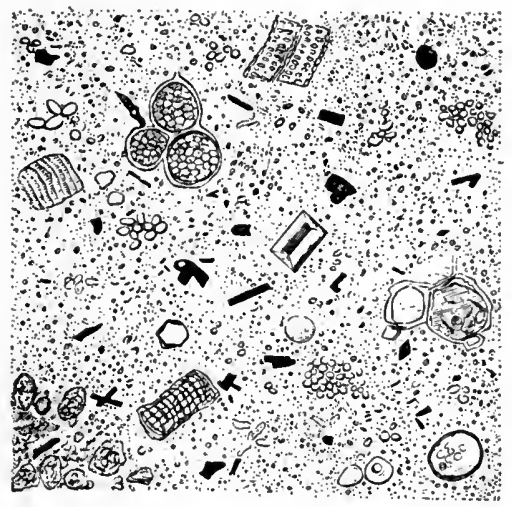

Fig. 125 .

Sulphide of Bismuth Crystals From the Stools.

(Eye piece III, objective $8 \mathrm{~A}$, Reichert.)

-Clinical Diagnosis: von Jaksch \& Cagney.

on the interior, owing to the process of oxidation. The presence of undigested fats gives a yellowish shade to the stool. If much blood is present the stool may be black or have a tarry appearance. Huckleberries and red wine produce a dark stool; chocolate and cocoa, gray; iron manganese and bismuth preparations a dark or black stool owing to the formation of the oxides of these metals. (Fig. 125.) Calomel causes a greenish stool (biliverdin), santonine, rhubarb and senna produce a yellow color.

Macroscopic Elements.-These are derived either from the food or from the intestinal apparatus itself. It is possible to find stones, cherry pits, 
grape seeds, skins of various berries or apples, pears ete., pieces of connective tissue, grains of cornin fact almost any part of the food if insufficiently masticated. The presence of casein in the stools of infants appears as small whitish lumps and can, as a rule, be easily recognized. Foreign bodies of almost every description that are not too large to swallow, may be found in the stools, especially in the stools of children and of the hysterical or of the insane; one may find buttons, coins, pins, false teeth, hair balls, etc.

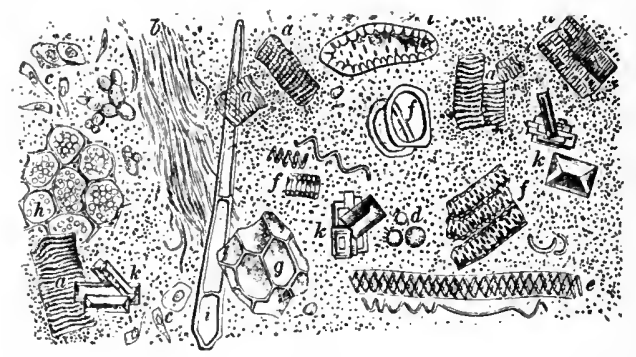

Fig. 126.

\section{Collective View of the Feces.}

(Eye piece III, objective $8 \mathrm{~A}$, Reichert).

$a$, Nuscle fibres; $b$, connective tissue; $c$, Epithelium; $d$, White blood corpuscles; $c$, Spiral cells; $f-i$, Various vegetable cells; $k$, Triple phosphate crystals in a mass of various micro-organisms; $l$, Diatoms.

-Clinical Diagnosis: von Jaksch \& Cagney.

Microscopically, may be seen indigestible and undigested portions of the food as well as substances thrown off by the mucous membrane of the intestines. Thus, stareh granules and remnants of chlorophyll, muscle fibre, elastic tissue fibres, connective tissne fibres, flakes of casein, white blood corpuscles, triple phosphate crystals, micro-organisms, ete., may be seen. (Fig. 126.) 
The Clinical Examination of the Stools. In order to make the clinical examination of the stools of benefit and satisfactory, we must have a standard for comparison. Schmidt, of Dresden, has formulated a diet to meet this requirement and it, or some modification, is now in general use by those following this line of work. There are two conditions for the satisfactory clinical examination of the feces.

1. A knowledge of what a normal stool sliould be under a certain diet.

2. The methods of examination must be as simple as possible.

1. The test diet--The requirements are:

(a) That it must be nutritious enough to furnish calories sufficient for the body's need.

(b) It must consist of such articles of food as can be obtained in any household.

(c) It must contain a constant amount of certain articles, so that variation in digestion and absorption can be detected in the stool.

Schmidt's diet is as follows: 1.5 litres of milk, 100 gms. Zweiback, 2 eggs, $50 \mathrm{gms}$. butter, $125 \mathrm{gms}$. very rare or raw beef, 190 gms. potato, and gruel from $60 \mathrm{gms}$. oatmeal and 20 gms. sugar.

This may be divided as follows:

Breakfast-Two eggs, half liter or two glasses of milk, one-third the amount of Zweiback and butter, or two slices of well toasted bread, with butter, and the oat meal and sugar.

Dinner-The steak and potatoes one-third Zweiback and butter and two glasses of milk. 
Supper-Two glasses of milk and the remainder of toast or ' Kweiback and butter.

The amounts of each article should be measured or weighed accurately and the beginning of the test diet marked by giving a capsule containing carmine or charcoal, preferably the latter because carmine would interfere with the color reaction in case an examination is made for blood in the stool. 'This diet should be given for several days. The first blask stool will denote the length of time required for the passage of food through the gastro-intestinal tract. The examination of the stool consists of the following steps: The consistency, color, and smell must be olsserved. Then a piece of formed stool the size of a walnut or an equivalent amount of liquid feces is rubbed up in a mortar with distilled water until it is quite smooth and liquid. Part of this is poured upon a glass plate or a Petri dish, put over a dark background and examined in a good light.

In normal digestion, very little should be seen by the raked eye except small brown points (oatmeal), and occasionally sago-like grains that look like mucus, but which the microscope shows to be grains of potato.

Pathologically, there may be:

1. Mucus in large or small flakes which is not affected by rubbing up in the mortar. The smaller the flakes the harder they are to recognize. It appears as glassy translucent flakes, often stained yellow by bile pigment. If at all doubtful, the microscopic examination will clear it up.

2. Pus, blood if considerable, can be easily detected, as can also parasites, stones and foreign bodies. 
3. Remnants of muscle fibre appear as small, reddish brown threads or small irregular hmps. When they can be easily seen by the naked eye and are quite numerous, it shows impairment of intestinal digestion.

4. Remnants of connective tissue and sinew from the beefsteak can be detected from the mucus by their toughness and whitish-yellow color. If in doubt, a piece may be put on a slide with a drop of acetic acid and examined with the microscope. The connective tissue loses its fibrous structure while the mucus becomes more threadlike. Small pieces of connective tissue can be found in normal stools, but when they are numerous and lalge their presence indicates the impairment of gastric dig'estion.

5. Remnants of potato look like grains of boiled tapioca and may be confused with mucus. Any doubt of the nature of the particles can be cleared up by the microscope.

6. Large crystals of acid phosphate of ammonium and magnesium occur in foul stools, and can be easily recognized by their shape and chemical reaction. (Solubility in all acids.)

For microscopic examination, prepare three slides from the liquid feces.

The first-a drop of the material to be examined under high and low power.

The second slide-mix a drop of the material with a drop of acetic acid (U. S. P), heating it to the boiling point, then put on the cover glass.

The third slide-a drop of the material with a drop of weak Lugol solution (Iodine 1, K. I. 2, Water 50). 


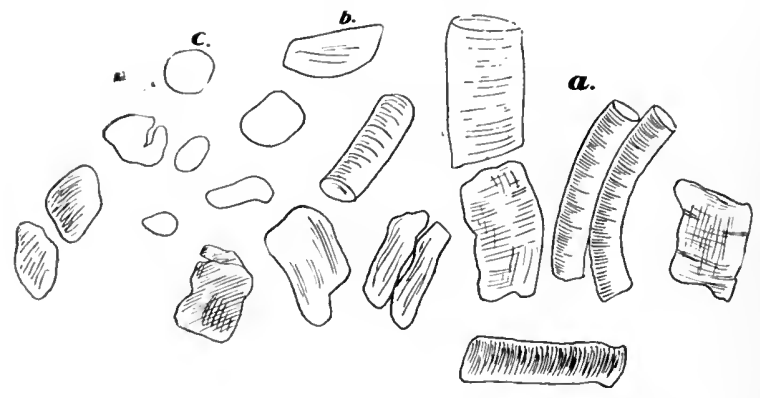

Fig. 127.

Muscle Remnants in Feces.

(Leitz objective VII.)

$a$, large; $b$, medium; and $c$, small fragments.

-From Schmidt \& Strasburger.

Normal Stool.-Slide one:

(a) Single small muscle fibres, colored yellow, usually with a cross striation. (Fig. 127.)

(b) Small and large yellow crystals of salts of fatty acids.

(c) Colorless particles of soap (gray).

(d) Single potato cells.

(e) Particles of oatmeal.

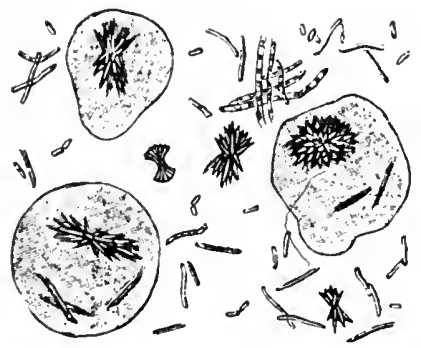

Fig. 128.

Haematoidin Crystals from Acholic Stools.

(Eye piece 11I, objective $8 \mathrm{~A}$, Reichert.) 
In the second slide a general idea of the fat content of the stool can be obtained. Upon cooling, small flakes of fat acids can be seen. The large crystals of salts of fatty acids and the soap are broken up by the acetic acid, and fat acids are liberated. If the slide is heated again and examined while hot, the fat acids will be seen to run together in drops, which, as the slide cools, break suddenly apart.

In the third slide, there should be violet-blue grains in some of the potato cells, and small single blue points, probably fungi spores.

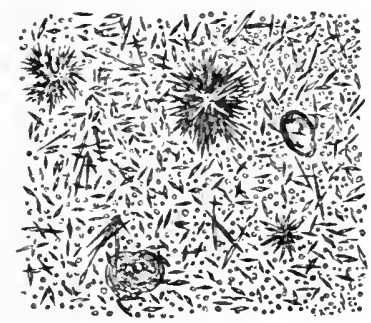

Fig. 129.

Acholic Stools.

(Eye piece III, objective 1-15, oil immersion, Reichert, Abbe's mirror, narrow diaphragm.) —von Jaksch \& Cagney.

Pathologically There May Be.-Slide 1:

(a) Muscle fibre in excess, perhaps with yellow nuclei.

(b) Neutral fat drops or fatty acids in crystals.

(c) An excess of potato cells with more or less well preserved contents.

(d) Parasite eggs, mucus, connective tissue, pus, etc. Slide 2.-Fat acid flakes in excess.

Slide 3.-Blue starch grains in potato cells or free oatmeal cells, fungus spores or mycelia. 


\section{Chemical Examination.}

The Reaction.-The reaction of the stool is hard to get with litmus paper, but can be easily obtained by dropping a little softened fecal matter into five or ten c.c. of a weak, watery solution of litmus, shaking it and noticing the change. It is well to use another test tube with the litmus solution only, as a control. The test should always be made with freshly passed feces, inasmuch as the reaction of the feces may change upon standing.

The Sublimate Test.-Consists of taking of a few c.c of the liquid feces and mixing it with an equal amount of 25 per cent watery solution of mercuric chloride. A normal stool will turn a pinkish-red, indicating the presence of hydro-bilirubin, which will be more intense the fresher the material. A green color, even if it is detected microscopically, is pathologic and indicates unchanged bile pigment.

Fermentation Test.-About $5 \mathrm{gms}$. of fresh formed feces is taken, or an equivalent amount of thinner material. Steele's Fermentation Apparatus, modification of Strasburger's is used. It is constructed of perforated rubber corks, bent glass tubing, and two test tubes of 30 c.c. capacity. (Fig. 130.) A small glass tube beam runs up to the top of the test tube $\mathrm{C}$ to allow for the escape of air.

The stool is rubbed up with sterile water and poured into the main bottle $A$. This is filled with sterile water; tube $\mathrm{B}$ is filled with water and fitted in place, and tube $\mathrm{C}$ is then fitted on empty. The reaction is carefully noted before the test is started. The apparatus is then stood 
in a warm place for 24 hours, best in an incubator at 37 degrees, (centigrade). If gas forms by fermentation in $A$, it will rise into $B$ and the amount will be indicated by the water displaced into $\mathrm{C}$. Normally, the fermentation test should show practically no gas, and the original reac-

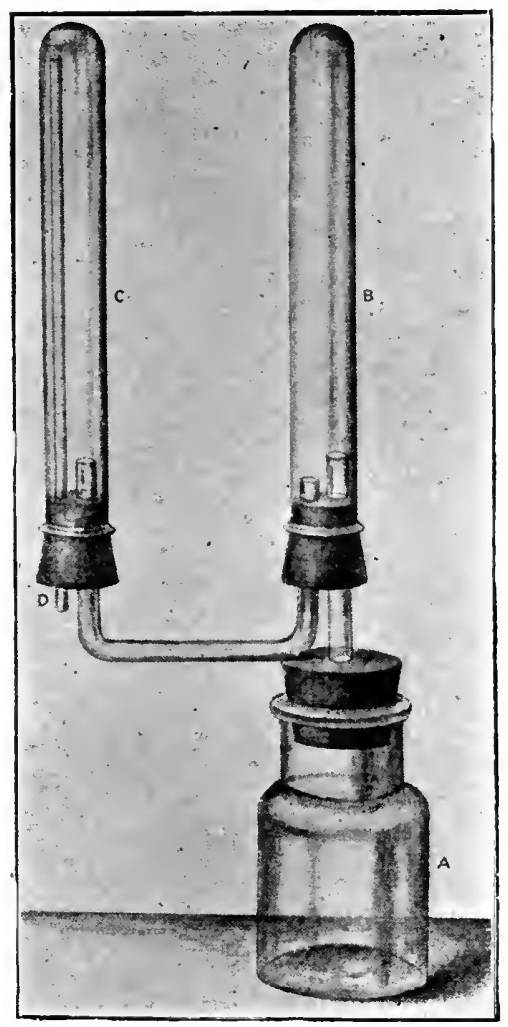

Fing. 130.

Steele's Modification of Strasburger's Fermentation Apparatus.

It is constructed of perforated rubber corks, bent glass tubing, and two test tubes, each of $30 \mathrm{cc}$. capacity. The small glass tube $D$ ruis up to the top of the test tube $C$ to allow for the escape of air, instead of the test tube being perforated, as in Strasburger's apparatus.

-Progressit'e Medicine, December, 1905. 
tion should be unchanged for 24 hours. If more than one-third of the tube $\mathrm{C}$ is filled, it is pathologic. If, then, the reaction is decidedly more acid, it is a carbohydrate fermentation; if alkaline and with a foul smell, it is a fermentation of albumins.

Estimation of Lost Albumin or Albumin Residue.

A qualitative test may be made as follows:

A softened portion of the stool is filtered, the filtrate shaken with silicon and re-filtered, then it is saturated with acetic acjd to bring down the nucleo-proteids, after filtration a drop of ferrocyanide solution is added. A decided precipitate indicates alloumin.

It was formerly thought that a positive test showed a diminntion of albumin digestion, but the work of recent investigators would indicate that this is not the case. Lnder pathologic conditions, the nucleo-proteids may be decidedly increased, although their presence is not characteristic of any particular disease. Other forms of albumin are rarely found in the feces, even after the ingestion of excessive amounts. The occurrence of albumin in the feces of adnlts is almost always associated with diarrhoea and usually with an excessive formation of mucus. It usually is serum albumin much less frequently albumoses. Such "lost albumin", in the stools indicate severe anatomical changes in the bowel but usually not disturbance of absorption. The albumin under these circumstances cones from the intestinal wall, and sometimes a part of them may be digested by the intestinal ferments into albumoses. 


\section{Clinical Significance of Test.}

Mucus.-There are two conditions in which the presence of mucus in the stools has no significance: When hard, dry masses of feces are covered with thin mucus, without evidence of rectal inflammation, and when it is discharged in casts, the so-called mucus colic. Otherwise it indicates inflammation of the intestinal mucous mem-

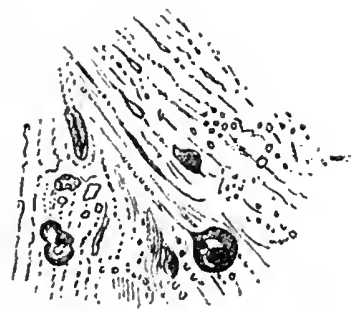

Fig. 131.

Mucus Shreds.

- From Schmidt \& Strasburger.

brane. If it is densely impregnated with bacteria, food remmants, and detritus the origin of the inflammation is probably high up in the intestine. (Figs. 131-132.)

Bilirubin discoloration affords no certain evidence of inflammation of the small intestine, but the presence of bilirubin granules and crystals in a cellular arrangement is suggestive.

The presence of semi-digested cells or of their nuclei indicates an origin high up in the bowel.

The presence of hyaline cells favors the assumption that an inflammation of the colon exists.

Bile Pigment.-A green color of part or all of a stool, by the sublimate test, is pathologic, except in chil- 
dren. It means a too short period of passage throngh the intestine, and that time for a normal reduction process of the bilirubin into hydro-bilirubin was lacking. A nor-

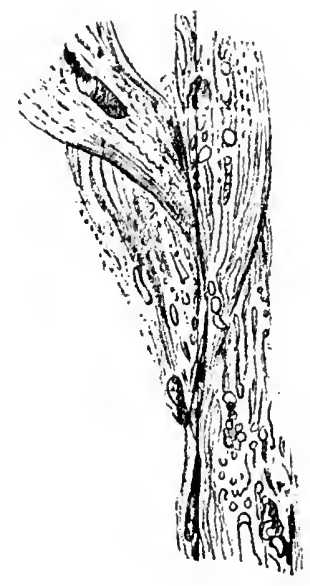

Fig. 132.

Mucus Shreds After the Addition of Acetic Acid.

-Urine and Feces in Diagnosis: Hensel, Weil, and Jelliffe.

mal fresh stool will give a pink color with mercuric chloride. If a color reaction of any kind is absent, it indicates a very fat stool, or an absence of bile in the intestime. (Fig. 1:9.)

The assumption of the temporary stoppage of the bile does not account for all of the cases of colorless feces which do not darken on exposure. The pathologic conditions in which colorless feces without jaundice may occur comprise defective supply of bile to the duodenum, intestinal catarrh, tubereulons abdominal disease, malignant disease of the intestine, septic diseases (especially those which affect the abdomen), chlorosis and leukemia. 
Fat.-It will need a little practice to tell, by the use of the diet, whether there is an increase of fat in the stool. As the normal amount of fat in the feces varies between wide limits only a considerable excess of fat can be detected.

Remnants of Meat.-Normally there should be only microscopic particles of connective tissue and muscle fibre. An excess of either is often visible to the naked eye, but need not be macroscopic to be pathologic.

Excess of Connective Tissue indicates insufficient gastric digestion, because such fibrous tissue is only digested by the gastric juice. The meat should be rare, to give this test its full value. If motility is increased, there nay be an excess of this in hyper-acidity.

Excess of Undigested Muscle Fibre, indicates intestinal indigestion and probably means trouble in the upper part of the small intestine; but whether the trouble is in the trypsin of the pancreatic secretion, or the activating principle (entero-kinase) of the intestinal juice, or in increased peristalsis, we can only judge from other symptoms. When the gastric juice fails to digest away the frame work of the muscle fibre, giving the intestinal juices no chance to do its work, eonnective tissue and muscle fibre are often found. This occurs often in acute gastrie catarrh.

Pathologic Carbohydrate Fermentation means poor starch digestion and indicates, as a rule, disturbance in the small intestine and usually is due to insufficiency of the succus entericus.

Pathologic Albumin Fermentation means a large residue of albumin in the feces and indicates usually some 
anatomical change in the mucous membrane of the small intestine.

Pus can be rarely recognized in the stool unless it comes from the lower part of the large bowel; if it comes from high up in the intestine it is rapidly changed.

Blood in the Stools.-The presence of blood in such quantities to be visible is considered in Chapter II, so I will only consider the so-called occult blood in the stools. The presence of occult bleeding from the gastrointestinal tract is a symptom of much importance, providing various sources of error can be eliminated. It has the same clinical significance as visible hemorrhage and its presence is of decided diagnostic value, chiefly in the detection of gastric or duodenal ulcer, or gastrointestinal cancer, because it oceurs with considerably more regularity and frequency in these affections. than in any other condition of the gastro-intestinal tract.

The value of this sign depends entirely upon the care with which the varions sources of error are eliminated, and if the reaction is positive will be of value in the diagnosis of cancer or ulcer of the gastro-intestinal tract only when sources of bleeding that have no significance are excluded. On the other hand, after repeated examinations, occult blood is not found, then cancer or ulcer can be excluded. Since the test is very sensitve (very small amounts can be detected), the chance for error in determining the origin of the hemorrhage is greater than in large and visible hemorrhages. Observations have shown a positive reaction in the feces on the ingestion of 0.5 gms. of blood. It is possible to exclude the source of the blood when in the lower bowel by the use of the procto- 
scope, etc. Tuberculous ulcer, typhoid fever, hemorrhoids, fissure and purpura can be easily excluded; other conditions, however, e. g. cirrhosis of the liver with slight symptoms may be the cause of error. Red beets, earmine, swallowed blood from any cause, hemoptysis, epistaxis, menstruation, cirrhosis of the liver, purpura, benign stenosis with stagnation, tuberculous enteritis, cancer of the gastro-intestinal tract, gastric or duodenal ulcer, typhoid ulcer, hemophilia. hemorrhoids, fissure, and fistula of the rectum is a partial list of conditions which may give a positive reaction with the various tests.

When testing for occult blood it is best to have the patient on a diet free from meats and meat juices and to give a good sized capsule of charcoal; the first black stool will mark the feces following the meat-free diet.

A number of different tests are used for the detection of occult blood; probably the Weber test, with its various modifications is the one most employed. It is well, however, to use a control test, preferably Klunge's aloin test. If both tests give a positive reaction, there is no doubt but that there is blood in the stools. The latter is not liable to be obseured by bile pigments or chlorophyll, in the ethereal extract, and is extremely delicate.

Webber's Test.-Take 2 or 3 gms. feces, mix thoroughly with 20 c.c of water; extract with 20 c.c ether to remove fats. Then use one-third the volume of acetic acid and shake well; add 10 c.c of ether and shake well. If ether doesn't come to the top soon, add a few drops of absolute alcohol. To 2 c.c of the ethereal extract, add 10 drops freshly prepared tr. guaiac and 10 to 20 drops of ozonized turpentine. Care must be taken that all uten- 
sils are absolutely clean and free from water. If blood is present, an intense blue color appears, gradually assuming a reddish violet tint.

Klunge's Aloin Test.-Take a small quantity of aloin, mix with 3 to 5 e.e of 70 per cent alcohol. Four to five c.c of acetic acid ethereal extract is tried with 20 to 30 drops of ozonized turpentine and 10 to 15 drops of the aloin solution. If blood is present a bright red color appears which turns to a cherry red on standing. If blood is not present, a yellow color remains for an hour or two, then Jecomes pink. It may take 15 or 20 minutes to get a positive reaction.

Holland's Modification of Webber's Test.-Instead of using ozonized turpentine, Holland used sodium perborate (Sherring) in tablet form; a few drops of the acetic acid ether mixture is placed upon a small piece of tablet of peiborate of sodium and a drop or two of the tincture of guaiac is cantiously brought into contact with it, preferably on a white plate. If blood is present, the perborate turns blue in a few minutes and remains blue until the drying of the tincture of guaiac leaves a yellow residne which changes the blue to green. If the proportion of blood is small the perborate turns a pale blue, which turns green as the guaiac dries.

Benzidin Test.-A little benzidin and abont 2 c.e glacial acetic acid are shaken up together and set aside for the benzidin to dissolve. A piece of feces the size of a bean is stirred into a test tube about one-fifth full of water ; the tube is plugged with cotton and the suspension of fecal matter is heated to a boiling point over a flame. About 10 or 12 drops of the concentrated benzi- 
din solution are poured into another test tube, from 2.5 to 2 c.c of a three per cent solution of peroxide of hydrogen added. One or two drops of the boiled suspension of feces is then added to this mixture. If blood is present in the feces, this brownish fluid turns green or blue; the more blood the more the test inclines to blue. The color reaction occurs within two minutes in the presence of blood and turns to a dirty purple in five to fifteen minutes. If there is no blood present the dirty brown color remains unaltered.

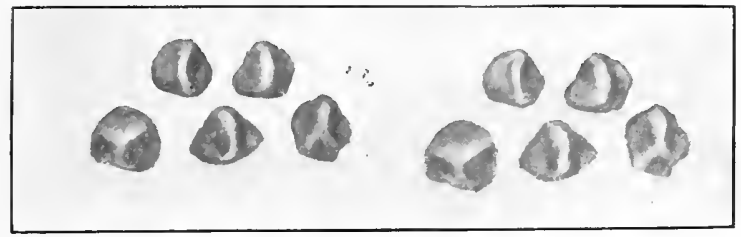

Fig. 133.

Gallstones.

Gall Stones.-In cases of colicky abdominal pain, the feces should always be examined for biliary concretions. 'The best way to search for gall stones is to put the feces in a fine sieve and wash the stool with running water from a fancet, if possible. The concretions vary in size from as small as the liead of a pin to the size of a pigeon's egg. They may be seen as small crumbling masses or as hard stones presenting an irregular contour or as smooth facets. (Fig. 133.) 'The larger stones are not passed by the bowel unless perforation has occurred into the intestine. The composition of the calculi varies. Some are composed of cholesterin; some of inspissated bile, and other's of calcareous salts. Those composed of cholesterin are the 
most common and are somewhat soft and white, greyish, bluish or greenish in color. I think that the consensus of opinion inclines to the belief that the nucleus of the majority of gall stones is clumps of bacteria, either bacilli coli communis or typhoid bacilli, although it may be composed of earthy sulphates or phosphates. Calculi which consist largely of biliary pigments are brown in color, hard and heavier than water; those composed of calcarious salts are generally irregular and rough.

Intestinal Concretions, or Enteroliths, are rare. At times their nucleus consists of some foreign body like a fruit seed, upon which calcium and magnesium salts have become deposited.

Intestinal Sand is hard, gritty, pale brown to black in color, readily sinks in water, and is usually composed of the salts of calcium magnesium and ammonium. Sometimes silica is present.

\section{Animal Parasites.}

Pathologic Forms.-Of the protozoa, the amoebae (see ('hapter XIIJ), are the most important in the etiology of intestinal disease. It is possible to find amoebae in the stools of perfectly normal individuals, and they increase in number as the stools become more alkaline in reaction.

In certain forms of dysentery the amoebae coli occur in the stools in enormous numbers, chiefly embedded in the mucus. They are also found on pathologic examination in the ulcers in the intestines. In examining the stool must he fresh, as the amoebae very rapidly die off in a stool that has been preserved but a few hours. A particle of mucus, preferably blood streaked, is taken 
from a fresh stool and placed on a chemically clean slide or better, a warm stage. In adjusting the cover glass, a horse hair or some similar object shonld be placed be-

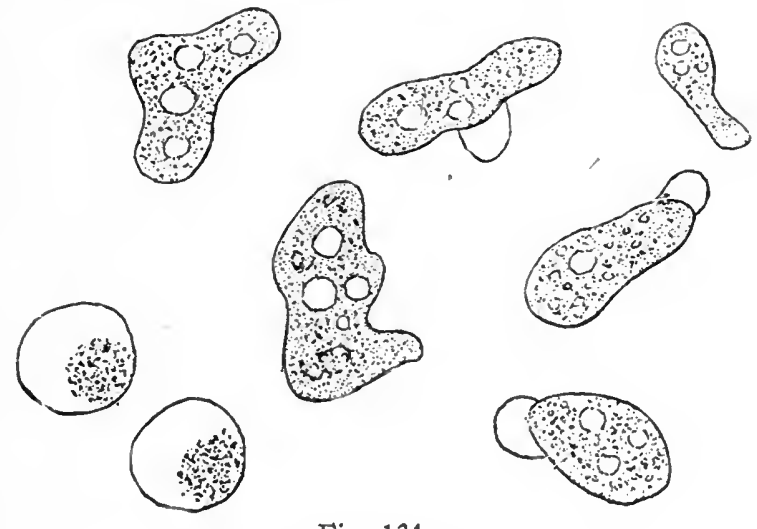

Fig. 134.

The Amoeba Coli.

-Clinical Diagnosis: Simon.

tween it and the slide, in order not to crush the organisms or interfere with their locomotion. Examine with a low power microscope. They are from 10 to 50 micro-millimetres in size. When at rest, their outline is, as a rule, circular or ovoid; but when in motion, they present one or more arm-like prolongations, "the pseudopods." The protoplasm ean be differentiated into a translucent, homogenous ectosare or mobile portion and a granular endosare containing the nucleus, vacuoles, and granules. (Fig. 134.) As a rule one or two large vacnoles are present, the edges of which are not infrequently surrounded by fine, dark granules.

Balantidium Coli.-Another form of protozoön, that is an etiologic factor in certain forms of dysentery is the balantidium coli. This organism is a harmless inhabitant 
of the colon of the pig, and it is supposed, is transferred to human boings through sausages. (Fig. 135.) The parasite is of oval shape, 60 to 100 microns long and 50 to 70

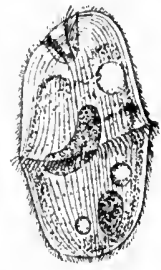

I

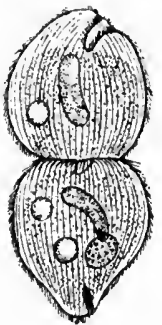

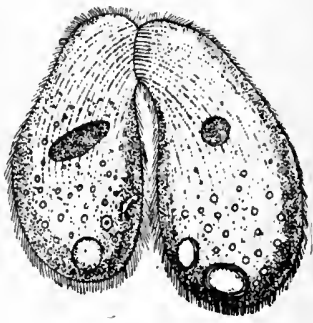

3

Fig. 135.

Balantidium Coli.

2. 1 and 2 stages of division. 3 Conjugation. (After Lenckart.) -Progressize Medicine, December, 1905.

broad, and is covered with cilia that are in rapid motion when the organism is alive. Ectosare and endosare are sharply differentiated. The endosare is granular and contains a kidney shaped nucleus, generally two contractile vacuoles and granular detritus. Motion is so rapid that it camnot be followed under the microscope. The protozoön dies very quickly and undergoes fragmentation.

There are other forms of protozoa, but their role in the etiology of intestinal diseases is not definitely settled.

\section{Worms.}

The diagnosis of helminthiasis from the stools may be very easy or it may require considerable painstaking research. If segments of the taenia pass in the stools, the diagnosis is quite evident. In other cases, a diagnosis 'an only be made by finding the ova in the feces. To examine for the ova, take a small amount of feces from 
different parts of the stool, dilute it very much with sterile water and centrifuge repeatedly. After each centrifugalization, the supernatent dirty water is thrown away and fresh water is added, the whole shaken up and again placed in the centrifuge, this to be repeated five $\mathrm{or}^{\circ}$ six times. In this way all bacteria, free coloring matter, light vegetable matter, etc., are gotten rid of and only heavier particles including any ova that may be present, will remain and can be easily and satisfactorily examined under a low power of the microscope. There is the left no obscuring clond of hacteria or fine g'ramlar debris, but insteal each ovmm, or muscle fibre, or crystal stands out sharp and clear.

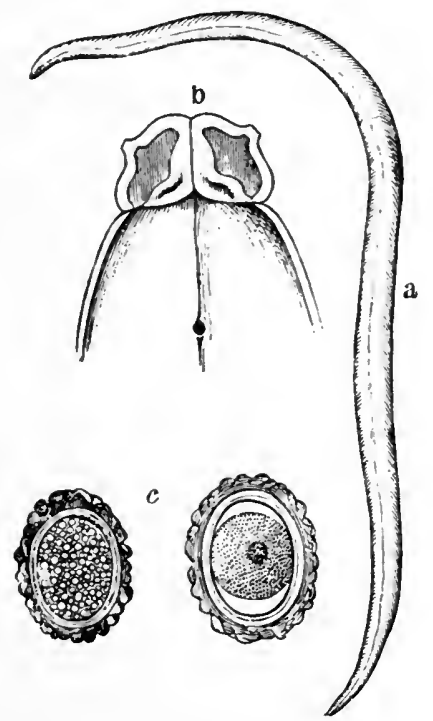

Fig. 136.

Ascaris Lumbricoides.

$a$, The Worm: $b$, Head: $c$, Eggr: $a$, half natural size: $b$, slightly magnified; $c$, eye piece I, objective 8a. Reichert.

-Clinical Diagnosis: von Jaksch \& Cagney. 
Nematodes. Nematodes are round worms. Those found in the human being are:

1. Ascaris Lumbricordes is the most common parasite of the human intestinal canal. 'They are found chiefly in the small intestines but may find their way into the stomach, the bile passages, or out at the anus. Clumps of them have been known to cause intestinal obstruction.

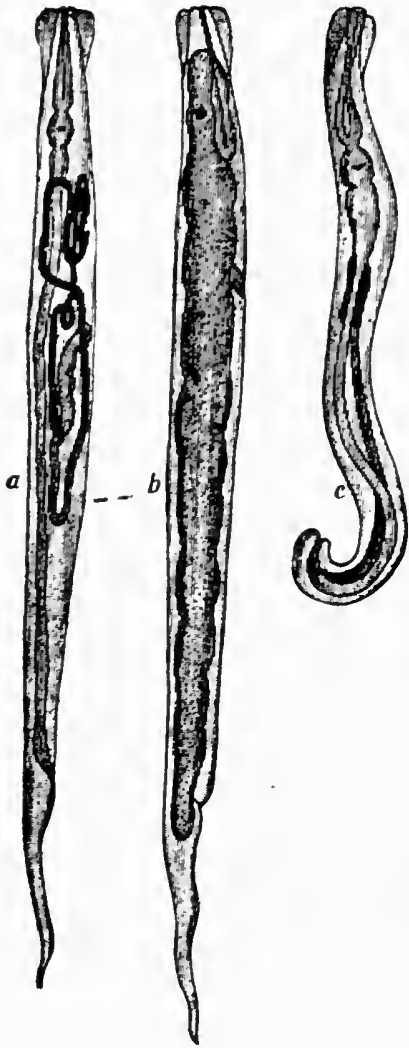

Fig. 137

Oxyuris Vermicularis.

$a$, sexually mature female; $b$, female filled with eggs; $c$, male. Magnification, 10. (After Heller, from Ziegler.) 
The worm is cylindrical, the male being from 10 to 25 c.m. in length, the female from 25 to $40 \mathrm{c.m}$. The head consists of three projections or lips, which are provided with suckers and fine teeth. The tail end of the male is rolled up on its ventral surface like a hook and is provided with papillae. The genital aperture of the female is situated directly behind the anterior third of the body. The egg's are yellowish brown in color, almost round, and measure $0.06 \mathrm{~mm}$. by $0.07 \mathrm{~mm}$. in size. They are surrounded by an irregular albuminous envelope which is covered by a tough shell; the contents are coarsely granular. (Fig. 136.)

2. Oxyuris Vermicularis.-(Common thread worm, teat worm, pin worm, etc.), is a very frequent parasite, especially in young children, often passing from the anus into the vulva in female children and setting up considerable irritation in the vagina. The male is $4 \mathrm{~mm}$; the female $10 \mathrm{~mm}$. long. At the head three lip-like pro-

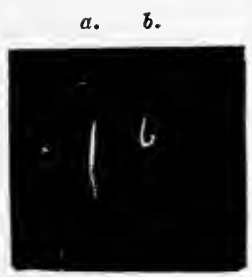

1

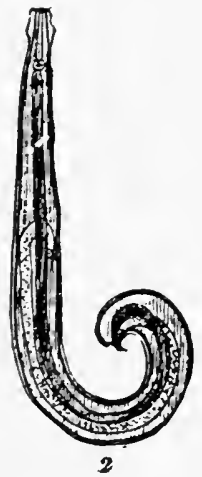

Fig. 138.

Oxyuris Vermicularis.

I. Oxyuris vermicularis; $a$, Mlale; b, Female; natural size. 2 , Magnified. -Urine and Feces in Diagnosis: Hensel, Weil \& Jelliffe. 
jections with lateral cuticular thickenings may be seen. The tail of the male is provided with six pairs of papillae and the female with two uteri. The eggs are 0.05 by 0.02 to $0.03 \mathrm{~mm}$. in size, and covered with a membrane showing a double or triple contour. In the interior, which is coarsely samular, the embryo are contained. The ova do not ocemr in the feces. (Figs. 137-138.)

Anchylostomum Duodeuale, or Dochimus Duodenalis, or Strongylus Duodrnalis is generally described in America as [ncinaria. It was formerly supposed that this parasite was found only in the Old World and only brought into this comntry, but it has been demonstrated that there are many endemic cases in our Sonthern Sitates.

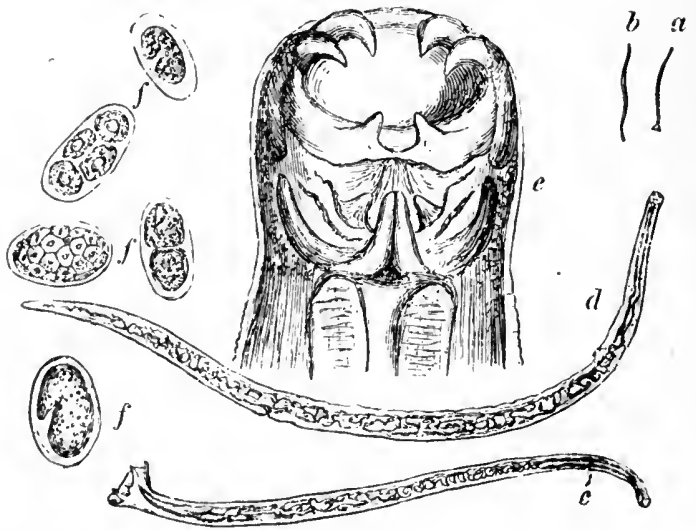

Fig. 139 .

Anchylostoma Duodenale.

a, Male (natural size).

$b$, Iiemale (natural size).

c, Male (magnified).

d, Female (magnificd).

c, Head (eyepiece II, objective C, Zeiss).

$f$, Liggs. 
There are certain differences between the American and Old World parasite.

Stiles, in Bulletin No. 10 Mygienic Laboratory, U.S. Public Health and Marine IIospital Service, gives the following description: Uncinaria duodenalis--"The OId World hook worn: Body eylindrical, somewhat attenuated anteriorly; bnecal cavity with two pairs of ventral teeth curved like hooks, and one pair of dorsal teeth directed backward; dorsal rib not projecting into cavity. Male 8 to $11 \mathrm{~mm}$. long, caudal bursae with dorso-median lobe and prominent lateral lobes united by a ventral and slender. Female, 10 to $11 \mathrm{~mm}$. long; vulva at or near posterior third of body. Eggs ellipsoid 52 to 60 micromillimetres by 32 micromillimetres laid in segmentation. Development direct without intervening host." (Fig. 139.)

Uncinaria Americana-"The New World hook worm of man, body cylindrical, somewhat attenuated anteriorly, buccal capsule with a dorsal pair of prominent semilunar plates or lips and a ventral pair of slightly developed lips of same nature; dorșal conical median tooth projects prominently into buccal cavity. Male $7 \mathrm{~mm}$. long, caudal bursae with short dorso-median lobe which often appears as if it were divided into two lobes, and with prominent lateral lobes united ventrally by an indistinct ventral lobe; common base of the dorsal and dorsolateral rays very short; dorsal ray divided to its base, its two branches being widely divergent, and their tips being bipartite; spicules long and slender. Female 9 to $11 \mathrm{~mm}$. long; vulva in anterior half of body but near equator. Egrgs ellipsoid, 64 to 76 micromillimetres long by 36 to 40 micromillimetres broad, in some cases parti- 
ally segmented in ntero; in others containing a fully developed embryo oviposited." 'The eggs of the American species are much larger than those of the Old World species. The eggs have a transparent shell with a linear contour and are often found in enormous quantities in the feces. A rather peculiar fact that the ova of uncinaria, although sticking closely to the glass slide, do not seem to adhere to any of the other constituents of the stool. When a drop of washed sediment feces is allowed to remain on the slide for a few minutes and then gently immersed in water and examined microscopically, the eggs are found adhering to the slide and all else has been washed away. In suspected cases where the diagnosis is difficult, a full dose of thymol may make it clear ; causing the appearance in the stool of the parasite which appears as a thread-like body, a half to three-quarters of an inch long, grayish red in color. Its habitat is the jejunum and duodenum. Infection takes place through contaminated drinking water.

For persons who are not in a position to make a microscopic examination, the blotting paper test will be found very useful. To make the test use only fresh feces. Place an ounce or more of the stool on a piece of white blotting paper, allowing it to remain for 20 to 60 minutes; remove the feces and examine the color of the stain. In about 75 per cent of the cases of medium or severe uncinariasis. the stain is a reddish brown resembling somewhat a blood stain. In making this test on anemic patients, hemorlhoids must be excluded.

Trichocephalus Dispar, or "Whip Worm," frequent in most parts of the world, gets its name from being 
formed like a whip, the lash end being the head end, while the tail end is very much thicker. The male measures $46 \mathrm{~mm}$. and the female $50 \mathrm{~mm}$. in length. The eggs

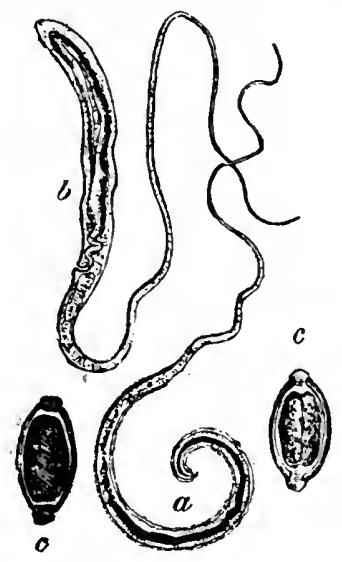

Fig. 140.

Trichocephalus Dispar.

$a$. Male; $b$. Female; $c$. Eggs; $a$. $b$., slightly magnified; $c$. (eye piece II, objective 8 a, Reichert.)

-ron Jaksch \& Cagney.

are brownish in color 0.05 by $0.06 \mathrm{~mm}$. in size, presenting a double contoured shell with a depression at each end, closed by a lid. The contents are coarsely granular. Its habitat is in the cectum; the living worm is rarely found in the feces. (Fig. 140.)

Trichina Spiralis.-The male is $1.5 \mathrm{~mm}$. in length, and the female $3 \mathrm{~mm}$. The male has four prominent papillae, situated between the conical protuberances at the extremity. The female's. sexual organs consist of a tubular ovary which is placed at the hinder part of the body and a tubular nterus with which the ovary communicates in front. Impregnation takes place in the intestine. The eggs develop into embryos while still in the 
uterus, and the newly born parasite almost immediately perforates the intestine and becomes embedded in the muscles of its host. The mode of infection is through

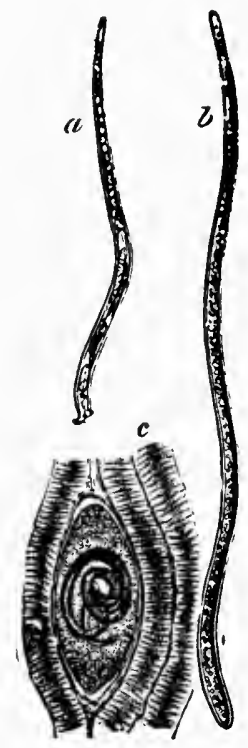

Fig. 141.

Trichinae.

$a$. Male, and $b$. Female Intestinal Trichinae, slightly magnified; $c$. Trichina of muscle (eye-piece III, objective IV, Reichert.)

-von Jaksch \& Cagney.

imperfectly cooked pork. Rarely is the parasite found in the stools. In suspected cases an anthelmintic may cause the expulsion of the mature worm in the stool. Eosinophilia is a constant accompaniment of the presence of trichina. (Fig. 141.)

Anguillula Intestinalis, is $2.25 \mathrm{~mm}$. in length and $0.04 \mathrm{~mm}$. in thickness at its middle. It has a triangular mouth closed by three lips. Its vulva lies at the junction 
of the middle with the posterior third. Its habitat is the small intestines. The eggs resemble those of anchylostoma duodenalis but are longer, more elliptical and

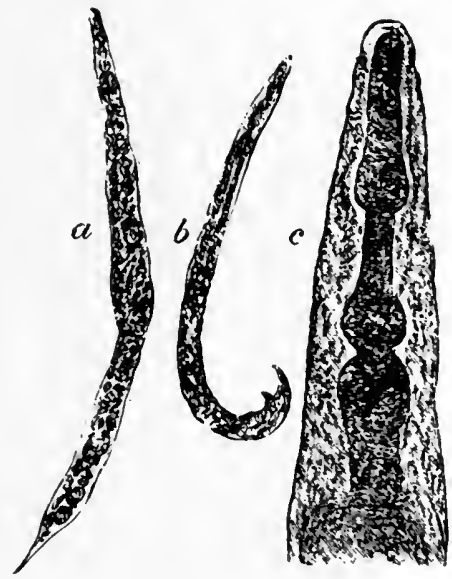

Fig. 142.

Anguillula Stercoralis.

$a$. Female; $b$. Male; $c$. Head (eye-pice II, objective 8 a, Reichert.) -von Jaksch \& Cagney.

pointed at the poles. In recent stools the larvae alone can be seen. When sexually mature, it is known as anguillula stercoralis; the body is round; it shows faint traces of transverse striation. The head is the form of a blunt cone and sessile on the body, and is furnished with two lateral jaws, each bearing a pair of teeth. The male is $0.88 \mathrm{~mm}$. and the female $1.2 \mathrm{~mm}$. long. Little is known concerning the manner of infection. Thayer reported the first case of infection by this worm in the U. S. (Fig. 142.)

Cestode Worms. Cestodes are popularly known as tape-worm. Externally they are long, flattened segmented worms. The head is derived from the embryo 
eontained in the flesh of the various domestic animals which are used as food. By budding it gives rise to all of the succeding segments which are morphologically the same, diminishing in size toward the head.

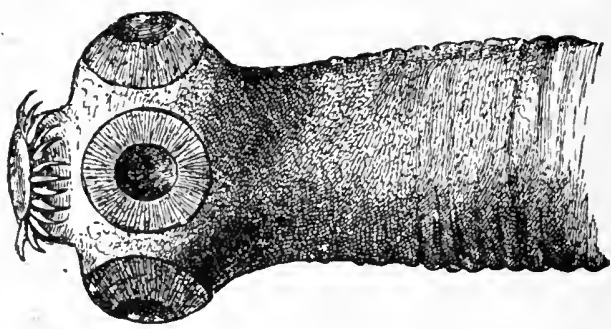

Fig. 143,

Head of Taenia Solium.

Head of Tacnia Solium. $\mathrm{x} 45$. (Letuckart.)

Taenia Solium.-The tape worm derived from pork may be two to three meters long. Head quadrilateral, about as large as a pin-head; it has four prominent suctorial dises, usually pigmented and between them a rounded elevation which is surrounded with about 26 looklets of different sizes, and is dark in color. This is succeded by a delicate thread-like neck about one inch in length and unjointed. The segments or proglottedes are short and relatively broad near the neck; the mature segments' average length is from 9 to $10 \mathrm{~mm}$. and breadth 6 to $7 \mathrm{~mm}$. and contains a uterus having five to seven branches. The ova are round and of a brownish color and surrounded with a thick radially striated membrane; in their interior the hooklets of the embryos can usually be made out. (Fig. 143.)

Taenia Saginata (Medio Cammulata).--The most frequent tape worm of Furope and America, infection taking place through measly beef. It is from 4 to 8 metres 
long. The head is surrounded with four large and usually black pigmented suckers, but is not provided with rostellum and is without a circle of hooklets. Segments

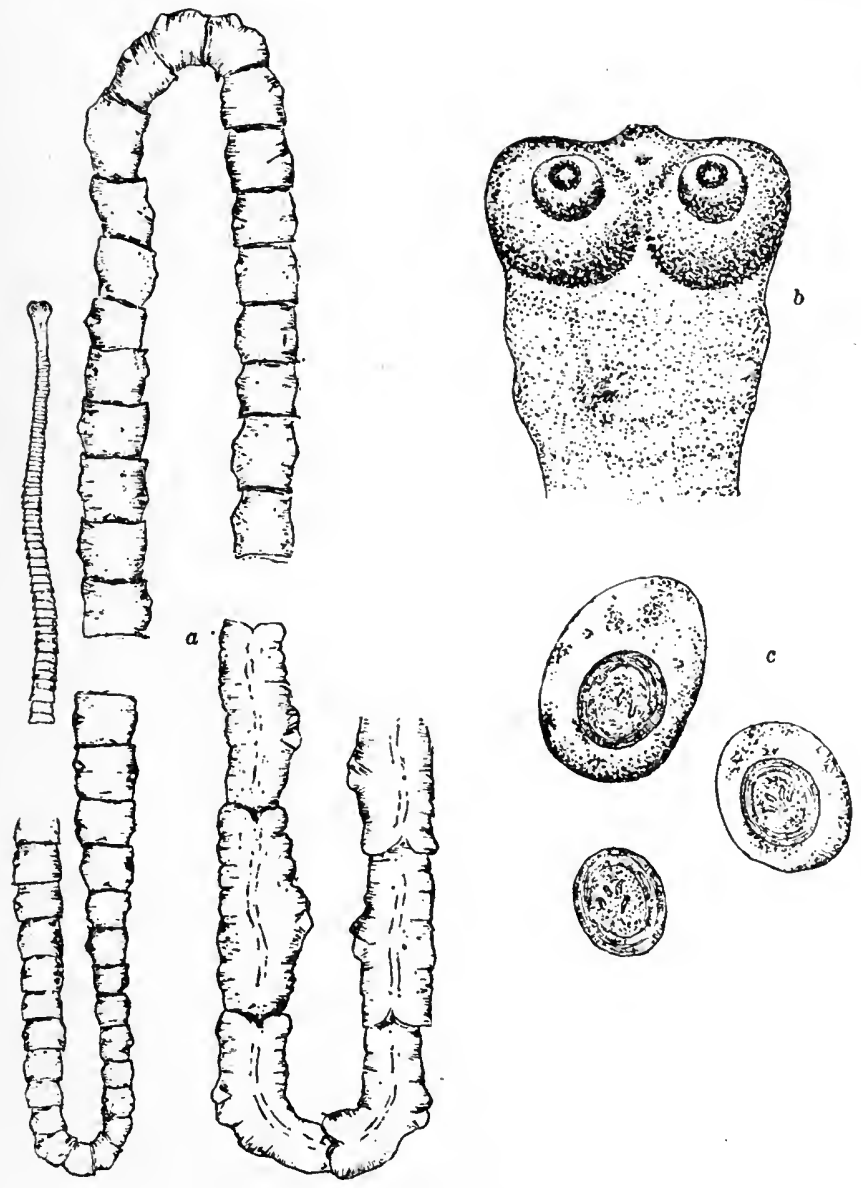

Fig. 144.

Taenia Saginata.

a. Natural size.

b. Head much enlarged.

c. Ova much enlarged. 
are quite thick and opaque, and each is provided with a very much branched uterus which opens laterally. The ova are elliptical in form, of a brown color, and usually enclosed in a distinct vitelline membrane. In the interior the embryos are seen embedded in a brown granular material. (Fig. 144.)

Taenia Nana.--Occurs rarely in America, mostly in Southern Italy. It is 7 to $15 \mathrm{~mm}$. long. It occurs in large numbers, and is usually located in the lower part of the lleum. It has four suckers and a crown of hooklets. Tht segments are of a yellowish color and about four times as broad as long. The uterus is oblong and contains numerous ova, having two distinct membranes. In the interior of the egg, may be seen the embryo already provided with five or six hooklets. Infection probably occurs from man to man. The parasites may be present in great numbers in the intestines, producing severe nervous symptoms such as epileptic seizures, insensibility, mental derangements, etc.
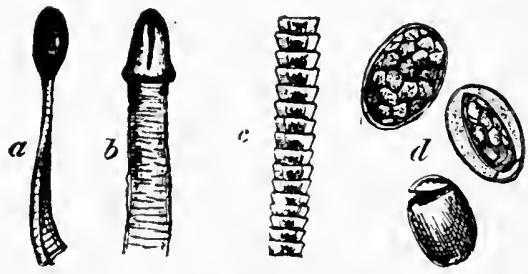

Fig. 145.

Head of Bothriocephalus Latus.

(Eye-piece III, objective IV, Reichert). $a$. Seen on edge; $b$. Seen on the flat; $c$. Proglottides; $d$. Eiggs.

-von Jaksch \& Cagney.

Bothriocephalus Latus.-The longest of the human tape worms has been found in the United States in only a few imported cases. The larvae have been found in 
various fishes. It is from five to eight metres long and tapers toward both extremities. The largest segments measure $3.5 \mathrm{~mm}$. in length, 10 to $12 \mathrm{~mm}$. in breadth. The head is ovoid, $25 \mathrm{~mm}$. long and $1.0 \mathrm{~mm}$. broad, somewhat flattened and provided in each lateral aspect with a groove-like sucking apparatus. The uterus is a slightly convoluted canal. The eggs are ovoid $0.07 \mathrm{~mm}$. by 0.045 $\mathrm{mm}$. and possess a thin brown capsule and open by a small lid at one end. This parasite may be the cause of severe anemia. (Fig. 145.)

\section{Character of Feces in Certain Intestinal Affections.}

Acute Intestinal Catarrh.-This follows the ingestion of excessive quantities of normal food or tainted food, beer and certain poisons, acids or alkalies, arsenic, corrosive sublimate, etc., when taken in proper quantities; also find it in cholera nostras, typhoid fever, severe malaria, also in diseases of heart, lungs and liver due to disturbance in circulation. The frequency of the stools depends largely upon the seat of the lesion; involvement of the large intestine, especially the transverse and descending colon causing the bowels to move more frequently than trouble higher up. There may be from 10 to 15 passages a day. On the other hand, isolated catarrh of the small intestine may exist without giving rise to diarrhoea. The stools at first are semi-solid but rapidly become liquid, often foul smelling and associated with gas. The higher in the bowel the lesion, the more odor and gas. The color varies from a light to a dark brown. If the trouble exists in the small bowel only, the stools are firm, formed, and contain particles of hyaline mucus 
visible only upon microscopic examination. It usually contains particles of undigested food. If the colon is affected, the stools are loose. Extensive involvement of the colon is usually accompanied by mucus in large quantities.

Chronic Inflammation of the Intestine.-May follow an acute attack or may follow some of the infectious diseases. Diarrhoea usually alternates with constipation. Rarer are continuous diarrhoea or constipation. The feces present the same characteristics as the acute inflammations.

Diphtheritic Enteritis.-Always diarrhoea, often with tenesmus. Stools fluid, with occasional passage of formed feces. They consist mostly of pus, blood and mucus, and some necrotic tissue may be found.

Mnco-Hembranous Colitis.-No frequency in number of stools; may have constipation. Stools are composed largely of tough leathery mucus which may present casts of the bowel. This may be transparent or gray and semi-opaque, or may be brown (from fecal matter), or red (blood).

Cholera Nostras.-An infectious disease affecting both the stomach and bowels. The stools are first feculant, but soon become colorless and more and more watery until they resemble the so-called "rice water" stools of Asiatic Cholera, and contain serum albumin and mucin.

Dysentery.-Stools are large and frequently composed of pus, mucus, and blood, fluid or semi-fluid, may find necrotic masses of mucous membrane.

Amoebic Dysentery.-Stools are frequent, fluid, and may contain large amounts of mucus, frequently stained 
CHARACTER OF FECES IN INTESTINAL AFFECTIONS. 363 with blood; reaction always alkaline. Microscopic examination of the fresh mucus shows epithelial and red blood cells and the amoeba.

Carcinoma of the Small Intestine.-The stools of which have no distinctive feature.

Carcinoma of the Rectum and Sigmoid is taken up elsewhere in this volmme. (Chapter XVI.) 


\section{INDEX}

A.

Abscess, ano-rectal, chapter on $\ldots \ldots \ldots \ldots 137,189,305,322$

-classification ........... 138

-general etio'ogy ......... 137

-ischio-rectal...146, 154, 165, 166

-diagnosis ........... 148

- - etiology ............ 147

-symptoms ........... 147

-treatment ............. 149

-incision ............... 149

-sub-mucous.....142, 154, 166, 617

一diagnosis ............. 143

-examination ........... 142

-symptoms ............ 142

-treatment .............. 143

-incision ............... 145

-sub-tegumentary.....140, 154, 165

- diagnosis .............. 142

-etiology ............... 141

-examination ........... 142

—symptoms ............. 141

-treatment ............. 14.

-tegumentary .........138, 165

- diagnosis .............. 140

- etiology .............. 13s

-treatment ............ 140

ADLER, L. H., Jr.......... 111

Albolene, liquid, 89, 100101 ,

$128,168,203,208,228,232,289$

ALLBUTT ...........249, 250

Alligator forceps.......... 67

Amoeba coli mitis (see parasites) $\ldots \ldots \ldots \ldots \ldots 251,346$

-dysenteriae (see parasites).. $.248,249,346$

Anal canal
Anal Fissure (see Fissure in Ano) ............... 122 Anal Fistula (see fistula in ano) ............... 152

Anal Papilla (see Papillae anal) .............209

Anatomy, chapter on........ 17

Anchylostoma duodenale..... 352

ANDREWS ............. 352

Anemia...........40, 181, 314

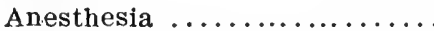

-local (see local anesthesia). 295

-general (see nitrous oxide).. 158

Anguillula intestinalis (see parasites) ............. 356

Animal parasites (see para-

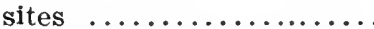

Ano-coccygeal ligament...... Anoscope (see HirschmanKelly)

Anoscope-Hirschman's . . . . 62, 156

Anoscopy ...........64, 186

Anus $\ldots \ldots \ldots \ldots \ldots \ldots \ldots \ldots \ldots$

-dilatation (see dilitationsphincter) $\ldots \ldots \ldots \ldots .18,20$

-eversion of ............. 54

-fissure of (see Fissure).... 12?

-ulcer of (see Ulcer) ......... 122

Appendicostomy ........... 282

Appendix vermiform....... 263

Appetite, loss of.......... 40

Artery (see Frontispiece)

-hemorrhoidal inferior....29, 30

-middle ............30, 31

-superior ............. 30

-iliac, internal.......... 31

-mesenteric, inferior....... 30 
Artery, pudic, internal....... —sacral, middle..........

-vesical .............. 30

Atresia ani vaginalis

- complete ..........74, 76

-incomplete .........75, 76

Ascaris Iumbricoides (see

worms ) ........... 349

Auto-intoxication ........40, 38

AYERS $\ldots \ldots \ldots \ldots \ldots \ldots \ldots .239$

B.

BALI, SIR CHARLES..26, 116, 119

BECK, EMIL G........... 169

Bismuth paste injections..... 169

Bleeding.......34, 98, 126, 180

$187,188,209,222,235,259,314$

Blood in the stool (see b!eed-

ing) .............. 342

-test for............332, 342

-Hollands ............... 344

-Klunges aloin............ 344

-Webber's .............. 343

Bothriocephalus latus (see parasites ) ...............360

Bougie, Wales......69, 311, 321

Bovinine $. \ldots \ldots \ldots \ldots \ldots .132,162$

c.

Canal, anal...........18, 126

Cancer, rectal....36, 181, 188,

$314,315,316,317,318,319,321$

Carcinoma, rectal (see cancer).

Cauterization of hemorrhoids.. 191

-electro of hemorrhoids....... 191

CETTI .............. 328

Chloretone.........112, 190, 297

Clamp and cautery.......... 204

Clover's crutch............ 56

Cocain ................296

Coccyx, examination of....58, 59

Colitis (see Proctitis and sig-

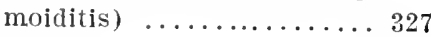

Colostomy ............. 321
Columns of Morgagni....... 23

Commissures, ana!....123, 124, 126

Concretions (see impaction).97, 221

- removal of (see Foreign Body) ...........325, 346

Condylomata (see Warts)....

...........37, 183, 231

Congenital defect........74, 76

CONLEY, H. P.......... 283

Constipation, chapter on......

$\ldots \ldots .77,38,178,221,235,313$

-causes ............ 80

-diagnosis ............ 83

-treatment ............. 84

-rectal nuassage.......... 86

Corrugator Cutis Ani........ 18

CORSONS, E. A........... 282

COUNCILMAN and LE FLUER

$\ldots \ldots \ldots 239,248,258,263$

CRAIG ............... 259

CRIPPS, HARRISON . . 28, 110, 169

CRISLER, J. A.......... 283

Cropology (see Feces, exam-

anination of) $\ldots \ldots \ldots \ldots 326$

Cryptitis, chapter on.....209, 218

-symptoms .............. 218

-treatment ............ 219

Crypts of Lieberkuhn....... 235

Crypts of Morgani....18, 20.

$124,147,168,189,209,213,307$

D.

Defecation, disturbances of (see abscess, constipation, fissule, hemorrhoids, impaction, rectal valve).

—physiology of....... 78, 326

DE VILBISS' Speculum...145, 167

- spray tube ............ 227

Diarrhoea ..............

$9 S, 166,222,231,244,316,327$ Diet.

-in constipation........... 81

-in dysentery.............266 
Diet, in fissure.......... 127

-in hemorrhoids........... 208

-test .............327. 381

Digestion, disturbance of .... 40

Digital examination......48, 135

Dilatation of sphincters....... $\ldots \ldots 54,104,130,186,193,199$

-local anesthesia for....... 299

Discharge .........38, 98

$113,114,154,166,168,209$

$222,235,259,260,314,332,342$

Douglas, pouch of........ 28

Dysentery, chapter on...... 238

-distribution ............ 239

-general etiology......... 239

-history ............... 238

-acute catarrhal.......... 243

- diagnosis ............. 245

-pathology ............ 243

-prognosis ............ 245

-special etiology ........ 243

-symptoms .............. 244

-amoebic .............. 248

-case reports.........260, 261

-complications .......... 263

--diagnosis .............264

-etiology ............. 248

-pathology ............ 253

-prognosis ............ 264

-sequelae .............. 263

-treatment ........... 265

- diet ................ 266

-intestinal antiseptics...... 270

-irrigations ........... 274

-laxatives .............. 269

-prophylaxis ........... 265

--remedial .............. 267

-chronic amoebic .......... 279

-diphtheritic ........... 245

- complications ......... 247

- -diagnosis ............. 247

-etiology .............. 246

-pathology $\ldots \ldots \ldots \ldots \ldots 246$

-symptoms ........... 246
Dysentery, treatment......247

-secondary diphtheritic...... 247

-prognosis ............ 248

--symptoms ............ 248

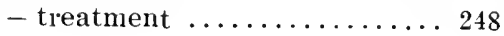

E.

Elevations $\ldots \ldots \ldots \ldots \ldots \ldots .37$

Enema ...........61, 111

Entamoeba Histolytica.......

$.248,250,252$

Eucain (see Lccal Anesthesia) $\ldots \ldots \ldots \ldots \ldots 114,115,296$

Eversion of anus......54, 55, 56

Exaggerated lithoton!y position $\ldots \ldots \ldots \ldots \ldots \ldots \ldots \ldots 2,186$

Exaggerated Sims' position.... 281

Examination, Chapter on ...41, 313

-abdomino-rectal (see Rectoabdominal) .......... 57

-abdomino-vaginal ........ 58

-anoscopic (see Anoscopy)... 64

-digital .............49, 52

-of feces (see Feces)...... 326

-protoscopic (see Proctoscopy) ............. 67

-sigmoidoscopic (see Sigmoidoscopy ) ........... 73

-vagino-rectal .......... 54

External Sphincter (see Sphincter Ani External)....... 18

\section{F.}

Fecal impaction ........... 97

Feces (see Stools, defecation) 326

- character of in intestinal affections ............. 361

-acute intestinal catarrh (see Proctitis) ........... 361

-amoebic dysentery (see Dysentery) $\ldots \ldots \ldots \ldots \ldots \ldots 362$

- cholera nostras.......... 362

- chronic intestinal catarrh (see Proctitis) ........ 362

—diphtheritic enteritis...... 362 
Feces (see Stools, Defecation).

- dysentery (see chapter on disentery ) ............ 362

-muco-membranous colitis

(see sigmoiditis)........ 362

-chemical examination of..... 336

-fermentation test......... 336

-sublimate test.......... 336

-clinical examination of,

Chapter on.........326, 331

-macroscopic elements..... 329

-misroscopic elements....... 330

- examination ............. 333

-normal.......326, 327, 328, 329

-pathologic elements....... 332

- clinical significance of tests. 339

-concretions in............ 346

-estimation of lost albumen.. 338

-gallstones in.............345

-location of.............. 53

- parasites in (see Parasites). 346

-tests for blood in......... 344

-Benzidine test............. 344

-Holland's test............. 344

-Klunge's test............. 344

-Webber's test............. 343

Finger cot............. 53

Fissure in ano, Chapter on....

$122,187,209,219,235,306,314$

-after care.............. 132

-diagnosis ............. 126

-etiology ................ 123

--symptoms ............... 126

-treatment ............. 126

- surgical $\ldots \ldots \ldots \ldots \ldots \ldots 130$

- excision .............138, 134

-author's operation......133, 134

-incision ............... 130

-multiple ............... 123

Fistula in ano, Chapter on... $.142,146,152,306,323$

-classification ............. 153

- etiology ................ 152

-blind external ............ 164
Fistula in ano, diagnosis...... 166

—symptoms ............... 166

-treatment .............. 166

-blind internal............ 166

—diagnosis ............... 167

-symptoms .............. 166

-treatment .............. 167

-horse shoe.............. 153

-multiple ............... 153

- simple complete.......... 154

-after care............... 162

- diagnosis ................ 154

-symptoms .............. 154

-treatment .............. 158

-excision ................. 159

-author's operation........ 160

-incision ................ 159

-injection of bismuth paste. 160

-ligature operations........ 162

-sub-muco-cutaneous ....... 169

-sub-mucous ..........168, 169

-tuberculous ............. 171

- diagnosis ............... 171

- symptoms .............. 171

-treatment ............. 172

Folds, Houston's (see Rectal valves) $\ldots \ldots \ldots \ldots \ldots \ldots, 22$

Forceps, alligator.......... 67

Foreign body in rectum....40,329

-local anesthesia for removal of ..................308

Formalin-Boric solution...... 273

Formulae .......90, 108, 109, $110,111,129,132,191$, $194,200,201,271,273,274,277$

FRANCK .............. 192

G.

Gallstones (see Impactions, foreign bodies .......... 345

GANT, S. G........29, 92, 111

$\mathrm{H}$.

HAMILTON, E. A........ 114 HARRIS, H. F...239, 256, 275, 282 
Hemorrhage (see Bleeding).

Hemorrhoidal arteries (see Arteries-frontispiece) ...... 29,30 ,

-forceps, author's ........ 196

-nerves (see Nerves)......30, 32

-veins (see Veins - frontispiece) ................

Hemorrhoids, Chapter on.....

$173,35,37,84,209,236,288$,

..............304, 318, 323

- classification ........... 175

-external ................ 175

-integumentary .......175, 178

-treatment .............206

-thrombotic .......175, 176, 183

-treatment ..........205, 305

-varicose ............. 176

-internal ............176, 183

- -general etiology ......... 177

-capillary ............. 176

-granular .............. 176

-varicose ...........176, 177

- diagnosis .............. 184

-etiology ............. 177

-symptoms ............. 180

-treatment ............ 190

-cautery .............. 191

-excision .............. 195

-author's operation...197, 199, 201

-injcction ............ 191

-palliative ............. 190

-sub-mucous excision....... 203

-surgical general ......... 193

-interno-external.179, 180, 182, 198

-treatment ............. 198

HILTON, white line of...... 22

HIRSCH, A. ........... 239

HIRSCHMAN, L. J.

-aroscopes...662, 65, 66, 157, 168

-bloodless operation for hem-

orrhoids ......197, 199, 201

-blunt ligature carrier....... 198
HIRSCHMAN, L. J.

-dilating rectal massage bag .............86, 87

-hemorrhoidal forceps....... 196

-method of rectal massage... 85

-modification of Ball's operation 117

-operation for exision of fistula .............. 160

-for excision of fissure....133, 134

- for rectal valvotomy..92, 94, 95 -proctoscopes ......70,92,308

-rectal retractor.......... 197

-rectal scissors ........94, 168

-rectal spray tube......... 225

-sigmoidoscope .......... 73

—valvotomy needle........ 92

HOLL ................ 27

Holland's test ............ 342

HOUSTON (see Rectal valves) 22

-folds of (see Rectal valves) 22

-valves of (see Rectal valves) 22

I

Ichthyol ..................

$112,128,129,228,232,234,279$

Ilio-coccygeus (see Levator

ani ............... 26

Impaction fecal, Chapter on.97, 221

-causes ............. 97

- diagnosis ............ 99

-symptoms ............ 98

-treatment ............ 99

Indigestion $\ldots \ldots \ldots \ldots 222,231,259$

Inspection, External ...... 48

-internal (see Anoscopy, Proctoscopy, Sigmoidoscopy) 61,64

Instruments-

-for anoscopy.......... 64

-inspection ............. 100

-local anesthesia.......... 298

-office treatment of dysen-

tery ............... 272

-proctoscopy .......... 68

- sigmoidoscopy .......... 73 
Instruments-

-surgical treatment of fissure ........127, 133, 134 fistula $\ldots \ldots 158,160,166,167$ hemorrhoids .......191, 199 pruritus .......114, 115, 117 Intermural abscess (see $\mathrm{Ab}$ scess ............... 142

Internal Sphincter (see Sphincter ani, internal)........ 22

lodide of mercury........... 48 Ischio-rectal abscess (see Abscess) ............60, 146

-fossa ................ 28

Itching (see Pruritus ani..... $\ldots \ldots \ldots \ldots 36,107,154,166$ $\mathrm{J}$

JELKS, J. L...........2227, 238 -rectal tube.......227, 232, 274

\section{$\mathrm{K}$}

IJARTULIS ......2239, 271, 272 KELLY, Anoscope ......65, 67

-leg holder ............ 5

-sigmoidoscope .......... 73

KELSEY, CHARLES.....110, 191

Klunge's test ............ 344

Knee-elbow position......... 63

knee-shoulder position........ $\ldots \ldots \ldots \ldots 63,67,186,278$

KRAMERIA, extract of...226, 232 KRAUSS, WM. .......... 261

I.ancet-Clinic $\ldots \ldots \ldots \ldots \ldots \ldots, 169$

Lateral Ligaments.......... 27

Lateral position (see Sims' position) ............. 51

LE ROY, LOUIS........... 279

Lesser Sphincterian Nerve... 20

levator-ani muscle.......... 24

I.IEBERKAUHN $\ldots \ldots \ldots 235,246$

Ligaments of rectum....... 27 Ligature carrier, blunt pointed, Author"s
Ligature operation for Fistu'a (see Fistula) ......... 162

Ligature, rubber (see Valvotomy, Fistula, Stricture)... 322

Light, Electric head........ 43 Jinea-dentata ...........18, 20

Lithotomy position........... $\ldots \ldots .55,56,58,100,144,148$

Local anesthesia, Chapter on $295,20,54,100,114,117$, $130,133,140,143,144,145$, $149,158,159,160,167,193,207$ -amount of distension necessary for ................301

- -anesthetics used.......... 296

--apparatus necessary........ 298

-limitation of, Chapter on... 312

--point of puncture for.......300

-position of patient for..... 299

- preparation of patient for...29y

-technique for dilatation of sphincters under ........ 303

-operating for acute thrombotic hemorrhoids ....... 305 -operating for external hemorrhoids ............. 304

-operating for fissure in ano. . 306 -operating for fistula in ano. . 306 -operating for hypertrophy of anal papillae ........... §07 -operating for hypertrophy of rectal valves .......... 308

-operating for internal hemorrhoids .............. 19.3

-operating for peri-anal abscesses ............... 305

-operating for posterior proctotomy .............. 310

-operating for removal foreign bodies ................ 308

-operating for removal perianal growth .......... 309 Lubricant ...........50, 277 
Lymphatic glands :

-inguinal ............. 21

- lumbar ............... 32

-pre-sacral or post-rectal.... 32

Lymphatic vessels.......... 31

\section{M}

MAC MILLAN, J. A........

Malformations of anus, Congenita! ...............74, 76

MARTIN, T. C......22, 69, 92

Marginal abscess (see Abscess, sub-tegumentary) ....... 140

Massage, Rectal, Author's method of $\ldots \ldots \ldots \ldots .85,101$

MAUREL .............. 327

McGREGOR. . ............ 239

Menstruation, Disturbances of 39

Methylene blue........... 64

MEYER, W. ............ 282

Middle hemorrhoidal vessels.. 27

Milk of Bismuth.......... 64

Milk of Magnesia.......... 64

MORESTIN ............ 32

MURGaGNI, Columns of..... 23

-crypts of..........1s, 20. 124

MUSGRAVE....239, 258, 263, 275

\section{$\mathbf{N}$}

Nausea .............. 40

Nerve, fifth sacral.......20, 32

-fourth sacral .......21, 32, 116

-internal pudic ........... 20

-lesser sphincterian.......20, 32

-anesthetization of ....... 302

- sixth sacral ..........20, 32

— sympathetic ........... 32

-third sacral .......20, 32, 116

Nitrous oxide....100, 130, 291, 322

Non-surgical treatment of fissure .............. 127

-fistula .............. 162

-hemorrhoids ........... 190

-proctitis and sigmoiditis 223, 231
Non-surgical treatment of fissure, ulcer ........... 162

Nux-vomica $\ldots \ldots \ldots \ldots \ldots \ldots \quad \delta 9$

\section{o}

Obstipation (see rectal valve, constipation), Chapter on.. $\ldots \ldots \ldots \ldots 7,90,98,38$

-etiology ............ 90

-treatment ..........95, 93

Office treatment of rectal diseases, Limitations of..... 312

Oil, White petroleum (see Albolene) ........... 89

Operating room equipment.... 41

OSLER, IVILLIAM ...239, 248, 275

Oxyuris vermicularis (see parasites, pin worms)....103, 350

Pain..33, 39, 126, 141, 143, 147, $154,182,235,244259$

Pal pation, bimanual.......60, 148

-recto-abdominal ........57, 99

Pancreatin ........89, 232, 236

Papilla, Anal, Chapter on... $\ldots \ldots 209,20,189,211,214,307$

-hypertrophy of ......... 211

- diagnosis ............. 214

-examination ............ 214

-symptoms ............. 217

-treatment ........219, 220, 307

Parasites ..........103, 221, 248

-animal in feces......... 346

-cestodes ............... 357

-bothriocephalus latus ..... 360

-taenia nana............ 360

-taenia saginatta .......358, 359

-taenia solium........... 348

-nematodes .............. 350

-anchlyostoma duodenale.... 352

-anguillula intestinalis....... 356

-ascaris lumbricoides.....108, 349 
Parasites, oxyuris vermiculars

(see pin worms) ......350,351

- trichina spiralis ......... 355

-trichocephalus dispar...... 354

-uncinaria Americana....... 353

-protoroa ............347, 346

-amoeba (see Dystentery).... 346

-balantidum coli.......347, 348

PENNINGTON, J. R...92, 169, 170

Peri-anal abscess (see Abscess) 137

Peristalsis ............24, 78

Peroxide of hydrogen.......

$\ldots \ldots \ldots 64,100,109,157,282$

Physiology of defecation....78, 326

Piles (see Hemorrhoids)..... 173

Pin worms (see oxyuris vermi-

cularis) $\ldots \ldots 40,103,109,351$

Plicae transversalis recti (see rectal valves) .......... 22

Polypus, Chapter on...125, 188, 288

-classification ........... 209

- diagnosis ............. 210

-symptoms ............. 209

-treatment ............ 210

Position, Exaggerated lithot-

omy ...........72, 186

- -exaggerated Sims' ......... 281

-knee-shoulder .....63, 67, 186, 278

-lateral (see Sims'......... 48

-lithotomy (see Lithotomy).. 55

-rectal massage .......... 88

-squatting ...........59, 61

Posture, Characteristic in rec-

tal disease ........... 141

-sitting, characteristic...... 45

Probe .......6.65, 66, 158, 167

Proctitis, Chapter on..221, 187, 167

-acute ............... 221

diagnosis ............ 223

etiology ............. 221

symptoms ............ 222

treatment ........... 223

-chronic .............. 229
Proctitis, atrophic ......... 234

pathology ............ 234

symptoms ........... 235

treatment ............ 236

-general etiology ......... 229

-hypertrophic .......... 230

diagnosis ........... 231

pathology ........... 230

symptoms ........... $230^{\circ}$

treatment ............2231

Proctoscope ........68, 92, 308

Proctoscopy (see knee-shoulder position) ...67, 69, 181, 186, 278

—without instruments .....68, 99

Proctotomy, Local anesthesia..

for ............... 310

Prolapse of anus......... 284

Prolapse of rectum.........

$\ldots \ldots \ldots \ldots 35,189,209,222,324$

-classification ........... 284

-concealed ...........286, 298

-in children, Chapter on.... 284

diagnosis ............ 287

etiology .........286, 287

prophylaxis ..........291

symptoms ............ 297

treatment ............288

actual cautery ........ 293

cauterization with nitric acid .........291, 292

Prolapsing internal hemorrhoids (see Hemorrhoids).. 184

Protrusions ........37, 188, 189

Pruritus ani (see Itching), Chapter on .............. $102,126,165,183,218,231,235$

-appearance of parts in...105, 106

- characteristics of ........ 107

- -etiology ............... 102

-treatment ............ 108

Ball's operation ....... 116 general surgical treatment 114 Hamilton's operation..... 114

Pubo-coccygeus (see Levator ani) $\ldots \ldots \ldots \ldots \ldots \ldots \ldots$ 
Pubo-rectalis (see Levator ani-

Pus (see Abscess, Discharge, Fistula, Proctitis)..38, 98, 342

\section{Q}

Quadrants of anus........ 46

\section{$\mathbf{R}$}

Record card (Author's).... 47, 48

Rectal (see Rectum):

- chambers 22

-dressing

-retractor (Author's modified) 197

-scissors, Author's angular... 94

-spray ............... 100

— spray tube, Author's....... 225

-stricture (see Stricture, Rectal) $\ldots \ldots \ldots \ldots .310,321,322$ -tube .....100, 227, 232, 238, 274

—ulcer (see Ulcer, r'ectal) ..... 98

-valves ...........22, 23, $69,71,90,91,223,277,308,322$ -valvotomy (see Valvotomy).. 92

Recto-abdominal palpation.... 57

Recto-Vesical pouch ......... 28

Rectum (see rectal) anatomy of 20 -prolapse of (see Prolapse).. 284 -relations of ............. 28

- ulcer of (see Ulcer)........ 98

Restlessness ............ 40

Ring worm ...........103, 109

Rubber ligature (see Fistula,

Proctotomy, Valvotomy).. 322

\section{s}

Sacral backache...39, 147, 222, 235

Scabies ..............103, 109

SCHMJDT. ............. 331

Scissor's, Author's angular...94, 168

-sharp, curved .......... 114

Scybala ............231, 245

Sentinel pile (see Fissure) .124. 126 SHIGA ............239, 246

Sigmoid colon (see Chapter

XII ) $\ldots \ldots \ldots \ldots \ldots 22, \quad 28$
Sigmoiditis $\ldots \ldots \ldots \ldots \ldots \ldots .221$

-acute ................. 221

etiology ............ 221

symptoms ............ 222

treatment ........... 223

-chronic ............... 229

general etiology ........ 229

symptoms and treatment

see Proctitis, Chronic) 230, 231

Sigmoidoscope ........... 73

Sigmoidoscopy (see Exaggerat-

ed Lithotomy position)....

$73,99,186$

silver, Nitrate of..112, 129, 234, 279

sims' position.....48, 51, 87,

$100,117,142,144,148,185,276$

Spasm ...........34, 142, 255

Speculum:

-author's fenestrated (see anoscope) ..........62, 65

—bivalve, rectal ......... 68

DE VILBISS ...........145, 167

Sphincter Ani External (see dilatation) $\ldots \ldots 18,124,150,216$

-local anesthesia for dilatation of $\ldots \ldots \ldots \ldots \ldots \ldots \ldots 299$

—spasmodic contraction of... 142

Sphincter ani internal....... 22

Sphincter recti (Pubo-rectalis) 26

Squatting position .......59, 61

Steele's Fermentation appar-

atus .................. 336

Sterile Water anesthesia..... 297

Sterilizers, instrument....... 44

STERNBERG ............ 239

STILES.............. 353

Stools (see Feces) ......... 326

-altered .............39, 361

STRASBURGER ......... 336

STRAUSS ............. 327

Stricture, Rectal.....310, 321, 322

STRONG ...239, 246, 258, 263, 275

Subcutaneous abscess (see Ab-

scess) .........140, 154, 165 
Submuco-cutaneous a b s ces s (see Abscess) 142, 154, 166, 167 Submucous abscess (see Abscess) $\ldots \ldots 142,154,166,167$ Submucous tract (see Fistula.

Submucous) ........... 167

Subtegumentary abscess (see

Abscess) ......140, 154, 165

Suppository (see Formulae)..

$\ldots \ldots \ldots 130,159,194,271,274$

Symbiosis, Bacteria of (see

Dysentery) ....251, 252, 255

Symptoms calling for rectal examination (Chapter on).. 33

\section{$\mathbf{T}$}

Table, Operating .......... 42

Taenia nana (see Parasites).. 360

-saginatia (see Parasites)... 358

--solium (see Parasites)..... 358

Taka Diastase............ 89

'Tampton, Rectal .......... 85

TEACHNOR, W. .......... 85

Teohnique of examination of anus and rectum, Chapter 41

- of use of local anesthesia,

Chapter on............. 295

Tenderness ..34, 140, 141, 143, 148

Tenesmus..9\$, 235,244, 247, 259, 316

THEVENOL ............ 243

THONLPSON ..........26, 27

Tract, Submucous see (Fistula, Submucous) …...... 167

Trichina Spiralis (see Parasites) ................ 355

Trichocephalus Dispar (see Parasites) .............. 354

Tuberculous fistulae (see Fistula ) ............. 171

'URCK, F. B............ 85

TUTTLE, J. P.......74, 110, $137,226,230,232,234,239,282$
$\mathrm{U}$

Jlcer, Anal, Chapter on....... $122,112,124,135,136,236,314$ -excision of $\ldots \ldots \ldots \ldots \ldots 135,136$

Ulcer, rectal.............. ... 98, 187, 233, 236, 244, 321 - Amoebic ...........253, 255

Uncinaria Americana (see Anchylostoma Duodenale).... 353

Urination, Frequent painful, Disturbances of......... $40,144,147,222$

Uterine disease......40, 98, 103

\section{V}

Vagina, discharge from.......103

Vaginal rectal examination.... 54

Valves, Semi-lunar (see Crypts of Morgagni)..........208

-rectal (see Rectal Valves).. 22 Valvotomy, rectal........90, 283 -Author's operation for...... 93 -local anesthesia for........ 308

-needle ................. 92

Veins (see Frontispicce)

--inferior hemorrhoidal....... 31

-internal iliac ............. 31

-middle hemorrhoidal........ 31

-superior hemorrhoidal........ 31

Vibrator, mechanical..112, 193, 299

W

WAGNER, G. W......... 326 Wales Bougie......69, 86, 310, 321 WALLIS, F. C......112, 168, 219 iVarts, Venereal........... 189 Wasserman test for syphilis... 236 Webber's test............ 343 WEIR, R. . ............. 282

Whitehead operation ....... 204 WOODWARD......... 238, 240 Worms, Intestinal (see Parasites) ........40, 103, 348 


\title{
Diagnosis and Treatment of Diseases of Women
}

\author{
By \\ H. S. Crossen, M. D.
}

Clinical Professor of Gynecology, Medical Department Washington University; Gynecologist to the Washington University Hospital, and Chief of the Gynecological Clinic; Consulting Gynecologist to the Bethesda Hospital, St. Louis Female Hospital, and St. Lounis City Hospital.

816 Pages. 700 Illustrations.

Price: Cloth \$6.00. One-half Morocco \$7.50 Sent anywhere prepaid, upon receipt of price.

\section{CONTENTS.}

Chapter I. Gynecologic Examination Methods.

Chapter

Chapter

Chapter

Chapter

II. Gynecologic Diagnosis.

III. Gynecologic Treatment.

IV. Diseases of External Genitals and Vagina.

V. Lacerations and Fistula of Pelvic Floor, Perineum, External Genitals and Vagina.

Chapter

Chapter

Chapter

Chapter

Chapter

Chapter

Chapter

Chapter

Chapter

Chapter

Chapter

Chapter

VI. Inflammatory and Nutritive Diseases of the Uterus.

VII. Displacements of the Uterus.

VIII. Fibromyoma of the Uterus.

IX. Malignant Disease of the Uterus.

X. Pelvic Inflammation.

XI. Other Affections of Fallopian Tubes, Peritoneum and Connective Tissue.

XII. Diseases of the Ovary and Parovarium.

XIII. Malformations.

XIV. Disturbance of Functions.

XV. Invasion of the Peritoneal Cavity for the Treatment of Gynecological Diseases.

XVI. After-Treatment of Operative Cases.

XVII. Medico-Legal Points in Gynecology.

Appendix-Formula-Index.

\section{V. Mosby Medical Book and Publishing Co. \\ Grand Ave. and Olive Street, St. Louis, Mo.}




\section{Golden Rules of Pediatrics}

Aphorisms, Observations and Precepts on the Science and Art of Pediatrics: Giving the Practical Rules for Diagnosis and Prognosis, the Essentials of Infant

Feeding, and the Principles of Scientific Treatment.

BY

JOHN ZAHORSKY, A. B., M. D.

Clinical Professor of Pediatrics, Washington University Medical Department, St. Louis;

Ex-President Bethesda Society; Attending Physician to the Bethesda Found-

lings' Home; Member of the American Medical Association and of the

St. Louis Academy of Science; Editor of the St. Louis Courier

of Medicine; Author of "Baby Incubators," etc.

WITH AN INTRODUCTION

BY

E. W. SAUNDERS, M. D.

Professor of Diseases of Children and Clinical Midwifery, Washington University, St. Louis, Mo.

370 Pages, Silk Cloth Binding, Price $\$ 3.00$ Sent anywhere prepaid, upon receipt of price.

\section{CONTENTS:}

Introduction.

Part 1.

General Rules of Diagnosis.

General Rules.

Loss in Weight. Appetite.

Convulsions.

Physical Examination.

Head and Neck.

Some Deformities.

Teeth and Gums. Dentrition.

The Enanthemata.

Vomiting. Hematemesis.

Diarrhea.

Distended Abdomen.

Abdoninal Pain.

Abdominal Swellings.

The Nose and Nasopharynx.

The Larynx.

Anomalies of Breathing. Cough.

The Lungs.

The Heart and Circulation.

The Urine. The Eruptions.

The Nervous System.

Paralysis.

'Tremor, Choreiform Movements. Headache, etc.

Changes About the Eyes.

Changes About the Ear.

Clinical Syndromes.

Fever. Chronic Fever.

Status Gastricus.

The Typhoid State.

Infantile Atrophy.

Gastroenteric Infection, Diarrhea.

Chronic Indigestion in Older Children.

Chronic Constipation.

Peritoneal Irritation.

Severe Anemia. Edena.
Ascites. The Adenoid Face.

Acute Pneumonic Consolidation.

Intestiual Obstruction.

Nervous State. Scrofula.

Tuberculosis.

Impending Heart Failure.

The Syndrome of Cerebral Irritations.

\section{Golden Rules of Prognosis.}

Part 11 .

Golden Rules of Hygiene and Infant Feeding.

The Nursing Mother.

The Wet Nurse.

Artificial Feeding.

Feeding the Sick.

Golden Rules of Treatment.

General Therapeutics.

The Newly Born.

Diseases of the Mouth.

The Neck and Scalp.

The Throat.

The Respiratory Organs.

Gastroenteric Diseases.

Rickets and Scurvy.

Heart and Circulation.

The Blood.

The Genito-Urinary Organs.

The Nervous System.

Specific Infectious Diseases.

Malaria. Cerebro-Spinal Fever.

Diphtheria. Intubation.

Tuberculosis. Pertussis.

Mumps. Septicemia.

Rheumatism and Endocarditis.

Syphilis. The Exanthemata.

The Severe Infectious Fevers.

The Skin.

Formulary.

C. V. Mosby Medical Book and Publishing Co'. 


\section{Golden Rules of Surgery}

Aphorisms Observations Reflections

\section{On the \\ Science and Art of Surgery}

A Guide for Surgeons and Those Who Would Become Surgeons

By

\section{Augustus Charles Bernays, A. M., M. D. \\ F. R. C. S., England}

Late Chief Surgeon Lutheran Hospital and for Twenty Years Professor of Surgery and Anatomy, St. Louis, Mo., U. S. A.

230 Pages. Handsome Cloth Binding. Price, \$2.50.

Sent Anywhere, Prepaid, Upon Receipt of Price.

\section{CONTENTS:}

The Education of a Surgeon.

On Scientific Communications to the

Literature of Medicine and Surgery.

Science and Surgery.
About Fees.

Off With the Cloak of Superstition.

Inflammation and the Confusion It Has Caused.

GOLDEN RULES OF SURGERY:

Asepsis.

Anesthesia.

Abscesses.

Abdomen.

Appendicitis.

Aneurysm.

Artery Bleeding.

Burns.

Breast.
Genito-Urinary.

Operations.

Joints.

Ear.

Erysipelas.

Gangrene.

Hand and Foot.

Moist Dressing.

Mouth.
Nose.

Goitre.

Shock.

Oesophagus.

Pelvis.

Rectum.

Spine.

Throat.

Veins.

Can Minor Surgical Operations Be Done in Office?

Death Following Minor Surgical Operations.

Fractures and Dislocations.

Irrigation Drainage of Abdominal Cavity.

Minor Surgical Operations. Stomach and Intestines.

C. V. Mosby Medical Book and Publishing Co.

Grand Ave. and Olive Street., St. Louis, Mo. 


\section{Golden Rules of Dietetics}

By A. L. BENEDICT, A. M., M. D.

Consultant in Digestive Diseases. City and Riverside Hospitals and Attendant in same; Nercy Hospital, Buffalo; Member of the Academy of Medicine and of American Gastro-Entrological Association, etc.;

Author of Practical Dietetics.

\section{CONTENTS.}

\section{Part I.}

Chapter

Chapter

Chapter

Chapter

Chapter

Chapter

Chapter

Chapter

Chapter

Chapter

Cliapter

Chapter

Chapter

Chapter

Chapter

Chapter

Chapter

Chapter

Chapter

Chapter

Chapter

Chapter

Chapter

Chapter

Chapter

Chapter

Cliapter

Chapter

Chapter

Chapter

Chapter

Chapter

Chapter

Chapter

Chapter

Chapter

Chapter

Chapter

Chapter

Chapter

Chapter

Chapter XXIII.

Chapter XXIV.

XVI

XIX.

XX.
I. Phrsiologic Chemistry.

II. Daily Requirements of the Human Body.

III. Standard Diet in Health.

IV. Quantative Estimate of Diet.

V. Approximate Methods of Checking Diet Weight and the Excretions.

VI. Transwnutability and Reservation of Food.

VII. Waste of Food.

VIII. Predigestion of Food.

IX. Emergency Methods of Introducing Nourishment.

$\mathrm{X}$. Preserved Foods.

XI. Methods of Cooking.

XII. Compositions of Natural and Commercial Foodstuffs.

XIlI. Food Adjuncts.

XIV. Purine Bodies.

XV. Important Constituents of Foodstuffs.

Distinctly Deleterious Foodstuffs.

General Hygiene of Eating.

Diet 'Tests.

Condensation of Atwater \& Bryant's Analysis of Foodstuffs.

\section{Part II.}

I. Principles of Dietetics According to General Pathologic Conditions.

II. Infant Feeding.

III. Diet in Critical Physiologic Periods.

IV. Diabetis, Glycosuria.

V. Obesity and Leanness.

VI. Chronic Diseases of Nitrogeneous Metabolism.

VII. Diseases of the Urinary Organs.

VIII. Diseases of the Ductless Glinds.

IX. Diseases of the Liver.

$\mathrm{X}$. Diseases of the Pancreas.

XI. Diseases of the Digestive Organs.

XII. General Preversion of Digestive Functions.

XIII. Functional Intestinal Diseases.

XIV. Organic Intestinal Diseases.

XV. Diseases of the Heart and Blood Vessels.

XVI. Blood Diseases.

XVII. Hemorrhagic Diseases.

III. Bone Diseases.

General Principles of Feeding in Fevers.

Infectious and Parasite Diseases.

Respiratory Diseases.

Skin Diseases.

Diseases of the Nervous Sustem.

Surgical Emergencies and Operations.

450 Pages, Octavo. Price $\$ 3.00$

C. V. Mosby Medical Book and Publishing Co.

Grand Avenue and Olive Street, St: Louis, Mo. 


\section{A VERY}

\section{YOUNG OVUM IN SITU}

\section{By \\ G. Leopold,}

Dresden

Authorized Translation By

W. H. VOGT, A. M., M. D.

Obstetrician and Gynecologist, Lutheran Hospital, St. Louis, Mo.

65 Pages Text. 35 Pages Lithographic Illustrations in Colors Price, Gloth $\$ 3.50$

\section{Publisher's Announcement}

The importance of an understanding of embryology is becoming nore and more apparent. The student now realizes that anatomy is much better understood-where it is worked out from the standpoint of embryonic development-than where it is learned in its crude state in the dissecting-room. The surgeon realizes that he can better grasp the relationship of structures when he is familiar with their formation from the embryo. The scheme of development as worked out by Leopold represents the latest work along this line. The work is most scientific and cannot fail to interest all who are seeking the fundamental truths of embryonic development.

\section{THE C. V. MOSBY MEDICAL BOOK AND PUBLISHING CO.}




\section{Examination of The Ear}

By

SELDEN SPENCER, A. B., M. D.

Instructor of Otology in the Washington University Medical Department, St. Louis, Mo.

With an Introduction

By

H. N. SPENCER, M. D., LL. D.

Professor of Otology, Medical Department Washington University, St. Louis, Mo.

\section{PAGES OF TEXT \\ 5 FULL PAGE PLATES \\ 12 OTHER ILLUSTRATIONS \\ PRICE $\$ 1.00$}

\section{CONTENTS:}

Chapter I. Methods of Procedure (General Consideration).

Chapter

II. The External Ear.

Chapter III. Diseases of the Canal.

Chapter IV. The Middle Ear.

Chapter V. The Middle Ear (Continued), Non-Suppurative Conditions.

Chapter VI. The Middle Ear (Continued), Post-Suppurative Conditions.

Chapter VII. The Middle Ear (Continued), Suppurative Conditions.

Chapter VIII. The Mliddle Ear (Continued), Purulent Otitis Media.

Chapter XI. The Middle Ear (Continued), Purulent Otitis Media.

Chapter X. The Middle Ear (Continued), Operations in Chronic Purulent Otitis Media.

Chapter XI. The Internal Ear.

Chapter XII. Hearing Tests.

Chapter XIII. Ir:tra-Cranial Complications.

Chapter XIV. Exercises in the Surgical Anatomy of the Temporal Bone.

\section{THE C. V. MOSBY MEDICAL BOOK AND PUBLISHING CO.}

Grand Ave, and Olive Street., St. Louis, Mo. 


\title{
Suggestive Therapeutics, Applied Hypnotism and Psychic Science
}

\author{
By H. S. MUNRO, A. M., M. D. \\ Americus, $\mathrm{Ga}$.
}

376 Pages, Octavo. Price, 3.00. Sent anywhere upon receipt of price.

\section{Publisher's Announcement.}

That stiggestion is an important factor in the treatment of diseases is no longer denied by those that keep abreast of medical progress. The medical journals are replete with articles from the leading alienists and internists of this country. The profession in Europe has been alive to the importance of this subject for many years and much has been written on it by such men as Schofield, Bernheim, Forell and Duboise. These writings have been in the nature of research work and have not been devoted to the practical application of this branch of therapeutics in everyday practice. The book herein described is designed to give a practical working guide to the general practitioner in the application of suggestive therapeutics. Its purpose is to aid the profession in reaching a correct understanding of a subject that has been shrouded in mystery and used by the quack and charlatan in many cases to discredit scientific medicine. The indorsements that have been given the author by prominent physicians and surgeons are the best recommendations the book can have.

CONTENTS:

Chapter

Chapter

Chapter

Chapter

Chapter

Chapter

Chapter

Chapter

Chapter

Chapter

Chapter

Chapter

Chapter

Cliapter

Chapter

Chapter

Chapter

Cliapter XVIII.

Chapter XIX.

Chapter XX.

I. Introduction.

\section{Suggestion: Its Uses and Abuses.}

III. Hypnotism: A Demonstration of the Efficiency of Suggestion.

Technique of Inducing the Hypnotic State.

IV. Theory and Practice of Suggestive Therapeutics.

V. Simple Suggestions, or Suggestion Without Hypnotism.

VI. Hypnotic Suggestive Therapeutics Applied in Medicine, Surgery.

VII. The Psychological Factor in Obstetrics.

VIII. Training the Subconscious Self for Health and Strength.

IX. Correct Diagnosis a Safeguard Against Blunders.

$\mathrm{X}$. Philosophy and Religion and Their Relation to Health.

XI. Conservation of Energy, Education and Control of Emotions.

Breathing, Relaxation. Dietetics, Exercise, etc.

XII. Roughing It as a Means of Health.

XIII. Are All Specialists Egotists?

XIV. Pensonality as a Factor in Therapentics.

XV. Environment: Its Influence in Therapeutics.

XVI. Brutality of Frankness: Honesty Imperative.

XVII. Physical and Mental Hygiene; Character as a Resource of Health.

Suggestion in Education, Character Building, etc.

Moral Stamina a Therapeutic Power; The Higher Art in Therapeutics, and the True Physician.

\section{V. Mosby Medical Book and Publishing Co.}

Grand Ave. and Olive Street., St. Louis, Mo. 


\title{
Office Treatment of Rectal Diseases
}

\author{
By \\ R. D. Mason, M. D.
}

Professor of Rectal Diseases in the Creighton University, Omaha, Nebraska.

New 4th Edition. 367 Pages. $\quad 87$ Illustrations. Price $\$ 2.50$.

\section{Tuberculosis of the Nose and Throat}

By

Lorenzo B. Lockord, A. B., M. D.

Denver, Colorado

Consulting Laryngologist to the Agnes Memorial Hospital, Denver.

504 Pages. 85 lllustrations, 64 of which are colored. Price, $\$ 5.00$

C. V. Mosby Medical Book and Publishing Co. Grand Ave. and Olive Street., St. Louis, Mo. 


\section{Arteriosclerosis}

\section{ETIOLOGY,}

150 PAGES.
DIAGNOSIS, PROGNOSIS, AND TREATMENT

By

L. M. Warfield, A. M., M. D.

With an Introduction by

H. S. Thayer, Baltimore, Md.

8 ORIGINAL ILLUSTRATIONS.

\section{PUBLISHERS ANNOUNCEMENT}

This book is particularly opportune. The rapid pace which Americans are living, the worry and mental strain under which the majority of their time is spent, has made this a nation of arterio-sclerotics. The author has laid stress "pon prophylaxis as well as given the most rational treatment known to modern times. The text is embellished with instructive original illustrations. Sent anywhere on receipt of price.

\section{Diseases of the Skin \\ By}

A. H. Ohmann-Dumesnil, A. M., M. E., M. D., Ph. D., etc.

Formerly Professor of Dermatology and Syphilology in the St. Louis College for

Medical Practitioners; the St. Louis College of Physicians and Surgeons;

the Marion-Sims College of Medicine; Member of the St. Louis Medical

Society, of the Missouri State Medical Association, of the

American Medical Association, of the Ist, 2d, 3d, 4th, 5th

and 6th International Dermatological

Congress, etc.

\section{THIRD EDITION}

THOROUGHLY REVISED AND ENLARGED

150 ORIGINAL ILLUSTRATIONS

600 PAGES. PRICE: CLOTH, \$4.00. MOROCCO, $\$ 5.50$

\section{PREFACE}

This book is not a treatise. The intention has been to make of it a practical guide to the easy recognition of skin diseases, as well as to their successful treatment. The remedies which have been recommended are such as may be found in cvery practician's armamentarium medicinorum. No attempt has been made to write an elaborate work, but rather to furnisl, in a clear, concise manner, just that information most desired by medical students and general practitioners.

\section{TABLE OF CONTENTS}
Chapter
Chapter
Chapter
I. The Skin.
Chapter
VIII. Prognosis.
II. Anatomy.
III. Physiology.
Chapter
Chapter
IX. Symptomatology.
IV. Diagnosis.
X. Classifications.
Chapter
Chapter
V. Etiology.
Chapter
VI. Pathology.
Chapter
Chapter
XI. Diet in Skin Diseases.
Chapter
XII. Food Eruptions.
Chapter

C. V. Mosby MedicaI Book and Publishing Co.

Grand Ave. and Olive Street, St. Louis, Mo. 


\section{Hand Book of Rectal Diseases}

BY

L. J. HIRSCHMAN, M. D.

Professor of Clinical Proctology Detroit College of Medicine and Surgery.

150 Illustrations

400 Pages. Including 2 Colored Plates. $\quad$ Price $\$ 4.00$.

\section{Gonorrhea in Women}

By

\section{Palmer Findley, M. D.}

Professor of Gynecology in the Medical Department of the University of Nebraska, Omaha, Nebraska.

128 Pages. Royal Octavo. Price $\$ 2.00$.

\section{Chronic Constipation}

By

J. A. McMillian, M. D.

Professor of Therapeutics in the Detroit College of Medicine and Surgery, Detroit, Michigan.

257 Pages. Price $\$ 2.00$.

THE

C. V. MOSBY MEDICAL BOCK AND PUBLISHING CO. St. Louis, Mo. 


\section{Vaccine and Serum Theropy}

By

E. H. Schorer, B. S., M. D.

Assistant Professor of Parositology and Hygiene University of Missouri; formerly Assistant Rockefeller Institute for Medical Research, New York City.

150 Pages. Royal Octavo. Illustrated.

Price, $\$ 2.00$.

\section{Emergency Practice and Formulary}

By

T. A. Hopkins, A. M., M. D.

ST. LOUIS, MO.

300 Pages. Limp Binding. Gilt Top

Pocket Size. Price, $\$ 1.00$.

C. V. Mosby Medical Book and Publishing Co. Grand Ave. and Olive Street., St. Louis, Mo. 


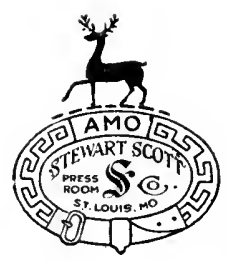




from which it was borrowed. 


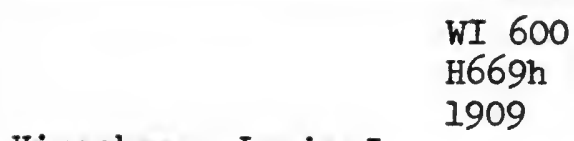

Hirschman, Louis $\mathrm{J}$

Hand book of diseases of the rectu

\section{MEDICAL SCIENCES LIBRARY UNIVERSITY OF CALIFORNIA, IRVINE IRVINE, CALIFORNIA 92664}




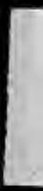

